Prepared for the U.S. Department of Energy

under Contract DE-AC05-76RL01830

\title{
Hanford External Dosimetry Technical Basis Manual PNL-MA-842
}

B. A. Rathbone

January 1, 2010

Pacific Northwest NATIONAL LABORATORY

Proudly Operated by Battelle Since 1965 


\title{
DISCLAIMER
}

This report was prepared as an account of work sponsored by an agency of the United States Government. Neither the United States Government nor any agency thereof, nor Battelle Memorial Institute, nor any of their employees, makes any warranty, express or implied, or assumes any legal liability or responsibility for the accuracy, completeness, or usefulness of any information, apparatus, product, or process disclosed, or represents that its use would not infringe privately owned rights. Reference herein to any specific commercial product, process, or service by trade name, trademark, manufacturer, or otherwise does not necessarily constitute or imply its endorsement, recommendation, or favoring by the United States Government or any agency thereof, or Battelle Memorial Institute. The views and opinions of authors expressed herein do not necessarily state or reflect those of the United States Government or any agency thereof.

\author{
PACIFIC NORTHWEST NATIONAL LABORATORY \\ operated by \\ BATTELLE \\ for the \\ UNITED STATES DEPARTMENT OF ENERGY \\ under Contract DE-AC05-76RL01830
}

Printed in the United States of America
Available to DOE and DOE contractors from the
Office of Scientific and Technical Information,
P.O. Box 62, Oak Ridge, TN 37831-0062;
ph: (865) 576-8401
fax: (865) 576-5728
email: reports@adonis.osti.gov

\author{
Available to the public from the National Technical Information Service, \\ U.S. Department of Commerce, 5285 Port Royal Rd., Springfield, VA 22161 \\ ph: (800) 553-6847$$
\text { fax: (703) 605-6900 }
$$ \\ email: orders@ntis.fedworld.gov \\ online ordering: http://www.ntis.gov/ordering.htm
}


Energy and Environment Directorate

Radiological and Nuclear Science and Technology Division

\section{HANFORD EXTERNAL DOSIMETRY TECHNICAL BASIS MANUAL PNL-MA-842}

Responsible Staff Member:

12

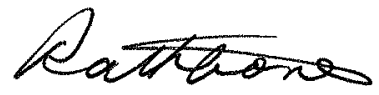

B. A. Rathbone, Technical Manager Hanford External Dosimetry Program Radiation \& Health Technology

Approved for Use by:

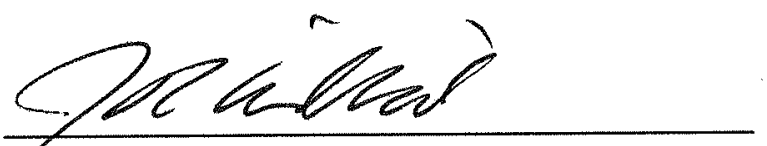

J. R. Hilliard, Program Manager Hanford External Dosimetry Program Radiation \& Health Technology

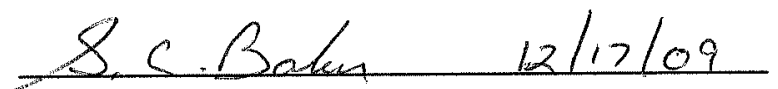

S. C. Baker, Manager

Radiation \& Health Technology

Pacific Northwest National Laboratory Richland, Washington 99352 
This document is available at http://www.pnl.gov/publications/ It is listed as formal report no: PNNL-15750 


\section{Preface}

The Hanford External Dosimetry Program (HEDP) provides support to the U.S. Department of Energy's Richland Operations Office (RL), Office of River Protection (ORP), Pacific Northwest Site Office (PNSO), and DOE contractor radiation protection organizations in determining doses-of-record from external sources of radiation. The Pacific Northwest National Laboratory (PNNL) ${ }^{\text {(a) }}$ administers the HEDP in coordination with Hanford contractor radiation protection organizations to ensure consistent site-wide implementation of external dosimetry practices for Hanford workers and visitors. Coordination of dosimetry practices at Hanford is accomplished through the Hanford Personnel Dosimetry Advisory Committee (HPDAC). Technical services provided by the HEDP include personnel, area, nuclear accident, and environmental dosimetry capabilities that comply with DOE requirements in 10 CFR 835 Occupational Radiation Protection (DOE 2007a), DOE/EH-0027 Department of Energy Standard for the Performance Testing of Personnel Dosimetry Systems (DOE 1986a) and DOE/EH-0026 Handbook for the Department of Energy Accreditation Program for Personnel Dosimetry Systems (DOE 1986b) as well as DOE guidance in DOE G 441.1-1C Radiation Protection Programs Guide (DOE 2008a).

The primary purposes of this Hanford External Dosimetry Technical Basis Manual are to document the design and implementation of the external dosimetry system used at Hanford, and to document the rationale for the measurement methods used. This manual includes documentation of the technical basis for the dosimeter design, processing protocols, dose calculation methodology, and recommended dosimeter use in the field, in a manner intended to demonstrate compliance with 10 CFR 835 and to ensure the defensibility of the doses of record. Secondary purposes of this manual are to provide general information on dosimeter response characteristics and guidance on the proper use and limitations of Hanford dosimeters that will be of use to Hanford radiation protection organizations.

The primary users of this manual are DOE and DOE contractors at Hanford using the dosimetry services of HEDP. Development and maintenance of this manual is funded directly by DOE and DOE contractors. Its contents have been reviewed and approved by DOE and DOE contractors at Hanford through the HPDAC which is chartered and chaired by DOE. This manual supports the Radiation Protection Programs of Hanford contractors.

RL, ORP, PNSO and DOE contractors overseen by these DOE offices will be implementing new requirements in the June 8, 2007 Amendment to 10 CFR 835 Occupational Radiation Protection (DOE 2007a) beginning January 1, 2010. This revision of PNL-MA-842 is consistent with the new requirements and intended for use by these entities on or after January 1, 2010.

(a) Pacific Northwest National Laboratory is operated by Battelle Memorial Institute for the U.S. Department of Energy under Contract DE-AC05-76RLO 1830. 
THIS PAGE INTENTIONALLY LEFT BLANK 


\section{Glossary}

absorbed dose, $D$

accident dosimetry

accreditation

air kerma-to-dose-equivalent conversion factors ( $\mathrm{C}_{\mathrm{k}}$ factors)
(1) The absorbed dose, $D$, is the quotient of $d \bar{\varepsilon}$ by $d m$, where $d \bar{\varepsilon}$ is the mean energy imparted by ionizing radiation to matter of mass $d m$, thus

$$
D=\frac{d \bar{\varepsilon}}{d m}
$$

Unit: $\mathrm{J} \mathrm{kg}^{-1}$

The special name for the unit of absorbed dose is gray (Gy).

This ICRU definition of the absorbed dose, $D$, as a point function, allows the specification of the spatial variations of $D$ as well as the distribution of the absorbed dose in linear energy transfer at the point of interest. $^{\text {(a) }}$

(2) Absorbed dose (D) means the average energy imparted by ionizing radiation to the matter in a volume element per unit mass of irradiated material. The absorbed dose is expressed in units of rad (or gray) (1 rad $=0.01$ gray). ${ }^{\text {(b) }}$

The special unit of absorbed dose is the $\mathrm{rad}$, where $1 \mathrm{rad}=1 \mathrm{erg}$ per gram. One Gy $=100$ rad. The material of interest for absorbed dose (as used in this manual) is typically soft tissue or a phantom approximating soft tissue in composition, but may also include detector materials such as air, methane, silicon, or TLD chip.

Determination of high levels of deep absorbed dose resulting from uncontrolled conditions.

The DOE process of granting accreditation based on onsite assessment against the DOELAP handbook (DOE 1986a) and dosimeter performance testing against the DOELAP standard (DOE 1986b). Accreditation must be updated every two years.

The numerical quantity that relates the air kerma to the dose equivalent at a specified depth in a phantom of specified geometry and composition. Factors are a function of the photon energy and angular distribution.

(a) This is the definition given by the International Commission on Radiation Units and Measurements in ICRU Report 51 Quantities and Units in Radiation Protection Dosimetry (ICRU 1993). It is also the definition used in ANSI/HPS N13.11-2009 American National Standard for Dosimetry - Personnel Dosimetry Performance - Criteria for Testing (HPS 2009).

(b) This is the definition given by DOE in 10 CFR 835 (DOE 2007a). The DOE definition might better be characterized as "average absorbed dose" in the volume element or tissue of interest. In this manual, the term "absorbed dose" may used in a manner consistent with either the DOE or the ICRU definition. 
ALARA

albedo effect

alpha radiation

ambient dose equivalent, $H^{*}(d)$

\section{angular response}

angular dependence

background

\section{beta particle}

beta radiation

bias, $\mathrm{B}$
ALARA is an acronym for "As Low As is Reasonably Achievable," which is the approach to radiation protection to manage and control exposures (both individual and collective) to the work force and to the general public to as low as is reasonable, taking into account social, technical, economic, practical, and public policy considerations.

ALARA is not a dose limit but a process which has the objective of attaining doses as far below the applicable limits of this part as is reasonably achievable.

As used in this document, the neutron dosimeter response caused by the moderating and backscattering properties of a phantom or the human thorax for neutron radiation.

Alpha particles are defined as a helium nucleus with a plus-2 positive charge.

Denoted as $\boldsymbol{H}^{*}(\boldsymbol{d})$ at a point in a radiation field is the dose equivalent that would be produced by the corresponding expanded and aligned field in the ICRU sphere at a depth, $\boldsymbol{d}$, on the radius opposing the direction of the aligned field. The SI unit of ambient dose equivalent is joule per kilogram $\left(\mathrm{J} \mathrm{kg}^{-1}\right)$ and its special name is sievert $(\mathrm{Sv})$. The special unit of ambient dose equivalent is rem. One sievert $=100 \mathrm{rem}$. Ambient dose equivalent is an operational quantity.

The response of a dosimeter or instrument as a function of the angle of incidence of radiation (where normal incidence is designated as zero degrees). The data may be normalized to the response at zero degrees.

Angular dependence of response (same meaning as angular response).

In the 10 CFR 835 definition, background means radiation from (i) naturally occurring radioactive materials which have not been technologically enhanced; (ii) cosmic sources; (iii) global fallout as it exists in the environment (such as from the testing of nuclear explosive devices); (iv) radon and its progeny in concentrations or levels existing in buildings or the environment which have not been elevated as a result of current or prior activities; and (v) consumer products containing nominal amounts of radioactive material, or producing nominal amounts of radiation.

An electron or positron emitted from a nucleus during beta decay.

Radiation consisting of beta particles.

The average of the performance quotients, $\mathrm{P}_{\mathrm{i}}$ for $\mathrm{n}$ dosimeters, for a specified radiation category and test quantity

$$
\mathrm{B} \equiv \overline{\mathrm{P}}=\frac{\sum_{\mathrm{i}=1}^{\mathrm{n}} \mathrm{P}_{\mathrm{i}}}{n}
$$


calibration

chip

committed effective dose, $\mathrm{E}_{50}$

committed equivalent dose, $\mathrm{H}_{\mathrm{T}, 50}$

conventional true value

criticality

cumulative total effective dose

declared pregnant worker

deep absorbed dose, $D(10)$
Calibration means to adjust and/or determine either:

(1) The response or reading of an instrument relative to a standard (e.g., primary, secondary, or tertiary) or to a series of conventionally true values; or

(2) The strength of a radiation source relative to a standard (e.g., primary, secondary, or tertiary) or conventionally true value.

A TLD phosphor in solid form that allows reproducible readout. Typical chip dimensions for the Harshaw dosimetry system are approximately $0.32 \mathrm{~cm} \times 0.32 \mathrm{~cm}$ with thicknesses ranging between 0.15 and $0.89 \mathrm{~mm}$.

The sum of the committed equivalent doses to various tissues or organs in the body $\left(\mathrm{H}_{\mathrm{T}, 50}\right)$, each multiplied by the appropriate tissue weighting factor $\left(\mathrm{w}_{\mathrm{T}}\right)$--that is, $\mathrm{E}_{50}=\Sigma \mathrm{w}_{\mathrm{T}} \mathrm{H}_{\mathrm{T}, 50}+\mathrm{w}_{\text {Remainder }} \mathrm{H}_{\text {Remainder,50. Where }}$ $\mathrm{W}_{\text {Remainder }}$ is the tissue weighting factor assigned to the remainder organs and tissues and $\mathrm{H}_{\text {Remainder,50 }}$ is the committed equivalent dose to the remainder organs and tissues. Committed effective dose is expressed in units of rems (or Sv)

The equivalent dose calculated to be received by a tissue or organ over a 50 -year period after the intake of a radionuclide into the body. It does not include contributions from radiation sources external to the body. Committed equivalent dose is expressed in units of rems (or Sv).

The best estimate of the value of the quantity to be measured, determined by a primary or secondary standard or by a reference instrument that has been calibrated against a primary or secondary standard.

In the context of this document, an unplanned situation in which fissionable material sustains a chain reaction.

The sum of all total effective dose values recorded for an individual plus, for occupational exposures received before January 1, 2010, the cumulative total effective dose equivalent (as defined in the November 4, 1998 amendment to10 CFR 835) values recorded for an individual, where available, for each year occupational dose was received, beginning January 1, 1989.

A woman who has voluntarily declared to her employer, in writing, her pregnancy for the purpose of being subject to the occupational dose limits to the embryo/fetus as provided in 10 CFR 835.206. This declaration may be revoked, in writing, at any time by the declared pregnant worker.

The absorbed dose at a depth of $10 \mathrm{~mm}$ in ICRU tissue. The quantity used for performance testing of dosimeters at accident dose levels. 
deep dose

deep dose equivalent

deterministic effects

directional dose equivalent

\section{DOELAP}

dose

\section{dose algorithm}

dose conversion coefficient
Same as deep dose equivalent

As used in this manual, the terms deep dose and deep dose equivalent (DDE) represent the quantity referred to by the ICRU as "personal dose equivalent" at a depth of $10 \mathrm{~mm}\left(\mathrm{H}_{\mathrm{p}}(10)\right)$. This operational quantity is typically measured for specific radiation types by dosimeters placed on the front torso of the body and is used as an estimate of the effective dose, assuming the body was irradiated in a uniform radiation field. Hanford dosimeters measure and report the beta-photon component $\mathrm{H}_{\mathrm{p}}(10)_{\gamma}$ and neutron component $\mathrm{H}_{\mathrm{p}}(10)_{\eta}$ of deep personal dose equivalent separately. Beta particles contribute a negligible amount to the deep dose equivalent received at Hanford. Any contribution to deep dose equivalent from beta radiation is measured and included in the dosimeter's reported $\mathrm{H}_{\mathrm{p}}(10)_{\gamma}$ result.

Effects due to radiation exposure for which the severity varies with the dose and for which a threshold normally exists (e.g., radiation-induced opacities within the lens of the eye).

Denoted as $\boldsymbol{H}^{\prime}(\boldsymbol{d}, \Omega)$, directional dose equivalent is the dose equivalent at a point in a radiation field that would be produced by the corresponding expanded field in the ICRU sphere at depth, $\mathbf{d}$, on a radius in a specified direction, $\boldsymbol{\Omega}$. The SI unit of directional dose equivalent is joule per kilogram $\left(\mathrm{J} \mathrm{kg}^{-1}\right)$ and its special name is sievert $(\mathrm{Sv})$. The special unit for directional dose equivalent is rem. One sievert $=100 \mathrm{rem}$. Directional dose equivalent is an operational quantity.

Department of Energy Laboratory Accreditation Program administered by the U.S. Department of Energy (DOE 1986a, b).

A general term for any of the following: absorbed dose, dose equivalent, personal dose equivalent, equivalent dose, committed equivalent dose, effective dose, committed effective dose, total effective dose. (a)

A logic flow path or decision tree procedure for calculating dose equivalent from the readings of individual TLD elements in a dosimeter. Sometimes referred to as just "algorithm".

(or dose conversion factor) Dose conversion coefficients are factors for converting readily measurable physical quantities (such as exposure in air, absorbed dose in tissue, kerma in air or tissue, or particle fluence), to operational quantities (such as personal dose equivalent, ambient dose equivalent or directional dose equivalent) or protection quantities (such as equivalent dose or effective dose). These conversion factors generally depend on both the angular and energy distribution of the radiation field (i.e. fluence). They are based on computer calculations

(a) This definition is necessarily broader than the one given in 10 CFR 835 because the primary subject of this manual is measurement of personal dose equivalent. DOE has eliminated all use of the phrase "dose equivalent" from the rulemaking including the general definition of "dose". 
dose equivalent, $H$

dosimeter

dosimeter card

dosimeter holder

dosimetry system

effective dose, $E$ of the operational or protection quantity in the appropriate phantom. For operational quantities, a slab or spherical phantom composed of ICRU tissue is generally used. For protection quantities anthropomorphic phantoms are generally used. Tables of conversion coefficients for monoenergetic radiations are available in ICRU Report 57, Conversion Coefficients for use in Radiological Protection Against External Radiation (ICRU 1998). HPS has prepared a consensus standard for testing personnel dosimeters (HPS N13.11 2009) that uses conversion coefficients for the specific photon, beta and neutron energy spectra, irradiation geometries and phantoms used for dosimeter performance testing that have been determined based on the ICRU 57 monoenergetic conversion factors. DOELAP uses similar spectrum conversion factors in their own dosimeter performance test standard (DOE 1986a) and plans to adopt the HPS standard in the near future.

Denoted as $\boldsymbol{H}$, is the product of Q and $\boldsymbol{D}$ at a point in tissue, where $\boldsymbol{D}$ is the absorbed dose and Q is the quality factor at that point, thus $\boldsymbol{H}=\mathrm{Q} \boldsymbol{D}$. The SI unit of dose equivalent is joule per kilogram $\left(\mathrm{J} \mathrm{kg}^{-1}\right)$ and its special name is sievert (Sv). The special unit of dose equivalent is the rem. One sievert $=100 \mathrm{rem}$. When $\boldsymbol{D}$ is expressed in $\mathrm{rad}, \boldsymbol{H}$ is expressed in rem. When $\boldsymbol{D}$ is expressed in gray, $\boldsymbol{H}$ is expressed in sievert (Sv).

The term dosimeter as used in this manual refers to a device used to assess external radiation exposure or dose to individuals or the environment. HEDP dosimeters are passive devices designed and calibrated to measure either exposure in air, absorbed dose, or personal dose equivalent $\mathrm{H}_{\mathrm{p}}(\mathrm{d})$. The HEDP uses thermoluminescent dosimeters (TLDs) to assess dose to personnel and the environment. A combination of TLDs and activation foils and pellets are used in nuclear accident dosimeters to assess absorbed dose in personnel in the event of a criticality. When used to refer to HEDP personnel, area and environmental dosimeters, the term "dosimeter" generally refers to the complete assembly consisting of a dosimeter card and dosimeter holder.

An aluminum card containing one or more radiation responsive phosphors.

A plastic holder used to contain the dosimeter card. The holder typically has one or more metallic filters used to modify the response of the phosphor to radiation.

A system used to assess dose equivalent from external radiation. This system includes the selection, placement, and processing of the dosimeters; interpretation and recording of results; and the means by which the quality of results is assured.

Denoted as $\boldsymbol{E}$, is the summation of the equivalent doses in specified tissues or organs, each multiplied by the appropriate tissue weighting factor. It is given by the expression 


$$
E=\sum_{T} w_{T} \bullet H_{T}
$$

where $H_{\mathrm{T}}$ is the equivalent dose in tissue or organ, $\mathrm{T}$ and $w_{\mathrm{T}}$ is the appropriate tissue weighting factor for the specified tissue or organ, (see definition for $w_{\mathrm{T}}$ ). For compliance with 10 CFR 835, the tissue weighting factors given in 835.2(b) must be used. For dose from uniform external exposures, deep personal dose equivalent $\mathrm{H}_{\mathrm{p}}(10)$ may be used for effective dose $E$.

The SI unit of effective dose is joule per kilogram (J kg-1) and its special name is sievert (Sv). The special unit of effective dose is rem. One sievert $=100 \mathrm{rem}$. Effective dose is a protection quantity.

NOTE: Effective dose includes the dose from radiation sources internal and/or external to the body. However, as defined here (adapted from ICRP 60 and 10 CFR 835), the contribution from internal sources would not be 50 year commitments (e.g. $\mathrm{E}_{50}$ ), but rather the internal dose received over the specified time period (e.g. calendar year). 10 CFR 835 does not use effective dose in this manner when specifying any dose limit, or monitoring or reporting requirement. When DOE refers to the effective dose from both internal and external sources, the term "total effective dose" (TED) is used which is the sum of E from external sources and $\mathrm{E}_{50}$. When the term effective dose is used (without the word committed), it is generally qualified as effective dose from external sources. Therefore, when used in this manual, which is concerned primarily with external dose, the symbol $E$ will denote effective dose resulting from external sources, unless specified otherwise. The symbol $E_{\gamma}$ will denote the component resulting from photon radiation and the symbol $E_{\eta}$ will denote the component resulting from neutron radiation.

\section{element}

\section{element correction coefficient (ECC)}

A dosimeter detector that provides a single readout value. TLD elements may be solid chips or powdered phosphors bonded on substrates suitable for heating such as Kapton ${ }^{\mathrm{TM}}$. The terms "chip" and "element" are often used interchangeably in the context of the Harshaw dosimeters and TLD system described in this manual.

Correction factors used to normalize the sensitivity of an individual dosimeter element, to the mean sensitivity of a reference population (calibration set) exposed to the same source. The ECC is determined as follows:

$$
\mathrm{ECC}_{\mathrm{ij}}=\frac{\mathrm{RCF}_{\mathrm{i}}}{\mathrm{Q}_{\mathrm{ij}}} \mathrm{X}
$$

where

$\mathrm{ECC}_{\mathrm{ij}}=$ element correction coefficient for chip $\mathrm{i}$ on card $\mathrm{j}$ 
equivalent dose, $H_{\mathrm{T}, \mathrm{R}}$ and $H_{\mathrm{T}}$

equivalent dose to the extremity

\section{equivalent dose to the lens of the eye}

$\mathrm{RCF}_{\mathrm{i}}=$ reader calibration factor for ith photomultiplier tube.

$\mathrm{Q}_{\mathrm{ij}} \quad=$ reported charge from chip $\mathrm{i}$ on card $\mathrm{j}$

$\mathrm{X}={ }^{60}$ Co exposure value.

$\boldsymbol{H}_{\mathrm{T}, \mathrm{R}}$ is the absorbed dose in an organ or tissue due to a given radiation, multiplied by the radiation weighting factor. Thus $\boldsymbol{H}_{\mathrm{T}, \mathrm{R}}=\mathrm{W}_{\mathrm{R}} \cdot \boldsymbol{D}_{\mathrm{T}, \mathrm{R}}$ where $\boldsymbol{D}_{\mathrm{T}, \mathrm{R}}$ is the absorbed dose averaged over the tissue or organ, T, due to radiation $R$, and $\mathrm{w}_{\mathrm{R}}$ is the radiation weighting factor for radiation $\mathrm{R}$. When the radiation field is composed of radiations with different values of $\mathrm{w}_{\mathrm{R}}$, the total equivalent dose $\boldsymbol{H}_{\mathrm{T}}$ is calculated as the summation of $\boldsymbol{H}_{\mathrm{T}, \mathrm{R}}$ over all radiations R, i.e.,

$$
H_{T}=\sum_{R} H_{T, R}=\sum_{R} w_{R} \bullet D_{T, R}
$$

The SI unit of equivalent dose is joule per kilogram $\left(\mathrm{J} \mathrm{kg}^{-1}\right)$ and its special name is sievert $(\mathrm{Sv})$. The special unit of equivalent dose is rem. One sievert $=100$ rem. Equivalent dose is a protection quantity.

For dose from external radiation fields, the DOE protection quantity "equivalent dose to the extremity" will be estimated using the ICRU operational quantity $\mathrm{H}_{\mathrm{p}}(0.07)$, measured at an appropriate location on the extremity.

NOTE: The term "equivalent dose to the extremity" is used in 10 CFR 835 but not explicitly defined. By inference from the definition of equivalent dose, equivalent dose to an extremity would be $H_{\mathrm{T}}$ where tissue $\mathrm{T}$ is the extremity and $D_{\mathrm{T}}$ is the absorbed dose averaged over that extremity. However the 835.2(b) definition for equivalent dose states the following: "...the equivalent dose to the extremity and skin is assessed at a depth of $0.007 \mathrm{~cm}$ in tissue..." This implies DOE's intended use of the operational quantity $\mathrm{H}_{\mathrm{p}}(0.07)$ which is consistent with the guidance given in DOE G 441.1-1C Radiation Protection Programs Guide (DOE 2008).

For dose from external radiation fields, the DOE protection quantity "equivalent dose to the lens of the eye" will be estimated using the ICRU operational quantity $\mathrm{H}_{\mathrm{p}}(3)$, measured at an appropriate location on the body. For non-uniform radiation fields, the location should be near the lens of the eye.

NOTE: The term "equivalent dose to the lens of the eye" is used in 10 CFR 835 but not explicitly defined. By inference from the definition of equivalent dose, equivalent dose to the lens of the eye would be $H_{\mathrm{T}}$ where tissue $\mathrm{T}$ is the lens of the eye and $D_{\mathrm{T}}$ is the absorbed dose averaged over the lens. However the 835.2(b) definition for equivalent dose states the following: "...the equivalent dose to the lens of the eye is assessed at a depth of $0.3 \mathrm{~cm}$ in tissue..." This implies DOE's intended use of the operational quantity $\mathrm{H}_{\mathrm{p}}(3)$ which is consistent with the guidance given in DOE G 441.1-1C Radiation Protection Programs Guide (DOE 2008). 
equivalent dose to the skin

exposure

exposure-to-dose-equivalent conversion factors ( $\mathrm{C}_{\mathrm{x}}$ factors)
For dose from external radiation fields, the DOE protection quantity "equivalent dose to the skin" will be estimated using the ICRU operational quantity $\mathrm{H}_{\mathrm{p}}(0.07)$, personal dose equivalent at a depth of $0.07 \mathrm{~mm}$ measured at an appropriate location on the body. For nonuniform radiation fields, equivalent dose to the skin must be assessed according to 10 CFR 835.205.

NOTE: The term "equivalent dose to the skin" is used in 10 CFR 835 but not explicitly defined. By inference from the definition of equivalent dose, equivalent dose to the skin would be $H_{\mathrm{T}}$ where tissue $\mathrm{T}$ is the skin of the whole body and $D_{\mathrm{T}}$ is the absorbed dose averaged over the skin mass. However the 835.2(b) definition for equivalent dose states the following: "...the equivalent dose to the extremity and skin is assessed at a depth of $0.007 \mathrm{~cm}$ in tissue..." This implies DOE's intended use of the operational quantity $\mathrm{H}_{\mathrm{p}}(0.07)$ which is consistent with the guidance given in DOE G 441.1-1C Radiation Protection Programs Guide (DOE 2008).

equivalent dose to the whole body For uniform external radiation fields, the DOE protection quantity "equivalent dose to the whole body" will be estimated using the ICRU operational quantity $\mathrm{H}_{\mathrm{p}}(10)$, measured at an appropriate location on the body. For non-uniform external radiation fields, the assessed effective dose from multiple dosimeter data will be used for equivalent dose to the whole body.

NOTE: This term is used in 10 CFR 835 but not explicitly defined. ICRP does not identify the "whole body" as a tissue or organ. By inference from the definition of equivalent dose, equivalent dose to the whole body would be $H_{\mathrm{T}}$ where tissue $\mathrm{T}$ is the whole body and $D_{\mathrm{T}}$ is the absorbed dose averaged over the mass of the whole body. However the 835.2(b) definition for equivalent dose states the following: "For external dose, the equivalent dose to the whole body is assessed at a depth of $1 \mathrm{~cm}$ in tissue..." This implies DOE's intended use of the operational quantity $\mathrm{H}_{\mathrm{p}}(10)$, which is consistent with the guidance given in DOE G 441.1-1C Radiation Protection Programs Guide (DOE 2008).

The term "exposure" technically refers to a physical quantity traditionally used to define the strength of a photon radiation field in terms of the resulting ionization in air, in units of roentgen (R) or coulombs per kilogram. For the purpose of specifying photon radiation fields to demonstrate traceability to national standards labs (e.g. NIST, PTB, NPL), the quantity exposure in air has been replaced with kerma in air. The term "exposure" is often used in a generic sense to describe the process of exposing a person or dosimeter to radiation.

The numerical quantity that relates the exposure in air to the dose equivalent at a specified depth in a phantom of specified geometry and composition. Factors are a function of the photon energy, and angular distribution. 
external dose or exposure

external dosimetry

extremity

eye dose

eye dose equivalent

\section{facility specific calibration factor}

\section{fissile materials}

fluence, $\Phi$

\section{free field dose equivalent}

\author{
free field fluence, $\Phi$
}

That portion of the dose received from radiation sources outside the body (i.e. "external sources").

Theory and application of the principles and techniques involved in the measurement and recording of radiation absorbed dose, dose equivalent, equivalent dose, and effective dose in personnel from external sources of radiation. The objective of external dosimetry is the assessment of personnel exposure to external radiation in terms of the operational and protection dosimetric quantities used to measure and limit personnel dose.

Hands and arms below the elbow or feet and legs below the knee.

Same as eye dose equivalent.

As used in this manual, the terms eye dose equivalent and eye dose are synonymous with the ICRU operational quantity $\mathrm{H}_{\mathrm{p}}(3)$, the personal dose equivalent at a depth of $3 \mathrm{~mm}$ in the human body, or in a phantom of tissue equivalent material.

The dosimeter calibration factor applicable to a particular occupational environment. These calibration factors are determined by comparing reference instrument measurements with dosimeter response measurements. Both measurements are performed in the workplace.

Uranium-233, uranium-235, plutonium-239, plutonium-241, americium$242 \mathrm{~m}$, californium-249, californium-251, curium-243, curium-245, and curium-247, or any material containing any of the foregoing, with the following exceptions: materials containing natural or depleted uranium are not considered to be fissile materials.

The quotient of $\mathrm{d} N$ by $\mathrm{d} a$ where $\mathrm{d} N$ is the number of particles incident on a sphere of cross sectional area $\mathrm{d} a$

$$
\Phi=\frac{d N}{d a}
$$

The delivered dose equivalent at a point in space, based on the free field fluence at that point and the fluence to dose equivalent conversion factor used to convert fluence to dose equivalent in a phantom, for the given neutron spectrum. Used to define reference radiation fields for the purpose of dosimeter calibration.

The fluence at a point in space that would occur if the irradiation were performed in the absence of air, walls, phantoms, or other scattering materials - usually applied to neutron radiation. Used to define reference radiation fields for the purpose of dosimeter calibration. 
general employee

$\mathbf{H}_{\mathrm{p}} \mathbf{( 1 0 )}$

$\mathbf{H}_{\mathrm{p}}(3)$

$\mathbf{H}_{\mathrm{p}}(\mathbf{0 . 0 7})$

in-air exposure

individual

internal dosimetry

ionizing radiation

irradiation category

kerma, $\mathbf{K}$
An individual who is either a DOE or DOE contractor employee: an employee of a subcontractor to a DOE contractor; or an individual who performs work for or in conjunction with DOE or utilizes DOE facilities.

Deep personal dose equivalent (i.e. personal dose equivalent specified at a depth of $10 \mathrm{~mm})$. As used in this manual, $\mathrm{H}_{\mathrm{p}}(10)$ is synonymous with the terms deep dose equivalent and deep dose. Hanford dosimeters measure and report the beta-photon and neutron components of $\mathrm{H}_{\mathrm{p}}(10)$ separately. In this manual, the beta-photon component is denoted by $\mathrm{H}_{\mathrm{p}}(10)_{\gamma}$ and the neutron component is denoted by $\mathrm{H}_{\mathrm{p}}(10)_{\eta}$. The total deep personal dose equivalent, $\mathrm{H}_{\mathrm{p}}(10)=\mathrm{H}_{\mathrm{p}}(10)_{\gamma}+\mathrm{H}_{\mathrm{p}}(10)_{\eta}$. At Hanford, beta particles contribute a negligible amount to $\mathrm{H}_{\mathrm{p}}(10)$.

Eye personal dose equivalent (i.e. personal dose equivalent specified at a depth of $3 \mathrm{~mm}$ ). As used in this manual, $\mathrm{H}_{\mathrm{p}}(3)$ is synonymous with the terms eye dose equivalent and eye dose. The value of $\mathrm{H}_{\mathrm{p}}(3)$ reported by Hanford dosimeters includes only the dose from beta particles and photons. It does not include the dose from neutrons. For the purpose of estimating the protection quantity equivalent dose to the lens of the eye, $\mathrm{H}_{\mathrm{p}}(10)_{\eta}$ is added to $\mathrm{H}_{\mathrm{p}}(3)$. As used in this manual, the symbol $\mathrm{H}_{\mathrm{p}}(3)$ generally refers to the beta-photon component of $\mathrm{H}_{\mathrm{p}}(3)$.

Shallow personal dose equivalent (i.e. personal dose equivalent specified at a depth of $0.07 \mathrm{~mm})$. As used in this manual, $\mathrm{H}_{\mathrm{p}}(0.07)$ is synonymous with the terms shallow dose equivalent and shallow dose. The value of $\mathrm{H}_{\mathrm{p}}(0.07)$ reported by Hanford dosimeters includes only the dose from beta particles and photons. It does not include the dose from neutrons. For the purpose of estimating the protection quantity equivalent dose to the skin, $\mathrm{H}_{\mathrm{p}}(10)_{\eta}$ is added to $\mathrm{H}_{\mathrm{p}}(0.07)$. As used in this manual, the symbol $\mathrm{H}_{\mathrm{p}}(0.07)$ generally refers to the beta-photon component of $\mathrm{H}_{\mathrm{p}}(0.07)$.

As used in this document, exposure of a dosimeter without any phantom or other backscatter material nearby.

Any human being.

Theory and application of the principles and techniques involved in the measurement and recording of radiation dose from sources of radiation internal to the human body.

Any radiation capable of displacing electrons from atoms or molecules, thereby producing ions.

DOELAP performance testing radiation types and energies (or mixtures) for which performance criteria are given.

the quotient of $\mathrm{d} E_{\text {tr }}$ by $\mathrm{d} m$, where $\mathrm{d} E_{\text {tr }}$ is the sum of the initial kinetic energies of all the charged ionizing particles liberated by uncharged ionizing particles in a volume element of mass $\mathrm{d} m$. 


$$
K=\frac{d E_{t r}}{d m}
$$

The unit of kerma is joule per kilogram $\left(\mathrm{J} \mathrm{kg}^{-1}\right)$ and its special name is gray (Gy). The special unit of kerma is rad. One gray $=100 \mathrm{rad}$.

$\mathbf{L}_{\mathbf{C}}$

$\mathbf{L}_{\mathbf{D}}$

\section{lens of the eye}

linear energy transfer, $L$

lower limit of detection (LLD)

may

mil

minor

monitoring

neutron activation critical level (after Roberson and Carlson 1992), [also referred to as "detection limit" (Currie 1968)]. The signal level above which, at a predetermined confidence level (typically 95\%), a signal may be considered to be outside the expected range of fluctuation of the background signal.

detection level (after Roberson and Carlson 1992), [also referred to as "decision limit" (Currie 1968)]. The delivered quantity (e.g. exposure or dose) for which there is a predetermined confidence level (typically 95\%) that the associated signal will be detected (i.e. greater than the critical level $\mathrm{L}_{\mathrm{C}}$ ) and the quantity be reported as a qualitative positive result. $L_{D}$ is commonly referred to as the lower limit of detection (LLD).

An tissue of concern from a dosimetric and regulatory point of view. The lens of the eye lies at a depth of approximately $3 \mathrm{~mm}$.

Linear energy transfer (LET) is the quotient of $\mathrm{d} E$ by $\mathrm{d} l$, where $\mathrm{d} E$ is the mean energy lost by the particle, owing to collisions with electrons, in traversing a distance $\mathrm{d} l$. (also referred to as linear collision stopping power of a material for charged particles). The mathematical symbol commonly used for linear energy transfer is $\mathrm{L}$

$$
L=\frac{d E}{d l}
$$

Same as $\mathbf{L}_{\mathbf{D}}$.

Denotes permission, rather than recommendation or requirement.

Unit used to specify thickness of materials; equals 0.001 inch or 0.025 $\mathrm{mm}$.

An individual less than 18 years of age.

The measurement of radiation levels, airborne radioactivity concentrations, radioactive contamination levels, quantities of radioactive material, or individual doses and the use of the results of these measurements to evaluate radiological hazards or potential and actual doses resulting from exposures to ionizing radiation.

The process in which atomic nuclei become radioactive by absorption of neutrons. 


\section{neutron deep dose}

neutron deep dose equivalent

\section{non-uniform fields (irradiation)}

occupational dose

on-phantom

operational quantities

performance testing
Same as neutron deep dose equivalent.

As used in this manual, this term is synonymous with the personal dose equivalent at a depth of $10 \mathrm{~mm}$ in the human body or in a phantom of tissue equivalent material, resulting from incident neutrons, as denoted by the symbol $\mathrm{H}_{\mathrm{p}}(10)_{\eta}$. The quantity includes dose equivalent from photons produced by capture reactions within the body.

The condition when a portion of the body is expected to receive a radiation dose equivalent that varies by more than $50 \%$ from the dose equivalent expected at a reference location (e.g., the anterior torso).

An individual's ionizing radiation dose (external and internal) as a result of that individual's work assignment. Occupational dose does not include doses received as a medical patient or doses resulting from background radiation or participation as a subject in medical research programs.

As used in this document, exposure of dosimeters affixed to a phantom to simulate the dosimeter response while the dosimeter is being worn by a person.

Operational quantities are measurable quantities specified by the ICRU that may be used to demonstrate compliance with regulatory dose limits expressed as protection quantities. Operational quantities are intended to provide a conservative estimate of their related protection quantities. Examples of operational quantities are personal dose equivalent $\mathrm{H}_{\mathrm{p}}(\mathrm{d})$, ambient dose equivalent $H^{*}(d)$ and directional dose equivalent $H^{\prime}(d, \Omega)$.

Procedure with the following sequence:

1. Submission of dosimeters from a processor's current stock to a testing laboratory over a period of several months, in numbers sufficient for the specified irradiations in any one test category or subcategory covered by a processor's service.

2. Irradiation of the dosimeters by personnel of the testing laboratory using the type(s) of radiation specified for this test category or subcategory.

3. Evaluation by the processor of the response of the returned dosimeters in terms of shallow and deep dose equivalent for tests of protection monitoring, or in terms of deep absorbed dose for tests of accident monitoring.

4. Submission of these evaluations to the testing laboratory.

5. Analysis of the submitted evaluations by the testing laboratory.

6. Reporting of the results of this analysis (also referred to as "test 
performance testing category

\section{performance testing laboratory (PTL)}

performance quotient, $\mathbf{P}_{\mathrm{i}}$

personal dose equivalent, $H_{p}(d)$

person

\section{phantom}

results") to the processor.

Each type of radiation (or of radiation mixtures) and range of irradiation level for which separate tests are performed.

The DOELAP dosimeter performance testing laboratory.

For tests of protection dosimetry, the performance quotient for the $i^{\text {th }}$ dosimeter is defined as:

$$
P_{i} \equiv\left[H_{R, i}-H_{G, i}\right] / H_{G, i}
$$

where $H_{R, i}$ is the dose equivalent reported by the test participant (processor), and $H_{G, i}$ is the given dose equivalent (conventionally true value) assigned by the testing laboratory to the irradiated dosimeter.

For tests of accident dosimetry, the same definition applies, with the absorbed dose, D, replacing the dose equivalent, $H$.

NOTE: In this definition, $\mathrm{H}$ stands for $\mathrm{H}_{\mathrm{p}}(10)$ or $\mathrm{H}_{\mathrm{p}}(0.07)$ and $\mathrm{D}$ stands for $D(10)$. No tests are performed for $\mathrm{H}_{\mathrm{p}}(3), D(3)$ or $D(0.07)$.

Denoted as $\mathrm{H}_{\mathrm{p}}(\mathrm{d})$, is the dose equivalent in soft tissue at an appropriate depth, $d$, (in millimeters) below a specified point on the body. The unit of personal dose equivalent is joule per kilogram $\left(\mathrm{J} \mathrm{kg}^{-1}\right)$ and its special name is sievert. The special unit for personal dose equivalent is rem. One sievert $=100 \mathrm{rem}$. Personal dose equivalent is an operational quantity.

Any individual, corporation, partnership, firm, association, trust, estate, public or private institution, group, Government agency, any State or political subdivision of, or any political entity within a State, any foreign government or nation or other entity, and any legal successor, representative, agent or agency of the foregoing; provided that person does not include DOE or the United States Nuclear Regulatory Commission. [Note: This definition is the same one given in $10 \mathrm{CFR}$ 835.2]

A physical substitute for the human body used when irradiating personnel dosimeters. The purpose of the phantom is to provide similar radiation absorption and scattering properties as human tissue and thus an influence on dosimeter response that is approximately equivalent to that of the human body. Phantoms may be of any size or shape but are generally approximately tissue equivalent for the radiation type being measured. Typical phantoms used for extremity dosimeter calibration and performance testing are the polymethylmethacrylate (PMMA) plastic rod (finger) or pillar (wrist) phantom. Typical phantoms used for calibration and performance testing of whole body dosimeters are the PMMA slab phantom, measuring either $30-b y-30 \mathrm{~cm}$ square by $15-\mathrm{cm}$ deep or 40-x-40-cm by $15-\mathrm{cm}$ deep. Solid or water filled slabs and 


\section{phosphor}

photon radiation

physical quantities

protection quantities

quality factor, $\mathbf{Q}$

quality assurance (QA)

radiation cylinders are frequently used for area dosimeters and for field measurements with personnel dosimeters. Anthropomorphic phantoms with shapes more closely approximating the human body are typically used for dosimetry studies and calculational models..

As used in this report, a material with the characteristic of emitting light following irradiation. Thermoluminescent phosphors emit this light (luminesce) under heating (thermo).

Refers to either $\mathrm{x}$ or gamma rays.

Quantities used to measure the fundamental physical properties of radiation fields and radiation interaction in matter. Examples of physical quantities are: fluence $\Phi$, air kerma $K_{\mathrm{a}}$, tissue absorbed dose $D$, linear energy transfer $L$. Relationships between physical quantities, damage in tissue, and risk of stochastic effects are used to calculate the protection quantities and operational quantities used in radiation protection.

Dosimetric quantities specified in the human body by the ICRP for the purpose of dose limitation. Examples of protection quantities are equivalent dose in a tissue or organ $H_{\mathrm{T}}$ and effective dose $E$ (ICRP 60). Protection quantities are intended to serve as the basis for dose limitation adopted by regulators and other bodies. DOE currently uses effective dose and equivalent dose as the basis for dose limits. Protection quantities can be readily calculated but are generally difficult to measure directly. Operational quantities are more readily measured and are used to demonstrate compliance with dose limits expressed as protection quantities.

The quality factor $(\mathrm{Q})$ is the modifying factor by which absorbed dose (D) is multiplied to obtain the dose equivalent. Q is a function of unrestricted linear energy transfer, $L$, in water. Values of $Q(L)$ as a function of L are given in ICRP Publication 60 (ICRP, 1991) by the following relations:

\begin{tabular}{|l|l|}
\hline $\mathrm{L}<10$ & $\mathrm{Q}(\mathrm{L})=1$ \\
\hline $10 \leq \mathrm{L} \leq 100$ & $\mathrm{Q}(\mathrm{L})=0.32 \mathrm{~L}-2.2$ \\
\hline $\mathrm{L}>100$ & $\mathrm{Q}(\mathrm{L})=300 / \sqrt{\mathrm{L}}$ \\
\hline
\end{tabular}

All planned and periodic actions necessary to provide adequate confidence that an item or a service will satisfy given needs.

Unless otherwise specified, radiation refers to particulate or electromagnetic radiation that is ionizing. Examples of indirectly ionizing radiation significant at Hanford are gamma rays, $\mathrm{x}$-rays, and neutrons. Examples of directly ionizing radiation encountered at Hanford are alpha particles and beta particles. 
Radiation weighting factor $\left(\mathrm{w}_{\mathrm{R}}\right)$ means the modifying factor used to calculate the equivalent dose from the average tissue or organ absorbed dose; the absorbed dose (expressed in rad or gray) is multiplied by the appropriate radiation weighting factor. The radiation weighting factors to be used for determining equivalent dose in rems are as follows:

$$
\text { RADIATION WEIGHTING FACTORS }{ }^{1}, \mathrm{w}_{\mathrm{R}}
$$

\begin{tabular}{|c|c|}
\hline Radiation Type R & $\begin{array}{l}\text { Weighting } \\
\text { Factor } w_{\mathrm{R}}\end{array}$ \\
\hline Photons, electrons and muons, all energies & 1 \\
\hline Neutrons, energy $<10 \mathrm{keV}^{2,3}$ & 5 \\
\hline Neutrons, energy $10 \mathrm{keV}$ to $100 \mathrm{keV}^{2,3}$ & 10 \\
\hline Neutrons, energy $>100 \mathrm{keV}$ to $2 \mathrm{MeV}^{2,3}$ & 20 \\
\hline Neutrons, energy $>2 \mathrm{MeV}$ to $20 \mathrm{MeV}^{2,3}$ & 10 \\
\hline Neutrons, energy $>20 \mathrm{MeV}^{2,3}$ & 5 \\
\hline Protons, other than recoil protons, energy $>2 \mathrm{MeV}$ & 5 \\
\hline Alpha particles, fission fragments, heavy nuclei & 20 \\
\hline \multicolumn{2}{|c|}{$\begin{array}{l}\text { All values relate to the radiation incident on the body or, for internal sources } \\
\text { emitted from the source. } \\
\text { When spectral data are insufficient to identify the energy of the neutrons, a } \\
\text { radiation weighting factor of } 20 \text { shall be used. } \\
\text { When spectral data are sufficient to identify the energy of the neutrons, the } \\
\text { following equation may be used to determine a neutron radiation weighting } \\
\text { factor value: }\end{array}$} \\
\hline
\end{tabular}

$w_{\mathrm{R}}=5+17 \exp \left[\frac{-\left(\ln \left(2 E_{n}\right)\right)^{2}}{6}\right]$

Where $E_{n}$ is the neutron energy in $\mathrm{MeV}$.

\section{radioactivity}

radiological worker

relative biological effectiveness, $\mathbf{R B E}_{\mathbf{M}}$

reporting threshold
Unstable isotopes that release energy in the form of particles and/or electromagnetic radiation by a process of disintegration.

A general employee whose job assignment involves operation of radiation producing devices or working with radioactive materials, or who is likely to be routinely occupationally exposed above 0.1 rem per year total effective dose.

The ratio of the absorbed dose of a reference radiation to the absorbed dose of a given test radiation required to produce the same level of response, all other conditions being kept constant. The subscript $\mathrm{M}$ refers to a stochastic effect.

The calculated dose level below which the dose result will be reported as zero. 
response

roentgen (R)

shall

shallow absorbed dose, $D(0.07)$

shallow dose

shallow dose equivalent

\section{should}

skin

standard deviation, $\mathrm{S}$

stochastic effects
The quotient of the reading of a measuring instrument or dosimeter and the conventional true value of the measured quantity.

A special unit of radiation used to quantify ionization in air from photon radiation. One $\mathrm{R}$ is equivalent to $2.58 \times 10^{-4}$ coulomb $/ \mathrm{kg}$.

Denotes a requirement.

The absorbed dose at a depth of $0.07 \mathrm{~mm}$ in ICRU tissue.

Same as shallow dose equivalent.

As used in this manual, the terms shallow dose and shallow dose equivalent (SDE) are synonymous with the ICRU operational quantity $\mathrm{H}_{\mathrm{p}}(0.07)$, personal dose equivalent at a depth of $0.07 \mathrm{~mm}$. This operational quantity is typically measured by dosimeters placed on the front torso of the body or on the extremities of the body. Hanford dosimeters measure and report only the beta-photon component of $\mathrm{H}_{\mathrm{p}}(0.07)$. As used in this manual, the symbol $\mathrm{H}_{\mathrm{p}}(0.07)$ generally refers to the beta-photon component of shallow dose equivalent. For the purpose of estimating equivalent dose to the skin or any extremity, the reported neutron deep dose equivalent $H_{p}(10)_{\eta}$ is added to $H_{p}(0.07)$.

Denotes a recommendation.

The thickness of the skin varies considerably from one part of the body to another. The basal cell layer of the epidermis is taken to be the skin tissue most at risk. For dose assessment purposes, a depth of $70 \mu \mathrm{m}$ is considered to be the mean depth of the basal cell layer.

The standard deviation of the performance quotient, $\mathrm{P}_{\mathrm{i}}$, is determined as follows:

$$
\mathrm{S} \equiv\left\{\left[\sum_{\mathrm{i}=1}^{\mathrm{n}}\left(\mathrm{P}_{\mathrm{i}}-B\right)^{2}\right] /(\mathrm{n}-1)\right\}^{1 / 2}
$$

where the sum is extended over all $n$ values of $P_{i}$ for a particular test in a given radiation category or subcategory, and for a particular phantom depth (shallow or deep) and

$$
B=(1 / \mathrm{n}) \sum_{\mathrm{i}=1}^{\mathrm{n}} \mathrm{P}_{\mathrm{i}}
$$

Malignant and hereditary diseases for which the probability of an effect occurring, rather than its severity, is regarded as a function of dose without a threshold, for radiation protection purposes. 


\section{thermoluminescent dosimeter (TLD)}

tissue weighting factor, $w_{\mathrm{T}}$
A type of dosimeter that relies on excitation of the crystalline lattice by radiation of certain fluorescent materials which, upon heating, emit light. Various phosphors and chemical activators have led to several common types of thermoluminescent phosphors. In this document, reference is made primarily to lithium fluoride $(\mathrm{LiF})$ and to calcium fluoride $\left(\mathrm{CaF}_{2}\right)$.

A factor by which the equivalent dose to a tissue or organ, $\mathrm{H}_{\mathrm{T}}$ is multiplied in order to account for the relative stochastic detriment resulting from the exposure of different tissues and organs. In other words, $\mathrm{w}_{\mathrm{T}}$ represents the fraction of the overall health risk, resulting from uniform, whole body irradiation, attributable to specific tissue (T). The tissue weighting factors used for determining effective dose for DOE workers are given below.

\begin{tabular}{|l|c|}
\hline Organs or tissues, $\mathrm{T}$ & Tissue weighting factor, $w_{\mathrm{T}}$ \\
\hline Gonads & 0.20 \\
Red bone marrow & 0.12 \\
Colon & 0.12 \\
Lungs & 0.12 \\
Stomach & 0.12 \\
Bladder & 0.05 \\
Breast & 0.05 \\
Liver & 0.05 \\
Esophagus & 0.05 \\
Thyroid & 0.05 \\
Skin & 0.01 \\
Bone surfaces & 0.01 \\
Remainder & 0.05 \\
Whole body $^{2}$ & 1.00 \\
\hline
\end{tabular}

1 "Remainder" means the following additional tissues and organs and their masses, in grams, following parenthetically: adrenals (14), brain (1400), extrathoracic airways (15), small intestine (640), kidneys (310), muscle $(28,000)$, pancreas $(100)$, spleen $(180)$, thymus $(20)$, and uterus $(80)$. The equivalent dose to the remainder tissues $\left(\mathrm{H}_{\text {remainder }}\right)$, is normally calculated as the mass-weighted mean dose to the preceding ten organs and tissues. In those cases in which the most highly irradiated remainder tissue or organ receives the highest equivalent dose of all the organs, a weighting factor of 0.025 (half of remainder) is applied to that tissue or organ and 0.025 (half of remainder) to the mass-weighted equivalent dose in the rest of the remainder tissues and organs to give the remainder equivalent dose.

2 For the case of uniform external irradiation of the whole body, a tissue weighting factor $\left(\mathrm{w}_{\mathrm{T}}\right)$ equal to 1 may be used in determination of the effective dose. 
Total effective dose (TED)

track-etch dosimeter

Whole body
The sum of the effective dose (from external exposures) and the committed effective dose.

A type of dosimeter that relies on the production of tracks in a plastic to measure dose. Radiation damage sites in the CR-39 plastic produce tracks or "pits" when electrochemically etched, which can be seen and counted under a microscope. The formation of these tracks is primarily caused by hydrogen recoil from fast neutrons, but can also be caused by alpha particles, protons, and heavy charged particles.

Whole body means, for the purposes of external exposure, head, trunk (including male gonads), arms above and including the elbow, or legs above and including the knee. 


\section{Acronyms and Abbreviations}

ABS

ACL

AEDE

ALARA

$\mathrm{AMH}$

ANSI

AP

BCF

CCS

CED

CEqD

CFR

CPE

CPM

CV

DDE

DOE

DOE-RL

DOELAP

DRD

DU

ECC

ED

EED

FNAD acrylonitrile-butadiene-styrene

administrative control level

annual effective dose equivalent

as low as reasonably achievable

AdvanceMed Hanford

American National Standards Institute

anterior-posterior (exposure geometry)

beta correction factor

computer control system

committed effective dose

committed equivalent dose

Code of Federal Regulations

charged particle equilibrium

counts per minute

coefficient of variation

deep dose equivalent

U.S. Department of Energy

U.S. Department of Energy - Richland Operations Office

Department of Energy Laboratory Accreditation Program

direct reading dosimeter

depleted uranium

element correction coefficient

effective dose, External Dosimetry

Energy and Environment Directorate

fixed nuclear accident dosimeter 
GDS

GM

HCND

HEDP

HEPA

HIDP

HPDAC

HPRR

HPS

HRCF

HRRP

HSD

ICRP

ICRU

ID

IODR

ISO

ISO

JST

LANL

LAT

LET

LLD

LOI

MMD

MOU
Global Dosimetry Solutions (company name)

Geiger-Müller (counter)

Hanford Combination Neutron Dosimeter

Hanford External Dosimetry Program

High Efficiency Particulate Air (filter)

Hanford Internal Dosimetry Program

Hanford Personnel Dosimetry Advisory Committee

Health Physics Research Reactor

Health Physics Society

Hanford Radiological Control Forum

Hanford Radiation Records Project

Hanford Standard Dosimeter

International Commission on Radiological Protection

International Commission on Radiation Quantities and Units

identification

investigation of dosimeter result

isotropic (exposure geometry)

International Standards Organization

Job Specific Training

Los Alamos National Laboratory

lateral (exposure geometry)

linear energy transfer

lower limit of detection

letter of instruction

minimum measurable dose

memorandum of understanding 
NAD

NCRP

NHC

NIST

NRC

NVLAP

OCR

OJT

ORNL

ORP

PA

PC

PFP

PMMA

PMT

PNAD

PNNL

PNSO

PTB

PTFE

QA

QC

RCF

REMS

REX

R\&HT

RIDS nuclear accident dosimeter

National Council on Radiation Protection and Measurements

Numatec Hanford Corporation

National Institute of Standards and Technology

U.S. Nuclear Regulatory Commission

National Voluntary Laboratory Accreditation Program

optical character reader

on-the-job-training

Oak Ridge National Laboratory

DOE Office of River Protection

posterior-anterior (exposure geometry)

personal computer

Plutonium Finishing Plant

polymethylmethacrylate

photomultiplier tube

personnel nuclear accident dosimeter

Pacific Northwest National Laboratory

Pacific Northwest Site Office (DOE Office of Science)

Physikalisch-Technische Bundesanstalt

polytetrafluorethylene

quality assurance

quality control

reader calibration factor

Radiation Evaluation and Management System (TLD reader system)

Radiological Exposure (System)

Radiation \& Health Technology

records inventory and disposition schedule 
RL

ROT

RPP

RRF

RWP

SDE

SOW

TED

TEPC

TL

TLD

TTP

UPS

UV

VAX

WB

WCH

WHC

YTD
DOE Richland Operations Office

rotational (exposure geometry)

radiation protection program

relative response factor

radiation work permit

shallow dose equivalent

statement of work

total effective dose

tissue-equivalent proportional counter

thermoluminescent

thermoluminescent dosimeter

time-temperature profile

uninterruptible power supply

ultraviolet

Digital Equipment Corporation VAX Computer Operating Environment whole body

Washington Closure Hanford

Westinghouse Hanford Company

year to date 


\section{CONTENTS}

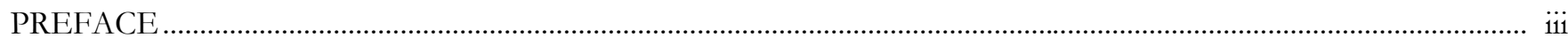

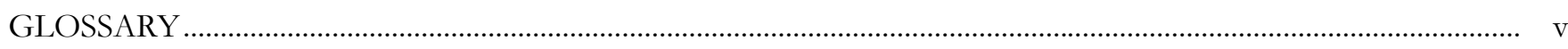

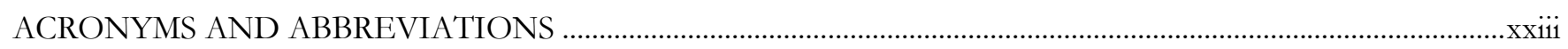

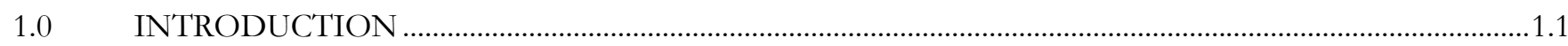

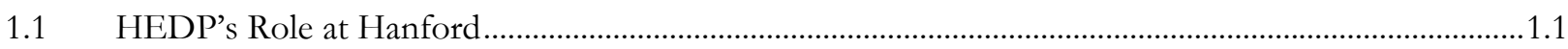

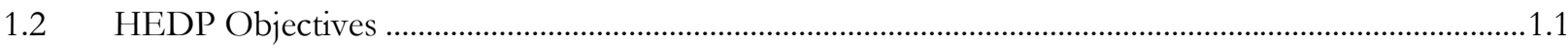

$2.0 \quad$ HANFORD EXTERNAL DOSIMETRY PROGRAM ................................................................................

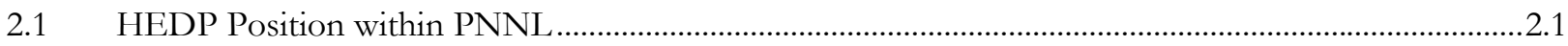

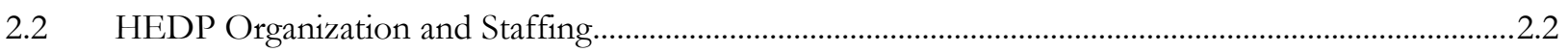

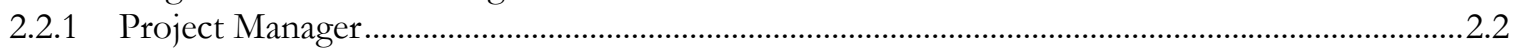

2.2.2 Technical Manager ....................................................................................................................2.2

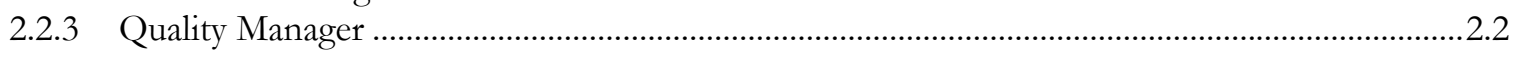

2.2.4 Dosimetry Professional Staff...................................................................................................2.2

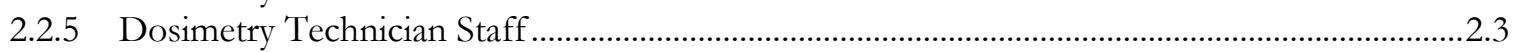

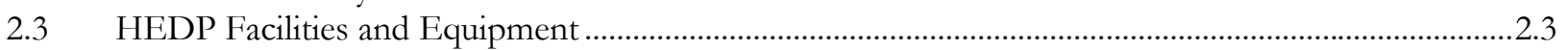

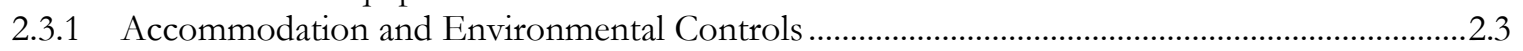

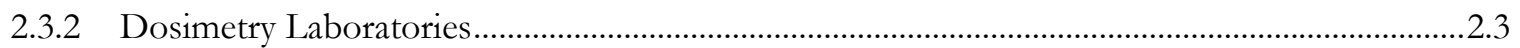

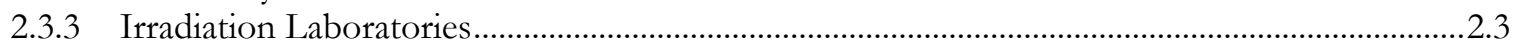

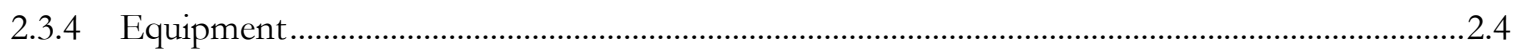

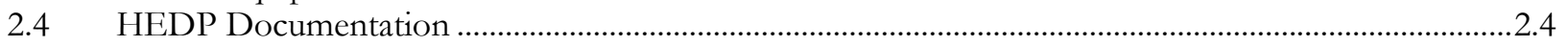

2.5 HEDP Functional Relationship with DOE and DOE Contractors ....................................................2.5

2.5.1 Hanford Personnel Dosimetry Advisory Committee .....................................................................2.5

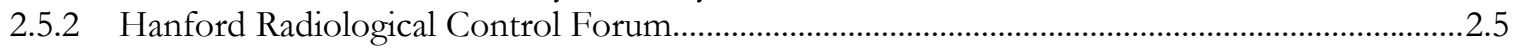

2.5.3 Hanford Contractor Work Agreements..................................................................................................

2.5.4 Non-Hanford Contractor Work Agreements ..................................................................................... 2.6

2.5.5 DOE Programmatic Assessments and Oversight.............................................................................

2.6 Field Dosimetry Practices and Contractor Responsibilities......................................................................2.6

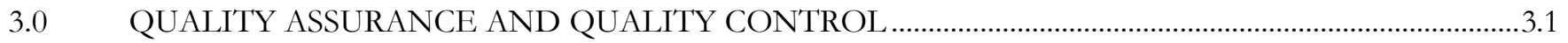

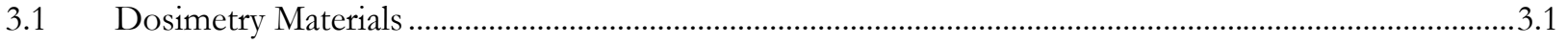

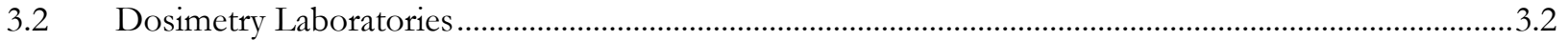

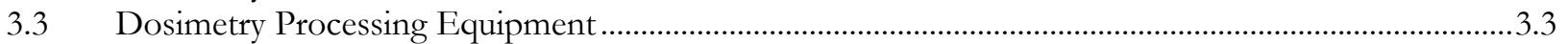

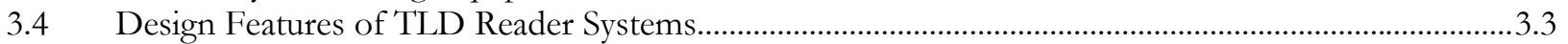

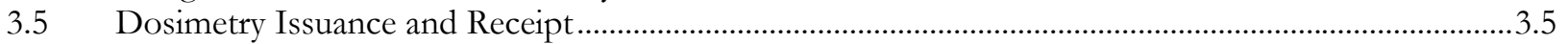

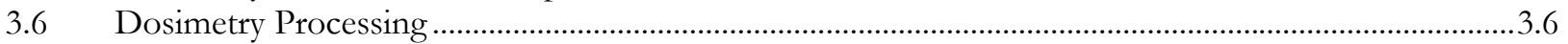

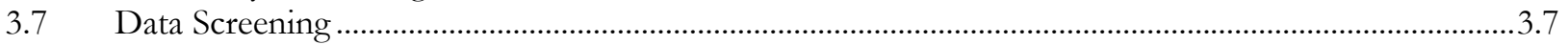

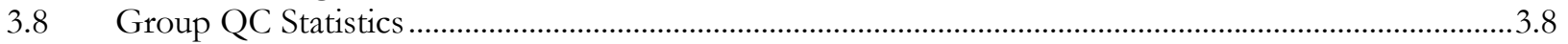

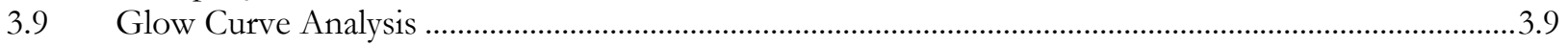

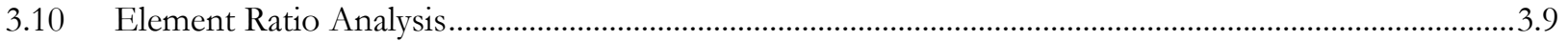

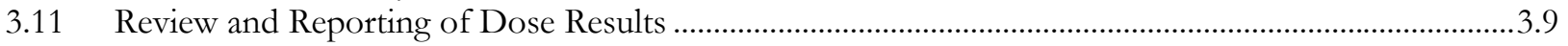

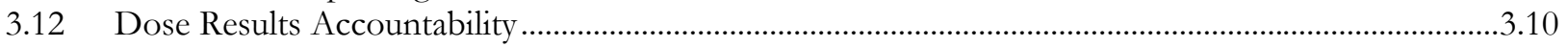

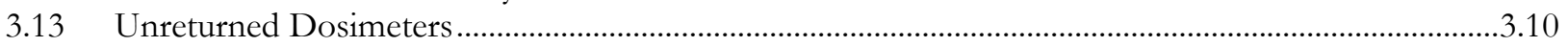

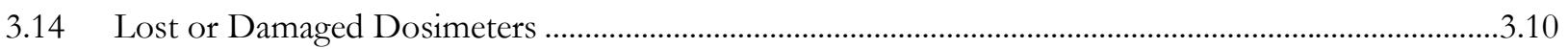

3.15 "High" Dose Results.....................................................................................................................11

3.16 Abnormal Dosimeter Results - Note Codes .........................................................................................11 


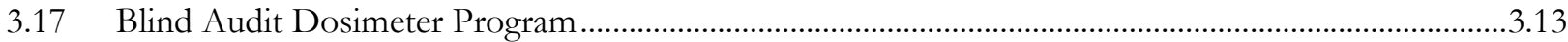

3.18 Dosimetry Processing Quality Assurance Reports.......................................................................................13

3.19 External Dosimetry Program Records .......................................................................................................13

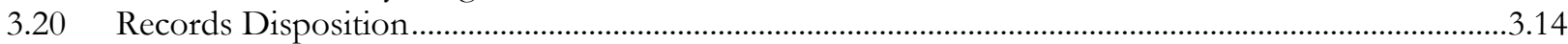

3.21 Other Quality Assurance Topics...............................................................................................................15

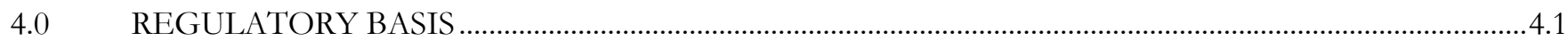

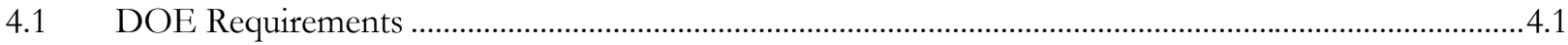

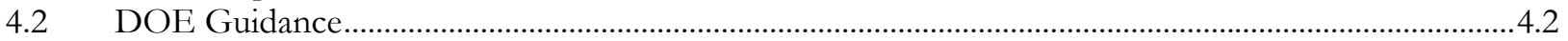

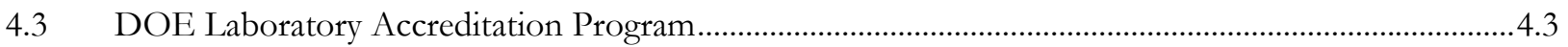

4.4 Operational and Protection Quantities .................................................................................................

4.4.1 How Operational Quantities and Dose Assessment Results are Stored in REX....................4.5

4.4.2 Relevant 10 CFR 835 Requirements............................................................................................ 4.7

4.4.3 How REX Fields are Used to Calculate 10 CFR 835 Protection Quantities.............................4.8

4.4.4 Mapping of Operational Quantities to 10 CFR 835 Protection Quantities ................................4.8

4.4.5 Inconsistencies with DOE Definition and Use of Protection Quantities...............................4.11

5.0 DOSIMETRY SYSTEM DESIGN, CALIBRATION, AND DOSE RESPONSE ....................................... 5.1

$5.1 \quad$ Characteristics of TLD Phosphors used in HEDP Dosimeters ................................................................ 5.2

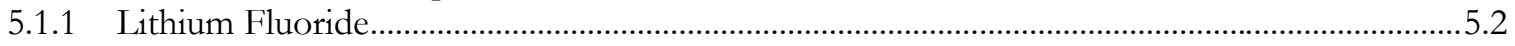

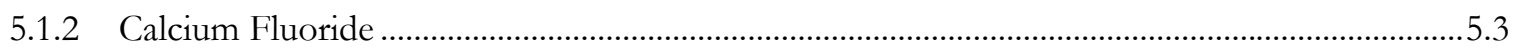

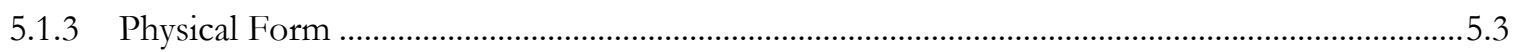

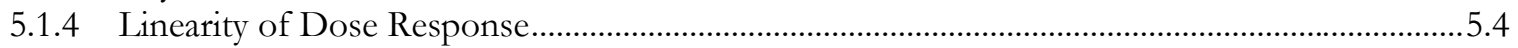

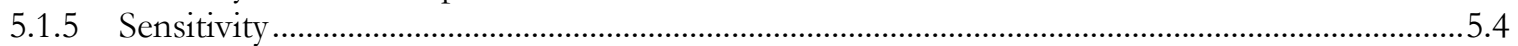

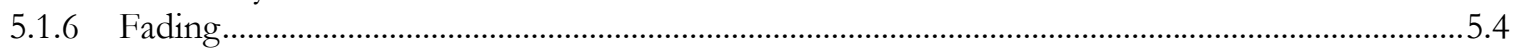

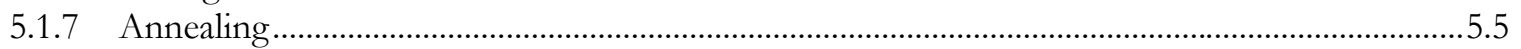

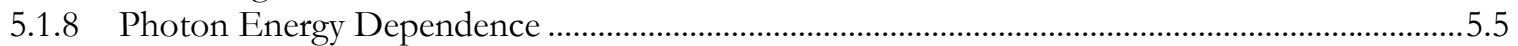

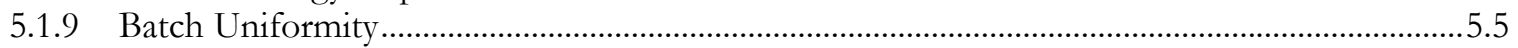

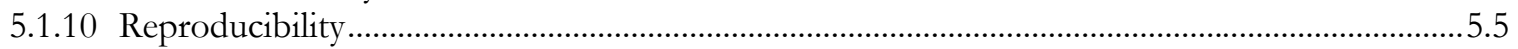

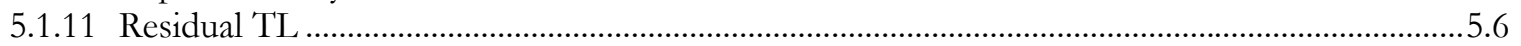

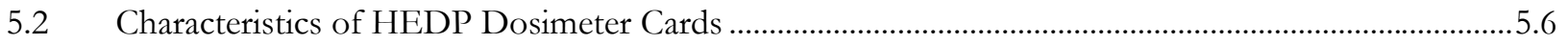

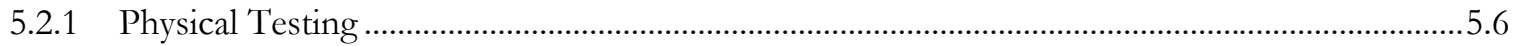

5.2.2 Unique Permanent Identification Number ....................................................................................

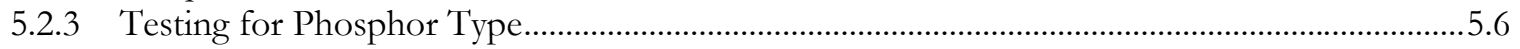

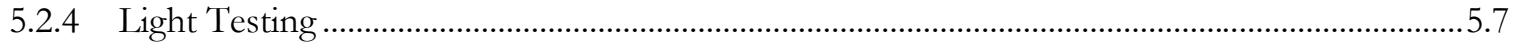

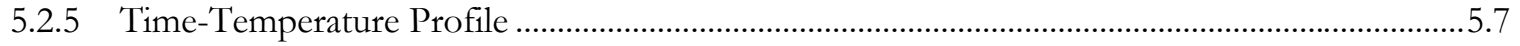

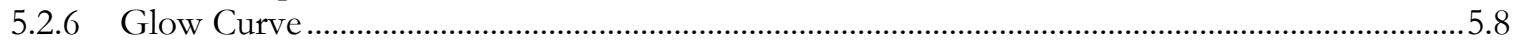

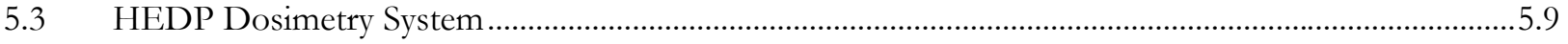

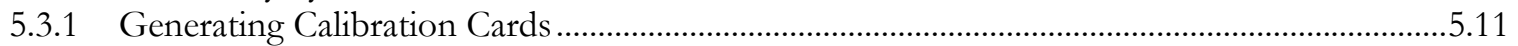

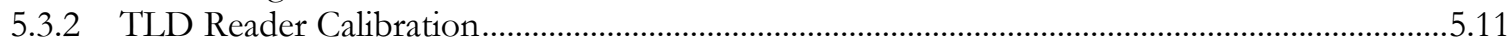

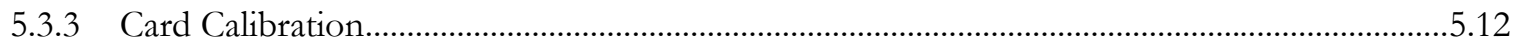

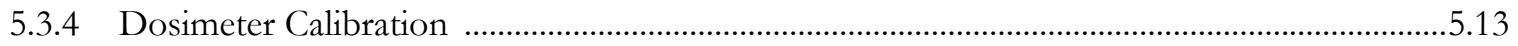

5.3.5 Calibrated Element Readings .......................................................................................................

5.3.6 Adjusted Element Readings...........................................................................................................

5.3.6.1 Models for Environmental Background ...........................................................................

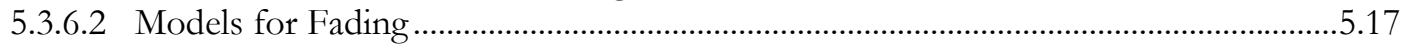

5.3.6.3 Models for Supralinearity ............................................................................................ 5.20

5.3.6.4 Calculation of Weighted Fading and Supralinearity Corrections $F_{i}$ and $S_{i} \ldots \ldots \ldots \ldots \ldots . . .5 .21$

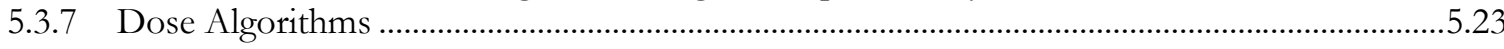

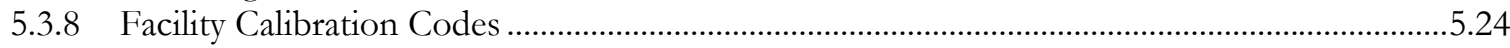

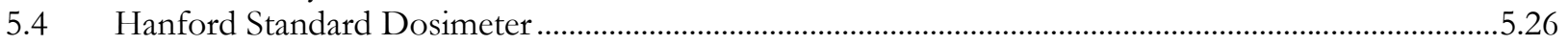

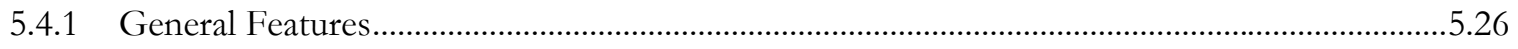

5.4.2 Dosimeter Assignment and Processing Protocol........................................................................5.26 


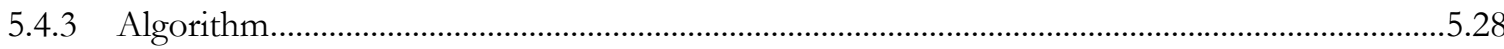

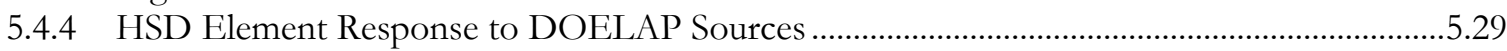

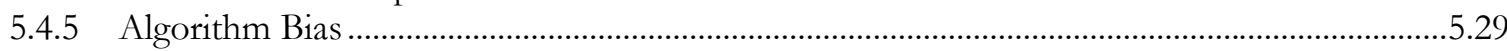

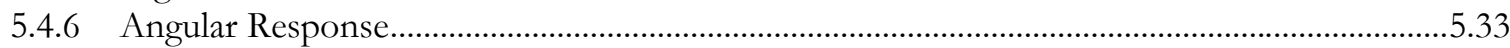

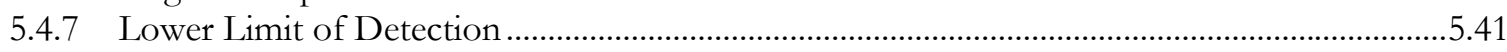

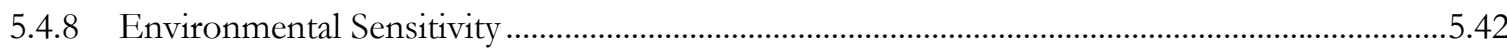

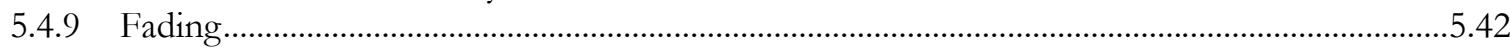

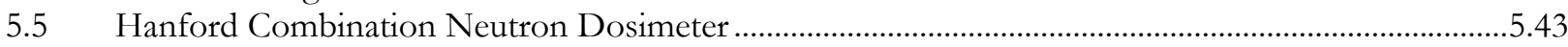

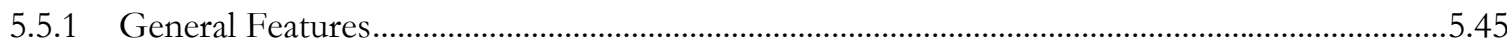

5.5.2 Dosimeter Assignment and Processing Protocol........................................................................46

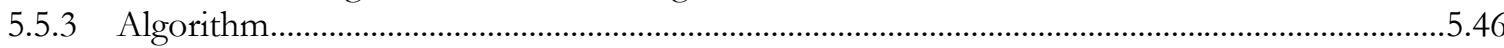

5.5.4 HCND Element Response to DOELAP Sources .......................................................................4.

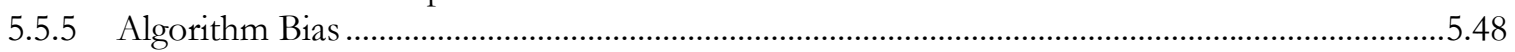

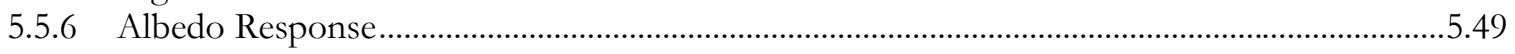

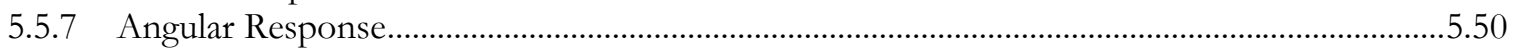

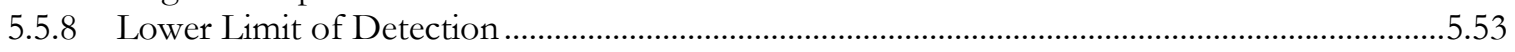

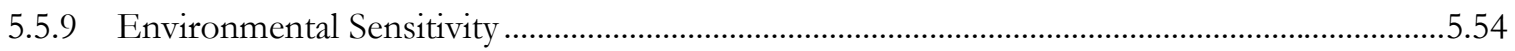

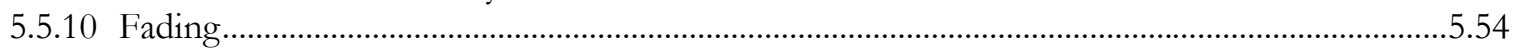

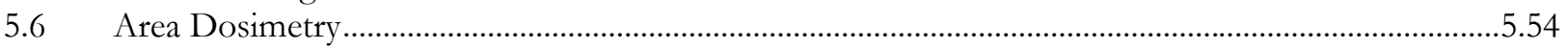

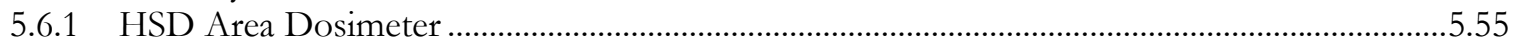

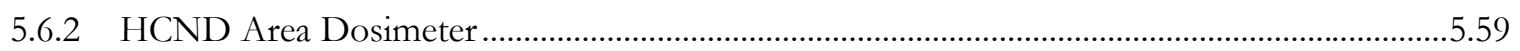

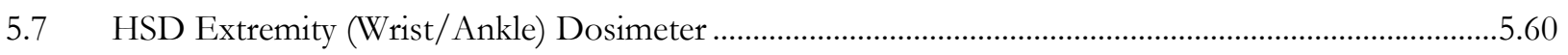

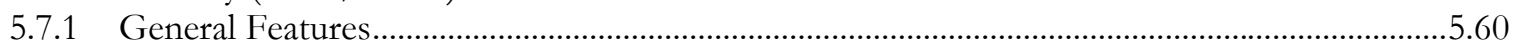

5.7.2 Dosimeter Assignment and Processing Protocol...........................................................................6.

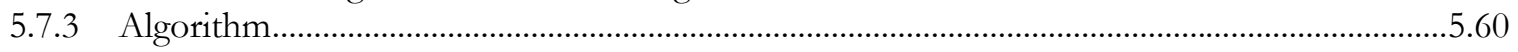

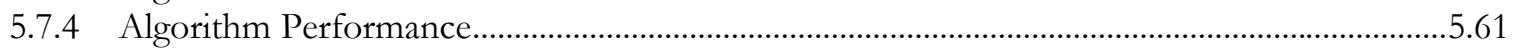

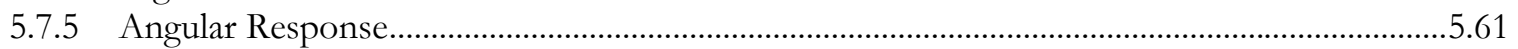

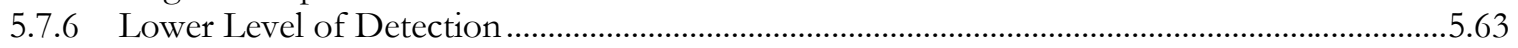

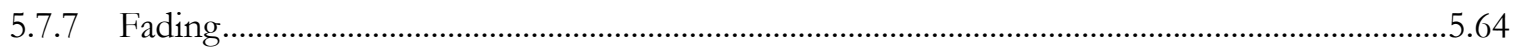

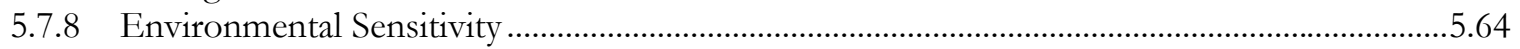

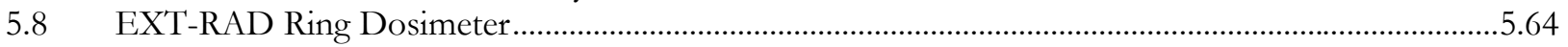

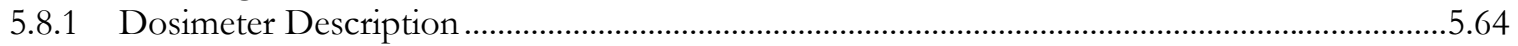

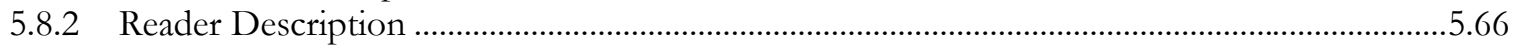

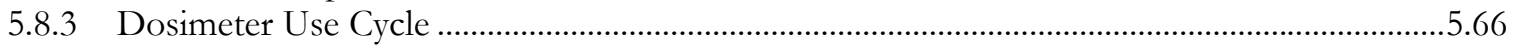

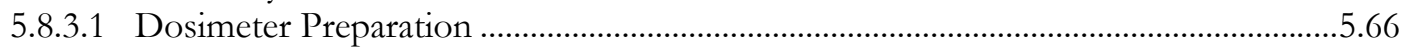

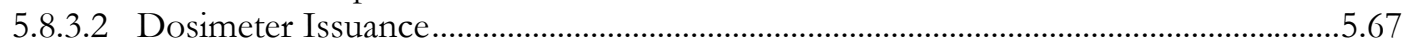

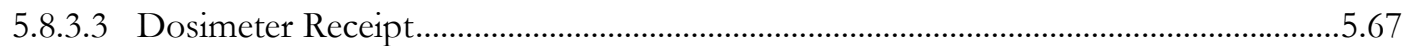

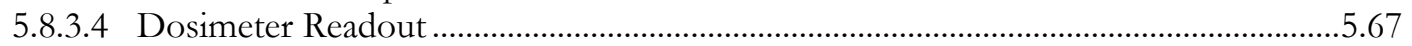

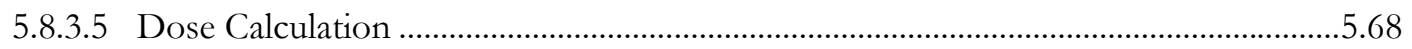

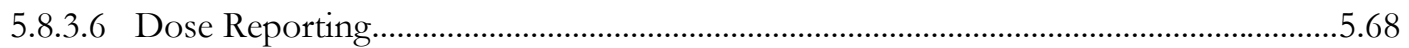

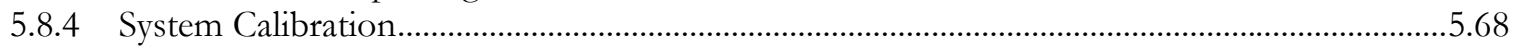

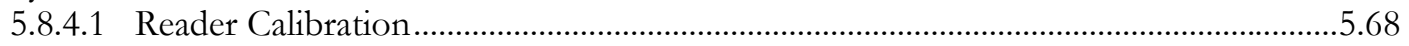

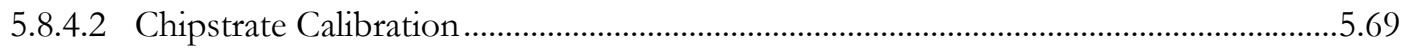

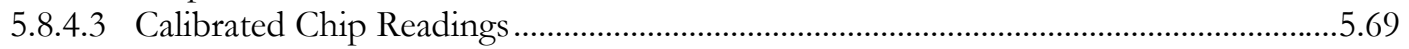

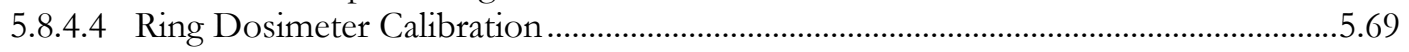

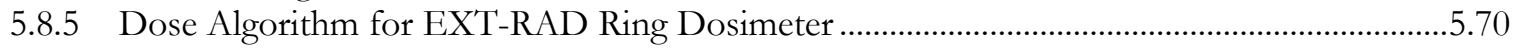

5.8.5.1 Shallow Personal Dose Equivalent................................................................................ 5.70

5.8.5.2 Facility Calibration Factor............................................................................................... 5.70

5.8.5.3 Adjusted Element Reading ……................................................................................... 5.70

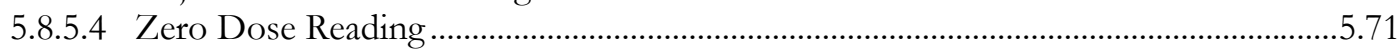

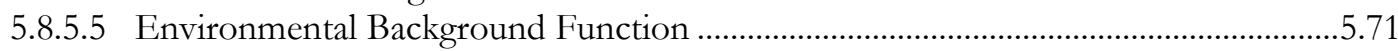

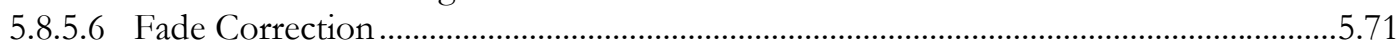

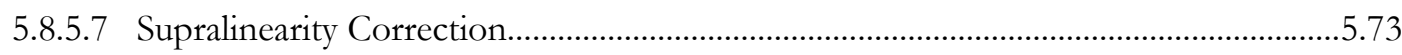




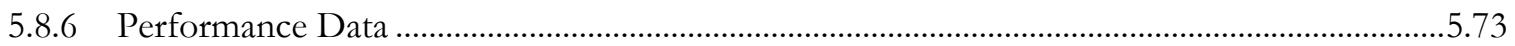

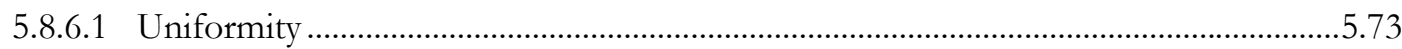

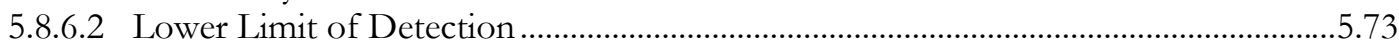

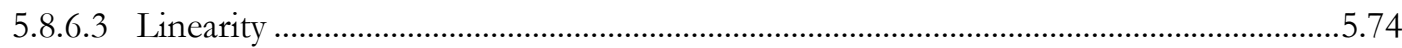

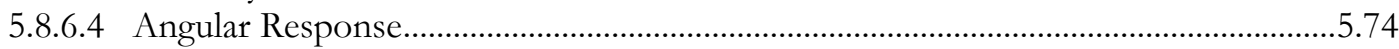

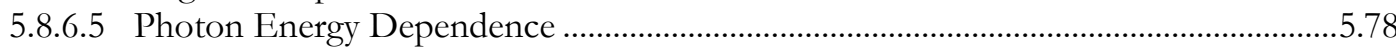

5.8.6.6 Beta Energy Dependence ................................................................................................. 5.79

5.8.7 Default Correction Factor for the EXT-RAD Ring ....................................................................5.79

5.8.8 EXT-RAD Ring Correction Factor for the Plutonium Finishing Plant....................................5.80

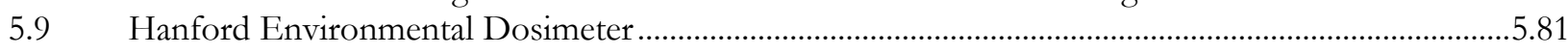

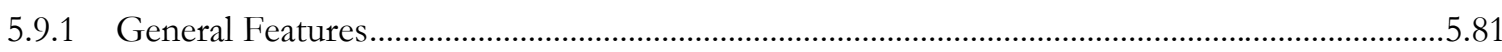

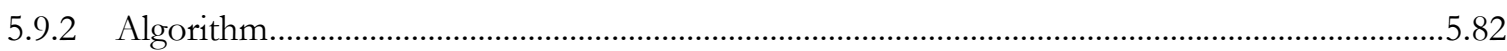

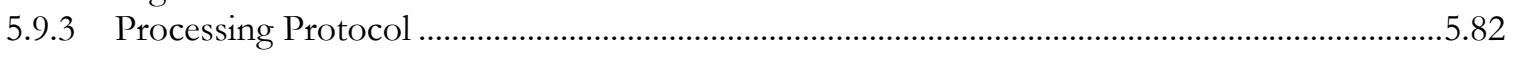

5.9.4 Energy Response ......................................................................................................................... 5.83

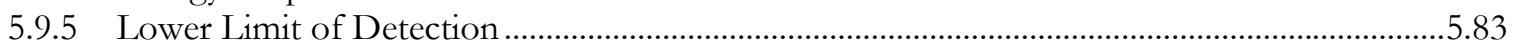

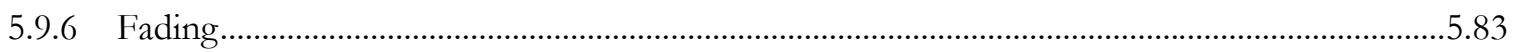

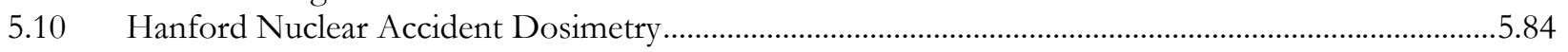

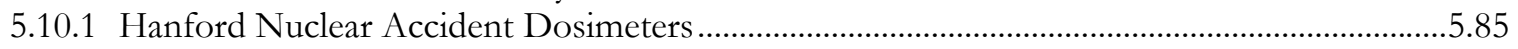

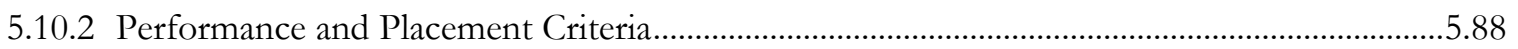

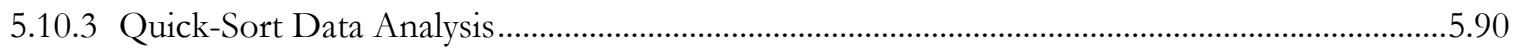

5.10.4 Analysis of Physical and Biological Samples ............................................................................5.92

5.10.5 Interpretation of Personnel Dosimeter Results After a Criticality Event ..................................5.93

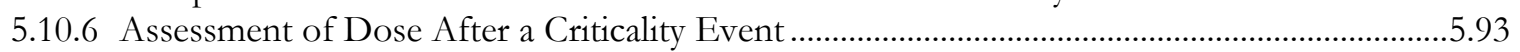

5.11 Useful Dose Range for Hanford Dosimeters ..........................................................................................5.94

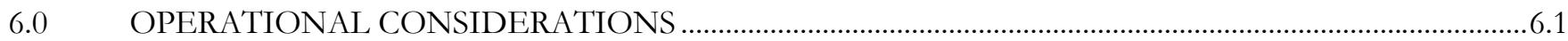

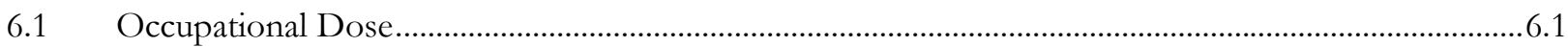

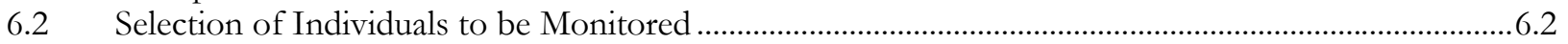

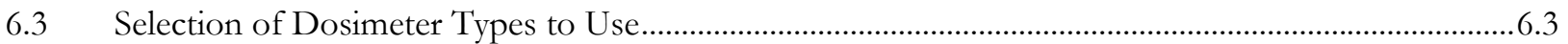

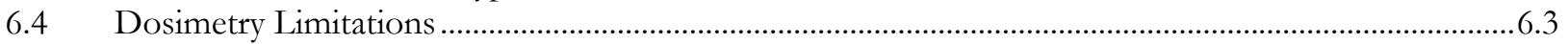

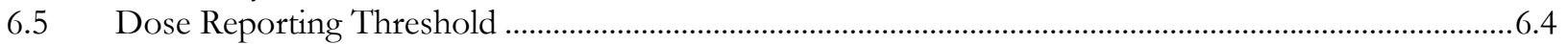

6.6 Dosimeter Exchange and Selection of Frequency …….........................................................................6.7

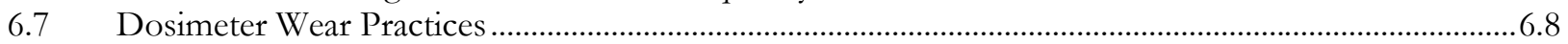

6.7.1 Dosimeter Use with Protective Clothing.....................................................................................6.10

6.7.1.1 Assessing the Potential for Under Recording Equivalent Dose to the Skin Using Knowledge of Average Beta Energy .................................................................6.12

6.7.1.2 Assessing the Potential for Under Recording Equivalent Dose to the Skin Using Survey Instruments ....................................................................................................

6.7.1.3 Exposure to Mixed Fission Product Activity in Sample Containers............................6.14

6.7.1.4 Exposure to Mixed Fission Product Contaminated Surfaces .......................................6.14

6.7.2 Dosimeter Use with Lead Aprons or Lead Vests in Uniform External Fields ........................6.17

6.8 When to Use Multiple Dosimeters for Work in Non-Uniform Fields .................................................6.17

6.8.1 Monitoring Workers with Multiple Whole Body Dosimeters..........................................................19

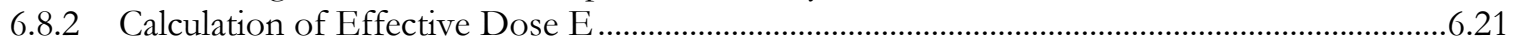

6.8.3 Assessment of Equivalent Dose to Skin, Lens of Eye, and Extremity

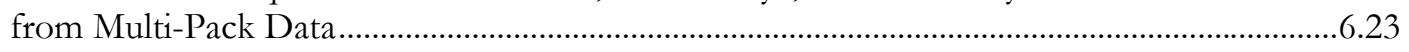

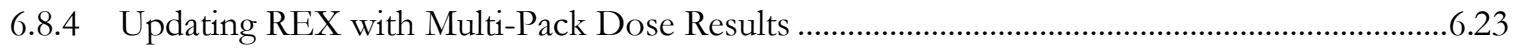

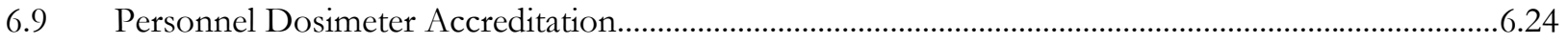

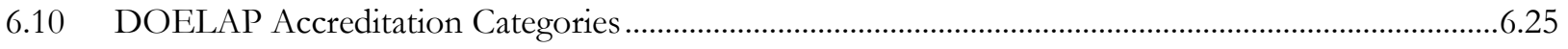

6.11 Radiation Types Not Covered by DOELAP Performance Testing......................................................6.28

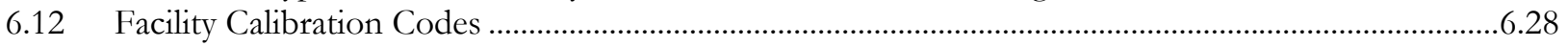

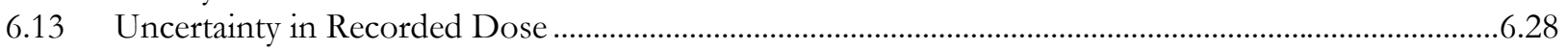

6.14 Approved Dosimeter Configurations.................................................................................................29 


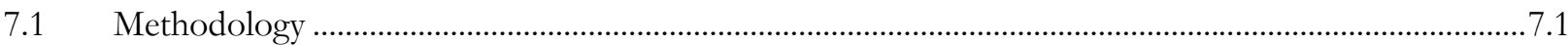

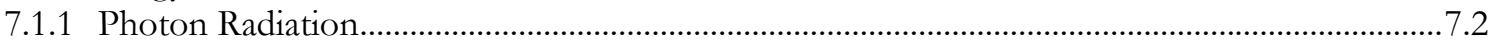

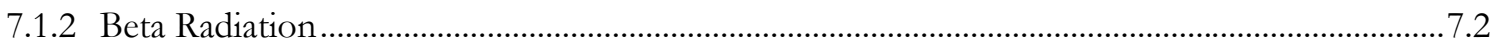

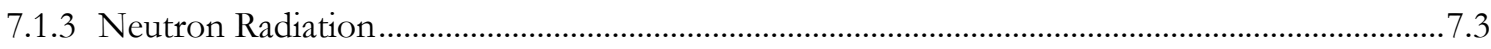

7.1.3.1 Multisphere Spectrometer ............................................................................................ 7.3

7.1.3.2 Tissue Equivalent Proportional Counter (TEPC) ……………………….......................... 7.4

7.2 Hanford Beta Radiation Measurements ......................................................................................... 7.5

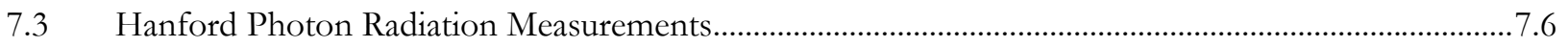

7.4 Hanford Neutron Radiation Measurements...........................................................................................

7.5 Hanford Environmental Radiation Measurements ...............................................................................

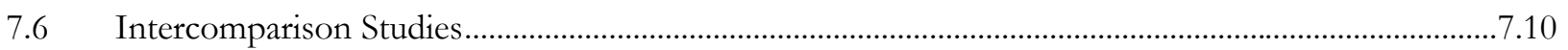

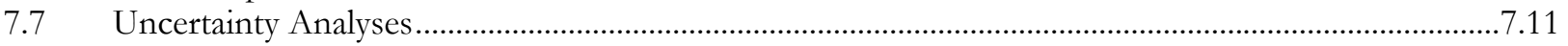

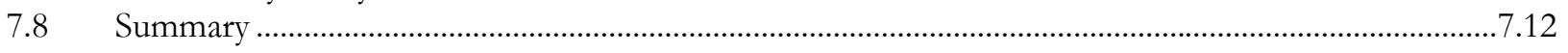

8.0 ASSESSMENT OF NON-UNIFORM DOSE FROM CONTAMINATION ON SKIN OR

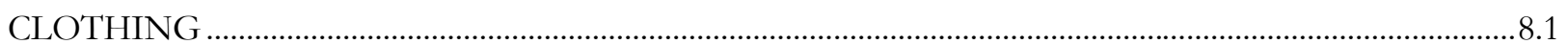

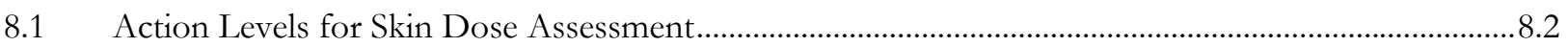

8.2 Assessment of Dose to Skin or Extremities from Contamination ............................................................ 8.4

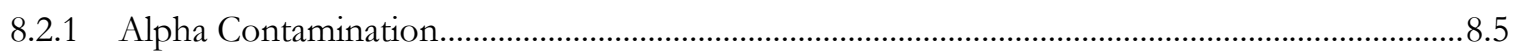

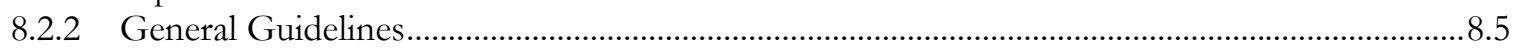

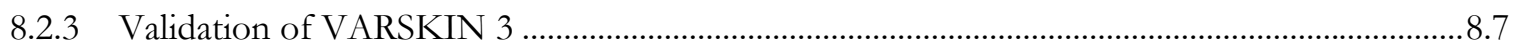

8.2.4 Considerations of the Air/Tissue Interface When Using VARSKIN 3 ....................................10

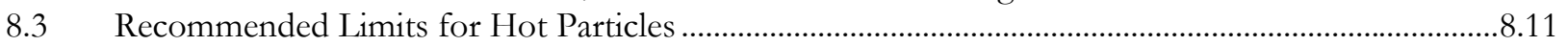

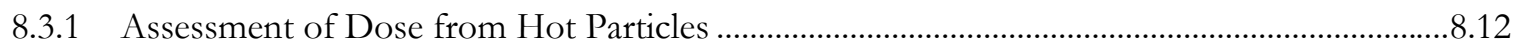

8.3.2 Recommended Action Levels for Hot Particle Control................................................................15

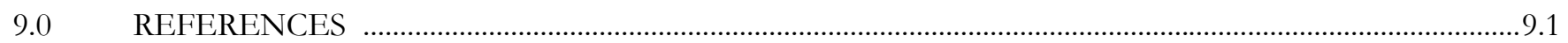




\section{FIGURES}

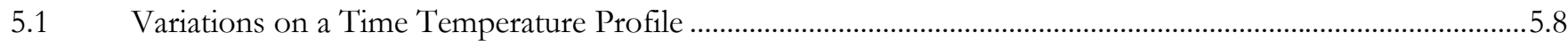

Typical LiF glow curve with standard TTP

5.3 Buildup of Environmental Background Signal in HSD Shallow Dose Element............................................5.15

5.4 Buildup of Environmental Background Signal in HSD Deep Dose Element ...............................................5.16

5.5 Post Irradiation Fade Functions Used for Hanford Dosimetry Materials ........................................................5.18

5.6 Error in Default Fade Correction When Entire Exposure Occurs on First Day of Use Cycle .....................5.19

5.7 Error in Default Fade Correction When Entire Exposure Occurs on Last Day of Use Cycle........................5.19

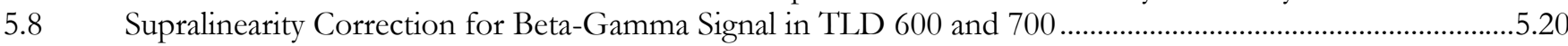

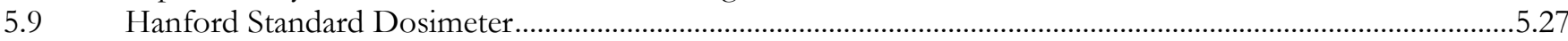

5.10 Irradiation Setup for Dosimeter Angular Response Evaluation.......................................................................5.34

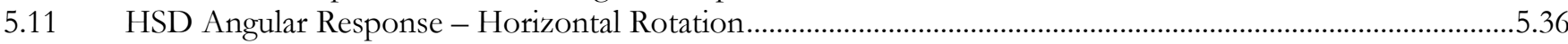

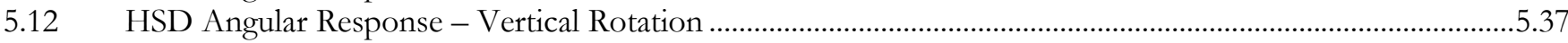

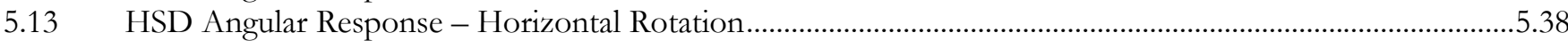

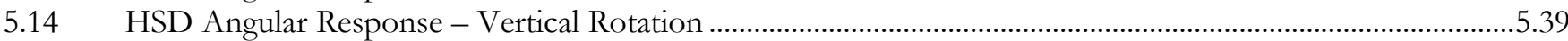

$5.15 \quad$ HSD Neutron Angular Response - Horizontal Rotation ................................................................................5.40

$5.16 \quad$ HSD Neutron Angular Response - Vertical Rotation..........................................................................................

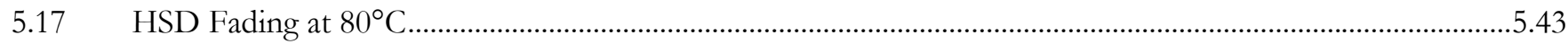

5.18 Hanford Combination Neutron Dosimeter with PNAD ...................................................................................4.

5.19 Hanford Combination Neutron Dosimeter without PNAD ..........................................................................5.44

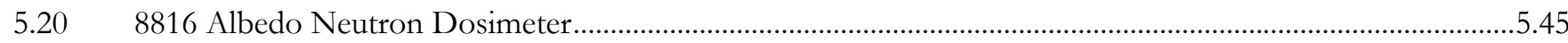

$5.21 \quad$ Measured Albedo Response of 8816 Neutron Dosimeter .................................................................................49

$5.22 \quad$ HCND Shallow Dose Angular Response - Horizontal Rotation .....................................................................5.51

$5.23 \quad$ HCND Shallow Dose Angular Response - Vertical Rotation ………............................................................5.51

$5.24 \quad$ HCND Deep Dose Angular Response - Horizontal Rotation...........................................................................5.52

$5.25 \quad$ HCND Deep Dose Angular Response - Vertical Rotation..............................................................................5.52

5.26 HSD Extremity Shallow Dose Angular Response - Horizontal Rotation ........................................................5.62

5.27 HSD Extremity Shallow Dose Angular Response - Vertical Rotation .................................................................63

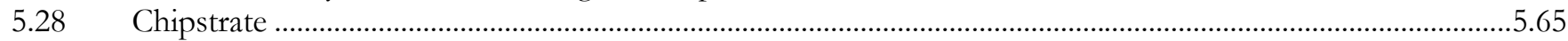

$5.29 \quad$ Illustration of Chipstrate Sealed in Vinyl Pouch ...............................................................................................65

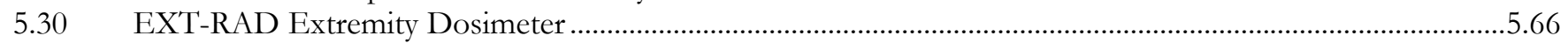

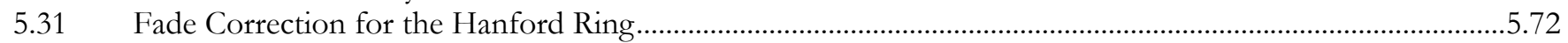

5.32 EXT-RAD Ring Shallow Dose Angular Response - Horizontal Orientation.................................................5.76

5.33 EXT-RAD Ring Shallow Dose Angular Response - Vertical Orientation ........................................................5.76

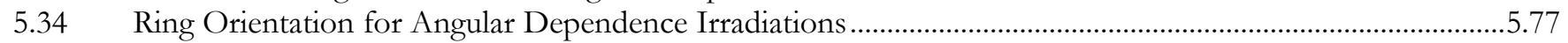

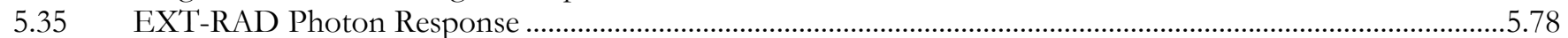

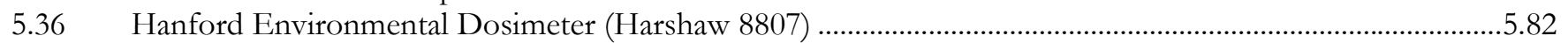

5.37 Hanford Environmental Dosimeter Energy Response (TLD 700) ...............................................................5.84

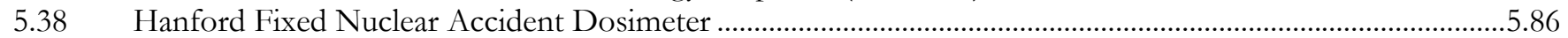

$5.39 \quad$ Hanford Personal Nuclear Accident Dosimeter ...................................................................................................

6.1 Measured Shallow Dose Attenuation by Protective Clothing for a ${ }^{85} \mathrm{Kr}$ Beta Source ....................................6.11

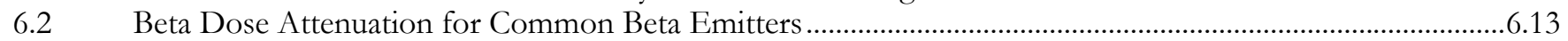

6.3 Calculated Shallow Dose Attenuation for ${ }^{90} \mathrm{Sr},{ }^{90} \mathrm{Y},{ }^{137} \mathrm{Cs}$ and ${ }^{137 \mathrm{~m} B a}$...........................................................15

6.4 Calculated Shallow Dose Attenuation for Mixtures of ${ }^{90} \mathrm{Sr},{ }^{90} \mathrm{Y},{ }^{137} \mathrm{Cs}$ and ${ }^{137 \mathrm{mBa}}$

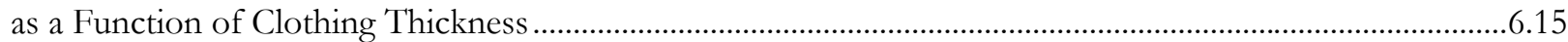

6.5 Calculated Shallow Dose Attenuation for Various Clothing Thicknesses as a Function

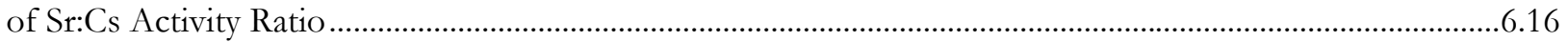

6.6 Shallow Dose Attenuation as a function of Clothing Thickness and Sr:Cs Activity Ratio ..............................6.16

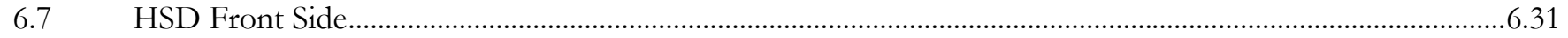

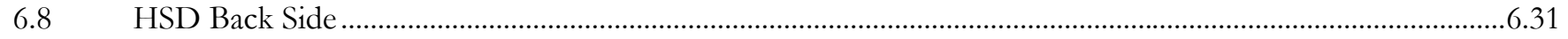

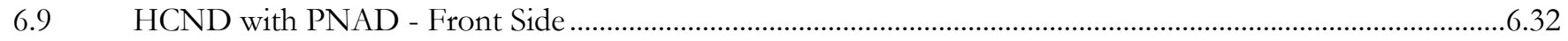




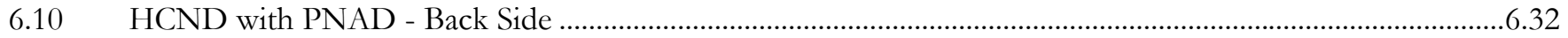

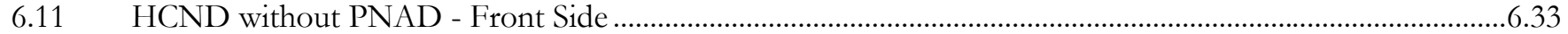

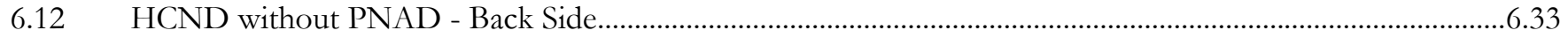

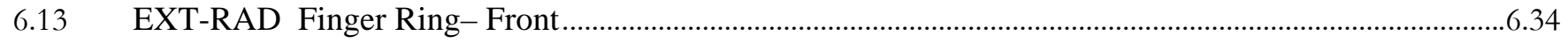

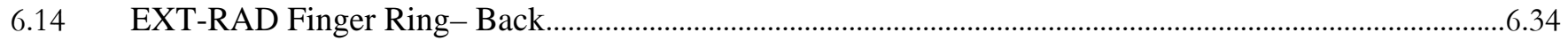

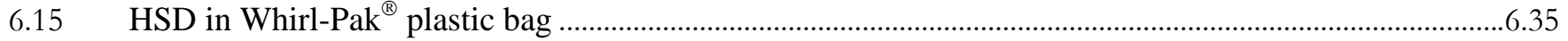

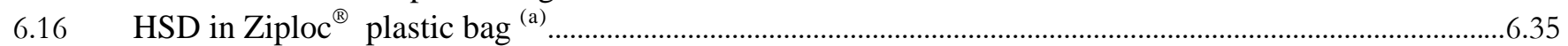

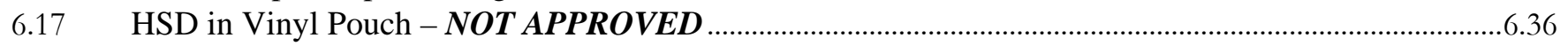

(a) Ziploc ${ }^{\circledR}$ is a registered trademark of S.C. Johnson \& Son, Inc. All rights reserved. 


\section{TABLES}

4.1 Storage of Dosimeter Results and Dose Assessment Information in REX Fields ...........................................4.6

4.2 Summation of Fields in REX to Obtain Protection Quantities .......................................................................4.9

4.3 Mapping of Dosimeter Results and Dose Assessment Results to 10 CFR 835 Protection Quantities...........4.10

5.1 Trace Elements in Lithium Fluoride Thermoluminescent Dosimeters..............................................................5.2

5.2 Typical Parameters for Reader Processing Setup.............................................................................................5.7

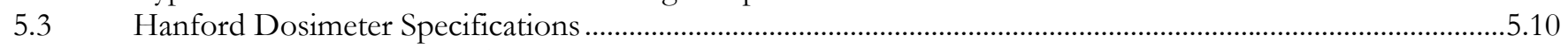

5.4 Parameters for Background Functions .......................................................................................................5.15

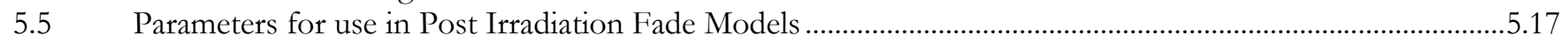

5.6 HSD Element Response to DOELAP Sources ..................................................................................................5.30

5.7 HSD Algorithm Shallow and Deep Dose Response .....................................................................................5.31

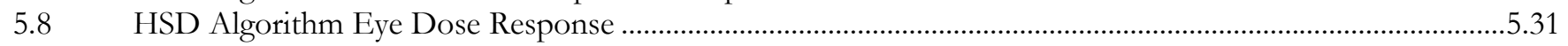

$5.9 \quad$ HSD Algorithm Neutron Deep Dose Response …….................................................................................5.32

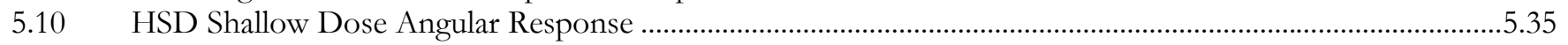

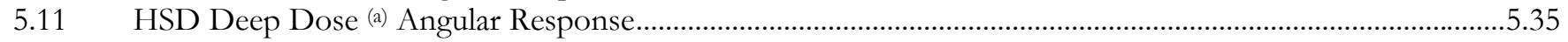

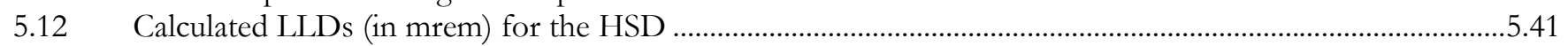

5.138816 Neutron Dosimeter Element Response to DOELAP Sources ................................................................5.47

5.148816 Neutron Dose Response with General Use Algorithm............................................................................4.48

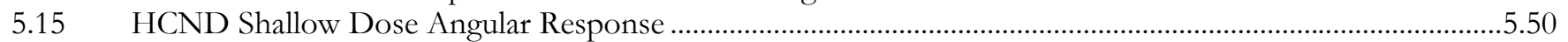

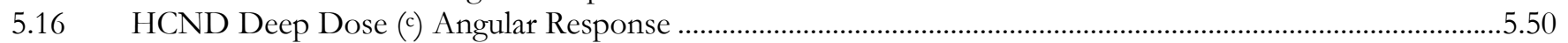

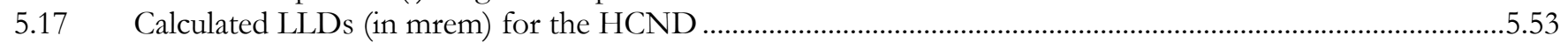

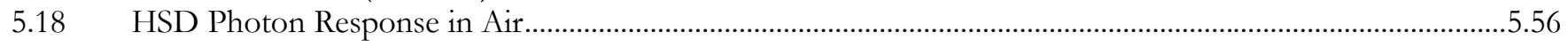

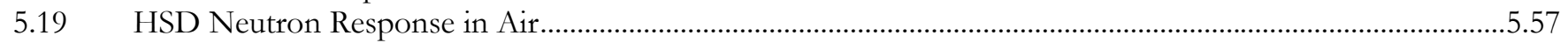

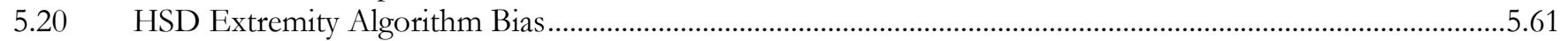

$5.21 \quad$ HSD Extremity Dosimeter Angular Response ....................................................................................................62

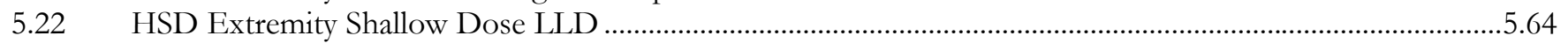

$5.23 \quad$ Parameters for use in Chipstrate Post Irradiation Fade Model ..........................................................................5.72

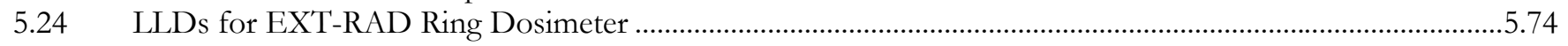

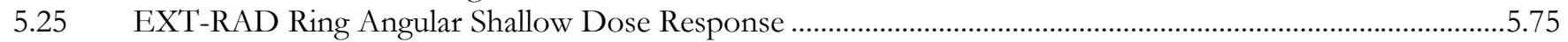

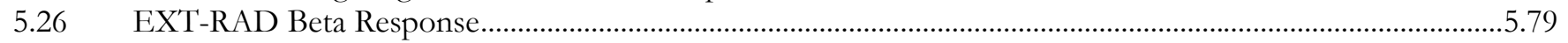

5.27 Hanford Environmental Dosimeter Time-Temperature Profiles ......................................................................5.83

$5.28 \quad$ Materials and Approximate Dimensions of FNAD Components ......................................................................5.87

$5.29 \quad$ Materials and Approximate Dimensions of PNAD Components .....................................................................5.88

$5.30 \quad$ Survey Instrument Readings on the Body after an Accident .......................................................................5.91

$5.31 \quad$ Maximum Measurable Dose for Hanford Dosimeters .......................................................................................5.95

5.32 Maximum Measurable Dose for HSD at Various Depths …….......................................................................5.96

6.1 Summary of Dosimeter Applications and Limitations $\left({ }^{(}\right)$…................................................................................6.5

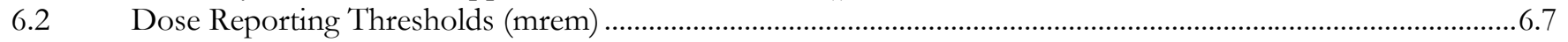

6.3 HSD/HCND Deep Dose Response on a Phantom Oriented 180 from Source ..........................................6.10

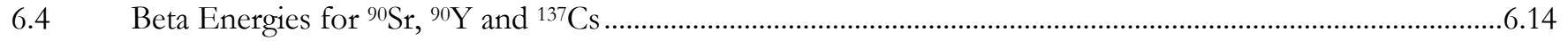

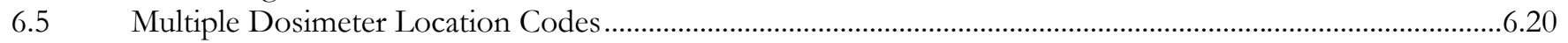

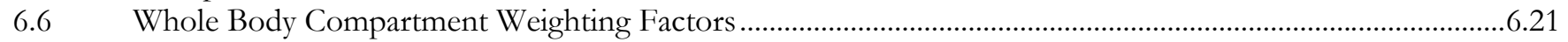

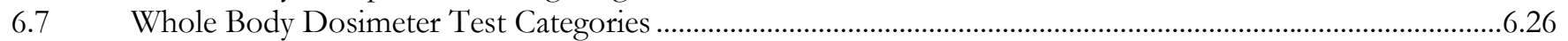

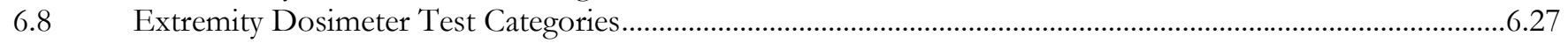


8.1 Portable Survey Instrument Action Levels for Skin Dose Assessment ...........................................................8.2

8.2 Measured Instrument Response and Calculated Dose for Small $\left(0.2 \mathrm{~cm}^{2}\right)$ Radiation Sources ........................8.4

8.3 Measured Instrument Response and Calculated Dose for Large $\left(225 \mathrm{~cm}^{2}\right)$ Radiation Sources........................8.4

8.4 Comparison of Skin Dose Conversion Factors for Area Contamination..........................................................8.8

8.5 Comparison of Skin Dose Conversion Factors for Point Source Contamination .............................................8.9

8.6 Measured Eberline Model RO-2 and RO-3B Ionization Chamber Response and Calculated

Dose for Point $\left(<2 \mathrm{~mm}^{2}\right)$ Radiation Sources ...........................................................................................................

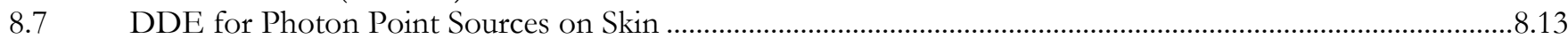


THIS PAGE INTENTIONALLY LEFT BLANK 


\subsection{Introduction}

The Hanford External Dosimetry Program (HEDP) has been an integral component of radiation protection at Hanford since its inception in 1944 (Wilson 1987). Since 1944, a centralized site-wide dosimetry system operated by HEDP or its predecessor organizations has been used to measure dose to Hanford workers and the Hanford environment. The HEDP complies with the DOE Laboratory Accreditation Program (DOELAP) standard (DOE 1986a) and DOELAP handbook (DOE 1986b) requirements and has been accredited under DOELAP since 1988.

\subsection{HEDP's Role at Hanford}

The HEDP provides consistent personnel, area, environmental and nuclear accident dosimetry services and technical support to the DOE Richland Operations Office (RL), DOE Office of River Protection (ORP), DOE Pacific Northwest Site Office (PNSO), and DOE contractors under the management of these offices.

Funding for HEDP is currently provided through cost reimbursement contracts with the DOE site offices and prime contractors, plus separately funded technical support tasks performed on a cost reimbursement basis. HEDP objectives and scope of work are defined through DOE program oversight and statements of work (SOW) with each contractor. HEDP's mission is to provide accurate, technically defensible personnel dose results for DOE and DOE contractors at Hanford, and to support the Radiation Protection Program (RPP) of each contractor. HEDP supports the RPP of each contractor by providing DOELAP accredited dosimetry services, technical basis documentation, and technical support as requested. HEDP currently maintains DOELAP accreditation and technical basis documentation for a centralized external dosimetry program shared by all DOE contractors at the Hanford site.

\subsection{HEDP Objectives}

External dosimetry is an integral part of most radiation protection programs. Viewing radiation dosimetry as an essential part of radiation protection, the National Council on Radiation Protection and Measurements Report No. 114, Maintaining Radiation Protection Records (NCRP 1992) identifies four major reasons for a radiation dosimetry program as follows:

- provide information allowing evaluation of the radiation safety program to ensure effective program operation

- $\quad$ provide evidence for regulatory compliance

- $\quad$ provide data for epidemiological studies

- $\quad$ provide information for making or contesting claims for radiationinduced injury 
HEDP objectives are consistent with these NCRP objectives. HEDP objectives may be summarized as follows:

- Provide accurate and technically defensible personnel, area, environmental and nuclear accident dose results

- Conduct the external dosimetry program in a manner that is compliant with applicable DOE regulations and direction, and consistent with applicable guidance

- Document policies, procedures and technical bases in sufficient detail to ensure defensibility of the dose of record for Hanford workers and to demonstrate compliance with applicable DOE regulations and direction, and consistency with applicable standards and guidance

- Provide and document laboratory and field measurements that establish the accuracy of dosimeter response under field conditions

- Maintain DOELAP accreditation for the Hanford Site

- Develop and maintain technical basis documentation for the Hanford Site

- Coordinate a consistent, shared dosimetry program for Hanford Site contractors through participation in the Hanford Personnel Dosimetry Advisory Committee (HPDAC)

- $\quad$ Support the RPP of each Hanford contractor as specified in SOWs

- $\quad$ Provide services that are cost effective and meet the needs of all contractors

- Participate in intercomparison programs in personnel, extremity, and nuclear accident dosimetry to demonstrate competency in areas outside of DOELAP

- Develop and implement improved technology when benefits clearly outweigh costs, or existing technology is inadequate to provide accurate dose results or support contractor objectives

- Ensure program conformance with applicable recommendations of the Health Physics Society (HPS), American National Standards Institute (ANSI), NCRP, and the ICRP whenever feasible. 


\subsection{Hanford External Dosimetry Program}

The HEDP provides personnel, area, environmental, and nuclear accident dosimetry services for DOE personnel and contractors at Hanford and other DOE sites. The HEDP staff is responsible for conducting these activities in compliance with applicable DOE requirements and standards of good professional practice. Major areas of effort in the conduct of the HEDP are as follows:

- routine and special dosimeter processing and dose reporting

- dosimeter tracking and accountability

- dosimetry materials procurement and acceptance testing

- dosimeter and dosimetry system calibration

- equipment maintenance and calibration

- dosimeter processing quality control and dose results quality assurance

- verification of dosimeter response in work environments

- procedure development and maintenance

- $\quad$ software development and maintenance

- algorithm development and supporting technical studies

- documentation and record keeping

- performance testing and accreditation

\subsection{HEDP Position within PNNL}

HEDP is organized as a program within Radiation and Health Technology (R\&HT) Technical Group which functions within the Radiological and Nuclear Science and Technology Division, which in turn functions within the Energy and Environment Directorate (EED) at the Pacific Northwest National Laboratory (PNNL).

The R\&HT technical group provides Hanford services for external dosimetry, internal dosimetry, in vivo and in vitro bioassay, instrument calibration, and radiological records. In addition, R\&HT operates a National Institute of Standards and Technology (NIST) secondary calibration laboratory for ionizing radiation and a laboratory for type testing of radiation instruments and electronic dosimeters. 


\subsection{HEDP Organization and Staffing}

PNL-MA-841 Hanford External Dosimetry Program Procedures Manual describes HEDP roles and respective responsibilities in detail. An overview of critical roles is provided in the following subsections.

\subsubsection{Program Manager}

The HEDP Program Manager is responsible for overall administration of the program within budgetary constraints and in a manner consistent with contractual obligations. The program manager conducts line management of HEDP staff members including annual competency reviews. The program manager assigns roles and responsibilities for each staff member, prioritizes and assigns tasks, tracks progress, manages the budget, and ensures work is conducted in compliance with DOE requirements and within the scope of service contracts with Hanford contractor and other clients.

\subsubsection{Technical Manager}

The HEDP Technical Manager is responsible for HEDP technical practices including maintenance of the Technical Basis and Procedures Manuals, and is responsible for ensuring compliance with the conditions and criteria for accreditation. The Technical Manager is the point of contact for DOELAP correspondence, receives proficiency testing materials and reports, and is the contact for onsite assessments. The Technical Manager is a professional experienced in applied radiation dosimetry who is knowledgeable in the design and operation of the HEDP system. The Technical Manager has the technical competence and the supervisory capability to direct the work of professionals and technicians in the dosimetry area.

\subsubsection{Quality Manager}

The HEDP Quality Manager is responsible for the quality system, including the Quality Manual, and its implementation. The Quality Manager reports to the R\&HT Technical Group manager, representing the highest level of management at which decisions are made regarding dosimetry laboratory policy or resources.

\subsubsection{Dosimetry Professional Staff}

HEDP professional staff members are assigned several quality affecting roles. These roles include Dosimetrist, Training Coordinator, Computer Systems Administrator, etc., as defined in PNL-MA-841 Hanford External Dosimetry Program Procedures Manual. Proper conduct of these roles is crucial to the HEDP quality system. Staff assignments are made based on HEDP needs and staffing resources. The HEDP Program Manager makes these assignments with concurrence from the R\&HT Manager. 


\subsubsection{Dosimetry Technician Staff}

HEDP dosimetry technicians are dedicated to external dosimetry activities. These technicians are qualified in the respective procedures contained in PNL-MA-841 Hanford External Dosimetry Program Procedures Manual, and work under the technical direction of the Technical Manager. Training and qualification of HEDP technicians consists of formal classroom training, assigned reading, and job specific training (JST).

\subsection{HEDP Facilities and Equipment}

The R\&HT staff occupies the 318 Radiological Calibrations Facility in the Hanford 300 Area near Richland Washington. This facility contains laboratories for dosimeter irradiation, portable radiological instrument calibration and repair, instrument environmental and type testing, and the HEDP dosimetry laboratories, along with staff offices. These laboratories, equipment, and staff members support a wide range of Hanford, national and international client programs. The 318 facility includes a NVLAP accredited calibration laboratory for ionizing radiation. Irradiations for NVLAP and DOELAP personnel and extremity dosimetry performance testing, and for contracted dosimeter and instrument irradiation services, are performed in this facility.

\subsubsection{Accommodation and Environmental Controls}

The 318 Facility, totaling about 30,000 square feet, contains the original threestory 318 Building, four additions to the original building, and an adjoining trailer addition. The 318 Facility is primarily a laboratory complex with controlled ventilation, heating, cooling, humidity, etc., in accordance with good professional practice. Laboratories can be broadly divided into dosimetry and irradiation laboratories as described in the following subsections.

\subsubsection{Dosimetry Laboratories}

HEDP dosimetry laboratories generally occupy approximately 7,000-square feet in the south end of the building, an area dedicated to non-radiological designated laboratories. The laboratory and office space in the end of the building was designed to house the HEDP with dedicated, uninterruptible power supply (UPS) supported electrical circuits for seven reader systems.

\subsubsection{Irradiation Laboratories}

Irradiation laboratories generally occupy the basement and first floor of the original 318 Building, and selected laboratories for portable instrument testing and calibrations of the 318 Facility. All high-level irradiation capabilities are located within the original 318 Building. Irradiation capabilities covering a broad range are available within the 318 Facility. These capabilities have been used to evaluate and document response characteristics of HEDP dosimeters. Thermoluminescent Dosimeter (TLD) area monitors throughout the building are used to ensure that the use of irradiation sources does not produce measurable dose in the dosimetry labs and other non-radiological areas. 


\subsubsection{Equipment}

HEDP dosimetry equipment is for the most part commercially procured and offers the best performance obtainable in thermoluminescence dosimetry systems. Primary and backup equipment exists for all critical program activities including annealing, bar code scanning of dosimeters to track movement of dosimeters to or from clients, and processing. Measuring and Test Equipment (M\&TE) is classified as "quality affecting” or "non-quality affecting.” Quality affecting M\&TE can directly influence the quality of the reported dose. Qualityaffecting M\&TE is calibrated and records kept in a manner that shows traceability to recognized national or international standards organizations.

The Hanford dosimetry system was procured based on HEDP design specifications. These design specifications were based on the recognized need for dosimeter capabilities to handle the broad spectrum of potential exposure environments in Hanford facilities. Hanford radiation fields have the potential for low-energy beta radiation from unsealed radiation sources, x-ray and gamma energies over a broad energy range, and neutron radiation. Prior to procurement, the design specifications were reviewed by the HPDAC with representation from all Hanford contractor dosimetry organizations at the time of the procurement to ensure that the system would be adequate for current and anticipated future field conditions.

\subsection{HEDP Documentation}

The HEDP practices are described in several PNNL manuals. These practices are implemented to comply with applicable DOE requirements, response(s) to program and/or facility appraisals, the DOELAP assessments, and "Hanford Practices” adopted through the HPDAC. These manuals are reviewed every three years at a minimum. A description of HEDP manuals follows:

- Hanford External Dosimetry Program Procedures Manual (PNL-MA841) -This manual provides the administrative and technical procedures for the HEDP, including those describing the organization and administration of the program, change control, and technical procedures. This manual contains all procedures used in the HEDP conduct of personnel, area, environmental, and nuclear accident dosimetry.

- Hanford External Dosimetry Technical Basis Manual (PNL-MA-842) This manual provides the technical bases and rationale for the design and implementation of the personnel, area, environmental, and nuclear accident dosimetry systems used at Hanford.

- Hanford External Dosimetry Program Data Management Manual (PNLMA-844) - This manual documents the design of the External Dosimetry Data Management System, a collection of programs used to process and manage dosimetry data. These programs were developed at PNNL specifically to support the Hanford dosimetry system and are not a commercial off-the-shelf product. In addition to screening and analysis of data from TLD readers, the programs calculate dose, implement 
process QC, import data from the Radiological Exposure System (REX), track dosimeters through the issue cycle to ensure all dosimeters are returned, processed, and results are reported. In addition to documenting the design and function of the software, PNL-MA-844 also documents hardware architecture, operating system, configuration control practices and data security practices.

- Radiation and Health Technology (R\&HT) Administrative Processes (PNL-MA-870) - This manual documents document control, document review and change control, variance reporting, contract review, internal assessment and management review, backup and maintenance of electronic records, and other practices that are common to all programs within R\&HT.

- $\quad$ R\&HT Quality Assurance Program Plan-This plan includes the basic quality assurance elements that are used by the HEDP staff when performing external dosimetry functions. The requirements contained in it were established and implemented in conformance with 10 CFR 830, Energy/Nuclear Safety Management, Subpart A, Quality Assurance Requirements (DOE 2003), DOE Order 414.1C Quality Assurance (DOE 2005a) and 10 CFR 835 Occupational Radiation Protection (DOE 2007a).

\subsection{HEDP Functional Relationship with DOE and DOE Contractors}

In addition to consideration of regulatory drivers, HEDP practices are formulated based on direct input from DOE and DOE Contractors. The primary mechanisms for external oversight and direction of HEDP are through the Hanford Personnel Dosimetry Advisory Committee (HPDAC), the Hanford Radiological Control Forum (HRCF), DOE Programmatic Assessments of HEDP and Contractor RPPs, and work agreements with each contractor and the associated SOWs.

\subsubsection{Hanford Personnel Dosimetry Advisory Committee}

The HPDAC was established by DOE-RL to provide technical guidance and establish uniformity in the administration of dosimetry programs at Hanford. The HPDAC is chaired by an RL representative, with identified representatives from other DOE site offices and respective contractor dosimetry organizations. The HEDP participates in HPDAC. Minutes of the monthly meetings are recorded and maintained in Hanford's radiological records historical file maintained by the Hanford Radiological Records Program (HRRP). HEDP technical issues and practices are coordinated through the HPDAC.

\subsubsection{Hanford Radiological Control Forum}

The Hanford Radiological Control Forum consists of representatives of the Hanford Site prime contractor's radiological control organizations and representatives of the RL/ORP radiological control organization. The chairperson is selected on a rotating basis. The activities of the HRCF include, but are not limited to: 
- review of radiological control consistency

- review of Hanford radiological problems and successes

- review of DOE radiological control guidelines.

Policy affecting issues developed through the HPDAC are occasionally presented to the HRCF for formal adoption. The HRCF is primarily a policy-setting organization, with one of its objectives to provide direction to all Hanford contractor organizations.

\subsubsection{Hanford Contractor Work Agreements}

The HEDP funding is provided by the respective Hanford contractor organizations. Letters of instruction and/or memoranda of understanding with associated SOWs are used to detail responsibilities, authority, and communication requirements of the respective organizations. Copies of these agreements are maintained in the HEDP Program files located in the 318 Building. In general, it is HEDP practice to incorporate primary responsibilities within the program objectives.

\subsubsection{Non-Hanford Contractor Work Agreements}

Non-Hanford contractors are responsible for establishing and documenting their practices for assigning, issuing, wearing, storing, and exchanging dosimeters. Generally, these practices are at the discretion of the customer, except where practices are constrained by the design of the dosimeter or by the limits of the accreditation. However, proper interpretation of the information stored in dosimeters requires that the processor have some knowledge of the radiation fields to which dosimeters are exposed and how dosimeters were worn, stored, and shipped. This information is conveyed in the SOW with the customer, through codes in files sent to HEDP at the start of processing of each batch, or through personal communication between customer and HEDP technical staff. The latter communication is kept in HEDP Program files.

\subsubsection{DOE Programmatic Assessments and Oversight}

HEDP is periodically assessed either directly or indirectly by DOE as part of DOE's programmatic assessments which include assessments of Hanford contractor Radiological Protection Programs. HEDP policies and practices are often shaped in response to these assessments.

\subsection{Field Dosimetry Practices and Contractor Responsibilities}

Each of the Hanford contractor organizations has radiation protection professionals who are responsible for field dosimetry practices and ultimately are responsible for approving the dose assigned to their personnel. Contractor responsibilities include the following:

- assignment of dosimeters to individuals through REX 
- issuing multiple dosimetry when necessary

- distribution, collection and accountability of dosimeters

- comparison of TLD and direct reading dosimeter (DRD) results and resolution of discrepancies

- review of dose results assigned to individuals

- implementing work restrictions as needed

- $\quad$ assessing dose for individuals with lost or damaged dosimeters

- determining which individuals are to be monitored with dosimetry

- determining proper exchange frequencies for routinely monitored employees

- $\quad$ enforcing proper dosimetry wear practices

- establishing proper controls on dosimetry storage and handling in the field

- assessment of field conditions to identify potential need for special algorithms and/or correction factors (See Chapter 6, Table 6.1)

- $\quad$ specifying appropriate facility calibration code when returning ring dosimeters to ensure field specific correction factors are applied when appropriate

- $\quad$ specifying appropriate facility calibration code when returning dosimeters to ensure application of proper neutron dose algorithm

- notification of HEDP when improper dosimeter response is suspected

- notification of HEDP when dosimeters are used outside the conditions for which they have been calibrated

- requesting technical support when appropriate

- assessment of equivalent dose to the skin from contamination

- assessment of effective dose from non-uniform radiation fields

- $\quad$ occurrence reporting

- administering workplace and area monitoring programs

- establishing locations for fixed nuclear accident dosimetry and exchanging of dosimeters 
- $\quad$ issue and assignment of personal nuclear accident dosimetry

- implementation of quick sort methods in the event of a criticality

Any number of the above tasks may be subcontracted to HEDP or other entities through detailed SOWs. 


\subsection{Quality Assurance and Quality Control}

HEDP quality assurance (QA) and quality control (QC) practices are described in R\&HT Quality Assurance Program Plan (R\&HT QAPP), PNL-MA-870 R\&HT Administrative Processes, and in PNL-MA-841, Hanford External Dosimetry Program Procedures Manual. The R\&HT QAPP contains specific quality assurance elements that ensure compliance with 10 CFR 830, Energy/Nuclear Safety Management, Subpart A, Quality Assurance Requirements (DOE 2003), DOE Order 414.1C, Quality Assurance (DOE 2005a), and 10 CFR 835, Occupational Radiation Protection (DOE 2007a). The objective of a quality assurance program is to ensure that equipment, software, and processes, perform as planned. This chapter focuses primarily on quality control and describes selected HEDP QC/QA implementing practices.

The principle objective of the HEDP is to provide high-quality dose measurement for Hanford workers, visitors, and the environment (Baumgartner, Endres, and Reese 1992). To attain this objective, the HEDP must

- maintain an effective, ongoing program to measure and verify process QC and dosimeter performance under controlled conditions and in the workplace

- recognize and promptly correct any factors that adversely affect quality

- maintain complete records of processing activities, program performance, and final reports and analyses to verify resulting data

- $\quad$ ensure that programmatic assessments and audits of HEDP (internal and external) are performed on a regular basis and identified deficiencies corrected

\subsection{Dosimetry Materials}

The HEDP staff examines all materials used in dosimeters. Because personnel and/or visitors are typically assigned only one dosimeter at a time, failure of any component of the dosimeter has the potential to adversely impact the quality of the recorded dose for the monitoring period in question. Therefore, HEDP conducts $100 \%$ acceptance testing of dosimeter cards and holders (every unit is tested prior to use) rather than random sampling of production lots. Acceptance testing of dosimeter cards involves tests of each element for:

- $\quad$ beta-photon sensitivity

- neutron sensitivity

- unwanted sensitivity to light

- glow curve structure

- reproducibility 
and tests of each card for:

- mechanical integrity

- bar code readability and uniqueness

- $\quad$ proper color coding

- proper phosphor placement

Acceptance testing of holders involves tests of:

- Proper composition of each filter

- Proper thickness of each filter

- Opaqueness to light for Mylar ${ }^{\circledR}$ beta window

- Adequate tinting of rose colored bar code window

- Integrity of gaskets and hinges

Acceptance testing of disposable components such as plastic ring casings, and Mylar windows is conducted on a production lot basis using sampling criteria from ANSI/ASQC Z1.4-1993 Sampling Procedures and Tables for Inspection by Attributes. Testing consists of 1) visual inspection for physical defects, 2) measurement of window thickness to verify thickness is within tolerance.

Each whole body dosimeter card and holder is uniquely labeled so that the entire history of dosimeter assignment and calculated dose can be tracked throughout their lifetime. Each reader system has a unique identifier that becomes a part of the permanent processing record. These unique, permanent identification numbers provide the capability to retroactively evaluate the quality of reported dose.

\subsection{Dosimetry Laboratories}

The HEDP dosimetry laboratory is designed and equipped to the extent practicable to minimize uncertainties in the TLD measurement process. Dosimetry laboratories and equipment are dedicated to dosimetry purposes only. To reduce electronic noise and to prevent loss of data from power failure, dedicated electrical circuits, with an installed UPS system, are used to power TLD reader systems. The laboratory spaces used by HEDP are temperature controlled and continuously monitored for temperature and humidity. Radiation levels are monitored monthly continuously with an area radiation monitor and assessed monthly using area monitoring TLDs. Radiation and contamination levels are checked monthly using hand held survey instruments as part of a routine radiological survey. Incoming dosimeters are surveyed for contamination in a separate room using an automated contamination survey table. Laboratory 
lighting is provided by UV filtered warm white fluorescent tubes with low emissions in the blue and ultraviolet regions so as to reduce spurious light induced signals on TL elements. Nitrogen gas used by the TLD reader systems is obtained from a liquid nitrogen source to ensure a low level of impurities such as moisture. Instrument quality air is provided for pneumatic devices and compressed inert gas is used for cleaning of TLD cards, and holders. HEPA filtered vacuum systems are used for cleaning of TLD readers and other laboratory equipment so as to minimize unnecessary spread of dust and chemical contaminants. Laboratory measurement and test equipment (M\&TE) used in quality affecting processes is controlled, calibrated and maintained as part of an ongoing M\&TE program.

Radiochemical analysis of NAD and PNAD components, personal items, and biological samples collected after a criticality accident are performed by the PNNL Analytical Chemistry Laboratory. The laboratory is located in the 325 Building in the 300 area.

\subsection{Dosimetry Processing Equipment}

HEDP dosimetry handling and processing equipment includes the following:

- Harshaw 5500, 6600, and 8800 TLD reader systems and associated computers and software

- TLD annealing ovens

- Automated holder openers

- Dosimeter contamination survey table

- Ultrasonic welder for sealing of rings

- Bar code scanning stations

- Label printers

\subsection{Design Features of TLD Reader Systems}

The heating system in all HEDP TLD reader systems consists, in principle, of two parts: 1) a heat-supplying medium, and 2) electronics for the control of temperature. The Harshaw Model 8800 and Model 6600 card readers use independent temperature-controlled streams of hot nitrogen gas to heat the individual TLD elements. Heating with nitrogen gas has been shown to have several advantages: the heat transfer from the gas to the dosimeter card is efficient and results in rapid readout, incandescent light emission is greatly reduced, and oxygen-induced background signals are easily suppressed. While these advantages are most pronounced for instruments in which the individual TLD phosphors are removed from their holder, the HEDP system has the following additional benefits even though the individual phosphors remain encased in cards during the heating cycle: 
- increased number of reuses without significant degradation in performance

- improved reproducibility of glow curve shape and integral TL signal

- $\quad$ simplified maintenance (Harshaw 1988)

The light-measuring equipment in the Harshaw TLD readers consist of a photomultiplier tube (PMT) in which light is converted into an electrical charge, an amplifier for this electric signal, and a signal registration unit that quantifies and stores the signal. In the Model 8800, four $1.27 \mathrm{~cm}$ diameter PMTs in a thermoelectrically cooled housing independently convert the emitted light from each of the four TLD elements in a card to electrical signals that are transmitted to the data acquisition system. In the Model 6600, two $1.27 \mathrm{~cm}$ diameter PMTs in a thermoelectrically cooled housing are used. In the Model 5500, one $1.27 \mathrm{~cm}$ diameter PMT in a thermoelectrically cooled housing is used.

The mechanical portion of the Model 8800 TLD reader consists of two carousels for holding cartridges of dosimeter cards, a readout station with PMTs, and a transport mechanism. In addition, the system is equipped with an internal ${ }^{14} \mathrm{C}$ light source for use in monitoring changes in the PMT sensitivity. The Model 6600 TLD reader has no carousels and accommodates only one load and one unload cartridge. The Model 8800 is capable of reading 1400 cards with a single loading. The Model 6600 is capable of reading 200 cards with a single loading. Details of reader design are contained in the manufacturer's literature kept in HEDP files.

The following performance characteristics have been adapted from manufacturer specifications for the 8800 and 6600 reader systems (Harshaw 1988):

- Electrical linearity: The electrical linearity of the system is $\pm 2 \%$, or \pm 2 $\mathrm{mR}$, whichever is greater, in the range from $1 \mathrm{mR}$ to $2000 \mathrm{R}$.

- Linearity with exposure: The TLD response is linear with exposure in the range of 0.001 to $100 \mathrm{R}$ to within $5 \%$.

- Dark current randomness: The standard deviation from the mean of 10 readings taken without heating and without a dosimeter in the instrument is less than $1 \mathrm{mR}$.

- System zero randomness: The standard deviation from the mean of ten readings taken with heating and with an unexposed dosimeter in the instrument is less than $5 \mathrm{mR}$.

- Residual reading: The readings from dosimeters, that have been initially irradiated to $500 \mathrm{mR}$, read, and then re-read without any annealing, are less than $2 \mathrm{mR}$.

- Reproducibility of heating: The reproducibility of the heating assembly is such that the same percentage of the total signal is extracted during the 
readout to within $\pm 1 \%$.

- Background stability: Over time intervals from one read-cycle up to 8 hours, the reader background (dark current) is reproducible to within $\pm 10 \%$ of a given threshold signal.

- $\quad$ Reproducibility of reader: Over a period of 8 hours, the readout value does not vary by more than $0.05 \%$ at a readout value corresponding to 100 times a given threshold signal.

- Card identification number: In conjunction with dosimeter assemblies, there are fewer than 1 in 10,000 erroneous dosimeter identifications, including dosimeter type and serial number. Each card can endure 500 readout cycles without any decrease in its mechanical performance, including dosimeter type and serial number identification/reading.

\subsection{Dosimetry Issuance and Receipt}

Daily activities within HEDP are directed toward processing dosimeters and interpreting results. However, other activities that precede and follow processing on the readers are necessary to ensure the quality of results. Process QC begins with preparing dosimeters for issuance. Once the dosimeter cards have been reader-annealed and oven-annealed and have been loaded into holders, the dosimeter (card and holder) barcodes are scanned to record the issued card/holder configuration. If the card/holder pairing is different upon return to the laboratory, an error message is generated when the dosimeter is scanned into the processing laboratory. When scanning the dosimeter for issue, the scanning software checks the status of several parameters within the HEDP database to establish eligibility of the dosimeter holder and card for issuance. The following are examples of conditions that will prevent the issuance of a dosimeter:

- The card has not passed one or more acceptance tests.

- The card has an invalid status (e.g. broken, lost, issued, or any status other than "annealed for issue.")

- The card anneal date is more than 30 days old.

- $\quad$ The card is due for recalibration.

- The holder has not passed acceptance testing.

- The holder has an invalid status (e.g. broken, lost, issued, or any status other than "returned").

- The card type and holder type are incompatible.

Dosimeters are scanned upon receipt for processing. The scanning software compares the issued configuration with the returned configuration and notifies the operator if changes have occurred. If the scanning transaction is successful, 
the dosimeters are inspected for damage and opened to remove the cards for processing.

\subsection{Dosimetry Processing}

Before processing TLD cards, an electronic QC check is performed on the TLD reader to be used. This check assesses several operating parameters, such as the mean and standard deviation of PMT noise (dark current), reference light (REF) readings, PMT high voltage, digital-to-analog converter voltage, 5- and 15-volt power supply voltages, and gas jet temperatures. The results are compared with user-determined limits and, if not acceptable, flagged for user evaluation.

Additionally, on a weekly basis, either a reader calibration or a reader functional check is performed. This check involves processing several cards exposed to $500-\mathrm{mR}$ radiation and verifies the function of the heating mechanism and the accuracy of the reader calibration factors to be applied.

After successful completion of the daily electronics check and weekly calibration or functional check, TLD cards may be read. Field cards are stacked into reader cartridges with two QC cards and two blank cards inserted at the beginning and a single QC and blank inserted after every 50 field cards. During readout, the reader applies real-time process QC by continuously monitoring the QC card readings, blank card readings, reference light readings, and PMT noise readings. If any single reading falls outside user prescribed limits, the reader stops processing. Typically, the prescribed limits are as follows:

- QC card readings - 450 to $550 \mathrm{mR}$

- blank card readings - 0 to $20 \mathrm{mR}$

- $\quad$ PMT noise limit - $5 \mathrm{mR}$ equivalent.

Reference light limits are set at approximately $\pm 10 \%$ of their nominal (long-term mean) value. If the reader stops, a number of evaluations are required by procedure before the reader can be put back into use for dosimetric readout of dosimeters. The reader also monitors the frequency of QC and blank card readings. If a QC and/or blank card is not processed within the user-prescribed number of field card readings, the reader stops. The PMT and reference light readings are automatically conducted by the reader at the user-prescribed frequency. During the read process, parameters such as gas pressure, gas temperature, and the PMT cooler temperature are continuously monitored. If they exceed the internal limits of the reader, they cause an orderly shutdown of the system. Finally, if a card cannot be successfully identified by the reader's barcode scanner, if there is no valid element correction coefficient (ECC) on file, or if the time temperature profile (TTP) to be applied by the reader during processing does not have a valid calibration, the card is rejected without being processed. 


\subsection{Data Screening}

The TLD cards are read in groups and the resulting electronic files produced by the reader are called group files. These group files are uploaded to the External Dosimetry Data Management System for consolidation with other group files and for data analysis. The External Dosimetry Data Management System resides on an Alpha computer system maintained by HEDP. After reading the data into temporary files, the data are screened to ensure that they were acquired under the proper reader configuration. In addition to the card IDs and raw element readings, each group file contains information regarding the TTPs and reader calibration factors (RCFs) applied during readout, the type of card being processed (e.g., field, QC, blank, calibration), the reading type (e.g., field reading, annealing, card calibration, etc.), the reader number and reader environment, data acquisition setup profile, and other information, which is compared with the configuration prescribed in HEDP procedures. The data are also compared with other data in the HEDP database for consistency. Cards not in the HEDP database are flagged. Cards showing no record of being returned to the HEDP processing laboratory are also flagged. The following are examples of the types of data checks performed by HEDP QC software:

- invalid reader number

- $\quad$ invalid group number

- invalid reader environment descriptor

- group file already imported

- dosimeter type inconsistent with reader environment

- External Dosimetry database key violation (record already in database based on reader number and date and time of reading)

- missing temperature information

- missing glow information

- duplicate card identification ID number in same group

- $\quad$ card not in database

- invalid reading date/time (i.e. subsequent to current system date/time, or of impossible value)

- TTP number inconsistent with reading type

- calibration option inconsistent with reading type

- $\quad$ invalid ECC (outside allowed range) 
- invalid RCF (outside allowed range)

- invalid instrument type

- group number in record inconsistent with user input

- REMS environment in record inconsistent with user input

- $\quad$ card ID number invalid for REMS environment used

- card ID number inconsistent with dosimeter type used

- reading type inconsistent with group purpose

- card ID number inconsistent with reading type

If any record in the group file fails the above data-screening parameters, the group file is flagged for evaluation. In either case, a data screen report is generated to provide a summary of error conditions.

\subsection{Group QC Statistics}

After the data in the group file are incorporated into the HEDP database system, the PMT, reference light, QC card and blank card readings, and other QC-related information in the group are analyzed. A group QC statistics report is generated, summarizing the analysis. This report provides timely feedback on the stability of the reader and the acceptability of the process QC implemented at the reader level. By calculating the mean, and standard deviation of PMT dark current, reference light, and QC and blank dosimeter readings in each group file, a tighter level of QC can be maintained on dosimeter processing. Limits are established at the External Dosimetry database level for the minimum number of QC readings of each type (i.e., PMT dark current, reference light, QC, and blank readings) that must be contained in each group file, as well as limits for the minimum, maximum, mean and standard deviation for each of these reading types.

Typically, the limits for the QC card mean in each group is $\pm 5 \%$ of the delivered exposure. The limits for the other reading means are $\pm 5 \%$ around their nominal values, as well. If all of the group file statistics are within an acceptable range, the group file is judged to have acceptable QC. A database record is kept of the QC statistics associated with each group file. If any QC result in a group file does not have acceptable QC statistics, each dose result record generated from the group file is flagged for evaluation and prevented from being reported until resolution. 


\subsection{Glow Curve Analysis}

Glow curves for each chip on each card are analyzed for acceptable peak centroid and full-width at half-maximum and for the presence of single-channel spikes that contribute significantly to the total glow integral. Four regions of interest in each glow curve are analyzed. If the glow curve data do not conform to an expected pattern, the curve is flagged as having abnormal characteristics. Dose result records based on flagged glow curves are also flagged. Flagged dose records are evaluated by a dosimetrist to determine whether adjustments to data are necessary. Flagged dose records are prevented from being reported until data anomalies have been resolved.

\subsection{Element Ratio Analysis}

One of the first steps in the dose calculation process is the analysis of chip readings relative to each other. Three independent element ratios result from the four-chip dosimeter cards. If any one of these ratios is outside the preestablished range expected from the radiation types to which the dosimeter could have been exposed, the dosimeter record is flagged for evaluation. In addition to tests of element ratios, numerous other tests are performed on raw element readings, intermediate results and final results by the dose calculation software. If any of these tests are failed, an algorithm flag is set on the individual dose result record which prevents reporting of the dose result until the anomaly is investigated and resolved.

\subsection{Review and Reporting of Dose Results}

By procedure, every calculated dose result must be reviewed by a qualified dosimetrist before reporting. For those calculated dose results that have one or more software generated QC flags, the dosimetrist must clear the flag before dose results can be reported. To clear the flag, the dosimetrist must determine the cause of the flag, assess whether the result is accurate as is, or needs to be adjusted, make adjustments if necessary, and document the basis for the adjustments. In most cases, the adjustment is made to one or more raw chip readings on the basis of glow curve structure, and dose is recalculated using the adjusted readings. In addition to the individual results in a process group, the dosimetrist must verify that the process QC parameters associated with the group (e.g. group QC statistics) are within the allowed tolerances before dose results can be reported. Because dosimeters must be processed and dose results reported on a daily basis, process QC is designed such that a sufficient level of confidence in the accuracy of dose results can be achieved to allow reporting of dose results on a daily (i.e. on a group basis) rather than at the end of a predetermined time period (e.g. monthly). As such, reporting of dose results is not contingent upon availability of audit dosimeter results and the role of audit dosimeters is more closely related to quality assurance rather than quality control. The requirements for reporting of dose results are resolution of all flags, verification of adequate process QC, and dosimetrist review and signature on hardcopy dose calculation reports. 


\subsection{Dose Results Accountability}

For every dosimeter returned for processing, the HEDP must ensure that a dose result is reported, or in the case of damaged dosimeters, notify the customer that a dose result cannot be determined. For each dosimeter issued to a customer, the status of the card and holder is tracked in the External Dosimetry database from the time of issue through final reporting of dose results. This status tracking ensures that all issued dosimeters will be accounted for and that dose results will be reported in a timely manner for all dosimeters returned to the lab for processing. The following listings are routinely generated from the External Dosimetry database and acted upon by the HEDP staff:

- Dosimeters issued but not returned within allotted time frame (overdue)

- Dosimeters returned (according to the customer) but not scanned in (to the Lab) within allotted time frame

- Dosimeters scanned in but not processed within allotted time frame

- Dosimeters processed but not reported within allotted time frame

\subsection{Unreturned Dosimeters}

Dosimeters that have not been returned to the lab within the expected time frame for each exchange frequency will appear on an "over due" dosimeter report that is used for billing purposes. Monthly, quarterly, and annual dosimeters that have not been returned to the lab within 60, 180 and 465 days respectively of their scan out date will be listed. Dosimeters issued without an exchange frequency (e.g. temporary dosimeters) are treated as annual dosimeters for this purpose.

\subsection{Lost or Damaged Dosimeters}

In cases where a dosimeter has been lost or has been returned in a condition too damaged for processing, the client is expected to conduct an investigation to determine the dose to be assigned. For Hanford clients, the results of the investigation are documented on an Investigation of Dosimeter Result (IODR) form. The form is used by the HRRP staff to enter the estimated dose into the individual's exposure history file. Non-Hanford clients are responsible for review and input of the assessed dose into their own radiological records database. Hanford and Non-Hanford clients are notified by HEDP of dosimeters too damaged to be successfully processed and dosimeters over due for processing. 


\subsection{5 “High” Dose Results}

The HEDP system is capable of flagging dose results during dosimeter processing, that exceed agreed-upon dose levels requiring prompt notification of the client dosimetry organization. For Hanford clients, prompt notification is made when a dosimeter result contains a dose component exceeding one of the following:

Table 3.1. Prompt Notification Levels

\begin{tabular}{||l|l|r||}
\hline Dosimeter Type & \multicolumn{1}{|c|}{$\begin{array}{c}\text { Dose Equivalent } \\
\text { Quantity }\end{array}$} & $\begin{array}{c}\text { Notification } \\
\text { Level (mrem) }\end{array}$ \\
\hline \hline HSD, 8825BP & $\mathrm{H}_{\mathrm{p}}(0.07)$ & 2000 \\
\hline HSD, 8825BP & $\mathrm{H}_{\mathrm{p}}(3)$ & 1000 \\
\hline HSD, 8825BP & $\mathrm{H}_{\mathrm{p}}(10)_{\gamma}$ & 300 \\
\hline HSD, 8816 & $\mathrm{H}_{\mathrm{p}}(10)_{\eta}$ & 500 \\
\hline HSD & $\mathrm{H}_{\mathrm{p}}(10)_{\gamma}+\mathrm{H}_{\mathrm{p}}(10)_{\eta}$ & 3000 \\
\hline Ring & $\mathrm{H}_{\mathrm{p}}(0.07)$ & 500 \\
\hline
\end{tabular}

Note: The prompt notification levels for an HSD dosimeter worn on an extremity (e.g. HSD Wrist dosimeter) are the same as for an HSD worn as a whole body dosimeter.

These records are automatically flagged as "high" in the HEDP database system. Following review and approval by HEDP dosimetrists who apply a knowledge of the algorithms and processing equipment, these results are reported on a priority basis to the respective clients' dosimetry organizations. For Hanford clients, dose results are electronically reported to REX by 10:00 AM the morning following determination of dose and the contractor dosimetry representative is notified by e-mail or by phone at the same time. The dosimeter result records sent to REX are individually flagged in a manner that REX recognizes as a "high" dose. It should be noted that the notification levels above apply to individual dosimeters. The HCND is actually two dosimeters $(8825 \mathrm{BP}+8816)$ and the dose calculation/flagging software applies limits to their deep photon and neutron results independently.

\subsection{Abnormal Dosimeter Results - Note Codes}

Whenever a dosimeter result is suspected of being in error based on the raw glow or heat curve data, or dose algorithm flags, or detection of unexpected radiation types based on the work environment in which the dosimeter was used, HEDP notifies the client as described below. The results are identified with "note codes" of 50, 53, or 59 in the note code field of the dose results file provided for input to REX (or the equivalent radiological records database for non-Hanford customers). 
Note code 50 identifies results for which HEDP is unable to confidently estimate a dose based on observed processing data and for which the client is expected to perform an investigation to determine the dose to be recorded. Dosimeter results with a note code of 50 are generally reported as calculated from the raw data, without any attempt at correction or adjustment. When raw data cannot be obtained (e.g. badly damaged or contaminated dosimeter) the Note code 50 result is reported as 0 mrem. Note code 53 results represent results for which HEDP has revised one or more chip readings because of abnormalities in the glow curve or other raw processing data, then recalculated the dose. Note code 59 results are results that include a positive neutron dose when neutron dose is not expected based on the facility calibration code under which the dosimeter was submitted for processing. The default facility calibration code (00) is defined such that neutron dose $\underline{i s}$ expected. Facility calibration codes are discussed in greater detail in Chapter 5, Section 5.3.8. For Hanford clients, note code 53 and 59 dose results, as reported in the dose results file, are automatically entered into REX whereas note code 50 results are not. For note code 50 results, an investigation must be performed by the client to determine the correct dose to enter into REX. Practices for non-Hanford clients regarding incorporation of HEDP dose results into their radiological exposure records databases varies from client to client. Non-Hanford clients are instructed to treat each note code 50 result as a nonresult requiring investigation to determine dose in the same manner as a lost or damaged dosimeter that could not be processed.

For all note code 50 and 59 records and selected note code 53 records (see paragraph below), the HEDP database system automatically produces a "Suspect Dosimeter Results Evaluation Form.” This form is mailed to the respective Hanford client dosimetry representative for notification of the suspect result. For note code 53 and 59 results, the Hanford client provides a concurrence signature and returns the form to HRRP for inclusion in the individual's exposure history record. In rare cases, if the client does not agree with the reported dose they may elect to complete an IODR form assigning the dose to be recorded. For note code 50 results, the Hanford client must document the dose to be assigned on an IODR form. In either case, the IODR form is sent to the HRRP for inclusion in the individual's exposure history files and for use by HRRP staff members in updating REX. Similar arrangements are available to all users of HEDP services.

HEDP has latitude to make corrections to chip readings as appropriate to improve accuracy of reported dose and reduce false positive results. Such changes are typically based on clearly identified noise or other abnormalities in the glow curve and are made on a regular basis. Changes of this type are always identified with a note code of 53 in the electronic dose record used to update REX. However, "Suspect Dosimeter Results Evaluation Forms" are generated and forwarded to contractor dosimetry organizations for concurrence only when there is a change in calculated dose (increase or decrease) that exceeds established thresholds. The current thresholds requiring contractor notification and concurrence are shown in Table 3.2. These thresholds have been agreed to by the HPDAC and apply to all dosimeter types. 
Table 3.2 Change in Calculated Dose Requiring Contractor Concurrence

\begin{tabular}{|l|r|}
\hline \multicolumn{1}{|c|}{$\begin{array}{c}\text { Dose Equivalent } \\
\text { Quantity }\end{array}$} & \multicolumn{2}{c|}{$\begin{array}{c}\text { Change in } \\
\text { Calculated Dose } \\
\text { (mrem) }\end{array}$} \\
\hline \hline $\mathrm{H}_{\mathrm{p}}(0.07)$ & 1,500 \\
\hline $\mathrm{H}_{\mathrm{p}}(3)$ & 450 \\
\hline $\mathrm{H}_{\mathrm{p}}(10)_{\gamma}$ & 50 \\
\hline $\mathrm{H}_{\mathrm{p}}(10)_{\eta}$ & 50 \\
\hline
\end{tabular}

\subsection{Blind Audit Dosimeter Program}

In addition to the above QC methods, the HEDP maintains an ongoing blind audit dosimeter program as a quality assurance measure. This is an in-house (R\&HT) blind test of HEDP. Each month, the R\&HT Quality Manager arranges for exposure of blind audit dosimeters to radiation sources and doses unknown to HEDP dosimetrists or processing staff. The dosimeters used as audit dosimeters are labeled for and assigned to fake individuals in REX and are indistinguishable to HEDP staff members from other assigned dosimeters. On a quarterly basis, audit dosimeter results are evaluated against the delivered doses and performance is determined according to the methodology in the DOELAP performance test standard (DOE 1986a). The results of these performance evaluations are reported to DOE-RL, Hanford contractor dosimetry organizations and are retained in HEDP records.

\subsection{Dosimetry Processing Quality Assurance Reports}

Quality assurance of dose results is provided by the HEDP on a quarterly basis by plotting and statistical evaluation of PMT readings, REF readings, QC card readings, and Blank card readings, as well as analysis of monthly blind audit dosimeter performance against DOELAP criteria. On an annual basis, PMT readings, REF readings, QC card readings, and Blank card readings and RCFs are plotted and statistically evaluated over a period representing a calendar year. The performance of quarterly and annual blind audit dosimeters is also evaluated against DOELAP criteria. The results of all these analyses are documented in HEDP files in dosimeter processing quality assurance reports generated by a HEDP Dosimetrist.

\subsection{External Dosimetry Program Records}

The HEDP maintains comprehensive records of all processing data, including the following:

- $\quad$ electronic records of all processing data 
- $\quad$ electronic records of all QC data to include qualification testing of dosimeter cards and holders

- $\quad$ electronic records of the digitized glow curves

- $\quad$ hard-copy records of dose evaluation for abnormal circumstances

- $\quad$ electronic records of the use history of all HEDP personnel dosimeter cards and holders

- $\quad$ hard-copy records of procedures

- $\quad$ hard-copy records of QA requirements

- $\quad$ hard-copy records of letters of instruction, memoranda of understanding, or contracts

- $\quad$ hard-copy records of training and staff qualifications

- $\quad$ hard-copy records of DOELAP and NVLAP certificates and letters of instruction, accreditation status, responses to DOELAP onsite assessments

- $\quad$ electronic records to retroactively evaluate dose

- $\quad$ hard-copy records of technical studies or documentation.

The foregoing records are an important element of the HEDP. They provide the detailed information necessary to technically validate the calculation of reported dose, the capability to retroactively evaluate dose upon request, and the information needed to meet regulatory, technical and QA requirements.

\subsection{Records Disposition}

DOE has prescribed requirements for the orderly disposition of records at DOE facilities. External dosimetry records are considered to be under the general heading of medical, health, and safety records. The requirements for records disposition separate dosimetry information into program records and individual exposure records. Program record requirements include the following four categories:

- Results of equipment calibration establishing the authenticity of the dose results must be held for 75 years.

- Automatic data processing system programs, codes, instructions, tapes, and discs, if used for the retrieval of dosimetry data, must be held for 75 years.

- Worksheets, requests for analysis, charts containing information that must be interpreted or further modified before use, automatic data 
processing system input records, information used in interim calculations, and information used to verify that the recorded data are correct, must be held until the exposure record has been verified and approved or for a period of 1 year, whichever is earlier.

- Historical files of standards, guides, and procedures (including revisions) must be retained permanently.

Individual exposure records require a 75-year retention period for the following types of information: records of contamination incidents, results of dose assessments, and documentation on any investigations undertaken.

\subsection{Other Quality Assurance Topics}

The following QA topics are described in greater detail in the $R \& H T Q A P P$, PNL-MA-870 R\&HT Administrative Processes, and PNL-MA-841 Hanford External Dosimetry Procedures Manual:

- $\quad$ R\&HT Quality Policy

- R\&HT Organization and Personnel

- Personnel Training and Qualification

- Document Control

- Review of Work Requests

- Subcontracting

- Purchasing of Services and Supplies

- Reviewing Client Service Requirements

- Handling of Client Complaints

- Control of Non-Conforming Work or Items

- Variance Reporting

- Change Control

- Records Control

- Internal and External Assessments

- Management Reviews

- Confidentiality and Proprietary Rights

- Preventive Action

- Corrective Action

- Action Tracking 
THIS PAGE INTENTIONALLY LEFT BLANK 


\subsection{Regulatory Basis}

The primary DOE requirements for occupational radiation protection are provided in 10 CFR 835 (DOE 2007a). This rule codifies DOE radiation protection requirements that are based on recommendations of the International Commission on Radiological Protection (ICRP) in ICRP Publication 60 (ICRP 1991). In addition, DOE has issued a number of guidance documents. HEDP implements DOE requirements and guidance as directed in statements of work (SOWs) with DOE and Hanford Contractors. The HEDP also implements specific clarifications, agreements or directives identified through the HPDAC that do not conflict with regulatory requirements or contractor SOWs. Basic DOE requirements and guidance pertaining to external dosimetry are identified in this chapter. The means by which dosimeter results (expressed as operational quantities) are used to demonstrate compliance with regulatory requirements (expressed as protection quantities) is also discussed.

\subsection{DOE Requirements}

DOE requirements applicable to the HEDP are defined in the following documents:

- 10 CFR 835 Occupational Radiation Protection (DOE 2007a)

- 10 CFR 830 Energy/Nuclear Safety Management, Subpart A, Quality Assurance Requirements (DOE 2003)

- $\quad$ DOE Order 414.1C Quality Assurance (DOE 2005a)

- DOE-STD-1095-95 Department of Energy Laboratory Accreditation Program for Personnel Dosimetry Systems (DOE 1995)

- DOE-STD-1111-98 The Department of Energy Laboratory Accreditation Program Administration (DOE 1998a)

- DOE/EH-0027 Department of Energy Standard for the Performance Testing of Personnel Dosimetry Systems (DOE 1986a)

- DOE/EH-0026 Handbook for the Department of Energy Accreditation Program for Personnel Dosimetry Systems (DOE 1986b)

- $\quad$ DOE O 231.1A, Chg. 1. Environment, Safety and Health Reporting (DOE 2004)

- $\quad$ DOE M 231.1-1A, Chg. 2, Environment, Safety and Health Reporting Manual. (DOE 2007b)

- Hanford Radiological Health and Safety Document (DOE 2001) 
These requirements are applicable to Hanford contractors using the services of HEDP, to the extent that they are referenced and stipulated in their individual contracts with DOE.

\subsection{DOE Guidance}

DOE technical guidance applicable to the HEDP is provided in the following documents:

- $\quad$ DOE G 441.1-1C Radiation Protection Programs Guide (DOE 2008a)

- DOE-STD-1098-2008 Radiological Control (DOE 2008b)

- RCTP 2009-01,Technical Position Clarifying Dose Assessment and Recording (DOE 2009)

Chapters 6 and 8 and 13 of DOE G 441.1-1C Radiation Protection Programs Guide contain several provisions which HEDP has adopted as standard practice. DOE guidance implemented by HEDP includes the following:

- maintenance of a technical basis manual providing scientific information and other rationale explaining each element of the external dosimetry program

- maintenance of historical records of personnel dosimeter measurement results and dose assessments

- conduct of an internal audit program no less frequently than every three years

- personnel dosimeter measurement methods and frequencies appropriate for the specific dosimetry applications at Hanford.

- methods for control, accountability, and safe handling of dosimeters

- $\quad$ appropriate action level and investigation level guidelines

- $\quad$ historical records of the external dosimetry program and procedures, as well as changes in the programs and procedures

- QA program covering all aspects of activities that determine worker dose

- methods for the use of multiple dosimetry and a defined methodology for dose calculation

- $\quad$ performance criteria for nuclear accident dosimetry, to include a system of fixed and personal dosimeters, documentation of placement criteria, and analytical methods

- methods for assessment of dose to extremities or skin from non-uniform irradiation 
- methods for evaluating the various doses from external radiation

\subsection{DOE Laboratory Accreditation Program}

10 CFR 835 requires the accreditation of dosimetry programs. Four documents describe the DOE program for accrediting dosimetry programs at DOE facilities:

1. The Department of Energy Laboratory Accreditation Program Administration DOE-STD-1111-98 (DOE 1998a) describes overall administration of the program.

2. Standard for the Performance Testing of Personnel Dosimetry Systems DOE/EH-0027 (DOE 1986a) contains performance criteria for dosimetry systems similar to that of HPS N 13.11 (HPS 2009).

3. Handbook for the Department of Energy Laboratory Accreditation Program for Personnel Dosimetry Systems DOE/EH-0026 (DOE 1986b), contains requirements for Personnel Training and Qualification, Materials and Equipment, Procedures, Quality Assurance, Documentation, and Dose Reporting.

4. Department of Energy Laboratory Accreditation Program for Personnel Dosimetry Systems DOE-STD-1095-95 (DOE 1995) reestablishes the source of technical criteria used in the accreditation of personnel dosimetry systems to be DOE/EH-0026 and DOE/EH-0027 (as originally specified in DOE Order 5480.11 before its replacement with 10 CFR 835).

DOELAP evaluates dosimeter performance based on the dosimeter's response to known operational quantities. The standards establish acceptable performance in multiple test categories, described generally as follows:

- accident level test categories for photons extending from 10 to $500 \mathrm{rad}$

- protection level test categories for photon, beta, and neutron radiation extending from 30 mrem to 10 rem.

- $\quad$ subcategories of testing for single sources and for mixtures (e.g., photon/beta, photon/neutron mixtures)

- Acceptable performance defined as precision + bias is less than $30 \%$ for single sources and $40 \%$ for mixtures.

These standards also established required type testing as follows:

- documentation of dosimeter angular response typically to include evaluations of angular response for exposure angles of $0^{\circ} \pm 40^{\circ}, \pm 60^{\circ}$, and $\pm 85^{\circ}$, for both vertical and horizontal planes of rotation 
- documentation of lower level of detection (LLD) for each protection dose level category

\subsection{Operational and Protection Quantities}

In the June 7, 2007 amendment to DOE's rulemaking 10 CFR 835 Occupational Radiation Protection (DOE 2007), DOE replaced ICRP 26 dosimetric concepts with ICRP 60 dosimetric concepts as the basis of its system of radiation protection and dose limitation. Two types of quantities have been defined for use in radiological protection: Protection quantities, which are defined by the ICRP, and operational quantities which are defined by the ICRU. The protection quantities recommended in ICRP Publication 60 (ICRP 1991) include the effective dose, $\boldsymbol{E}$, and the tissue or organ equivalent dose, $\boldsymbol{H}_{\mathrm{T}}$. These quantities are not directly measurable but can be calculated if the conditions of irradiation are known. The ICRU has defined a set of operational quantities for area and individual monitoring. These quantities were developed in response to the ICRP's recommendations in ICRP Publication 26 (ICRP, 1997). They were designed to be measurable, to provide a conservative estimate of the protection quantities in existence at that time (ICRU, 1985, 1993b) and to serve as calibration quantities for dosimeters used in monitoring. For area monitoring, the appropriate operational quantities are the ambient dose equivalent, $\boldsymbol{H}^{*}(\boldsymbol{d})$, and the directional dose equivalent, $\boldsymbol{H}^{\prime}(\boldsymbol{d}, \Omega)$. For individual monitoring, the personal dose equivalent, $\boldsymbol{H}_{\boldsymbol{p}}(\boldsymbol{d})$, is the appropriate operational quantity. These operational quantities are defined in the glossary of this manual. The ICRP and ICRU have together determined that for most exposure conditions, these operational quantities are still conservative with respect to the ICRP 60 protection quantities (ICRP 1966).

In the 2007 amendment to 10 CFR 835, the quantity equivalent dose replaced the previous quantity dose equivalent (to a tissue or organ) and the quantity effective dose replaced the previous quantity effective dose equivalent. These quantities may result from internal or external sources. When the dose is from internal sources, the terms committed equivalent dose and committed effective dose are typically used to indicate 50 year dose commitments. The new ICRP 60 quantities used by DOE now differ from the previous ICRP 26 quantities in two fundamental ways. 1) Equivalent dose is based on absorbed dose averaged over the mass within a volume of tissue (e.g. organ) whereas dose equivalent is based on the absorbed dose at a point in tissue. 2) Equivalent dose is determined by multiplying the average absorbed dose in a tissue volume by a radiation weighting factor whereas dose equivalent is determined by multiplying the absorbed dose at a point in tissue by a quality factor. Quality factors are a function of the quality of radiation (i.e. LET distribution) at the specified point in tissue and are influenced by tissues surrounding the point of interest in the body. Radiation weighting factors are a function of the quality of radiation incident upon the body and are not influenced by tissues surrounding the organ or tissue of interest in the body.

ICRP Publication 60 did not recommend changes in the definitions of operational quantities for personnel and area monitoring. However, it did recommend a change in the Q-LET relationship used to calculate the quality factors that are used in the calculation of those operational quantities. When quality factors for 
neutrons are calculated at a $1 \mathrm{~cm}$ depth in the ICRU tissue sphere using this relationship and plotted as a function of incident neutron energy, the values are very similar to the ICRP 60 radiation weighting factors plotted as function of energy (ICRP, 1991). The primary impact of the revision of quality factors and the adoption of new radiation weighting factors on external dosimetry is a significant increase in the values obtained for both operational quantities and protection quantities in neutron radiation fields. In ICRP Publication 74, the ICRP has shown that operational quantities, when calculated using quality factors based on the ICRP 60 Q-LET relationship, are still conservative estimators of the ICRP 60 protection quantities in nearly all cases (ICRP 1996).

\subsubsection{How Operational Quantities and Dose Assessment Results are Stored in REX}

For personnel monitoring, HEDP dosimeters measure and report the following operational quantities (personal dose equivalent):

$$
\begin{aligned}
& \mathrm{H}_{\mathrm{p}}(0.07)_{\beta \gamma} \\
& \mathrm{H}_{\mathrm{p}}(3)_{\beta \gamma} \\
& \mathrm{H}_{\mathrm{p}}(10)_{\gamma} \\
& \mathrm{H}_{\mathrm{p}}(10)_{\eta},
\end{aligned}
$$

Occasionally a second subscript ("wb” or "extr") is used to distinguish between results reported for whole body locations as apposed to extremity locations. Because HEDP dosimeters are capable of measuring and reporting only the betagamma component of $H_{p}(0.07)$ and $H_{p}(3)$, the $\beta \gamma$ subscript is generally understood and omitted when specifying HEDP dosimeter results. However, to ensure clarity, the subscript has been retained in the tables below. The dosimeter reported value for $\mathrm{H}_{\mathrm{p}}(10)_{\gamma}$ is the contribution to $\mathrm{H}_{\mathrm{p}}(10)$ from external betagamma radiation. The subscript indicating beta radiation is omitted because beta radiation is a negligible contributor to $\mathrm{H}_{\mathrm{p}}(10)$ at Hanford. To the extent that energetic beta may contribute to the deep dose equivalent, its contribution is included in the $\mathrm{H}_{\mathrm{p}}(10)_{\gamma}$ value. The dosimeter reported value for $\mathrm{H}_{\mathrm{p}}(10)_{\eta}$, is the contribution to $\mathrm{H}_{\mathrm{p}}(10)$ from external neutron radiation.

REX contains the following fields for storage of dose information that is summed to obtain protection quantities:

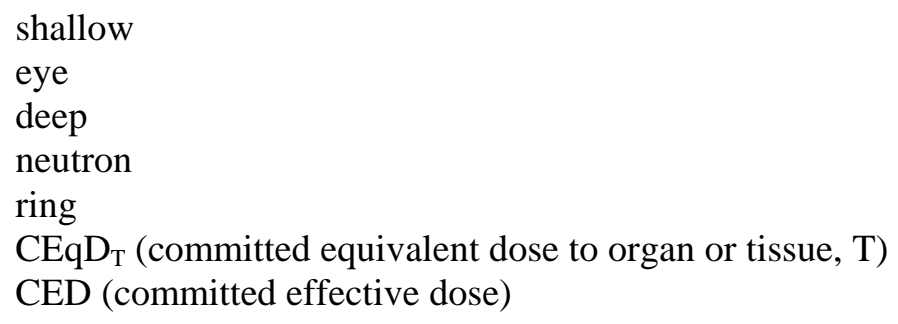

Table 4.1 shows how dosimeter results and dose assessment results are entered into these fields in REX. Multipack results are shown as protection quantities equivalent dose or effective dose rather than operational quantities, because they are in essence a dose assessment. Multiple dosimeter results are weighted to obtain a single reported value that is intended to estimate a protection quantity. Considerable latitude and professional judgment may be involved in the 
multipack dose assessment process. The result does not fit the definition of the operational quantity personal dose equivalent in that it does not reflect a measurement made at a specified point on the body. Similarly, the results of skin dose assessments using VARSKIN 3 are shown as protection quantities (equivalent dose). Although skin dose assessments involve calculation of dose equivalent at a specified depth, they also involve dose averaging over areas and weighting according to the methodology given 10 CFR 835.205. The result is an assessment of the protection quantity, equivalent dose to the skin. Results from the IODR process are shown as protection quantities for the same reasons. The process is used for assessing dose for unmonitored individuals or individuals who lost their dosimeter, as well as special circumstances where an assessment of dose from dosimeter results is required. For this reason the quantities shown in this column are shown as equivalent dose and effective dose. Assessment of dose from Multipacks is one example of the IODR process.

Table 4.1 Storage of Dosimeter Results and Dose Assessment Information in REX Fields

\begin{tabular}{|c|c|c|c|c|c|c|c|c|}
\hline \multirow{3}{*}{$\begin{array}{l}\text { REX } \\
\text { Data } \\
\text { Field }\end{array}$} & \multicolumn{8}{|c|}{ Dose Inputs to REX Field } \\
\hline & \multicolumn{4}{|c|}{ Routine Dosimeter Results } & \multicolumn{4}{|c|}{ Dose Assessments } \\
\hline & HSD & 8825 BP & 8816 & EXT-RAD ${ }^{(a)}$ & $\begin{array}{l}\text { Multi- } \\
\text { pack }^{(b)}\end{array}$ & VARSKIN $3^{(c)}$ & IODR & Bioassay \\
\hline shallow & $H_{p}(0.07)_{\beta \gamma, w b}$ & $H_{p}(0.07)_{\beta \gamma, w b}$ & & & $H_{\text {skin }}$ & $H_{\text {non-U-skin }}$ & $H_{\text {skin }}$ & \\
\hline eye & $H_{p}(3)_{\beta \gamma, w b}$ & $H_{p}(3)_{\beta \gamma, w b}$ & & & $H_{\text {eye }}$ & & $H_{e y e}$ & \\
\hline deep & $H_{p}(10)_{\gamma, w b}$ & $H_{p}(10)_{\gamma, w b}$ & & & $E_{\gamma}$ & & $E_{\gamma}$ & \\
\hline neutron & $H_{p}(10)_{\eta, w b}$ & & $H_{p}(10)_{\eta, w b}$ & & $E_{\eta}$ & & $E_{\eta}$ & \\
\hline ring & & & & $H_{p}(0.07)_{\beta \gamma, \text { extr }}$ & $H_{\text {extr }}$ & $H_{\text {non-U-extr }}$ & $H_{\text {extr }}$ & \\
\hline$C E q D_{T}{ }^{(d)}$ & & & & & & & & $\begin{array}{c}H_{T, 50} \\
H_{\text {emb/fetus, in utero }} \\
\end{array}$ \\
\hline CED ${ }^{(\mathrm{e})}$ & & & & & & & & $E_{50}$ \\
\hline
\end{tabular}

(a) For each pair of rings, REX takes the highest result as the "ring" dose.

(b) The protection quantities in this column are assessed from dosimeter reported operational quantities using the Multiple Dosimeter Methodology outlined in Chapter 6. $H_{\text {skin }}$ is based on the highest $\left[H_{p}(0.07)+H_{p}(10)_{\eta}\right]$ reported by a dosimeter worn in a whole body location. $H_{\text {eye }}$ is based on the highest $\left[H_{p}(3)+H_{p}(10)_{\eta}\right]$ reported by a dosimeter worn in a whole body location. $\mathrm{H}_{\text {extr }}$ is based on the highest $\left[\mathrm{H}_{\mathrm{p}}(0.07)+\mathrm{H}_{\mathrm{p}}(10)_{\eta}\right]$ reported by a dosimeter worn in an extremity location. The HSD Wrist/Ankle dosimeter is the only extremity dosimeter capable of measuring neutron dose. When used, it must be used as part of a multipack.

(c) The non-uniform equivalent dose for the skin and the extremity are assessed for areas $>10 \mathrm{~cm}^{2}$ using the methodology given in 10 CFR 835.202.

(d) $\mathrm{CEqD}_{\mathrm{T}}=$ committed equivalent dose to a specified organ or tissue $\mathrm{T}$. There is a $\mathrm{CEqD}_{\mathrm{T}}$ field for each tissue. In the case where $\mathrm{T}=$ embryo/fetus, the actual quantity to be entered is currently under review, awaiting clarification from DOE.

(e) $\mathrm{CED}=$ committed effective dose 


\subsubsection{Relevant 10 CFR 835 Requirements}

The relevant 10 CFR 835 requirements where protection quantities are used are excerpted below. The protection quantity is shown in bold.

§ 835.202 Occupational dose limits for general employees.

(a) Except for planned special exposures conducted consistent with § 835.204 and emergency exposures authorized in accordance with § 835.1302, the occupational dose received by general employees shall be controlled such that the following limits are not exceeded in a year:

(1) A total effective dose of 5 rems (0.05 Sv);

(2) The sum of the equivalent dose to the whole body for external exposures and the committed equivalent dose to any organ or tissue other than the skin or the lens of the eye of 50 rems $(0.5 \mathrm{~Sv})$;

(3) An equivalent dose to the lens of the eye of $15 \mathrm{rems}(0.15 \mathrm{~Sv})$; and

(4) The sum of the equivalent dose to the skin or to any extremity for external exposures and the committed equivalent dose to the skin or to any extremity of 50 rems $(0.5 \mathrm{~Sv})$.

$\S 835.206$ Limits for the embryo/fetus.

(a) The equivalent dose limit for the embryo/fetus from the period of conception to birth, as a result of occupational exposure of a declared pregnant worker, is 0.5 rem $(0.005 \mathrm{~Sv})$.

$\S 835.207$ Occupational dose limits for minors.

The dose limits for minors occupationally exposed to radiation and/or radioactive materials at a DOE activity are 0.1 rem $(0.001 \mathrm{~Sv})$ total effective dose in a year and 10 percent of the occupational dose limits specified at $\S$ 835.202(a)(3) and (a)(4).

$\S 835.208$ Limits for members of the public entering a controlled area.

The total effective dose limit for members of the public exposed to radiation and/or radioactive material during access to a controlled area is 0.1 rem $(0.001$ Sv) in a year.

§ 835.702 Individual monitoring records.

(c) The records required by this section shall:

(1) Be sufficient to evaluate compliance with subpart $\mathrm{C}$ of this part;

(2) Be sufficient to provide dose information necessary to complete reports required by subpart I of this part;

(3) Include the results of monitoring used to assess the following quantities for external dose received during the year:

(i) The effective dose from external sources of radiation (equivalent dose to the whole body may be used as effective dose for external exposure);

(ii) The equivalent dose to the lens of the eye;

(iii) The equivalent dose to the skin; and 
(iv) The equivalent dose to the extremities.

(4) Include the following information for internal dose resulting from intakes received during the year:

(i) Committed effective dose;

(ii) Committed equivalent dose to any organ or tissue of concern; and

(iii) Identity of radionuclides.

(5) Include the following quantities for the summation of the external and internal dose:

(i) Total effective dose in a year;

(ii) For any organ or tissue assigned an internal dose during the year, the sum of the equivalent dose to the whole body from external exposures and the committed equivalent dose to that organ or tissue; and

(iii) Cumulative total effective dose.

(6) Include the equivalent dose to the embryo/fetus of a declared pregnant worker.

\subsubsection{How REX Fields are Used to Calculate 10 CFR 835 Protection Quantities}

The equations in Table 4.2 describe how REX sums dose data fields to obtain 10 CFR 835 protection quantities. The individual components of each data field are described in Table 4.1. The inclusion of $0.01 *$ [shallow - deep] in the summation for effective dose is a change implemented in response to the 2007 amendment to 10 CFR 835. It is a result of the introduction of a 0.01 tissue weighting factor for skin whereas previously there was none. In principle, this means that an individual who was exposed only to non-penetrating radiation will now have a small but positive whole body dose (effective dose) reported whereas under the old system they would not. The inclusion of $0.01 *$ [shallow - deep] in the summation for effective dose is allowed, but not required by 10 CFR 835 . It was adopted at Hanford because it is a more accurate representation of risk and avoids the perception of unaccounted dose.

\subsubsection{Mapping of Operational Quantities to 10 CFR 835 Protection Quantities}

The equations in Table 4.3 show how individual dosimeter results and dose assessment results from Table 4.1 contribute to the calculated 10 CFR 835 protection quantities. As can be seen, there is some conservatism in the calculated extremity dose for individuals wearing extremity dosimeters. This results in part from Hanford practice of allowing routinely issued rings to be worn independently from and for different wear periods than the chest dosimeter. Currently, the $\mathrm{H}_{\mathrm{p}}(0.07)$ result from the chest dosimeter is assigned to skin and extremity. Calculating the extremity dose from chest dosimeter results only for periods when rings were not worn is difficult when those periods are unknown. To eliminate conservatism, chest dosimeter and ring dosimeters would need to always be worn together. Allowing workers to wear a monthly issued ring only when needed, in conjunction with a quarterly or annually issued chest dosimeter reduces dosimetry costs and in most cases the conservatism in recorded extremity dose is offset by the reduction of cost. Another conservatism results from the fact that the highest extremity dosimeter result for any given monitoring period is the value used by REX in summation to obtain the reported equivalent dose to the extremities (EXTREMITY) on report cards to individuals. 
Table 4.2 Summation of Fields in REX to Obtain Protection Quantities

\begin{tabular}{|c|c|c|c|}
\hline $\begin{array}{l}10 \text { CFR } 835 \text { Protection } \\
\text { Quantity }\end{array}$ & $\begin{array}{l}10 \text { CFR } 835 \\
\text { Requirement }\end{array}$ & $\begin{array}{l}\text { Report Card } \\
\text { Nomenclature }\end{array}$ & $\begin{array}{l}\text { REX Components of } \\
\text { Quantity }\end{array}$ \\
\hline $\begin{array}{l}\text { Effective dose from external } \\
\text { sources, } \boldsymbol{E}\end{array}$ & 835.702(c)(3)(i) & ED & $\begin{array}{l}\text { deep + neutron + } \\
{\left[0.01^{*}(\text { shallow }- \text { deep })\right]}\end{array}$ \\
\hline $\begin{array}{l}\text { Equivalent dose to the whole body } \\
\text { from external sources, } \boldsymbol{H}_{\boldsymbol{w}}\end{array}$ & $\begin{array}{l}\text { 835.202(a)(2) } \\
835.702(\mathrm{c})(5)(\mathrm{ii})\end{array}$ & & $\begin{array}{l}\text { Same as for effective dose from } \\
\text { external sources }\end{array}$ \\
\hline $\begin{array}{l}\text { Equivalent dose to lens of eye from } \\
\text { external sources, } \boldsymbol{H}_{\text {eye }}\end{array}$ & 835.702(c)(3)(ii) & & eye + neutron \\
\hline Equivalent dose to lens of eye, $\boldsymbol{H}_{\text {eye }}$ & 835.202(a)(3) & LENS OF EYE & eye + neutron \\
\hline $\begin{array}{l}\text { Equivalent dose to skin from } \\
\text { external sources, } \boldsymbol{H}_{\text {skin }}\end{array}$ & $\begin{array}{l}\text { 835.202(a)(4) } \\
\text { 835.702(c)(3)(iii) }\end{array}$ & & shallow + neutron \\
\hline $\begin{array}{l}\text { Sum of equivalent dose to skin } \\
\text { from external sources and } \\
\text { committed equivalent dose to the } \\
\text { skin, } \boldsymbol{H}_{\text {skin }}+\boldsymbol{H}_{\text {skin,50 }}\end{array}$ & 835.202(a)(4) & SKIN & $\begin{array}{l}\text { shallow + neutron + } \\
\text { CEqD } \\
\text { skin }\end{array}$ \\
\hline $\begin{array}{l}\text { Equivalent dose to extremities from } \\
\text { external sources, } \boldsymbol{H}_{\text {extremity }}\end{array}$ & $\begin{array}{l}\text { 835.202(a)(4) } \\
\text { 835.702(c)(3)(iv) }\end{array}$ & & shallow + neutron + ring \\
\hline $\begin{array}{l}\text { Sum of equivalent dose to any } \\
\text { extremity from external sources } \\
\text { and committed equivalent dose to } \\
\text { the extremities, } \boldsymbol{H}_{\text {extremity }}+ \\
\boldsymbol{H}_{\text {extremity, } 50}\end{array}$ & $835.202(a)(4)$ & EXTREMITY & $\begin{array}{l}\text { shallow + neutron + ring + } \\
\text { CEqD } D_{\text {extremity }}\end{array}$ \\
\hline Committed effective dose, $\boldsymbol{E}_{50}$ & 835.702(c)(4)(i) & CED & CED (from Intertrac) \\
\hline $\begin{array}{l}\text { Committed equivalent dose to any } \\
\text { organ or tissue "of concern" } \boldsymbol{H}_{\mathrm{T}, 50}\end{array}$ & 835.702(c)(4)(ii) & CEqD & $\begin{array}{l}C E q D_{T} \text { for tissue } \mathrm{T} \text { (from } \\
\text { Intertrac) }\end{array}$ \\
\hline $\begin{array}{l}\text { Sum of the equivalent dose to the } \\
\text { whole body from external sources } \\
\text { and the committed equivalent dose } \\
\text { to any organ or tissue other than } \\
\text { skin or lens of eye, } \boldsymbol{E}+\boldsymbol{H}_{T, 50}\end{array}$ & $\begin{array}{l}\text { 835.202(a)(2) } \\
835.702(5)(\mathrm{ii})\end{array}$ & ORGAN DOSE & $\begin{array}{l}\text { deep }+ \text { neutron }+ \\
{\left[0.01^{\star}(\text { shallow }- \text { deep })\right]+} \\
C E q D_{T}\end{array}$ \\
\hline Total effective dose, $\boldsymbol{E}+\boldsymbol{E}_{50}$ & $\begin{array}{l}835.202(\mathrm{a})(1) \\
835.207 \\
835.208 \\
835.702(\mathrm{c})(5)(\mathrm{i})\end{array}$ & TED & $\begin{array}{l}\text { deep }+ \text { neutron }+ \\
{\left[0.01^{*}(\text { shallow }- \text { deep })\right]+} \\
C E D(\text { from Intertrac })\end{array}$ \\
\hline $\begin{array}{l}\text { Equivalent dose to the } \\
\text { Embryo/Fetus }\end{array}$ & $\begin{array}{l}\text { 835.206(a) } \\
835.702(6)\end{array}$ & $\begin{array}{l}\text { EMBRYO } \\
\text { /FETUS }\end{array}$ & $\begin{array}{l}\text { Deep + Neutron + } \\
\text { CEqD } \\
\text { embryo/fetus }\end{array}$ \\
\hline
\end{tabular}

(a) In the case where $\mathrm{T}=$ embryo/fetus, the actual quantity to be entered is currently under review, awaiting clarification from DOE. 
Table 4.3 Mapping of Dosimeter Results and Dose Assessment Results to 10 CFR 835 Protection Quantities

\begin{tabular}{|c|c|c|}
\hline $\begin{array}{l}10 \text { CFR } 835 \\
\text { Protection Quantity }\end{array}$ & $\begin{array}{c}10 \text { CFR } 835 \\
\text { Requirement }\end{array}$ & $\begin{array}{l}\text { Components of Quantity } \\
\text { (from Table 4.1) }\end{array}$ \\
\hline $\begin{array}{l}\text { Effective dose from external sources, } \\
\boldsymbol{E}\end{array}$ & 835.702(c)(3)(i) & $\begin{array}{l}H_{\mathrm{p}}(10)_{\gamma, w b}+E_{\gamma}+H_{\mathrm{p}}(10)_{\eta, w b}+E_{\eta}+0.01^{*} \\
{\left[H_{\mathrm{p}}(0.07)_{\beta \gamma, w b}+H_{\text {skin }}+H_{\text {non-U-skin }}-\right.} \\
\left.H_{\mathrm{p}}(10)_{\gamma, w b}-E_{\gamma}\right]\end{array}$ \\
\hline $\begin{array}{l}\text { Equivalent dose to the whole body } \\
\text { from external sources, } \boldsymbol{H}_{w b}\end{array}$ & $\begin{array}{l}\text { 835.202(a)(2) } \\
835.702(\mathrm{c})(5)(\mathrm{ii})\end{array}$ & $\begin{array}{l}\text { Same as for Effective dose from external } \\
\text { sources }\end{array}$ \\
\hline $\begin{array}{l}\text { Equivalent dose to lens of eye from } \\
\text { external sources, } \boldsymbol{H}_{\text {eye }}\end{array}$ & 835.702(c)(3)(ii) & $H_{\mathrm{p}}(3)_{\beta \gamma, w b}+H_{\text {eye }}+H_{\mathrm{p}}(10)_{\eta, w b}+E_{\eta}$ \\
\hline Equivalent dose to lens of eye, $\boldsymbol{H}_{\text {eye }}$ & 835.202(a)(3) & $H_{\mathrm{p}}(3)_{\beta \gamma, w b}+H_{e y e}+H_{\mathrm{p}}(10)_{\eta, w b}+E_{\eta}$ \\
\hline $\begin{array}{l}\text { Equivalent dose to skin from external } \\
\text { sources, } \boldsymbol{H}_{\text {skin }}\end{array}$ & $\begin{array}{l}\text { 835.202(a)(4) } \\
\text { 835.702(c)(3)(iii) }\end{array}$ & $\begin{array}{l}H_{\mathrm{p}}(0.07)_{\beta \gamma, w b}+H_{s k i n}+H_{\text {non-U-skin }}+ \\
H_{\mathrm{p}}(10)_{\eta, w b}+E_{\eta}\end{array}$ \\
\hline $\begin{array}{l}\text { Sum of equivalent dose to skin from } \\
\text { external sources and committed } \\
\text { equivalent dose to the skin, } \boldsymbol{H}_{\text {skin }}+ \\
\boldsymbol{H}_{\text {skin, } 50}\end{array}$ & 835.202(a)(4) & $\begin{array}{l}H_{\mathrm{p}}(0.07)_{\beta \gamma, w b}+H_{\text {skin }}+H_{\text {non-U-skin }}+ \\
H_{\mathrm{p}}(10)_{\eta, w b}+E_{\eta}+H_{\text {skin, } 50}\end{array}$ \\
\hline $\begin{array}{l}\text { Equivalent dose to extremities from } \\
\text { external sources, } \boldsymbol{H}_{\text {extremity }}\end{array}$ & $\begin{array}{l}\text { 835.202(a)(4) } \\
\text { 835.702(c)(3)(iv) }\end{array}$ & $\begin{array}{l}H_{\mathrm{p}}(0.07)_{\beta \gamma, w b}+H_{\text {skin }}+H_{\text {non-U-skin }}+ \\
H_{\mathrm{p}}(10)_{\eta, w b}+E_{\eta}+H_{\mathrm{p}}(0.07)_{\beta \gamma, e x t r}+H_{\text {extr }}+ \\
H_{\text {non-U-extr }}\end{array}$ \\
\hline $\begin{array}{l}\text { Sum of equivalent dose to any } \\
\text { extremity from external sources and } \\
\text { committed equivalent dose to the } \\
\text { extremities, } \boldsymbol{H}_{\text {extremity }}+\boldsymbol{H}_{\text {extremity }, 50}\end{array}$ & 835.202(a)(4) & $\begin{array}{l}H_{\mathrm{p}}(0.07)_{\beta \gamma, w b}+H_{s k i n}+H_{\text {non-U-skin }}+ \\
H_{\mathrm{p}}(10)_{\eta, w b}+E_{\eta}+H_{\mathrm{p}}(0.07)_{\beta \gamma, \text { extr }}+H_{\text {extr }}+ \\
H_{\text {non-U-extr }}+H_{\text {extremity,50 }}\end{array}$ \\
\hline Committed effective dose, $\boldsymbol{E}_{50}$ & 835.702(c)(4)(i) & $E_{50}$ \\
\hline $\begin{array}{l}\text { Committed equivalent dose to any } \\
\text { organ or tissue "of concern" } \boldsymbol{H}_{\mathrm{T}, 50}\end{array}$ & 835.702(c)(4)(ii) & $H_{T, 50}$ \\
\hline $\begin{array}{l}\text { Sum of the equivalent dose to the } \\
\text { whole body from external sources and } \\
\text { the committed equivalent dose to any } \\
\text { organ or tissue other than skin or lens } \\
\text { of eye, } \boldsymbol{H}_{w b}+\boldsymbol{H}_{T, 50}\end{array}$ & $\begin{array}{l}\text { 835.202(a)(2) } \\
835.702(5)(\mathrm{ii})\end{array}$ & $\begin{array}{l}H_{\mathrm{p}}(10)_{\gamma, w b}+E_{\gamma}+H_{\mathrm{p}}(10)_{\eta, w b}+E_{\eta}+0.01^{*} \\
{\left[H_{\mathrm{p}}(0.07)_{\beta \gamma, w b}+H_{\text {skin }}+H_{\text {non-U-skin }}-\right.} \\
\left.H_{\mathrm{p}}(10)_{\gamma, w b}-E_{\gamma}\right]+H_{T, 50 \text { (T } \neq \text { skin or lens of eye })}\end{array}$ \\
\hline Total effective dose, $\boldsymbol{E}+\boldsymbol{E}_{50}$ & $\begin{array}{l}835.202(a)(1) \\
835.207 \\
835.208 \\
835.702(c)(5)(i)\end{array}$ & $\begin{array}{l}H_{\mathrm{p}}(10)_{\gamma, w b}+E_{\gamma}+H_{\mathrm{p}}(10)_{\eta, w b}+E_{\eta}+0.01^{*} \\
{\left[H_{\mathrm{p}}(0.07)_{\beta \gamma, w b}+H_{\text {skin }}+H_{\text {non-U-skin }}-\right.} \\
\left.H_{\mathrm{p}}(10)_{\gamma, w b}-E_{\gamma}\right]+E_{50}\end{array}$ \\
\hline $\begin{array}{l}\text { Equivalent dose to the } \\
\text { Embryo/Fetus }\end{array}$ & $\begin{array}{l}\text { 835.206(a) } \\
835.702(6)\end{array}$ & $H_{\mathrm{p}}(10)_{\gamma, w b}+H_{\mathrm{p}}(10)_{\eta, w b}+H_{e m b / f e t u s, \text { in utero }}$ \\
\hline
\end{tabular}

(a) 835.206(a) expresses the limit as equivalent dose. 835.702(c)(6) requires that equivalent dose be recorded. Neither of these paragraphs makes a distinction between equivalent dose from external and internal sources. Presumably internal dose would not be a 50 year commitment but rather the equivalent dose from conception to birth. DOE G 441.1-1C clearly states that for internal doses, the quantity to be assessed is equivalent dose from conception to birth. However, RCTP 2009-01 specifies that the equivalent dose to be recorded for the embryo/fetus is to include committed equivalent dose. [In no place does DOE use the term committed to mean anything other than a 50 year commitment.] Historically, the approach has been to calculate dose for the gestation period. The Hanford IDP has proposed continuing this practice, but to assess effective dose (in utero) using coefficients from ICRP 88. The actual quantity that will be entered into the REX field $\mathrm{CE}_{\mathrm{q}} \mathrm{D}_{\mathrm{T}}$ where $\mathrm{T}=$ embryo/fetus, and the method of calculation, is yet to be determined. The issue is currently under review, pending clarification from DOE. 


\subsubsection{Inconsistencies with DOE Definition and Use of Protection Quantities}

In the body of the 2007 amendment to 10 CFR 835, DOE's use of the term "equivalent dose" was inconsistent with the ICRP/ICRU definitions of this term and DOE's own definition of this term. (Use of the term "dose equivalent" was avoided all together.) The result was ambiguous meaning at several places in the text of the rulemaking leading to questions of interpretation. DOE's "Discussion of Changes to 10 CFR Part 835" (section IV of preamble to rulemaking), and subsequent DOE guidance given in DOE G 441.1-1C Radiation Protection Programs Guide (DOE 2008) and RCTP 2009-01 Technical Position Clarifying Dose Assessment and Recording (DOE 2009) has addressed many of the ambiguities but a few remain. The discussion below identifies some of the inconsistencies and documents how they have been resolved by HEDP.

10 CFR 835.2(b) defines equivalent dose as follows:

"Equivalent dose $\left(\mathrm{H}_{\mathrm{T}}\right)$ means the product of average absorbed dose $\left(\mathrm{D}_{\mathrm{T}, \mathrm{R}}\right)$ in rad (or gray) in a tissue or organ ( $\mathrm{T}$ ) and a radiation (R) weighting factor $\left(w_{R}\right)$. For external dose, the equivalent dose to the whole body is assessed at a depth of $1 \mathrm{~cm}$ in tissue; the equivalent dose to the lens of the eye is assessed at a depth of $0.3 \mathrm{~cm}$ in tissue, and the equivalent dose to the extremity and skin is assessed at a depth of $0.007 \mathrm{~cm}$ in tissue. Equivalent dose is expressed in units of rems (or Sv)."

Problem:

DOE has defined a protection quantity based on ICRP 60 but used it like an operational quantity. In principle, the equivalent dose to the whole body, lens of eye, skin or extremity can be calculated based on the ICRP definition of equivalent dose but not using the DOE definition above. The absorbed dose used to calculate the equivalent dose must be averaged over the mass of a specified tissue (i.e. volume). The specification of a depth by DOE for these "tissues" permits calculation of dose at a single point or dose averaged over a set of points representing a surface, but not a dose averaged over a volume of tissue.

\section{Resolution:}

DOE's specification of a depth for assessment of equivalent dose to these tissues implies DOE's intended use of operational quantities as estimates for these protection quantities. DOE G 441.1-1C and RCTP 2009-01 recommend the use of operational quantities as estimates of equivalent dose to these "tissues".

Hanford practice will therefore be as follows: For exposures in uniform external radiation fields, $\mathrm{H}_{\mathrm{p}}(10)$ will be used as an estimate for "equivalent dose to the whole body", $\mathrm{H}_{\mathrm{p}}(3)$ will be used as an estimate for "equivalent dose to the lens of the eye" and $\mathrm{H}_{\mathrm{p}}(0.07)$ will be used as an estimate for "equivalent dose to the skin" and "equivalent dose to the extremity" (for extremity dosimeters). For exposures in non-uniform external radiation fields, a value of effective dose, E, assessed from multiple dosimeter data will be used for "equivalent dose to the whole body". Values of $\mathrm{H}_{\text {eye }}, \mathrm{H}_{\text {skin }}$ and $\mathrm{H}_{\text {extremity }}$ assessed from multiple dosimeter data will be used for "equivalent dose to the lens of the eye", "equivalent dose to the skin" and "equivalent dose to the extremity" respectively. For non-uniform 
exposures of the skin or extremity from contamination on skin or clothing, values of $\mathrm{H}_{\text {skin }}$ and $\mathrm{H}_{\text {extremity }}$ will be assessed using VARSKIN 3 or other calculational methods.

Notes:

It is reasonable to assume that in uniform fields, the calculated values for $\mathrm{E}$ and for "equivalent dose to the whole body" would be the same. However, it is not clear that in non-uniform fields, calculated values for $\mathrm{E}$ and for "equivalent dose to the whole body" (based on strict definitions of effective dose and equivalent dose) would be the same. Nevertheless, RCTP 2009-01 recommends using effective dose for "equivalent dose to the whole body" and this is the approach adopted by HEDP. 


\subsection{Dosimetry System Design, Calibration, and Dose Response}

To the extent possible, the technical bases of HEDP dosimetry practices are determined from laboratory studies, field measurements and data available in the scientific literature. The 318 Building calibrations facility is a National Institute of Standards and Technology (NIST) accredited calibration laboratory for ionizing radiation with the capability to provide dosimeter irradiations under a variety of conditions. Essentially all source irradiations are traceable to NIST. Performance of HEDP dosimetry systems is tested under a range of radiation and environmental conditions expected in Hanford work environments. In addition, HEDP is required to meet DOELAP requirements as described in, Standard for the Performance Testing of Personnel Dosimetry Systems DOE/EH-0027 (DOE 1986a); and Handbook for the Department of Energy Accreditation Program for Personnel Dosimetry System DOE/EH-0026 (DOE 1986b).

To meet Hanford external dosimetry objectives, the HEDP provides centralized dosimetry services and technical support to all Hanford contractors. A TLDbased system was selected as the primary dosimetry method because of the TLDs demonstrated performance during approximately three decades of use and several advantages over other types of dosimeter technology.

The HEDP provides several types of dosimeters, processing service, and technical support. These can be combined into five general areas as follows:

- $\quad$ whole body dosimetry

- $\quad$ extremity dosimetry

- $\quad$ area dosimetry

- environmental dosimetry

- nuclear accident dosimetry

Each of these areas employs thermoluminescent dosimetry capabilities. Other HEDP dosimetry techniques include neutron activation analysis to evaluate biological samples and components of Hanford nuclear accident dosimeters. The TLD systems may be considered to involve five functional components:

- $\quad$ TLD phosphors

- $\quad$ TLD cards or Chipstrates ${ }^{\circledR}$

- TLD holders

- $\quad$ TLD readers

- data storage, data analysis, data reporting and record systems. 


\subsection{Characteristics of TLD Phosphors Used in HEDP Dosimeters}

The successful use of thermoluminescence as a means of measuring radiation dose has been demonstrated for many years (ICRU 1992). Hanford has used TLDs for personnel dosimetry since 1971 (Wilson 1987), for environmental dosimetry since 1972 (Denham et al. 1972), and for nuclear accident dosimetry since 1977 (Glenn and Bramson 1977).

\subsubsection{Lithium Fluoride}

Lithium fluoride ( $\mathrm{LiF}$ ), with its low atomic number and simple cubic lattice, was one of the first phosphors to become commercially available for personnel dosimetry applications. This phosphor has many good performance characteristics including near-tissue-equivalent response, unaffected (relatively) by environmental conditions (i.e., humidity, normal working temperatures, etc.), and linear dose response at occupational dose levels. The phosphor also has some undesirable performance characteristics such as supralinearity at higher dose levels, complicated annealing behavior, response to light, and relatively poor sensitivity (Horowitz 1984). These issues require sophisticated evaluation of the dosimeter processing data to determine personnel dose.

The original LiF was made by the Harshaw Chemical Company before 1954. However, systematic studies of various activators and activator combinations led to the material that is now widely used. Various types of LiF phosphors are available, covering a wide variety of lithium enrichments. These include TLD600 (approximately $95.6 \%{ }^{6} \mathrm{Li}$ and $4.4 \%{ }^{7} \mathrm{Li}$ ) and TLD-700 (approximately 99.99\% ${ }^{7} \mathrm{Li}$ and approximately $0.01 \%{ }^{6} \mathrm{Li}$ ). The natural isotopic abundance of lithium fluoride is $7.5 \%{ }^{6} \mathrm{Li}$ and $92.5 \%{ }^{7} \mathrm{Li}$. Both TLD-600 and TLD-700 contain trace elements shown in Table 5.1 (Becker et al. 1970).

Table 5.1. Trace Elements in Lithium Fluoride Thermoluminescent Dosimeters

\begin{tabular}{||l|c|}
\hline \multicolumn{1}{|c|}{ Contaminant } & Approximate Contents, ppm \\
\hline Aluminum & 20 \\
\hline Calcium & 6 \\
\hline Magnesium & 300 \\
\hline Silicon & 40 \\
\hline Titanium & 5 \\
\hline
\end{tabular}

In general, magnesium and titanium are believed to be the trace elements of primary dosimetric importance in LiF TLD (Robertson and Gilboy 1971), and for this reason the phosphor is typically noted as $\mathrm{LiF}: \mathrm{Mg}, \mathrm{Ti}$. 
Dosimetry technology has evolved using LiF phosphors for beta, photon, and neutron radiation dose measurement. $\mathrm{LiF}$ has an additional advantage. The isotope ${ }^{6} \mathrm{Li}$ has a relatively large capture cross-section (approximately 953 barns) for thermal neutrons, and because this isotope is present in natural lithium (i.e., approximately $7 \%$ ), LiF makes an excellent detector of thermal neutrons. In contrast, ${ }^{7} \mathrm{Li}$ has an extremely small capture cross-section (approximately 0.037 barns). Natural lithium can be made more sensitive by enriching it in the isotope ${ }^{6} \mathrm{Li}$. Likewise, it can be made almost insensitive to thermal neutrons by depleting the lithium of ${ }^{6} \mathrm{Li}$.

When a radiation worker is irradiated with fast neutrons, there is little probability that the ${ }^{6} \mathrm{Li}$ in the personnel dosimeter will capture an incident neutron. It is more likely that some fraction of the fast neutrons will be moderated (slowed) by the worker's body, be reflected backwards, and be captured by the ${ }^{6} \mathrm{Li}$ in the TLD. This "albedo effect" is the basis for neutron dosimetry in the HEDP TLD system.

The following Hanford dosimeters use the LiF phosphor:

- $\quad$ Hanford Standard Dosimeter (HSD)

- $\quad$ Hanford Combination Neutron Dosimeter (HCND)

- $\quad$ EXT-RAD Ring Dosimeter

- Hanford Environmental Dosimeter

- $\quad$ Hanford Nuclear Accident Dosimeters.

All of the LiF phosphors contained in these dosimeters are 3.2-mm (1/8-in.) squares in the form of hot pressed chips. Three different thicknesses of LiF chips are used: $0.15 \mathrm{~mm}$ (0.006 in.), $0.38 \mathrm{~mm}$ (0.015 in.), and $0.89 \mathrm{~mm}$ (0.035 in.). The phosphors used in each dosimeter type are given in Sections 5.3 through 5.10.

\subsubsection{Calcium Fluoride}

Calcium fluoride, $\mathrm{CaF}_{2}$ :Dy, (known commercially as TLD-200) is used in the Hanford environmental dosimeter. The TLD 200 phosphors used by HEDP are 3.2-mm (1/8-in.) squares in the form of hot pressed chips. Only one thickness of chip is used: $0.89 \mathrm{~mm}$ (0.035 in.). Unacceptable fading, as much as $10 \%$ per month, can occur without a post irradiation annealing. HEDP TLD readers are programmed to apply a pre-heat anneal as part of the readout process to minimize fading.

\subsubsection{Physical Form}

The hot pressed form of the TLD phosphors is produced by the vendor through compression of blended polycrystalline material into a slug at an elevated temperature. Blending of source material from different crystal growths with different glow curve structure and sensitivity results in uniform glow curve structure and sensitivity within the chips produced. The fused polycrystalline slug is sliced and diced to produce individual chips, which are then ground to uniform thickness. While loose chips may be annealed at high temperature and are easily 
handled and washed, careful handling is necessary to avoid mechanical effects (e.g., triboluminescence). Because most HEDP dosimeters use chips mounted on a substrate (card or chipstrate), physical handling of the individual chips is currently necessary only in nuclear accident dosimeter fabrication, disassembly, and processing.

\subsubsection{Linearity of Dose Response}

The dose-response curve represents the average TL output as a function of dose. The dose-response curves for these TLD phosphors are linear in the dose range for routine results, followed in the case of LiF by a supra-linear range for doses greater than 100 rad. Maximum over response occurs at about 50,000 rad, above which TL yield decreases. Within an absorbed dose range of $10 \mathrm{mrad}$ to $100 \mathrm{rad}$, there is an average deviation of $4.5 \%$ from linearity for all TLD phosphors. The linear dose-response curves from $10 \mathrm{mrad}$ to $10 \mathrm{rad}$ have linear regression coefficients of 0.9993 or greater for all phosphors tested (Harshaw 1988).

\subsubsection{Sensitivity}

Sensitivity, defined as the TL output per unit mass and unit absorbed dose, is influenced by many factors (e.g., type of phosphor, the type and features of the reader, heat treatment, etc.). Typically, only the relative sensitivity is quantified. As used within the HEDP, (i.e. HEDP annealing and readout protocols), the sensitivity of $\mathrm{CaF}_{2}$ :Dy relative to $\mathrm{LiF}: \mathrm{Mg}$, Ti when irradiated with gamma radiation from ${ }^{60} \mathrm{Co}$, is approximately a factor of 18 on a per unit mass basis (Rathbone, Endres, and Antonio, 1994).

In general, there is a decrease in sensitivity for TLD phosphors after many reuses. For all TLD phosphors contained in HEDP dosimeters, a loss of sensitivity of less than $2 \%$ is expected during as many as 500 re-uses. In addition, all phosphors exhibit less than $0.8 \%$ degradation for every 100 re-uses, up to a total of 2000 reads (Harshaw 1988).

\subsubsection{Fading}

Fading is defined as a loss of TL signal with time since exposure. Fading may be due to thermally or optically stimulated release of trapped electrons, or a combination of both. Marked thermal fading is observed when the glow curve contains one or more low-temperature peaks. The $\mathrm{LiF}$ phosphors without $80^{\circ} \mathrm{C}$ oven annealing exhibit $<10 \%$ loss of signal per month at $25^{\circ} \mathrm{C}$ (Harshaw 1988), after an initial 24-hour fading period, following exposure to gamma rays from ${ }^{137} \mathrm{Cs}$. Use of a pre-irradiation oven anneal for 16 hours at $80^{\circ} \mathrm{C}$ reduces fade in $\mathrm{LiF}$ phosphors used by HEDP to less than $15 \%$ per year. ${ }^{\text {(a) }} \mathrm{CaF}_{2}$ :Dy exhibits a much larger fading rate.

(a) W. V. Baumgartner, "Study of Environmental Buildup and Fade for 8825 Card,” October 11, 1994, letter to HEDP file. 


\subsubsection{Annealing}

Various types of thermal annealing are conducted to minimize the effect of fading. For loose LiF chips, a high-temperature oven anneal for 1.5 hours at $400^{\circ} \mathrm{C}$ followed by a quench to room temperature, then a low temperature oven anneal at $80^{\circ} \mathrm{C}$ for 16 hours is used to minimize fading. For chips mounted in cards, where the temperature cannot exceed $312^{\circ} \mathrm{C}$ without destroying the Teflon $^{\circledR}$, the chips are reader annealed at $300^{\circ} \mathrm{C}$ for $3-10$ seconds depending on phosphor and application, then oven annealed at $80^{\circ} \mathrm{C}$ for 16 hours. The 16 hour $80^{\circ} \mathrm{C}$ oven anneal reduces the number of low temperature traps in the $\mathrm{LiF}$ material. A pre-read anneal in the TLD reader is used with $\mathrm{CaF}_{2}$ :Dy to eliminate the lower-temperature peaks, which greatly reduces the fading rate. Whenever possible, HEDP uses annealing techniques that reduce fading and the associated uncertainty in dose results for personnel and environmental dosimeters.

\subsubsection{Photon Energy Dependence}

The photon energy response of a TL phosphor depends primarily on its effective atomic number. The TL response of a phosphor is usually normalized to a particular photon energy. The theoretical energy response of LiF shows an over response (relative to ${ }^{137} \mathrm{Cs}$ ) in the 20 to $100 \mathrm{keV}$ range with a maximum of about $40 \%$ between 20 and $30 \mathrm{keV}$. This may be due to one or more factors, including absorption of the soft x-rays in the material or grain size effects (Horowitz 1984). For most applications, LiF elements are considered to be approximately tissue equivalent, with little energy dependence for photon energies greater than $100 \mathrm{keV}$. For $\mathrm{CaF}_{2}$, a much higher photon energy dependence is observed because of the relatively high atomic number (16.3), compared with tissue (7.4) (McKinlay 1981). Energy dependence studies of TL phosphors have been published by several authors (ICRU 1992).

\subsubsection{Batch Uniformity}

The basic design of the HEDP dosimeters, which includes collection and storage of element-specific correction coefficients, does not require strict uniformity in response for all TLD elements. However, upon testing by the vendor, the raw response of 3600 randomly selected elements from the vendor inventory varied by less than 30\% (Harshaw 1988). Typical standard deviations for raw response in 15 mil LiF chips being calibrated and acceptance tested for individual sensitivity are about $10 \%$.

\subsubsection{Reproducibility}

Eighty TLD elements were randomly selected from production inventory and repeatedly exposed and read. Exposures were $100 \mathrm{mR}$ from a ${ }^{137} \mathrm{Cs}$ source. The coefficient of variation (C.V.) in readings was calculated for each chip. The mean C.V. for the sample of 80 elements was $1.3 \%$ (Harshaw 1988). To avoid the need for frequent re-calibration of cards and chipstrates, it is critical that each dosimeter element maintain its initial readout characteristics for a reasonable life span of exposure/readout cycles. The average change in sensitivity over 500 re-uses is less than $2 \%$ as determined from vendor testing (Harshaw 1988). 


\subsubsection{Residual TL}

The residual TL was determined by the vendor for 80 randomly selected TLD elements, after giving them a dose of 500 mrad from ${ }^{137} \mathrm{Cs}$ gamma radiation. The ratio of the re-read responses to the read responses for all elements was less than 0.5\% (Harshaw 1988).

\subsection{Characteristics of HEDP Dosimeter Cards}

The TLD chips contained in HEDP dosimeters are mounted in aluminum dosimeter cards and encapsulated between two sheets of Teflon ${ }^{\circledR}$ that are each 0.05-mm thick. Each card is marked with a seven-digit identification (ID) number in both human-readable and bar-coded format. The first digit of the ID number is used to identify the dosimeter type (i.e., standard, neutron, environmental, etc.). Because the dosimeter cards may contain one or more types of TLD phosphors (e.g., TLD-200, TLD-600, or TLD-700), this unique ID number is necessary to maintain card-specific read cycle parameters (i.e., calibration values, timetemperature profiles, etc.).

Processing of HEDP cards is expected to result in less than one in 10,000 erroneous dosimeter identifications, including both the dosimeter type and serial number (Harshaw 1988). Each card is designed to withstand a minimum of 500 readout cycles without decrease in its mechanical performance, including identification and reading of dosimeter type and serial number. Acceptance tests are conducted of all HEDP dosimeter cards and holders to ensure proper construction and performance under operational conditions. These tests are described in the following sections.

\subsubsection{Physical Testing}

Both dosimeter cards and holders are tested. For cards, the general appearance of the card is visually inspected, the integrity of the Teflon ${ }^{\circledR}$ is examined, and the first digit of the card ID number is compared to ensure that it corresponds to the type of dosimeter cards procured. For holders, each one is examined for physical damage, for proper clearances to allow insertion of the card in only one orientation, and for the quality of the "O-ring" gasket, which minimizes moisture and dust penetration. An eddy current meter is used to check the type and thickness of each of the metallic filters. Once these tests are completed, a visual test to detect light penetration of the Mylar ${ }^{\circledR}$ window on the Hanford standard holders is performed.

\subsubsection{Unique Permanent Identification Number}

Before any irradiation testing, each card is processed through the reader system to determine if the card barcode label can be read and the card processed. The file of processed cards is uploaded to the HEDP External Dosimetry computer system where checks are made to ensure that the card's permanent ID number is unique.

\subsubsection{Testing for Phosphor Type}

Following reader- and oven-annealing preparation steps, cards are exposed to a

${ }^{252} \mathrm{Cf}$ neutron source centered in a stainless steel-lined sphere (30-cm diameter) 
containing $\mathrm{D}_{2} \mathrm{O}$. This source provides an abundance of thermalized neutrons sufficient to distinguish among TLD-100, TLD-600, and TLD-700 phosphors. To distinguish between the neutron-insensitive TLD-200 and TLD-700 phosphors which have vastly different beta-gamma sensitivities, the sensitivity factors or element correction coefficients (ECC's) obtained during card calibration (Section 5.3.3) are evaluated. The combination of these tests ensures that the correct phosphors are contained in each position of HEDP cards and that these phosphors demonstrate acceptable performance.

\subsubsection{Light Testing}

LiF TLD phosphors and Teflon ${ }^{\circledR}$ substrates generally have a small degree of sensitivity to light. However, some individual chips and or Teflon ${ }^{\circledR}$ enclosures are significantly more sensitive than others, and demonstrate unacceptable sensitivity to light. For this reason, all HEDP cards are tested for light sensitivity. Following reader- and oven-annealing preparation steps, cards are exposed for two hours under routine laboratory lighting conditions (fluorescent light with ultraviolet filter) and then processed. The data are screened to detect any unacceptable results.

\subsubsection{Time-Temperature Profile (TTP)}

During automated processing with the Harshaw 8800 reader system, each TLD card type has its own specific processing protocol because of the differences in heating and annealing characteristics of the different phosphors. Typical reader processing setup parameters are listed in Table 5.2. These time temperature parameters, along with specific information pertaining to the regions of the glow curve to be used for dose calculation and quality control, are referred to as the time temperature profile (TTP).

Table 5.2. Typical Parameters for Reader Processing Setup

\begin{tabular}{||l|l||}
\hline \multicolumn{1}{|c|}{ Description } & \multicolumn{1}{c|}{ Setting } \\
\hline Preheat temperature & $50^{\circ} \mathrm{C}$ \\
\hline Preheat time & 0 seconds \\
\hline Temperature rate & $25^{\circ} \mathrm{C} /$ second \\
\hline Maximum temperature & $300^{\circ} \mathrm{C}$ \\
\hline Acquire time & $13-1 / 3$ seconds \\
\hline Annealing temperature & $300^{\circ} \mathrm{C}$ \\
\hline Annealing time & 10 seconds \\
\hline
\end{tabular}

In Table 5.2, the acquire time (data acquisition time) represents the time during which glow information is recorded and may be less than the actual heating time. Temperature increases at about $100^{\circ} \mathrm{C} / \mathrm{s}$ from ambient to the designated preheat temperature and is held at that temperature for the designated time. In the example given, it takes less than one second to reach the prescribed $50^{\circ} \mathrm{C}$ preheat temperature. During preheat, no data are acquired. Data acquisition begins, at the start of the $25^{\circ} \mathrm{C} / \mathrm{sec}$ temperature ramp and continues for the specified acquisition time. In the example given, it takes 10 seconds for the temperature to increase from $50^{\circ} \mathrm{C}$ to $300^{\circ} \mathrm{C}$ after which the temperature remains at $300^{\circ} \mathrm{C}$ for an additional 3.33 seconds. Thus the data acquisition time is 13.33 seconds. After 
data acquisition ends, the temperature increases to the prescribed anneal temperature at a rate of about $50^{\circ} \mathrm{C} / \mathrm{s}$ and remains there for the prescribed anneal time, then drops quickly to room temperature. In the example given, the temperature remains at $300^{\circ} \mathrm{C}$ for 10 seconds followed by a rapid drop to room temperature. An illustration showing possible variations for a TTP with Harshaw 8800 and 6600 TLD readers is shown in Figure $\mathbf{5 . 1}$

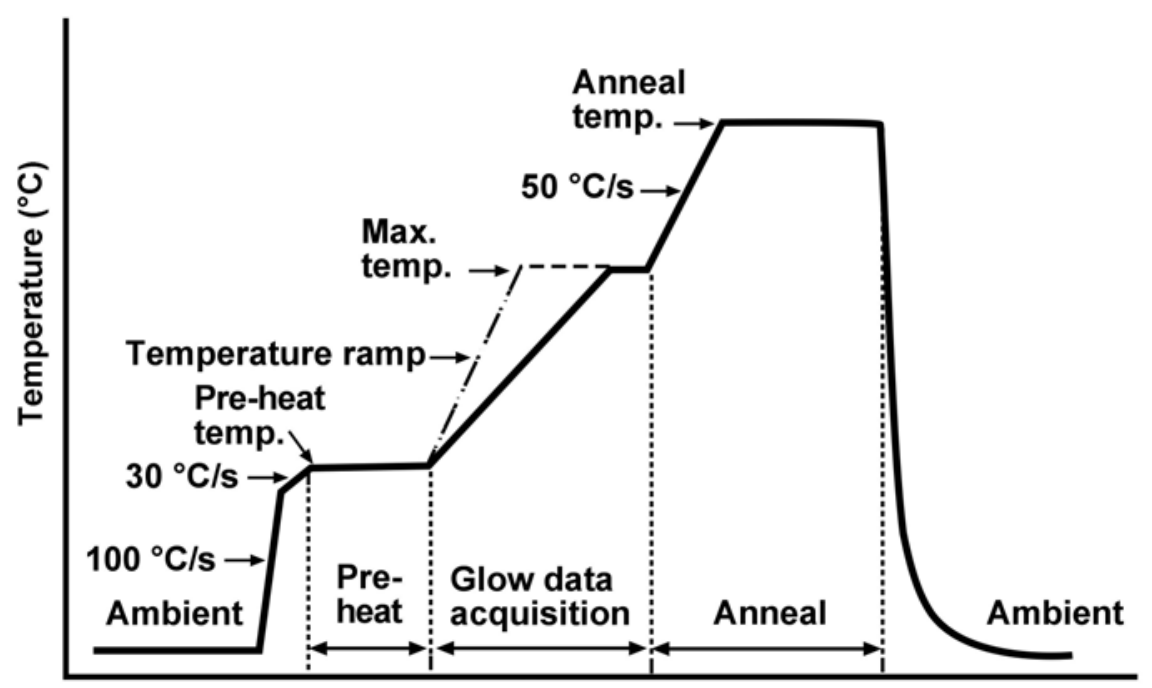

Time (s)

Figure 5.1. Variations on a Time Temperature Profile

Specification of correct TTP parameters and consistency of the temperature applied to all cards are very important to achieving quality performance and reproducible glow curves. The automated reader system maintains a data file showing the TTP used for processing each card. TTPs are specified for each type of card. In addition, the reader system maintains a history of changes made to the configuration of each TTP. This is described further under subsequent sections for each type of dosimeter card.

\subsubsection{Glow Curve}

Figure 5.2 shows a typical glow curve for a LiF:Mg,Ti chip from a chipstrate extremity dosimeter that has been exposed to $500 \mathrm{mR}$. The chip was given a reader anneal and a 16 hour $80^{\circ} \mathrm{C}$ oven anneal, followed by $500 \mathrm{mR}$ exposure to ${ }^{60}$ Co before being read using the TTP shown in Table 5.2. The abscissa represents time, where 200 channels are equal to the total acquisition time (13.33 seconds for this TTP). The ordinate represents glow intensity in arbitrary units scaled such that the glow curve maximum is plotted at full scale. The ordinate also represents temperature where $0{ }^{\circ} \mathrm{C}$ is at the origin and seven scale divisions represents a span of approximately $300^{\circ} \mathrm{C}$. 


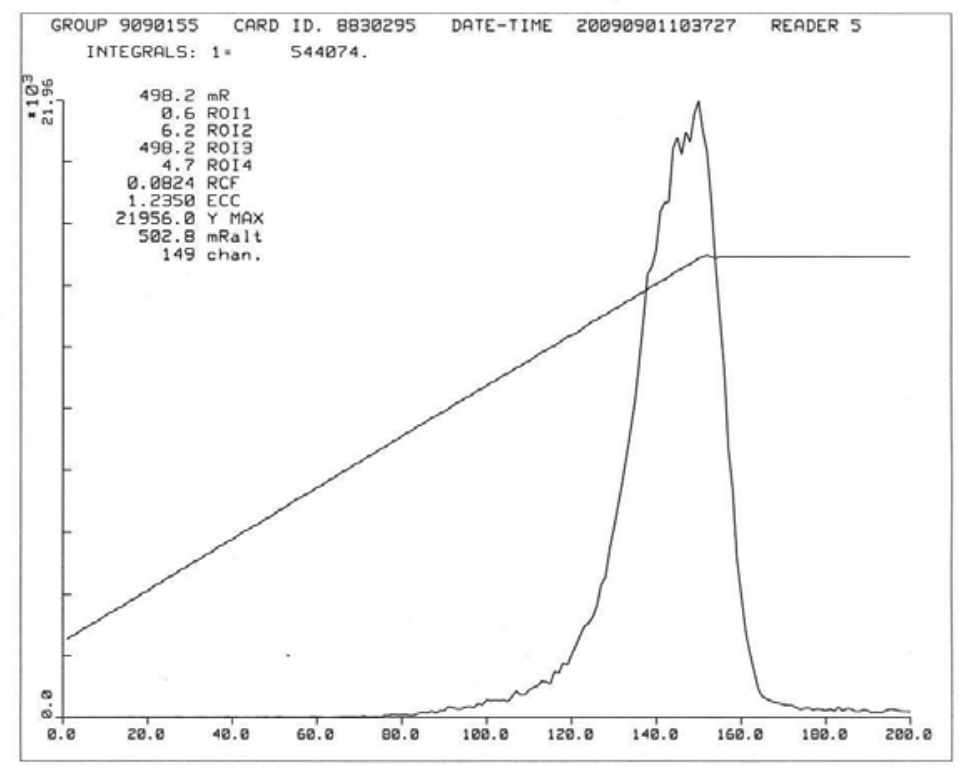

Figure 5.2 Typical LiF glow curve with standard TTP

\subsection{HEDP Dosimetry System}

A dosimetry system consists of dosimeters, dosimeter readers, and a methodology for calibration and dose calculation as embodied in the processing procedures and software. The design features and performance characteristics of the Harshaw TLD readers used in the HEDP dosimetry system are described in Chapter 3. The dosimeters and calibration / dose calculation methodology are described in this section.

An HEDP dosimeter consists of a TLD card, complete with the appropriate complement of TLD phosphors, and a holder, used to maintain the card in a protected and light-sealed environment. The card holder is sealed with a gasket to prevent liquids from entering. The card cavity is keyed such that an improperly inserted TLD card will prevent the two halves of the card holder from closing. The cards are removed from their holders during processing. Individual TLD chips are used in the HEDP extremity and nuclear accident dosimeters. Table 5.3 describes the phosphors used in each of the HEDP dosimeters. 
Table 5.3. Hanford Dosimeter Specifications

\begin{tabular}{|c|c|c|c|}
\hline $\begin{array}{l}\text { Dosimeter Type } \\
\text { (Card Type) } \\
\text { (Holder Type) }\end{array}$ & Card/Holder ID\# & Description of Active Elements ${ }^{(a, d)}$ & Description of Holder ${ }^{(b, c, d)}$ \\
\hline $\begin{array}{l}\text { HSD } \\
\text { (8825BPN card) } \\
\text { (8825 holder) }\end{array}$ & $\begin{array}{l}00 \mathrm{xxxxx} / \\
000 \mathrm{xxxxx}\end{array}$ & $\begin{array}{l}\text { Element 1: TLD-700 }\left(100 \mathrm{mg} / \mathrm{cm}^{2}\right) \\
3.175 \text { x } 3.175 \text { x } 0.381 \mathrm{~mm}^{3} \\
\text { Element 2: TLD-700 }\left(100 \mathrm{mg} / \mathrm{cm}^{2}\right) \\
3.175 \text { x } 3.175 \times 0.381 \mathrm{~mm}^{3} \\
\text { Element 3: TLD-700 }\left(40 \mathrm{mg} / \mathrm{cm}^{2}\right) \\
3.175 \text { x 3.175 x } 0.152 \mathrm{~mm}^{3} \\
\text { Element 4: TLD-600 }\left(100 \mathrm{mg} / \mathrm{cm}^{2}\right) \\
3.175 \times 3.175 \times 0.381 \mathrm{~mm}^{3}\end{array}$ & $\begin{array}{l}\text { Front: ABS } 242 \mathrm{mg} / \mathrm{cm}^{2}, \mathrm{Cu} 91 \mathrm{mg} / \mathrm{cm}^{2} \\
\text { Back: ABS } 173 \mathrm{mg} / \mathrm{cm}^{2} \\
\text { Front: PTFE+ABS } 1000 \mathrm{mg} / \mathrm{cm}^{2} \\
\text { Back: ABS } 173 \mathrm{mg} / \mathrm{cm}^{2} \\
\text { Front: Mylar }{ }^{\circledR} \text { window } 9 \mathrm{mg} / \mathrm{cm}^{2} \\
\text { Back: ABS } 173 \mathrm{mg} / \mathrm{cm}^{2} \\
\text { Front: ABS } 240 \mathrm{mg} / \mathrm{cm}^{2,} \text { Sn } 463 \mathrm{mg} / \mathrm{cm}^{2} \\
\text { Back: ABS } 173 \mathrm{mg} / \mathrm{cm}^{2}\end{array}$ \\
\hline $\begin{array}{l}\text { HCND } \\
\text { Beta Photon } \\
\text { Component } \\
\text { (8825BP card) } \\
\text { (8825 holder) }\end{array}$ & $\begin{array}{l}\text { 30xxxxx / } \\
030 \mathrm{xxxxx}\end{array}$ & $\begin{array}{l}\text { Element 1: TLD-700 }\left(100 \mathrm{mg} / \mathrm{cm}^{2}\right) \\
3.175 \text { x } 3.175 \text { x } 0.381 \mathrm{~mm}^{3} \\
\text { Element 2: TLD-700 }\left(100 \mathrm{mg} / \mathrm{cm}^{2}\right) \\
3.175 \text { x } 3.175 \times 0.381 \mathrm{~mm}^{3} \\
\text { Element 3: TLD-700 }\left(40 \mathrm{mg} / \mathrm{cm}^{2}\right) \\
3.175 \text { x 3.175 x } 0.152 \mathrm{~mm}^{3} \\
\text { Element 4: TLD-700 }\left(100 \mathrm{mg} / \mathrm{cm}^{2}\right) \\
3.175 \times 3.175 \times 0.381 \mathrm{~mm}^{3}\end{array}$ & $\begin{array}{l}\text { Front: ABS } 242 \mathrm{mg} / \mathrm{cm}^{2}, \mathrm{Cu} 91 \mathrm{mg} / \mathrm{cm}^{2} \\
\text { Back: ABS } 173 \mathrm{mg} / \mathrm{cm}^{2} \\
\text { Front: PTFE+ABS } 1000 \mathrm{mg} / \mathrm{cm}^{2} \\
\text { Back: ABS } 173 \mathrm{mg} / \mathrm{cm}^{2} \\
\text { Front: Mylar }{ }^{\circledR} \text { window } 9 \mathrm{mg} / \mathrm{cm}^{2} \\
\text { Back: ABS } 173 \mathrm{mg} / \mathrm{cm}^{2} \\
\text { Front: ABS } 240 \mathrm{mg} / \mathrm{cm}^{2,} \text { Sn } 463 \mathrm{mg} / \mathrm{cm}^{2} \\
\text { Back: ABS } 173 \mathrm{mg} / \mathrm{cm}^{2}\end{array}$ \\
\hline $\begin{array}{l}\text { HCND } \\
\text { Neutron } \\
\text { Component } \\
\text { (8816 card) } \\
\text { (8816 holder) }\end{array}$ & $\begin{array}{l}40 \mathrm{xxxxx} / \\
040 \mathrm{xxxxx}\end{array}$ & $\begin{array}{l}\text { Element 1: TLD-700 }\left(100 \mathrm{mg} / \mathrm{cm}^{2}\right) \\
3.175 \text { x } 3.175 \text { x } 0.381 \mathrm{~mm}^{3} \\
\text { Element 2: TLD-600 }\left(100 \mathrm{mg} / \mathrm{cm}^{2}\right) \\
3.175 \text { x } 3.175 \times 0.381 \mathrm{~mm}^{3} \\
\text { Element 3: TLD-600 }\left(100 \mathrm{mg} / \mathrm{cm}^{2}\right) \\
3.175 \text { x 3.175 x } 0.381 \mathrm{~mm}^{3} \\
\text { Element 4: TLD-600 }\left(100 \mathrm{mg} / \mathrm{cm}^{2}\right) \\
3.175 \times 3.175 \times 0.381 \mathrm{~mm}^{3}\end{array}$ & $\begin{array}{l}\text { Front: Sn } 464 \mathrm{mg} / \mathrm{cm}^{2}+\text { ABS } 80 \mathrm{mg} / \mathrm{cm}^{2} \\
\text { Back: Sn } 464 \mathrm{mg} / \mathrm{cm}^{2}+\text { ABS } 80 \mathrm{mg} / \mathrm{cm}^{2} \\
\text { Front: Cd } 461 \mathrm{mg} / \mathrm{cm}^{2}+\text { ABS } 80 \mathrm{mg} / \mathrm{cm}^{2} \\
\text { Back: Sn } 464 \mathrm{mg} / \mathrm{cm}^{2}+\text { ABS } 80 \mathrm{mg} / \mathrm{cm}^{2} \\
\text { Front: Sn } 464 \mathrm{mg} / \mathrm{cm}^{2}+\text { ABS } 80 \mathrm{mg} / \mathrm{cm}^{2} \\
\text { Back: Cd } 461 \mathrm{mg} / \mathrm{cm}^{2}+\text { ABS } 80 \mathrm{mg} / \mathrm{cm}^{2} \\
\text { Front: Sn } 464 \mathrm{mg} / \mathrm{cm}^{2}+\text { ABS } 80 \mathrm{mg} / \mathrm{cm}^{2} \\
\text { Back: Sn } 464 \mathrm{mg} / \mathrm{cm}^{2}+\text { ABS } 80 \mathrm{mg} / \mathrm{cm}^{2}\end{array}$ \\
\hline $\begin{array}{l}\text { EXT-RAD Ring } \\
\text { Dosimeter } \\
\text { (XD740) }\end{array}$ & $\begin{array}{l}00001-29999 / \\
30000-99999\end{array}$ & $\begin{array}{l}\text { Element 1: TLD-700 }\left(40 \mathrm{mg} / \mathrm{cm}^{2}\right) \\
3.175 \text { x } 3.175 \text { x } 0.152 \mathrm{~mm}^{3}\end{array}$ & $\begin{array}{l}\text { Density thickness of ring window is } \\
\text { approximately } 7 \mathrm{mg} / \mathrm{cm}^{2} \text {. }\end{array}$ \\
\hline $\begin{array}{l}\text { Environmental } \\
\text { Dosimeter } \\
(8807)\end{array}$ & $\begin{array}{l}90 \mathrm{xxxxx} / \\
090 \mathrm{xxxxx}\end{array}$ & $\begin{array}{l}\text { Elements 1,2: TLD-200 }\left(240 \mathrm{mg} / \mathrm{cm}^{2}\right) \\
3.175 \text { x } 3.175 \text { x } 0.889 \mathrm{~mm}^{3} \\
\text { Elements 3,4: TLD-700 }\left(240 \mathrm{mg} / \mathrm{cm}^{2}\right) \\
3.175 \text { x } 3.175 \text { x } 0.889 \mathrm{~mm}^{3}\end{array}$ & $\begin{array}{l}\text { Front and Back: Ta }\left(422 \mathrm{mg} / \mathrm{cm}^{2}\right), \mathrm{Pb} \\
\left(58 \mathrm{mg} / \mathrm{cm}^{2}\right), 80 \mathrm{mg} / \mathrm{cm}^{2} \text { ABS plastic } \\
\text { Front and Back: } 80 \mathrm{mg} / \mathrm{cm}^{2} \text { ABS plastic }\end{array}$ \\
\hline $\begin{array}{l}\text { Nuclear Accident } \\
\text { Dosimeter }\end{array}$ & N/A & $\begin{array}{l}\text { Pairs of TLD-700 and TLD- } 600 \text { chips } \\
\text { are contained in outer and inner } \\
\text { dosimetry capsules. }\end{array}$ & Plastic capsules are used to contain chips. \\
\hline
\end{tabular}

a. Elements on 8825, 8816 and 8807 card types consist of a TLD chip encapsulated in a PTFE (i.e. Teflon ${ }^{\circledR}$ ) film having a density thickness of $8 \mathrm{mg} / \mathrm{cm}^{2}$. The XD740 chip has no such encapsulation.

b. $\mathrm{PTFE}=$ polytetrafluorethylene

c. ABS = acrylonitrile-butadiene-styrene plastic

d. All dimensions are nominal. Manufacturing tolerances are not implied by the number of figures given. 
Table 5.3 also describes the holder for each dosimeter type, each of which contains specific design features and/or filtration to control the types of radiation admitted to the respective dosimeter phosphors. Holders for whole body dosimeters and environmental dosimeters are black to minimize light penetration to the card. Typically, the personnel dosimeters are mounted on a strap and coupled to the DOE security credential in a fashion that prevents visual obstruction of the picture and name on the badge but orients the dosimeter with the front facing "out." The HCND consists of a beta-photon dosimeter and a neutron dosimeter mounted in a plastic holder (not shown in Table 5.3). The HCND with holder is described in Section 5.5. The environmental dosimeter is mounted vertically in air. Extremity dosimeters include the four element HSD used as a wrist dosimeter, and a single chip finger ring dosimeter. The HSD and HCND area dosimeters have the same specifications given for the HSD and HCND personnel dosimeters in Table 5.3. The nuclear accident dosimeter is available in two forms described in greater detail in Section $\mathbf{5 . 1 0}$.

\subsubsection{Generating Calibration Cards}

Before a TLD system can be calibrated, it is necessary to establish a set of reference cards that will be used for purpose of calibrating the reader and calibrating other cards. This set of cards is exposed and read together as a group. For each element position on the TLD card, the mean light output of all chips in the same position is determined and the output of individual chips in that position compared against this.

A sensitivity factor known as an element correction coefficient (ECC) is determined for each chip such that the response of the chip after correction with the ECC is equivalent to the mean response of all reference chips in the same position on the card. Thus the ECC corrected responses of all chips in the calibration set are theoretically the same for any given dose (Plato et. al. 1985, Moscovitch 1993, Tawil 1996).

\subsubsection{TLD Reader Calibration}

Calibration of each reader is accomplished by exposing a representative sample of reader calibration cards in air without a holder to a known amount of radiation (typically ${ }^{60} \mathrm{Co}$ ) and then processing on the reader. The cards used to calibrate the reader are typically exposed to $500 \mathrm{mR}$ and a reader calibration factor (RCF) expressed in units of $\mathrm{nC} / \mathrm{mR}$ is calculated for each of the four PMT channels according to the following relationships:

$$
\begin{gathered}
R C F_{\mathrm{i}}=\frac{\overline{Q_{\mathrm{i}}}}{X} \\
\bar{Q}_{\mathrm{i}}=\frac{\sum_{\mathrm{j}=1}^{\mathrm{k}} Q_{\mathrm{ij}} * E C C_{\mathrm{ij}}}{k}
\end{gathered}
$$


where: $\mathrm{RCF}_{\mathrm{i}}=$ reader calibration factor for $\mathrm{i}^{\text {th }} \mathrm{PMT}(\mathrm{nC} / \mathrm{mR})$

$\mathrm{X}=$ exposure value $(\mathrm{mR})$

$\mathrm{Q}_{\mathrm{ij}}=$ raw reading for chip i of card $\mathrm{j}(\mathrm{nC})$

$\mathrm{ECC}_{\mathrm{ij}}=$ element correction coefficient for chip $\mathrm{i}$ of card $\mathrm{j}$

$\mathrm{k}=$ number of calibration cards used.

The reader calibration cards used for this purpose have been previously calibrated, and the resulting ECC for each chip is applied to its raw reading to correct for variations in chip sensitivity. This approach allows for a more precise measure of PMT sensitivity to be obtained in the reader calibration process with fewer cards. Reader calibration is performed prior to, and as an independent process from, the read-out of personnel cards for dose determination. The RCFs obtained in the reader calibration process are then applied to the subsequent field and QC card readings on a real-time basis as the cards are read. When RCFs are applied, chip readings are reported from the reader in units of ${ }^{60} \mathrm{Co} \mathrm{mR}$ equivalent (in air).

\subsubsection{Card Calibration}

The ECC of a given TLD element is a measure of how the element responds to a source of radiation relative to the response of other similar elements in a reference population (calibration card set). The ECC for a given chip corrects that chip's sensitivity to the mean sensitivity of chips in the same position in the population of cards used to calibrate the reader. The process of card calibration entails calibrating the reader with a sample of reader calibration cards exposed to a known amount of radiation and applying the resulting RCFs to subsequent readings of cards being calibrated (which have been exposed to the same source). The ECC for each chip is determined according to the following relationship:

$$
E C C_{\mathrm{ij}}=\frac{R C F_{\mathrm{i}}}{Q_{\mathrm{ij}}} X
$$

where: $\quad$ ECC $_{\mathrm{ij}}=$ element correction coefficient for chip $\mathrm{i}$ on card $\mathrm{j}$

$\mathrm{RCF}_{\mathrm{i}}=$ reader calibration factor for $\mathrm{PMT}_{\mathrm{i}}(\mathrm{nC} / \mathrm{mR})$

$\mathrm{Q}_{\mathrm{ij}}=$ raw reading from chip $\mathrm{i}$ on card $\mathrm{j}(\mathrm{nC})$

$\mathrm{X}=$ delivered ${ }^{60}$ Co exposure value $(\mathrm{mR})$.

The ECCs are determined for a group of cards by annealing all cards, including "calibration cards," as a group (i.e. at the same time), followed by irradiation as a group and readout on the same day. Because of the demonstrated good reproducibility of response for TL elements in HEDP cards, only one exposure and readout are necessary to accurately determine the initial ECC for an element. ${ }^{\text {(a) }}$ Subsequent calibrations of the card entail a comparison of the old ECC with the new ECC and rejections of the new ECC if it is not within $20 \%$ of

(a) “Model 8800 Automatic TLD Card Reader with TLD-REMS User's Manual” Publication No. 8800-R-U-1188-001, release date November 15, 1992, Bicron (copy retained in HEDP files).

W. V. Baumgartner, “Comparison of ECCs Obtained From Calibration Cards,” August 5, 1993, letter to HEDP file. 
the old. Cards are re-calibrated on approximately a five $(5 \pm 1)$ year cycle. ${ }^{\text {(a) }}$ Cards with ECCs six or more years old are prevented from being issued by the External Dosimetry Data Management System.

\subsubsection{Dosimeter Calibration}

HEDP calibrates TLD readers with cards exposed in air to a local source $\left({ }^{60} \mathrm{Co}\right)$. When field cards used in dosimeter holders are read on a reader calibrated in this manner, the readings are given in units of $\mathrm{mR}\left({ }^{60} \mathrm{Co} \mathrm{mR}\right.$ equivalent). It is necessary for the dose algorithm to convert these readings to the equivalent readings that would be obtained with reference geometry and reference source (e.g. in holder, on-phantom, at 1 meter, to a reference source described in the DOELAP standard). For the HEDP system, dosimeter calibration consists of determining the relationship between each chip's response to ${ }^{60} \mathrm{Co}$ in air without a holder, to its response to ${ }^{137} \mathrm{Cs}$ when irradiated in a holder, on a phantom in reference geometry. The resulting factor is called the ${ }^{137} \mathrm{Cs}$ relative response factor (RRF) and is expressed in units of $\mathrm{mR} / \mathrm{rem}$ for each chip position. The RRF is a function of the chip thickness, the radiation types and energies for reference and local geometry, and the composition and thickness of filtration over the chip for the reference and local geometry. In the HEDP system, conditions of charged particle equilibrium (CPE) are satisfied for both the local and reference geometries. RRFs vary with dosimeter type and chip position.

For each dosimeter design, the RRF is determined by exposing a set of cards to ${ }^{60} \mathrm{Co}$ in air and a set of cards to the ${ }^{137} \mathrm{Cs}$ source on-phantom, and then reading the cards together in a single processing on a stable reader with ECCs applied. For each chip position, the ratio of the average response to the ${ }^{137} \mathrm{Cs}$ source on phantom (nC/rem) to the average response to the ${ }^{60} \mathrm{Co}$ source in air $(\mathrm{nC} / \mathrm{mR})$ is calculated. This ratio (mR/rem) is the ${ }^{137} \mathrm{Cs} \mathrm{RRF}$ for that chip position in that dosimeter design. Dividing the calibrated reading ( $\mathrm{mR}$ ) for a given chip by the RRF for that position provides a ${ }^{137} \mathrm{Cs}$ rem-equivalent reading for that chip. This is the same reading that would have been obtained if the reader had been calibrated directly with cards exposed in holders and on-phantom to the calibration standard. Establishing and using RRFs allows for accurate system calibration without the need for costly routine irradiations with a calibration standard. It also allows flexibility to use a variety of local sources as backup sources for calibration and still obtain equally valid dose results. For the HEDP dosimetry system, RRFs have been determined for a primary ${ }^{60}$ Co source and irradiation jig, and a backup ${ }^{90} \mathrm{Sr}$ card irradiator located in one of the TLD readers. $^{\text {(b) }}$ RRFs have been determined for each chip position in each of the dosimeter types used at Hanford.

(a) B. A. Rathbone, “ECC Stability Study,” June 8, 1998, letter to HEDP file.

(b) B. A. Rathbone, "Re-evaluation of RRF Data for HSD and HCND," October 6, 1999, letter to HEDP file. 


\subsubsection{Calibrated Element Readings}

When TLD cards are read, the reader applies ECCs and RCFs to the raw light output expressed as charge collected on the PMT (nC), to obtain calibrated element readings as follows:

$$
X_{i}=Q_{i} * E C C_{i} / R C F_{i}
$$

where $\mathrm{X}_{\mathrm{i}}=$ calibrated reading for element $\mathrm{i}(\mathrm{mR})$

$\mathrm{Q}_{\mathrm{i}}=$ raw reading from element $\mathrm{i}(\mathrm{nC})$

$\mathrm{ECC}_{\mathrm{i}}=$ element correction coefficient for element $\mathrm{i}$

$\mathrm{RCF}_{\mathrm{i}}=$ reader calibration factor for position $\mathrm{i}(\mathrm{nC} / \mathrm{mR})$.

\subsubsection{Adjusted Element Readings}

When TLD card readings are processed on the External Dosimetry Data Management System, environmental background is subtracted, then supralinearity and fade corrections are made to the calibrated element readings to obtain “adjusted” element readings in ${ }^{137} \mathrm{Cs}$ mrem equivalent, as follows:

$$
\begin{aligned}
\text { Xnet }_{i} & =\left(X_{i}-E_{i}\right) \\
D_{i} & =\text { Xnet }_{i} /\left(R R F_{i} * F_{i} * S_{i}\right)
\end{aligned}
$$

where $\quad D_{i}=$ adjusted element reading for element $\mathrm{i}\left({ }^{137} \mathrm{Cs}\right.$ mrem equivalent)

$\mathrm{X}_{\mathrm{i}}=$ calibrated element reading for element i ( ${ }^{60} \mathrm{Co} \mathrm{mR}$ equivalent)

$\mathrm{E}_{\mathrm{i}}=$ estimated background signal for element i ( ${ }^{60} \mathrm{Co} \mathrm{mR}$ equivalent)

$\mathrm{RRF}_{\mathrm{i}}={ }^{137} \mathrm{Cs}$ relative response factor for element $\mathrm{i}(\mathrm{mR} / \mathrm{mrem})$

$F_{i}=$ fade factor for element $\mathrm{i}$

$\mathrm{S}_{\mathrm{i}}=$ supralinearity factor for element $\mathrm{i}$.

Correction of individual element readings for supralinearity and fade prior to use by the algorithm allows the algorithm to provide valid results based on element ratios over a wide range of doses and wear periods. Because supralinearity and fading vary depending on the radiation type (McKeever, Moscovitch, and Townsend 1995; Horowitz;1984) the values of $S_{i}$ and $F_{i}$ in Equation 5.6 are weighted averages calculated on the basis of the estimated fraction of the TL signal due to neutron radiation and the fraction due to beta-gamma radiation, as described below.

\subsubsection{Models for Environmental Background}

The total background that is subtracted from each calibrated chip reading is calculated from an empirically derived background function $\mathrm{E}_{\mathrm{i}}$ for each chip position i in each dosimeter type as follows:

$$
\mathrm{E}_{\mathrm{i}}=\mathrm{G}_{\mathrm{i}} * \mathrm{FD}+\mathrm{B}_{\mathrm{i}}
$$

where: $\mathrm{G}_{\mathrm{i}} \quad=\quad$ background growth rate $(\mathrm{mR} / \mathrm{d})$

$$
\begin{array}{ll}
\mathrm{FD} \quad= & \begin{array}{l}
\text { field cycle days (days between previous and current } \\
\text { processing date for the card) }
\end{array} \\
\mathrm{B}_{\mathrm{i}} \quad=\quad \begin{array}{l}
\text { intrinsic background signal (mR) }
\end{array}
\end{array}
$$


The slope and intercept for the environmental background function $E_{\mathrm{i}}$ vary by chip position and dosimeter type. The slope and intercept measured for the Hanford site are given in Table 5.4.

Table 5.4 Parameters for Background Functions

\begin{tabular}{||c|c|c|c||}
\hline \multirow{2}{*}{ Parameter } & HSD & \multicolumn{2}{|c|}{ HCND } \\
\cline { 2 - 4 } & $8825 \mathrm{BPN}$ & $8825 \mathrm{BP}$ & $8816 \mathrm{~N}$ \\
\hline \hline $\mathrm{G}_{1}$ & 0.145 & 0.145 & 0.163 \\
\hline $\mathrm{G}_{2}$ & 0.140 & 0.140 & 0.171 \\
\hline $\mathrm{G}_{3}$ & 0.148 & 0.148 & 0.169 \\
\hline $\mathrm{G}_{4}$ & 0.195 & 0.145 & 0.192 \\
\hline $\mathrm{B}_{1}$ & 1.42 & 1.42 & 1.89 \\
\hline $\mathrm{B}_{2}$ & 1.32 & 1.32 & 2.09 \\
\hline $\mathrm{B}_{3}$ & 2.95 & 2.95 & 2.23 \\
\hline $\mathrm{B}_{4}$ & 1.97 & 1.37 & 2.50 \\
\hline
\end{tabular}

Slightly different values have been established for use at sites other than Hanford. It should be noted that neither $B_{i}$ nor $G_{i}$ have been corrected for fade. Since $E_{i}$ is therefore not corrected for fade, it is subtracted directly from $X_{i}$ which is also not corrected for fade.

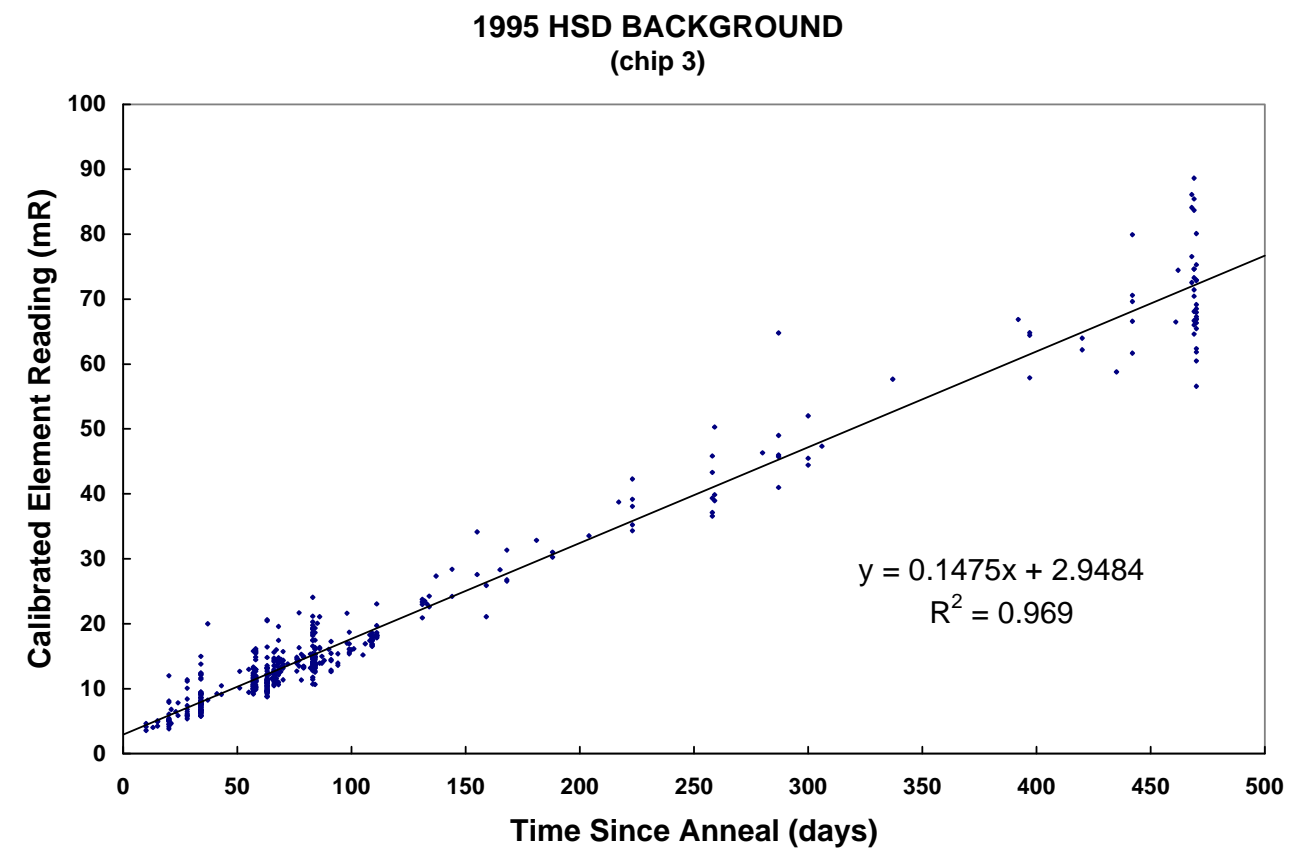

Figure 5.3 Buildup of Environmental Background Signal in HSD Shallow Dose Element 


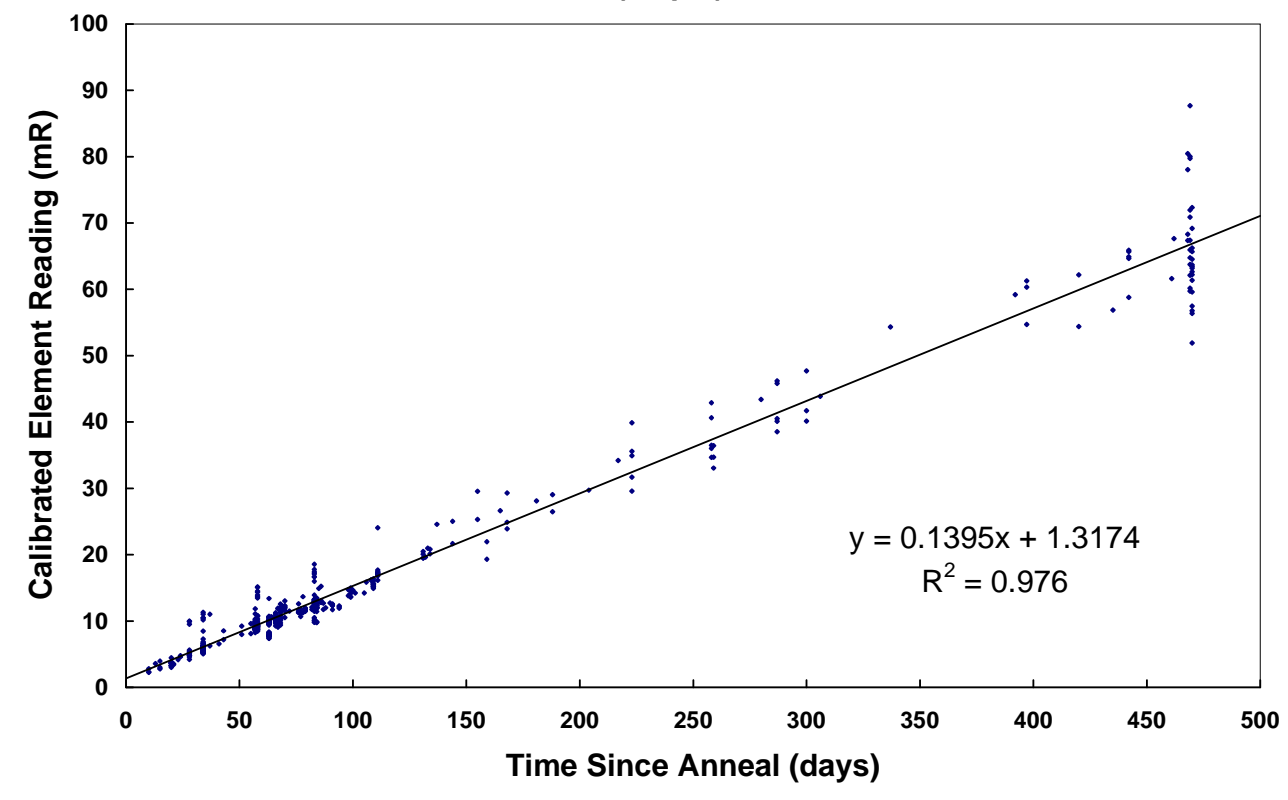

Figure 5.4 Buildup of Environmental Background Signal in HSD Deep Dose Element

The background functions established for Hanford ${ }^{\text {(a) }}$ were determined from a least squares fit of a line to calibrated element readings from dosimeters that were stored at the 318 building and other Hanford facilities for varying periods up to 470 days. The storage locations were generally believed to be representative of natural background radiation levels at Hanford. Plots of the shallow dose element (chip 3) data and deep dose element (chip 2) data for the HSD are shown in Figure 5.3 and Figure 5.4 respectively. The $\mathrm{mR} / \mathrm{d}$ values used in all background functions are less than the yearly and five year average radiation levels measured at offsite locations (perimeter, community and distant locations) as part of the Hanford Environmental Surveillance Program (Antonio 1999, Antonio 2002). The background functions used for personnel and area dosimeters are therefore expected to provide a conservative measure of occupational dose.

Radiation measurement data from environmental surveillance reports indicate that the natural radiation background at Hanford and in nearby communities has varied by less than 5\% from year to year over a seven year period from 1995-2002 Although in some regions seasonal variations can be as large as $25 \%$ due to changes in soil moisture and snow cover and as large as $10 \%$ due to changes in cosmic radiation (NCRP 1987),the data for Hanford indicate that quarterly variations during the time period from $1995-2002$ are less than $10 \%$. The above data suggest that personnel and area dosimeter background functions should not need to be changed from year to year. Analysis of HEDP annual audit dosimeter data for the same time period supports this conclusion. An analysis of potential errors in dose results that arise from use of a pre-determined background function indicates that relative to the DOE monitoring threshold of $100 \mathrm{mrem} / \mathrm{y}$, the

(a) B. A. Rathbone, "Re-evaluation of Background Functions for HSD and HCND Dosimeters," October 3, 1999, letter to HEDP file. 
standard uncertainty of $\pm 20 \mathrm{mrem} / \mathrm{y}$ in recorded dose is acceptable.

\subsubsection{Models for Fading}

Fade corrections for each chip are based on empirical models of post irradiation fading for TLD 600 and 700 developed for routine dosimeter annealing and readout protocols at Hanford. ${ }^{\text {(a) }}$ For each model, a non-linear least squares regression analysis was performed to fit a two compartment model to dosimeter response data. Because fading of neutron signal in TLD 600 is significantly more pronounced than fading of beta-gamma signal in TLD 600, (Johnson and Luersen, 1980; Horowitz, 1984; Doremus and Higgins, 1994; McKeever, Moscovitch and Townsend, 1995) separate models were necessary for beta-gamma and neutron fading in TLD 600. In addition, a model for beta-gamma fading in TLD 700 was developed. All three models have the form given in Equation 5.8.

$$
F(t)=R(t) / R_{0}=a e^{\left(-\lambda_{1} t\right)}+(1-a) e^{\left(-\lambda_{2} t\right)} \quad \text { (eqn. 5.8) }
$$

where: $\quad t$ is the time since irradiation (days)

$\mathrm{R}(\mathrm{t})$ is the net chip response $(\mathrm{mR})$ at time $\mathrm{t}$

$\mathrm{R}_{0}$ is the net chip response $(\mathrm{mR})$ at time $\mathrm{t}=0$

$\mathrm{a}$ is the weighting factor for the short half-life compartment

$\lambda_{1}$ is the decay constant for the short half-life compartment

$\lambda_{2}$ is the decay constant for the long half-life compartment

For routine dosimetry, when the time since irradiation is generally not known, one half of the time between previous and current processing is used for t. The parameters to be used in the model for each phosphor and radiation type are shown in Table 5.5. The three models are shown graphically in Figure 5.5.

Table 5.5 Parameters for use in Post Irradiation Fade Models

\begin{tabular}{||l|l|l|l||}
\hline \hline Parameter & TLD 700 $\beta-\gamma$ & TLD 600 $\beta-\gamma$ & TLD 600 Neutron \\
\hline $\mathrm{a}$ & 0.0530 & 0.0851 & 0.1550 \\
\hline$\lambda_{1}$ & $0.0179 \mathrm{~d}^{-1}$ & $0.0135 \mathrm{~d}^{-1}$ & $0.0286 \mathrm{~d}^{-1}$ \\
\hline$\lambda_{2}$ & $0.000231 \mathrm{~d}^{-1}$ & $0.000135 \mathrm{~d}^{-1}$ & $0.000367 \mathrm{~d}^{-1}$ \\
\hline \hline
\end{tabular}

The fade models above were developed based on experiments in which single acute exposures are used, the pre-irradiation time interval is held small and constant, and only the post-irradiation interval is varied. Thus the model predicts post-irradiation fading. Models of this type are limited in actual use because the exposure time is seldom known, and exposure in the field is often chronic rather than acute. A comparison of fading from chronic exposure and fading from single

acute exposure, was made in the referenced neutron fading study. The comparison

(a) B. A. Rathbone, "Re-evaluation of Post-irradiation Fading of Beta-Gamma Dose in TLD 600 and TLD 700, March 20, 2000, letter to HEDP file.

B. A. Rathbone, "Post Irradiation Fading of Neutron Signal in TLD 600”, March 23, 2000, letter to HEDP file. 
showed that for neutron exposure in TLD 600, the default assumption for $\mathrm{t}$ [ $\mathrm{t}=$ (T2-T1)/2 where T2 and T1 are readout date and anneal dates respectively] in the post irradiation fade model, results in a slight over estimate $(\mathrm{F}=0.78)$ of the actual observed fading from chronic exposure $(\mathrm{F}=0.82)$ by about $5 \%$ for an annual dosimeter. Because the magnitude of fading with beta-gamma exposure is smaller, the differences between model predictions and observed fading with chronic exposure are correspondingly smaller. The errors in fade correction incurred by assuming a mid cycle exposure for an actual exposure occurring at the beginning or end of a use cycle can be readily calculated and are shown Figures 5.6 and 5.7. Other errors involve uncertainty in the loss of sensitivity with time before irradiation (pre-irradiation fading). For a 407 day pre-irradiation fade interval, the loss of sensitivity for beta-gamma dose has been estimated to be about $6 \%$. These and other sources of uncertainty in fade corrections are assessed in greater detail in an HEDP internal study. ${ }^{\text {(a) }}$

The fading corrections for TLD 600 must be applied in a weighted manner based on the estimated fractions of the background corrected TL signal attributable to beta-gamma radiation and to neutron radiation as described in Section 5.3.6.4.

\section{Post Irradiation Fading in TLD 600 and TLD 700}

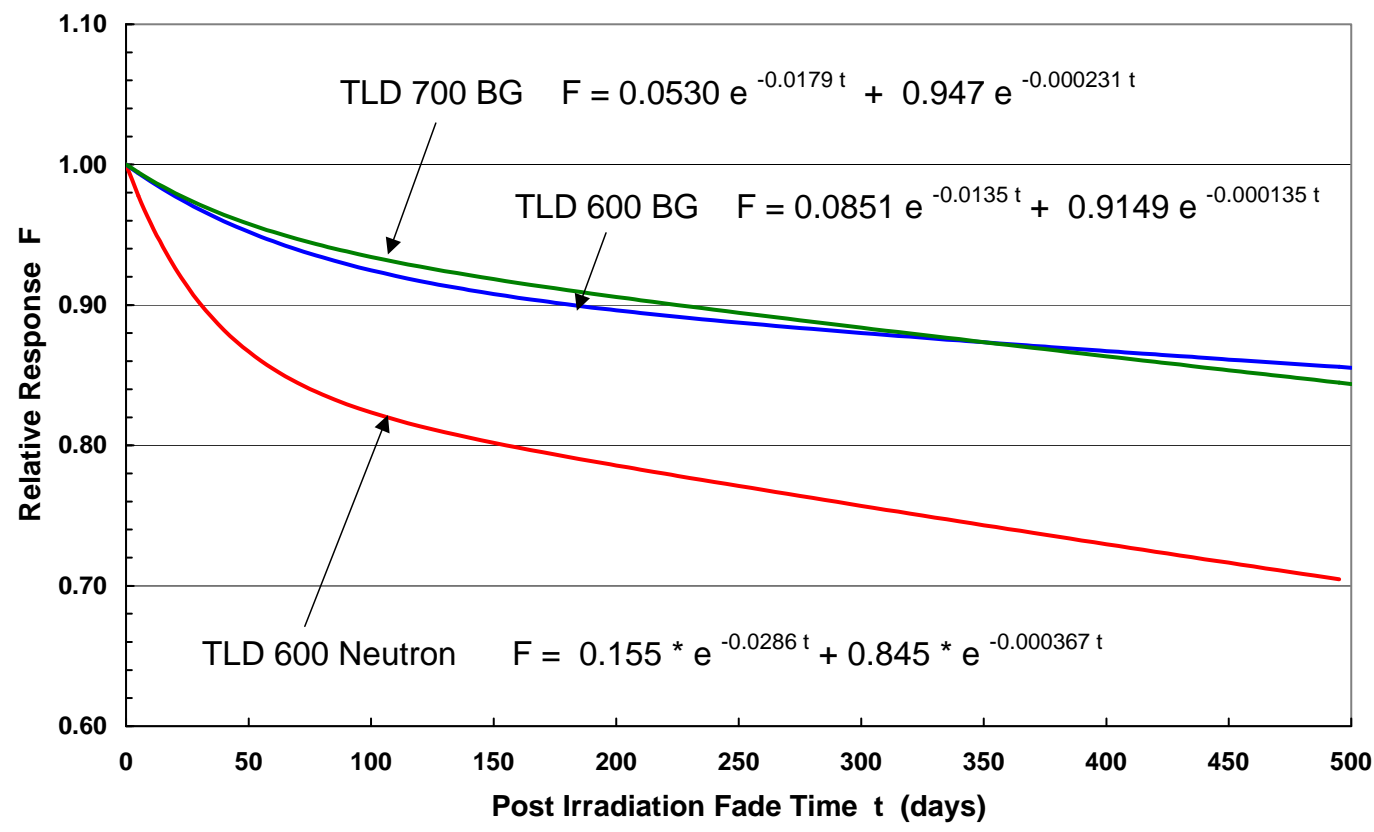

Figure 5.5 Post Irradiation Fade Functions Used for Hanford Dosimetry Materials

(a) B. A. Rathbone, "Potential Errors from Using a Post-Irradiation Model of Fading in TLD 600 and TLD 700", March 24, 2000, letter to HEDP file. 


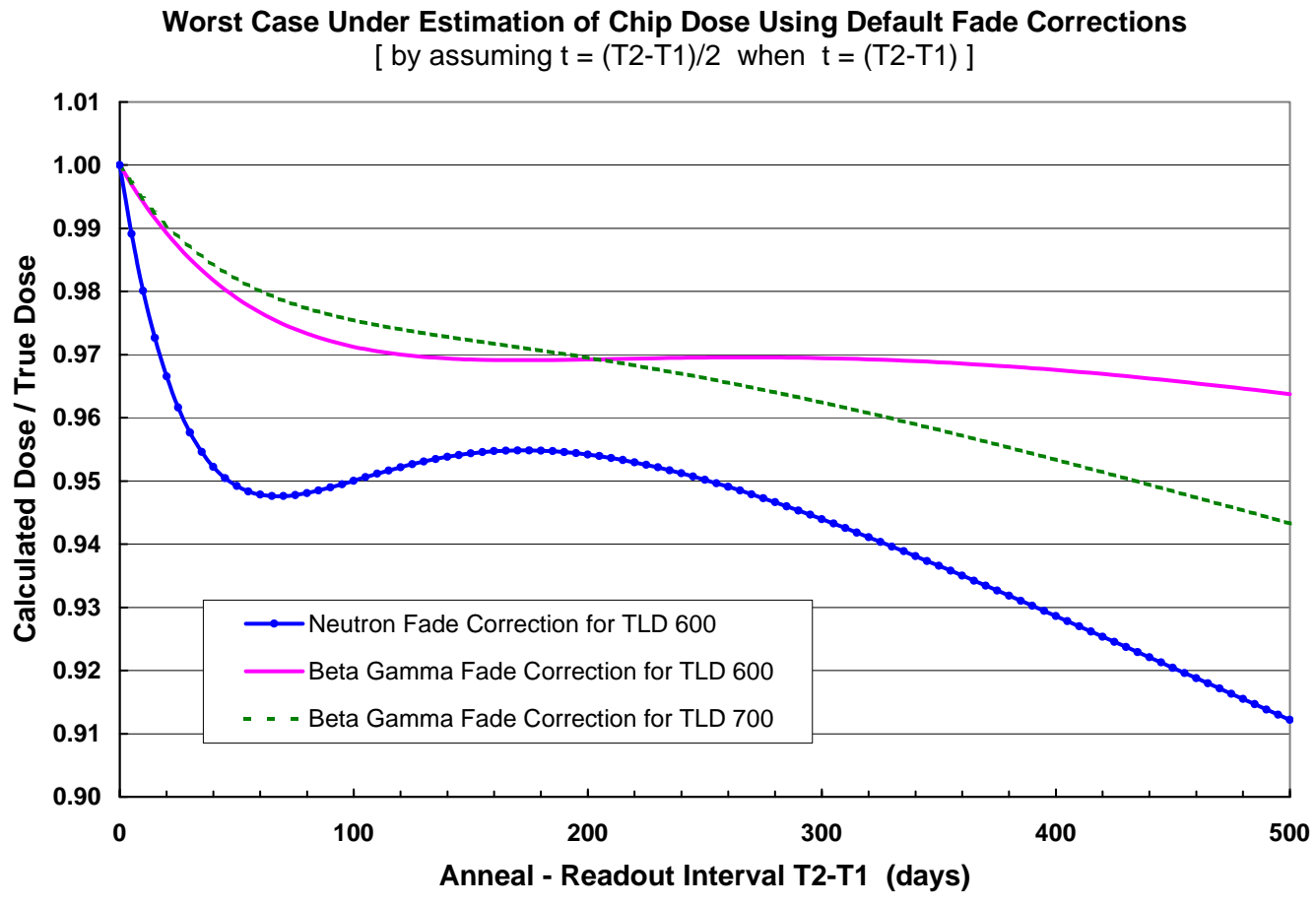

Figure 5.6 Error in default fade correction when entire exposure occurs on first day of use cycle.

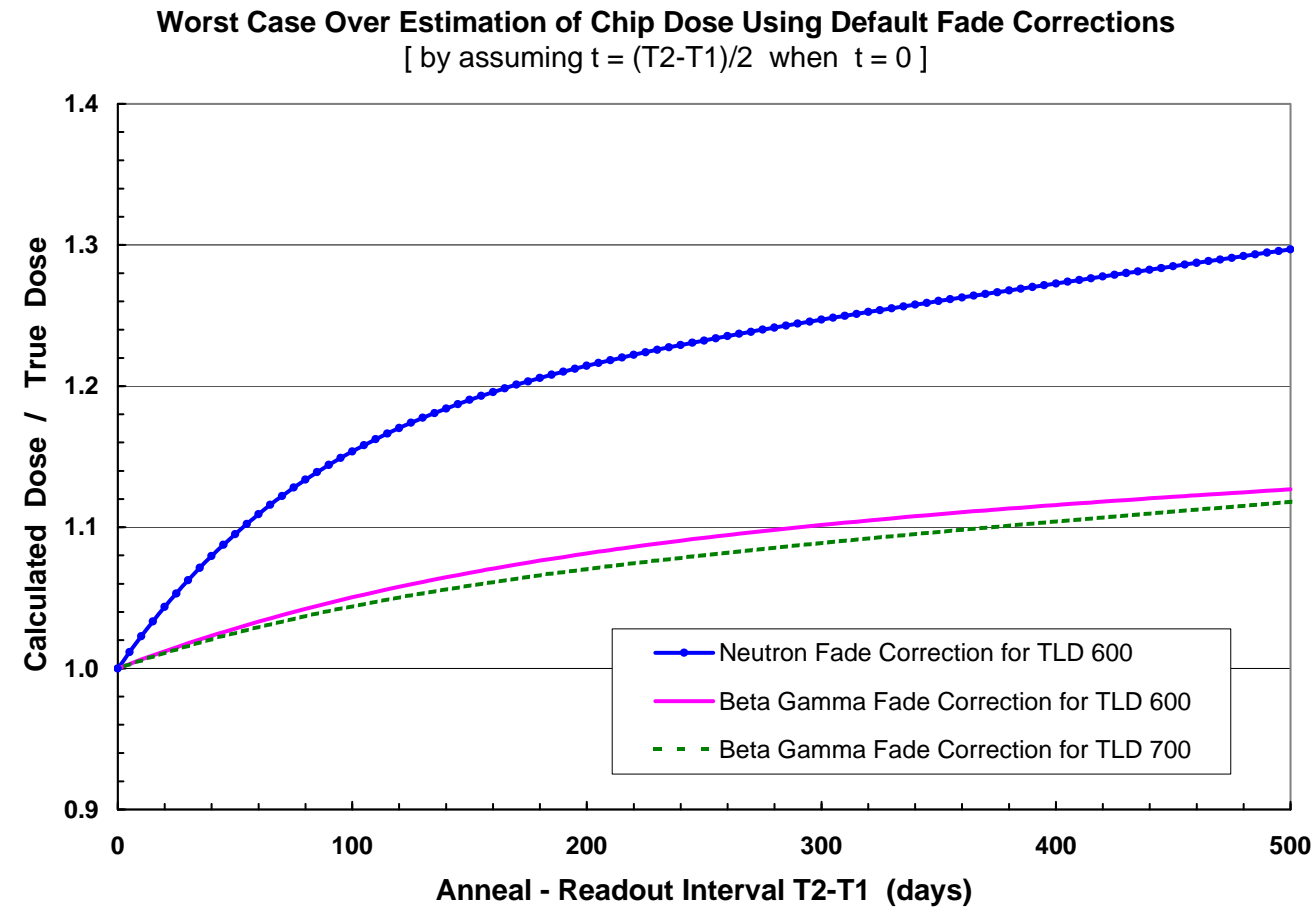

Figure 5.7 Error in default fade correction when entire exposure occurs on last day of use cycle. 


\subsubsection{Models for Supralinearity}

The empirically derived model ${ }^{(a)}$ for supralinearity of beta-gamma signal in TLD 600 and TLD 700 is as follows:

Sgamma $=1+3.411 E-7 *$ Xnet

where:

$\begin{array}{lll}\text { Sgamma } & = & \text { net reading of chip }(\mathrm{mR}) / \text { given exposure }(\mathrm{mR}) \\ \mathrm{Xnet} & =\mathrm{X}-\mathrm{E}=\text { background corrected chip reading }(\mathrm{mR}) \\ \mathrm{E} & = & \text { estimated intrinsic }+ \text { environmental background signal } \\ & & \text { on chip }(\mathrm{mR}) \\ \mathrm{X} & = & \text { calibrated chip reading for chip i }\left({ }^{60} \mathrm{Co} \mathrm{mR} \text { equivalent }\right)\end{array}$

The data used to develop the model and a least squares fit of the model to the data are shown in Figure 5.8.

TLD 600/700 SUPRALINEARITY IN THE HSD

(all chips)

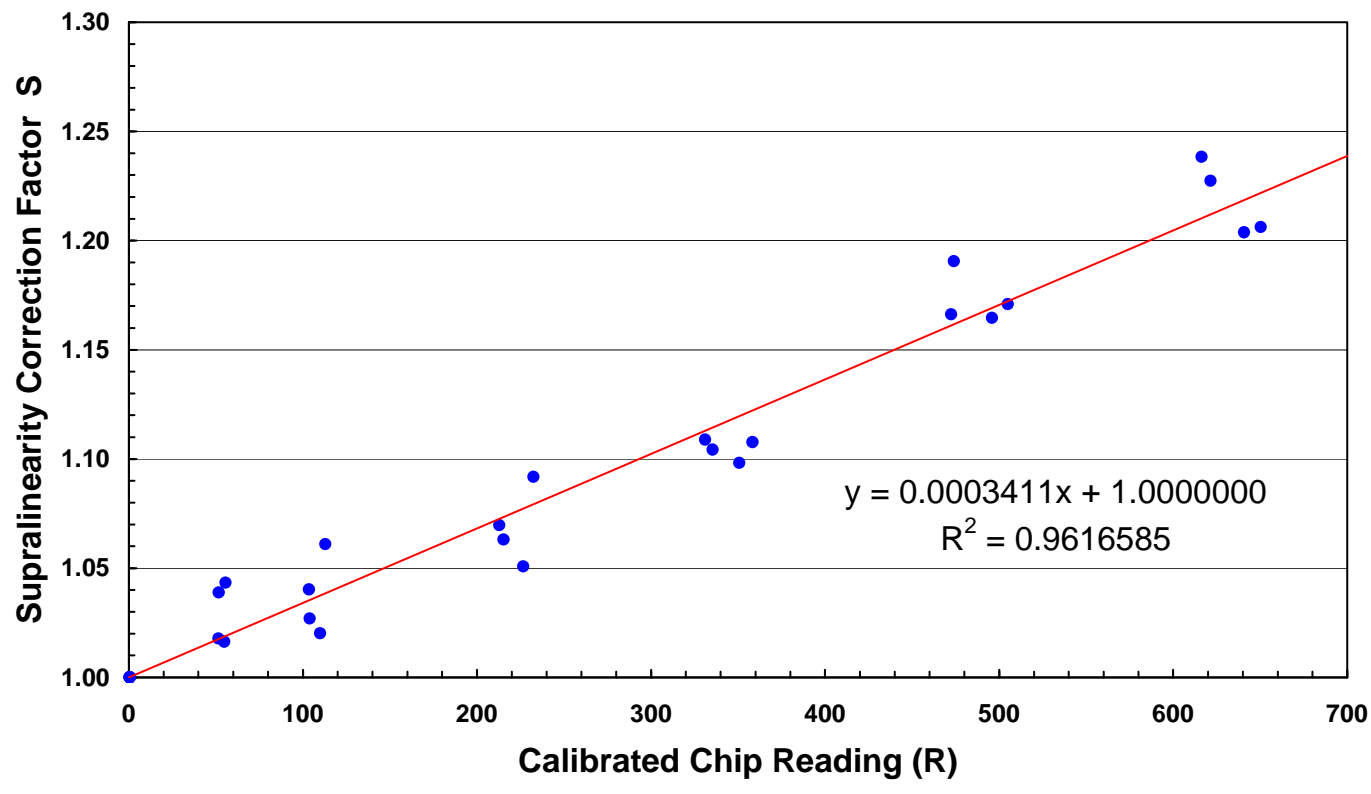

Figure 5.8. Supralinearity Correction for Beta-Gamma Signal in TLD 600 and 700.

(a) B. A. Rathbone, “Supralinearity Corrections for TLD 600 and 700,” April 3, 2000, letter to HEDP file. 
This supralinearity model is not appropriate for high linear energy transfer (LET) radiations. In particular, it is not valid for neutron induced TL on the TLD 600 chip. The TL output from TLD 600 when exposed to thermal neutrons is due primarily to dose deposited in the chip from alpha particles and recoil tritons from the ${ }^{6} \mathrm{Li}(\mathrm{n}, \alpha)^{3} \mathrm{H}$ capture reaction. The scientific literature on TLD suggests that the response of TLD 600 to thermal neutron radiation shows considerably less supralinearity than the response of either TLD 600 or TLD 700 to high energy gamma radiation (Cameron, Suntharalingam, and Kenney 1968; Douglas 1981; Horowitz 1984). TLD 600 shows a maximum over response to neutrons between a factor of 1.2 and 1.5 at doses between 10,000 and 50,000 rad as reported by various authors (Horowitz 1984). Compared to this, the maximum over response of TLD 600 and 700 to high energy gamma radiation is between a factor of 3 and 4 in the same dose range. The over response to neutrons is essentially non-existent at roentgen equivalent response levels less than 10,000 $\mathrm{R}$ (Douglas 1981). Therefore, a separate supralinearity model is used to correct the neutron portion of the TL signal. Based on data from Douglas (1981), the supralinearity correction is (conservatively) assumed to be equal to 1.0 for element readings up to $1000 \mathrm{R}$. The model for supralinearity of neutron signal in TLD 600 is as follows:

Sneutron $=1$

The supralinearity corrections for TLD 600 must be applied in a weighted manner based on the estimated fractions of the background corrected TL signal attributable to beta-gamma radiation and to neutron radiation as described below. This assumes that the two types of supralinearity act independently.

\subsubsection{Calculation of Weighted Fading and Supralinearity Corrections $F_{i}$ and $S_{i}$}

Weighted corrections for fading $F_{i}$ and supralinearity $S_{i}$ are calculated for each chip on each dosimeter based on the estimated fraction of TL signal due to betagamma radiation ( $\left.\mathrm{W}_{\text {gamma }}\right)$ as opposed to neutron radiation $\left(\mathrm{W}_{\text {neutron }}\right)$, and the fading and supralinearity corrections appropriate to each type of radiation. For TLD 600 elements, the estimated fraction of the TL signal due to neutron radiation is determined by comparing the TLD 600 element reading to that of a TLD 700 element reading (beta-gamma sensitive only) under similar filtration. For TLD 700 elements, the signal is assumed to be due entirely to beta-gamma radiation.

Do: $\mathrm{i}=1-4$

If: Xnet $_{\mathrm{i}}>0$,

$$
\begin{aligned}
& \text { If:card type }=0,9,10 \text {, or 60, and } \quad \mathrm{i}=4 \quad \text { (TLD } 600 \text { in } 8825 \text { card) } \\
& \text { If:Xnet }{ }_{\mathrm{i}}>\text { Xnet }_{2} \quad \text { (neutrons present) } \\
& \text { Wgamma }_{\mathrm{i}}=\text { Xnet }_{2} / \text { Xnet }_{\mathrm{i}} \\
& \text { Wneutron }_{\mathrm{i}}=1-\text { Wgamma }_{\mathrm{i}} \\
& \text { Fgamma }_{\mathrm{i}}=0.0851 \exp (-0.0135 \mathrm{t})+0.9149 \exp (-0.000135 \mathrm{t}) \\
& \text { Fneutron }_{\mathrm{i}}=0.1550 \exp (-0.0286 \mathrm{t})+0.8450 \exp (-0.000367 \mathrm{t})
\end{aligned}
$$




$$
\begin{array}{ll}
\text { Sgamma }_{\mathrm{i}} & =1+3.411 \mathrm{E}-7 * \text { Xnet }_{2} \\
\text { Sneutron }_{\mathrm{i}} & =1 \\
\mathrm{~F}_{\mathrm{i}} & =\left(\text { Wgamma }_{\mathrm{i}} * \text { Fgamma }_{\mathrm{i}}\right)+\left(\text { Wneutron }_{\mathrm{i}} * \text { Fneutron }_{\mathrm{i}}\right) \\
\mathrm{S}_{\mathrm{i}} & =\left(\text { Wgamma }_{\mathrm{i}} * \text { Sgamma }_{\mathrm{i}}\right)+\left(\text { Wneutron }_{\mathrm{i}} * \text { Sneutron }_{\mathrm{i}}\right)
\end{array}
$$

Else: (neutrons not present)

$$
\begin{array}{ll}
\mathrm{F}_{\mathrm{i}} & =0.0851 \exp (-0.0135 \mathrm{t})+0.9149 \exp (-0.000135 \mathrm{t}) \\
\mathrm{S}_{\mathrm{i}} & =1+3.411 \mathrm{E}-7 * \text { Xnet }_{\mathrm{i}}
\end{array}
$$

End If

Elseif: $\quad$ card type $=40,46,49$ and $\mathrm{i}=2,3$ or 4 (TLD 600 in 8816 card)

$$
\begin{aligned}
\text { If: }^{\text {Xnet }}{ }_{\mathrm{i}}>\text { Xnet }_{1} & \text { (neutrons present) } \\
\text { Wgamma }_{\mathrm{i}} & =\text { Xnet }_{1} / \text { Xnet }_{\mathrm{i}} \\
\text { Wneutron }_{\mathrm{i}} & =1-\text { Wgamma }_{\mathrm{i}} \\
\text { Fgamma }_{\mathrm{i}} & =0.0851 \exp (-0.0135 \mathrm{t})+0.9149 \exp (-0.000135 \mathrm{t}) \\
\text { Fneutron }_{\mathrm{i}} & =0.1550 \exp (-0.0286 \mathrm{t})+0.8450 \exp (-0.000367 \mathrm{t}) \\
\text { Sgamma }_{\mathrm{i}} & =1+3.411 \mathrm{E}-7 * \text { Xnet }_{1} \\
\text { Sneutron }_{\mathrm{i}} & =1 \\
\mathrm{~F}_{\mathrm{i}} & =\left(\text { Wgamma }_{\mathrm{i}} * \text { Fgamma }_{\mathrm{i}}\right)+\left(\text { Wheutron }_{\mathrm{i}} * \text { Fneutron }_{\mathrm{i}}\right) \\
\mathrm{S}_{\mathrm{i}} & \left.=\left(\text { Wgamma }_{\mathrm{i}} * \text { Sgamma }_{\mathrm{i}}\right)+\text { Wneutron }_{\mathrm{i}} * \text { Sneutron }_{\mathrm{i}}\right)
\end{aligned}
$$

Else: (neutrons not present)

End If

$$
\begin{array}{ll}
\mathrm{F}_{\mathrm{i}} & =0.0851 \exp (-0.0135 \mathrm{t})+0.9149 \exp (-0.000135 \mathrm{t}) \\
\mathrm{S}_{\mathrm{i}} & =1+3.411 \mathrm{E}-7 * \text { Xnet }_{\mathrm{i}}
\end{array}
$$

Else: (TLD 700)

$$
\begin{aligned}
& \mathrm{F}_{\mathrm{i}}=0.0530 \exp (-0.0179 \mathrm{t})+0.9470 \exp (-0.000231 \mathrm{t}) \\
& \mathrm{S}_{\mathrm{i}}=1+3.411 \mathrm{E}-7 * \text { Xnet }_{\mathrm{i}}
\end{aligned}
$$

End If (card type =)

End If $\left(\right.$ Xnet $\left._{\mathrm{i}}>0\right)$

End Do $(i=1-4)$ 


\subsubsection{Dose Algorithms}

Each type of dosimeter has an algorithm that calculates dose based on the adjusted element readings of the dosimeter. The specific algorithms used for Hanford dosimeters are documented in HEDP files in sufficient detail to allow hand calculation of dose. ${ }^{\text {(a) }}$ The dose quantities calculated for personnel dosimeters are operational quantities generally accepted for use in demonstrating compliance with the protection quantities used by DOE in occupational radiation protection requirements (DOE 2007). Dose algorithms for HEDP personnel dosimeter have been developed to calculate the following operational quantities (personal dose equivalent):

$$
\begin{aligned}
& \mathrm{H}_{\mathrm{p}}(0.07)_{\beta \gamma} \\
& \mathrm{H}_{\mathrm{p}}(3)_{\beta \gamma} \\
& \mathrm{H}_{\mathrm{p}}(10)_{\gamma} \\
& \mathrm{H}_{\mathrm{p}}(10)_{\eta},
\end{aligned}
$$

Because HEDP dosimeters are capable of measuring and reporting only the betagamma component of $\mathrm{H}_{\mathrm{p}}(0.07)$ and $\mathrm{H}_{\mathrm{p}}(3)$, the $\beta \gamma$ subscript is generally understood and omitted when discussing quantities measured and reported by HEDP dosimeters. Operational quantities reported by HEDP dosimeters and their use in demonstrating compliance with DOE limits expressed as protection quantities, are described in Chapter 4.

Chapter 6 of DOE G 441.1-1C Radiation Protection Programs Guide (DOE 2008a) lists the depths of interest both in terms of linear distance and their (implied) density thickness equivalent for soft tissue. In other words, per DOE guidance, it may be assumed that $1 \mathrm{~cm}=1000 \mathrm{mg} / \mathrm{cm}^{2}, 0.3 \mathrm{~cm}=300 \mathrm{mg} / \mathrm{cm}^{2}$ and $0.007 \mathrm{~cm}=7 \mathrm{mg} / \mathrm{cm}^{2}$ when assessing dose in soft tissue.

In principle, infinitely thin phosphors made of a tissue-equivalent material, imbedded at depths of 7,300 , and $1000 \mathrm{mg} / \mathrm{cm}^{2}$ in a tissue-equivalent holder could be used to measure the skin, lens of the eye, and whole body absorbed dose directly. These phosphors, together with the TLD reader system, could be calibrated with only one type of radiation (e.g., ${ }^{137}$ Cs exposure on-phantom). When the dosimeter is irradiated by other types of radiation, such as low-energy photons or beta particles, the absorbed dose in the phosphors would change in the same manner as the absorbed dose in tissue. For radiation types having a quality factor of 1 (i.e. beta and gamma radiation), no adjustment would be required to convert absorbed dose to dose equivalent. For radiation having a quality factor greater than one (i.e. energetic neutron radiation), a knowledge of the energy spectrum at the point of interest in tissue would be required to calculate a spectrum weighted quality factor to apply to the absorbed dose to obtain dose equivalent.

(a) B.A. Rathbone, “HEDP Dose Calculation Methodology to be Implemented January 1, 2010,” September 24, 2009, letter to HEDP file. 
In actual practice however, direct measurement of absorbed dose in tissue at a specific depth, is difficult with commonly available dosimeter technology. The TLD phosphors are not infinitely thin and are not exactly tissue equivalent. The TLD cards and holders are not composed of tissue-equivalent materials. Therefore, even for radiation types having a quality factor of 1 , LiF phosphors require use of a mathematical algorithm to calculate shallow, eye, and deep absorbed doses based on the measured response of the dosimeter under a variety of exposure conditions. For neutron radiation, the energy spectrum must either be assumed (e.g. HSD) or must be inferred from element response ratios (e.g. HCND).

Algorithm development for personnel dosimeters is typically based on laboratory irradiations of dosimeters mounted on a tissue-equivalent phantom in an approximately parallel beam at normal incidence to the face of the phantom. This is the principal geometry specified in the DOELAP (DOE 1986a) and HPS (HPS 2009) performance testing standards against which dosimeter performance is evaluated. The phantom generally plays a significant role in the response of the dosimeter and is used to simulate the effect of a person's body on the dosimeter response. The backscatter factor may be defined as the ratio of tissue kerma at the surface of the phantom to that at the same point in space without the phantom present. For the standard polymethylmethacrylate (PMMA) slab phantom specified in HPS N 13.11-2009, back scatter factors range from approximately 1.1 for $662 \mathrm{keV}$ photons to approximately 1.8 for $50 \mathrm{keV}$ photons (Bartlett et al 1990, Grosswendt 1990).

Similar algorithms are used to calculate dose for environmental and nuclear accident dosimeters. Procedures used to calculate dose are described in general terms for the respective dosimeter types in Sections 5.4 through 5.10. All algorithm development by the HEDP is based on radiation exposures traceable to the NIST.

\subsubsection{Facility Calibration Codes}

When returning dosimeters for processing, contractor radiation dosimetry organizations must provide a two digit calibration code that conveys information about the radiation types and energies in the facilities where the dosimeter was worn. The information contained in this code is used by the dose algorithms when calculating dose. For each dosimeter returned for processing, this "facility calibration code" is entered into REX along with the relevant dosimeter assignment information. On a nightly basis, the combined information for each dosimeter is transmitted electronically from Hanford's Radiological Exposure (REX) system to External Dosimetry (ED) via the RETURN.TXT file. A record for each dosimeter to be processed must be included in the RETURN.TXT file.

For the HSD, the facility calibration code conveys information about whether neutron dose is expected and what the neutron energies are (i.e. what calibration factor to use for calculating neutron dose). If the calibration code under which the dosimeter is processed has been defined such that neutrons are not expected, and neutrons are detected, the algorithm will flag the calculated neutron dose result as abnormal. For Hanford, the default facility calibration code " 00 " is defined such that neutrons are expected, and when detected, a calibration factor based on bare

${ }^{252} \mathrm{Cf}$ is to be used to calculate dose. When appropriate, special facility calibration 
codes may be defined for use with the HSD, such that neutrons are not expected and/or moderated ${ }^{252} \mathrm{Cf}$ or field specific calibration factors will be used.

For the 8816 neutron TLD component of the HCND, the algorithm used to calculate neutron dose is determined from the facility calibration code. A facility calibration code $=$ " 00 " results in use of the "General Use" algorithm. For dosimeter wear periods prior to January 1 , 2010, a facility calibration code $=$ "01" results in use of a "plutonium" algorithm specific to PFP. For wear periods in 2010 and later, a facility calibration code $=$ " 01 " results in use of the "General Use" algorithm. In conjunction with implementation of the 2007 amendment to 10 CFR 835, a single "General Use" algorithm for the 8816, based on ICRP 74 fluence to dose equivalent conversion factors (ICRP 1996) was developed for use in all facilities at Hanford. However, for dosimeter wear periods in 2009, individuals who received the majority of their exposure at Hanford's Plutonium Finishing Plant (PFP) should have their dosimeters returned for processing under facility calibration code $=$ " 01 ". Regardless of monitoring year, individuals who receive a majority of their neutron dose from spent fuel, radioisotope sources (e.g. ${ }^{252} \mathrm{Cf}$, AmBe) or other sources outside PFP should have their dosimeters returned for processing under facility calibration code $=$ " 00 ". The 8816 neutron dose algorithms are described in greater detail in Section 5.5.3 and in HEDP files.

The EXT-RAD extremity "ring" dose is also based on contractor input of the facility calibration code. For rings, the two-digit calibration code provided by the contractor is divided by 10 to obtain the calibration factor. The ${ }^{137}$ Cs-based dose result is multiplied by the facility calibration factor to obtain the reported dose. Thus a code of 30 would result in the ${ }^{137} \mathrm{Cs}$-based ring result being multiplied by 3.0. When a two-digit code of " 00 " is received, the EXT-RAD algorithm interprets this as instruction to apply the default calibration factor of 2.0 established for general ring use in beta/photon fields. The factor of 2.0 compensates for potential under-response of the ring to low energy beta radiation, and potential dose gradients between maximally exposed areas of the extremity and the ring location. For plutonium work environments a calibration factor of 2.0 is also appropriate. ${ }^{\text {(a) }}$ The factor of 2 compensates for the ring's lack of response to neutrons while taking into account the ring's over response to $59 \mathrm{keV}$ photons, and is predicated on typical neutron to gamma ratios observed when working with plutonium compounds. This practice has been validated by field measurements (Scherpelz, Fix, and Rathbone 2000).

The use of facility-specific calibration factors may be necessary to compensate for technology shortfalls in dosimetry for neutron and/or low-energy beta radiation fields. Accurate assessment of dose for these radiation fields must be based on a combination of laboratory and field measurements to ensure that the dose of record is not underestimated.

(a) J. J. Fix, “Extremity Dosimetry: Neutron to Photon Ratio,” August, 1997, letter to HEDP file. 


\subsection{Hanford Standard Dosimeter}

The HSD is designed to measure shallow, eye, and deep personal dose equivalent in mixtures of beta and photon radiation fields. In addition, the dosimeter has a neutron-sensitive TLD-600 phosphor for neutron detection. Although not intended as the primary dosimeter for measuring deep personal dose equivalent from neutrons, the HSD has been DOELAP accredited in neutron exposure categories and may be used for limited monitoring of individuals who are not likely to receive more than 100 mrem of neutron dose per year. The HSD is calibrated to measure and report deep personal dose equivalent from neutrons based on the ICRP 60 Q-LET relationship (ICRP 1991), consistent with the 2007 amendment to 10 CFR 835 (DOE 2007).

\subsubsection{General Features}

The HSD holder was designed according to HEDP specifications and is commercially available as a Harshaw 8825. The dosimeter card contains TLD700 phosphors in positions one, two, and three and a TLD-600 phosphor in position four. These phosphors have thicknesses of $0.38 \mathrm{~mm}\left(100 \mathrm{mg} / \mathrm{cm}^{2}\right)$ in positions one, two, and four and $0.15 \mathrm{~mm}\left(40 \mathrm{mg} / \mathrm{cm}^{2}\right)$ in position three. The holder filtration consists of $242 \mathrm{mg} / \mathrm{cm}^{2}$ ABS plastic plus $91 \mathrm{mg} / \mathrm{cm}^{2}$ copper over position one, $1000 \mathrm{mg} / \mathrm{cm}^{2}$ ABS and polytetrafluorethylene (PTFE) plastic over position two, $8 \mathrm{mg} / \mathrm{cm}^{2}$ Teflon ${ }^{\circledR}$ and $9 \mathrm{mg} / \mathrm{cm}^{2}$ Mylar ${ }^{\circledR}$ over position three, and $240 \mathrm{mg} / \mathrm{cm}^{2}$ ABS plastic plus $463 \mathrm{mg} / \mathrm{cm}^{2}$ tin over position four.

This dosimeter is illustrated in Figure 5.9. The dosimeter holder is constructed of black ABS plastic. The filter type and thickness is identified for each position of the dosimeter holder for the front side (i.e., dosimeter side facing away from the body). There are no filters on the back side. The density thickness of the ABS plastic case on the backside is $173 \mathrm{mg} / \mathrm{cm}^{2}$. A red-tinted viewing window is centered on the back side of the dosimeter holder. The viewing window is used to electronically read the permanent ID number of the card enclosed within the holder.

\subsubsection{Dosimeter Assignment and Processing Protocol}

The HSD cards and holders must routinely satisfy a number of QC checks to be eligible for issuance to Hanford contractor dosimetry organizations and, upon return, to be eligible for routine processing. Assuming that a dosimeter holder and card have been qualified for use within the HEDP, the following series of actions, tracked by computer, must be taken to issue them:

- Dosimeter cards are processed through one of the automated 8800 reader systems to conduct a pre-issue reader "annealing." This reader processing cycle ensures that any remaining residual signal from past occupational use of the card or environmental background radiation is removed. Processing results for each card must satisfy established tolerance limits as one step in the overall qualification of a card for assignment. 


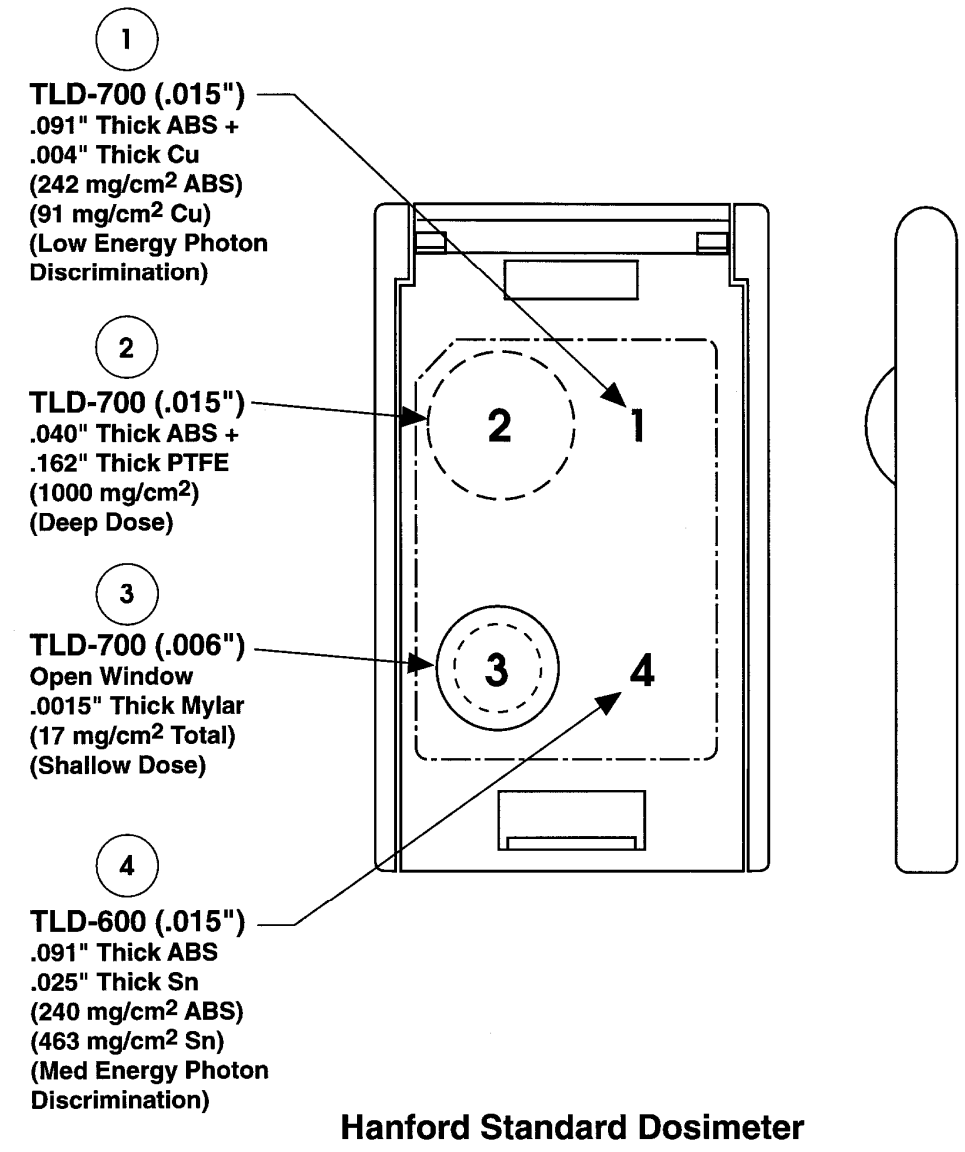

39502026.3

Figure 5.9 Hanford Standard Dosimeter

- All dosimeter cards are oven-annealed at $80^{\circ} \mathrm{C}$ for 16 hours before being loaded into a holder. This low temperature annealing process reduces the significance of signal fading by reducing the number of traps contributing to the lower-temperature peaks. It does not clear the higher temperature dosimetric traps to any significant extent. Use of this oven anneal reduces long-term fade to less than $15 \%$ per year. ${ }^{\text {(a) }}$ Studies of various anneal treatment at Los Alamos National Laboratory have shown that this preirradiation oven-annealing technique provides improved reproducibility over other methods studied (Storm et al. 1981; Cortez, Storm, and Littlejohn, 1977).

- Card and holder assignments are electronically recorded when a dosimeter is issued.

(a) W. V. Baumgartner, "Study of Environmental Buildup and Fade for 8825 Card," October 11, 1994, letter to HEDP file. 
- Upon return for processing, card and holder pairing is electronically checked to see if the dosimeter (i.e., card and holder) is being returned identically as issued.

- Cards are processed for a total read-out time of 13.3 seconds using the reader TTP parameters in Section 5.2.5.

- Throughout the processing, there are numerous reader parameters that must be satisfied for processing to continue. Selected portions of the glow curve, encompassing the dosimetric peaks, are used for dose calculation, thereby improving the signal-to-noise characteristics.

- Glow curves are electronically recorded for all personnel dosimeter processing. Plots of the TTP and the glow curve data visually demonstrate the rate at which light is being received by the PMT during processing of the dosimeter cards. These data are analyzed on the External Dosimetry Data Management System to validate the quality of the processing data.

\subsubsection{Algorithm}

The HSD measures the personal dose equivalent at depths of 7, 300, and $1000 \mathrm{mg} / \mathrm{cm}^{2}$ from beta and photon radiation. Primary calibration of the HSD algorithm is based on dosimeter response to ${ }^{137} \mathrm{Cs}$ when irradiated on a $30 \mathrm{x} 30 \mathrm{x}$ $15 \mathrm{~cm}^{3}$ (PMMA) phantom in the geometry specified in the Department of Energy Standard for the Performance Testing of Personnel Dosimetry Systems DOE/EH0027 (DOE 1986a). Algorithm response functions used for calculation of shallow and deep personal dose equivalent quantities from adjusted element readings were developed from the adjusted element responses to each source and source mixture specified in the standard and the delivered shallow and deep personal dose equivalents for those irradiations. The eye dose functions for photons were developed from irradiations to the NIST filtered x-ray sources specified in DOE/EH-0027 and HPS N 13.11-1993 (HPS 1993) and the NIST $C_{k}$ factors for those sources. The eye dose function for beta radiation was developed using beta sources specified in the standards plus additional PNNL beta sources, and the measured dose rates at a depth of $300 \mathrm{mg} / \mathrm{cm}^{2}$ for those sources.

The functions used to calculate shallow and deep personal dose equivalent when the radiation type is primarily low energy photons or mixtures of photons and beta particles were developed by Bicron/NE using a neural network (Moscovitch and Rotunda 1996). The application of neural networks in developing dose algorithms for multi-element dosimeters is described by Marko Moscovitch (Moscovitch 1999) and in Bicron/NE 8825 algorithm documentation (Bicron/NE 1999).

The algorithm has two major steps that must be completed in sequence to determine the personal dose equivalents $H_{p}(0.07), H_{p}(3), H_{p}(10)_{\gamma}$ and $H_{p}(10)_{\eta}$. First, the algorithm identifies the type(s) of radiation to which a dosimeter was exposed by comparing adjusted element ratios with those established for known radiation types. The algorithm then uses dose response functions for the shallow, eye and deep dose elements established for the identified radiation type, to calculate personal dose equivalent at the specified tissue depths. For mixtures of beta and photon radiation, the algorithm first determines the total shallow and eye 
personal dose equivalent and then estimates the proportion of each due to beta or photon radiation. For deep dose from beta-photon mixtures, the entire deep dose equivalent is assumed to be from photon radiation. For deep dose from neutronphoton mixtures, the neutron component and photon component are calculated independently. The functions used for calculating dose from mixtures of radiation are more complicated than for single sources.

Although the HSD algorithm employs ratio tests to determine the radiation type and contains functions used to calculate the appropriate calibration factors to be applied to adjusted chip readings to obtain dose, there are some circumstances where it is desirable or necessary for the user to specify (either directly or indirectly) the radiation types and/or calibration factors to be used. Typically, these are situations where neutrons are present and the TLD-600 chip behind tin filtration cannot be used for photon energy discrimination. There may also be situations where field conditions are very well known and the most accurate results can be obtained by the application of site-specific calibration factors.

\subsubsection{HSD Element Response to DOELAP Sources}

The relative element response of individual chips in the HSD to known amounts of shallow and deep personal dose equivalent from a variety of radiation sources is shown in Table 5.6. The values in this table were obtained from HSD irradiations to sources, geometry, and beam quality specifications contained in the DOELAP standard (DOE 1986a). The deep dose response values for bare and moderated ${ }^{252} \mathrm{Cf}$ (shaded) have been updated to reflect a re-evaluation of the $\mathrm{H}_{\mathrm{p}}(10)_{\eta}$ delivered from the PNNL sources that were used to develop these data. The updated neutron response values in Table 5.6 reflect a $49 \%$ increase in assessed $\mathrm{H}_{\mathrm{p}}(10)_{\eta}$ for the $\mathrm{D}_{2} \mathrm{O}$ moderated ${ }^{252} \mathrm{Cf}$ source and a $29 \%$ increase for the bare ${ }^{252} \mathrm{Cf}$ source. The new values for $\mathrm{H}_{\mathrm{p}}(10)_{\eta}$ dose rate are based on ICRP 74 fluence to dose equivalent conversion factors and recent assessments of the neutron energy spectrum from modeling and measurement.

The calibrated element response is the response of a chip when read with ECC and RCF applied. Five dosimeters were irradiated on-phantom to approximately 500 mrem (shallow dose) for each radiation type. The calibrated element response $(\mathrm{mR})$ of each chip was divided by the applied shallow or deep personal dose equivalent (mrem) to obtain response factors $(\mathrm{mR} / \mathrm{mrem})$. For each radiation type, the mean response factor for each chip position was normalized to the response factor for ${ }^{137}$ Cs to obtain the tabled relative values.

\subsubsection{Algorithm Bias}

In general, the response of the HSD algorithm has been well documented in formal DOELAP and NVLAP performance test reports, as well as in studies documented in HEDP files. ${ }^{(a)}$ [HEDP no longer maintains NVLAP accreditation.] In preparation for initial DOELAP performance testing, groups of five HSDs each were irradiated to the sources in Table 5.7 according to the specifications given in the DOELAP standard (DOE 1986a). The mean reported

(a) B. A. Rathbone, “HSD Performance Testing,” January 25, 1996, letter to HEDP file.

B. A. Rathbone, "HSD Performance Testing Using NVLAP Criteria and Ck”, September 30, 1997, letter to HEDP file. 
deep personal dose equivalent calculated by the algorithm was within $\pm 15 \%$ of the given dose for each source used. The $\mathrm{H}_{\mathrm{p}}(07)$ and $\mathrm{H}_{\mathrm{p}}(10)_{\gamma}$ response of the algorithm for the various sources tested is shown in Table 5.7.

In conjunction with verification of the algorithm response for $\mathrm{H}_{\mathrm{p}}(07)$ and $\mathrm{H}_{\mathrm{p}}(10)_{\gamma}$, the HSD algorithm was also verified for $\mathrm{H}_{\mathrm{p}}(3)$ response. The delivered $\mathrm{H}_{\mathrm{p}}(3)$ for various NIST filtered X-ray techniques and for ${ }^{137} \mathrm{Cs}$ was based on $\mathrm{C}_{\mathrm{k}}$ factors published by NIST. ${ }^{\text {(a) }}$ The delivered $\mathrm{H}_{\mathrm{p}}$ (3) for the K16 technique is based on $\mathrm{C}_{\mathrm{k}}$ factors for monoenergetic photons and slab phantom (Grosswendt 1990). The algorithm's $H_{p}(3)$ response for various sources is shown in Table 5.8. A complete evaluation of HSD bias and precision relative to delivered shallow, eye, and deep personal dose equivalent, from both pure sources and mixtures is documented in HEDP files. ${ }^{\text {(b) }}$

Table 5.6. HSD Element Response to DOELAP Sources

\begin{tabular}{|c|c|c|c|c|c|c|c|c|}
\hline \multirow{3}{*}{$\begin{array}{l}\text { Beam } \\
\text { Code }\end{array}$} & \multicolumn{8}{|c|}{ Element Response Per Unit Delivered Personal Dose Equivalent (normalized to ${ }^{137}$ Cs response) } \\
\hline & \multicolumn{4}{|c|}{ Shallow Dose Response ${ }^{a}$} & \multicolumn{4}{|c|}{ Deep Dose Response ${ }^{b}$} \\
\hline & chip1 & chip2 & chip3 & chip4 & chip1 & chip2 & chip3 & chip4 \\
\hline M30 & 0.13 & 0.41 & 1.25 & 0.06 & 0.32 & 0.99 & 2.99 & 0.14 \\
\hline S60 & 1.09 & 1.32 & 1.57 & 0.48 & 1.17 & 1.41 & 1.69 & 0.52 \\
\hline M150 & 1.35 & 1.29 & 1.32 & 0.86 & 1.30 & 1.24 & 1.26 & 0.82 \\
\hline K16 & 0.07 & 0.46 & 1.32 & 0.04 & 0.21 & 1.32 & 3.74 & 0.13 \\
\hline K59 & 1.39 & 1.31 & 1.35 & 0.79 & 1.33 & 1.25 & 1.29 & 0.75 \\
\hline H150 & 1.15 & 1.06 & 1.12 & 0.91 & 1.15 & 1.06 & 1.12 & 0.91 \\
\hline${ }^{90} \mathrm{Sr} /{ }^{90} \mathrm{Y}$ & 0.22 & 0.02 & 1.09 & 0.01 & N/A & N/A & N/A & N/A \\
\hline${ }^{204} \mathrm{Tl}$ & 0.00 & 0.00 & 0.65 & 0.00 & N/A & N/A & N/A & N/A \\
\hline DU & 0.09 & 0.02 & 0.57 & 0.02 & N/A & N/A & N/A & N/A \\
\hline${ }^{137} \mathrm{Cs}$ & 1.00 & 1.00 & 1.00 & 1.00 & 1.00 & 1.00 & 1.00 & 1.00 \\
\hline${ }^{241} \mathrm{Am}$ & 1.39 & 1.30 & 1.33 & 0.79 & 1.33 & 1.24 & 1.28 & 0.75 \\
\hline${ }^{252} \mathrm{Cf} \mathrm{M}$ & $1.18^{\mathrm{C}}$ & $1.07^{\mathrm{C}}$ & $1.16^{\mathrm{c}}$ & $57.03^{c}$ & $0.12^{\mathrm{d}}$ & $0.11^{d}$ & $0.12^{\mathrm{d}}$ & $5.72^{\mathrm{d}}$ \\
\hline${ }^{252} \mathrm{Cf} \mathrm{U}$ & $0.90^{\mathrm{C}}$ & $0.83^{\mathrm{C}}$ & $0.99^{c}$ & $18.47^{c}$ & $0.05^{\mathrm{d}}$ & $0.04^{d}$ & $0.05^{\mathrm{d}}$ & $0.94^{\mathrm{d}}$ \\
\hline
\end{tabular}

a. Response of chips per unit of delivered shallow personal dose equivalent, normalized to the ${ }^{137}$ Cs response.

b. Response of chips per unit of delivered deep personal dose equivalent, normalized to the ${ }^{137} \mathrm{Cs}$ response.

c. Delivered shallow personal dose equivalent from ${ }^{252} \mathrm{Cf}$ photons only was used to calculate these values.

d. Delivered deep personal dose equivalent from photons and neutrons was used to calculate these values.

(a) C. G. Soares and P. R. Martin, “A comprehensive Set of Conversion Coefficients for Photons,” Proceedings of Bicron/NE TLD User’s Symposium held in Las Vegas, NV; March 13-17, 1995.

(b) B. A. Rathbone, “HSD Performance Testing Using NVLAP Criteria and Ck,” September 30, 1997, HEDP file. 
Table 5.7. HSD Algorithm Shallow and Deep Dose Response

\begin{tabular}{|c|c|c|c|}
\hline Beam Code & $\begin{array}{c}\text { Average Energy } \\
\text { (keV) }\end{array}$ & $\begin{array}{c}\text { Shallow Dose } \\
\text { Response }^{\mathrm{a}}\end{array}$ & $\begin{array}{l}\text { Deep Dose } \\
\text { Response }^{d}\end{array}$ \\
\hline K16 & 16 & 1.03 & 1.09 \\
\hline M30 & 20 & 1.01 & 0.94 \\
\hline S60 & 36 & 1.11 & 1.03 \\
\hline K59 & 59 & 0.92 & 0.91 \\
\hline${ }^{241} \mathrm{Am}$ & 59 & 0.92 & 0.91 \\
\hline M150 & 70 & 0.94 & 0.93 \\
\hline H150 & 117 & 0.95 & 0.93 \\
\hline${ }^{13 /} \mathrm{Cs}$ & 662 & 1.00 & 1.00 \\
\hline${ }^{90} \mathrm{Sr} /{ }^{90} \mathrm{Y}^{\mathrm{b}}$ & $931^{\mathrm{C}}$ & 1.04 & $\mathrm{n} / \mathrm{a}$ \\
\hline${ }^{204} \mathrm{Tl}^{\mathrm{D}}$ & $267^{c}$ & 0.95 & $\mathrm{n} / \mathrm{a}$ \\
\hline
\end{tabular}

a. Reported/given dose equivalent. Normalized to ${ }^{137} \mathrm{Cs}$ value.

b. Source specifications described in DOELAP standard (DOE 1986a).

c. Nominal values. Actual energies are slightly less because of filtration inherent in encapsulation and beam flattener.

$\mathrm{n} / \mathrm{a}=$ Not applicable.

Table 5.8 HSD Algorithm Eye Dose Response

\begin{tabular}{||c|c|c||}
\hline Beam Code & $\begin{array}{c}\text { Average Energy } \\
(\mathrm{keV})\end{array}$ & Reported/Given $^{\mathrm{a}}$ \\
\hline K16 & 16 & 0.61 \\
\hline M30 & 20 & 1.06 \\
\hline M60 & 34 & 1.01 \\
\hline S60 & 38 & 1.11 \\
\hline M100 & 51 & 1.11 \\
\hline${ }^{241} \mathrm{Am}$ & 59 & 0.96 \\
\hline $\mathrm{M} 150$ & 70 & 0.95 \\
\hline $\mathrm{H} 150$ & 117 & 0.95 \\
\hline${ }^{137} \mathrm{Cs}$ & 662 & 1.00 \\
\hline${ }^{90} \mathrm{Sr} /{ }^{90} \mathrm{Y}^{\mathrm{b}}$ & $931^{\mathrm{c}}$ & 1.02 \\
\hline
\end{tabular}

a. Reported value normalized to reported ${ }^{137}$ Cs value

b. Source specifications described in DOELAP standard (DOE 1986a)

c. Nominal values. Actual energies are slightly less because of filtration inherent in encapsulation and beam flattener 
Table 5.9 HSD Algorithm Neutron Deep Dose Response

\begin{tabular}{||l|c|c|l||}
\hline \hline Source or Field & $\begin{array}{c}\text { Average Energy } \\
(\mathrm{keV})\end{array}$ & $\begin{array}{c}\text { Reported } \\
\text { Given }^{\text {a }}\end{array}$ & $\begin{array}{l}\text { Exposure } \\
\text { Geometry b,c, }\end{array}$ \\
\hline AmBe (bare) & 4160 & 0.7 & AP @ 50 cm \\
\hline${ }^{252} \mathrm{Cf}$ (bare) & 2130 & 1.0 & AP @ $50 \mathrm{~cm}$ \\
\hline${ }^{252} \mathrm{Cf}\left(\mathrm{D}_{2} \mathrm{O}\right.$ moderated with Cd) & 550 & 8.2 & AP @ $50 \mathrm{~cm}$ \\
\hline${ }^{252} \mathrm{Cf}\left(\mathrm{D}_{2} \mathrm{O}\right.$ moderated w/o/Cd) & 550 & 11 & AP @ $50 \mathrm{~cm}$ \\
\hline PWR fuel in NAC-1 shipping cask & $\mathrm{n} / \mathrm{a}$ & $4.3^{\mathrm{e}}$ & AP @ $1.7 \mathrm{~m}$ \\
\hline PWR fuel in NAC-1 shipping cask & $\mathrm{n} / \mathrm{a}$ & $6.2^{\mathrm{e}}$ & AP @ $11.2 \mathrm{~m}$ \\
\hline PWR fuel in NAC-1 shipping cask & $150-200^{\mathrm{f}}$ & $4.8^{\mathrm{g}}$ & ROT @ $2.2 \mathrm{~m}$ \\
\hline $\begin{array}{l}\text { PWR fuel in NAC-1 shipping cask } \\
\text { behind 2 ft. thick concrete shield wall }\end{array}$ & $70^{\mathrm{f}}$ & $11^{\mathrm{g}}$ & ROT @ $20.5 \mathrm{~m}$ \\
\hline PFP “front side” & $120-360^{\mathrm{h}}$ & $6^{\mathrm{h}}$ & ISO, ROT \\
\hline PFP “back side” & $150-740^{\mathrm{h}, \mathrm{i}}$ & $2-18^{\mathrm{j}}$ & ISO, ROT \\
\hline \hline
\end{tabular}

a. Given neutron dose equivalent based on TEPC measurement. R/G standard uncertainty $=10 \%$

b. $\mathrm{AP}=$ anterior-posterior orientation (facing source)

c. $\mathrm{ISO}=$ isotropic radiation

d. ROT = rotational orientation with respect to source

e. B. A. Rathbone, "Neutron Response of HSD and HCND Personnel Dosimeters Near Spent Fuel Casks", February 13, 2004, HEDP file

f. R. I. Scherpelz, "Neutron Measurements on the ISA Pad", December 9, 2003, HEDP File

g. B. A. Rathbone, "Neutron Dose Algorithm Verification at ISA", September 25, 2006, HEDP file

h. B. A. Rathbone, "Neutron Calibration Factors for HSD in Plutonium Environments", August 20, 1997, HEDP file

i. R. I. Scherpelz and B. A. Rathbone, "Neutron Measurements at PFP August-September,2003”, November 14, 2003, HEDP file

j. B. A. Rathbone, "Verification of 8816 Performance in PFP Neutron Fields", March 3, 2004, HEDP file

The HSD does not have a true (energy compensating) neutron dose algorithm. Instead, it calculates neutron dose using a neutron calibration factor that is assigned based on the facility calibration code. When the dosimeter detects neutron dose and has been processed under facility calibration code $=00$ (Hanford default), the neutron dose calculation will be based on a calibration of the dosimeter to an unmoderated ${ }^{252} \mathrm{Cf}$ neutron energy spectrum. Most neutron dose detected by the HSD will likely be from scattered neutrons originating from distant or shielded sources. When this is the case, the HSD will over respond due to the moderation involved. An important exception where the HSD has the potential to under respond is exposure to an unshielded AmBe source (e.g. well logging source).

The HSD's neutron response (relative to TEPC) to various laboratory and field sources when processed using the default calibration code is shown in Table 5.9. These measurements were made under the "old” system of ICRP 21 neutron quality factors and Q-LET relationship, and are provided here for information purposes only. Although these data are based on the "old" system, the R/G ratios are likely within $\pm 25 \%$ of the values that would be obtained under the current system. The basis for this assumption is as follows: A comparison of ICRP 21 based fluence to dose equivalent conversion factors from ISO 8925-1989 (ISO 1989) and ICRP 74 based fluence to dose equivalent conversion factors from ISO 8529:3-1998 (ISO 1998) shows fairly modest and uniform increases for 
moderated ${ }^{252} \mathrm{Cf}$, bare ${ }^{252} \mathrm{Cf}$, and bare ${ }^{241} \mathrm{AmBe}$ neutron energy spectra. The ratios of new/old conversion factors are: $\mathrm{D}_{2} \mathrm{O}$ moderated ${ }^{252} \mathrm{Cf}(1.21)$, bare ${ }^{252} \mathrm{Cf}(1.18)$, bare ${ }^{241} \mathrm{AmBe}(1.08)$. This represents a $12 \%$ range of increases. For an HSD calibrated to bare californium, the "reported" $(\mathrm{R})$ values for this dosimeter under the new system would be expected to be 1.18 times the reported values under the old system, regardless of field spectra. For energy independent field measurement instruments (e.g. TEPC or multisphere), the measured dose for an ${ }^{241} \mathrm{AmBe}$ neutron field under the new system would be 1.08 times the measured dose under the old system. Thus the reference or "given" $(G)$ value for ${ }^{241} \mathrm{AmBe}$ would increase by a factor of 1.08 . The combined effect of the increase in reported (R) value and the increase in "given" $(\mathrm{G})$ value would be that for ${ }^{241} \mathrm{AmBe}$ neutron fields, the R/G value for the HSD under the new system of measurement would be 1.09 times the R/G value observed under the old system. Similarly, for $\mathrm{D}_{2} \mathrm{O}$ moderated ${ }^{252} \mathrm{Cf}$ neutron fields, the R/G value for the HSD under the new system of measurement would be 0.98 times the $R / G$ value observed under the old system. To the extent that the range of neutron energies in Table $\mathbf{5 . 9}$ are larger than those represented in the ISO 8529 standards, larger changes in R/G might be expected for Table 5.9. However, it is unlikely that the changes would exceed 25\% in either direction. A repeat of the measurements in Table $\mathbf{5 . 9}$ with both the field instrument and the dosimeter calibrated under the new system would be expected to produce, $\mathrm{R} / \mathrm{G}$ values similar to those shown in Table 5.9. Given the uncertainty of the original measurements, and neutron dosimetry in general, the values in Table 5.9 should still be considered useful indicators of the extent to which HSDs processed with the default bare ${ }^{252} \mathrm{Cf}$ calibration factor will over respond under most field conditions at Hanford.

\subsubsection{Angular Response}

Experimental Method

The angular response of the HSD was measured as required by DOELAP (DOE 1986a), which requires documentation of the angular response for each dosimeter design in irradiation categories III through VI. ${ }^{\text {(a) }}$ The HPS had also identified angular response testing at $\pm 40^{\circ}$ (HPS 1993) in addition to the $\pm 30^{\circ}, \pm 60^{\circ}$, and $\pm 85^{\circ}$ angles already identified in the DOELAP standard so this angle was included as well.

Measurement of angular response was conducted using irradiations from selected beta, photon, and neutron sources. Irradiation geometries are summarized as follows:

- Photon irradiations were performed using a phantom measuring $30 \mathrm{~cm}$ x 30 $\mathrm{cm} \times 15 \mathrm{~cm}$. Irradiations were performed using k-fluorescent K16, M30, $\mathrm{S} 60,{ }^{241} \mathrm{Am}$, and ${ }^{137} \mathrm{Cs}$ sources. Irradiation distances from the source center to the front edge vertical centerline of the phantom were $50 \mathrm{~cm}$ for $\mathrm{K}_{16}{ }^{(\mathrm{b})}$ and ${ }^{241} \mathrm{Am}$ irradiations and $100 \mathrm{~cm}$ for ${ }^{137} \mathrm{Cs}$ irradiations.

(a) J. J. Fix,“Angular Response of Hanford Personnel Dosimeters,” October 18, 1994, HEDP file. B. A. Rathbone, “Angular Dependence Study for Hanford Standard Dosimeter," December 17, 1997, HEDP file. J. J. Fix, “HSD Neutron Angular Response,” May 15, 1998, HEDP file.

(b) Surface of target to surface of phantom. 
- Beta exposures were made in a similar manner using a $30 \mathrm{~cm}$ x $30 \mathrm{~cm}$ x $5 \mathrm{~cm}$ phantom. $\quad \mathrm{A}^{90} \mathrm{Sr} /{ }^{90} \mathrm{Y}$ source and a ${ }^{204} \mathrm{Tl}$ source were used to irradiate dosimeters on the phantom located a distance of $35 \mathrm{~cm}$ from the source.

- Neutron measurements were made with bare and moderated ${ }^{252} \mathrm{Cf}$ sources. A phantom measuring $40 \mathrm{~cm} \times 40 \mathrm{~cm} \times 15 \mathrm{~cm}$ was used at a distance of $50 \mathrm{~cm}$ from the source.

All source calibrations are traceable to NIST. Irradiations were timed to deliver an approximate dose of $5 \mathrm{mSv}$ (500 mrem), in reference to the $0^{\circ}$ angle (normal) exposure geometry, to each dosimeter.

To measure angular response, the phantom was rotated to each of the angles of $0^{\circ}, 30^{\circ}, 40^{\circ}, 60^{\circ}$, and $85^{\circ}$ clockwise and counter-clockwise as viewed from overhead. Dosimeters were mounted on thin Plexiglas ${ }^{\mathrm{TM}}$ sheets that were then mounted on the front of the phantom. Dosimeters were mounted upright with the beta window at the "top" to measure the vertical angular response and horizontally (i.e., dosimeter rotated $90^{\circ}$ clockwise so that long axis is horizontal) to measure the horizontal angular response. Figure $\mathbf{5 . 1 0}$ illustrates the exposure setup. Two of the three dosimeters exposed at each angle were mounted on the phantom surface for one exposure while the third dosimeter was mounted in the center (similar to the HCND) and exposed separately. For all irradiations, a distance of at least $7.5 \mathrm{~cm}$ was maintained between the dosimeters and the edge of the $30 \mathrm{~cm} \times 30 \mathrm{~cm} \times 15 \mathrm{~cm}$ phantom for beta and photon irradiations, and a distance of at least $10.0 \mathrm{~cm}$ was maintained between the dosimeters and the edge of the $40 \mathrm{~cm} \times 40 \mathrm{~cm} \times 15 \mathrm{~cm}$ phantom for neutron irradiations, in accordance with HPS N13.11-1993 (HPS 1993) and DOELAP (DOE 1986a) requirements,.

\section{Vertical Rotation}

Midline of Front Face

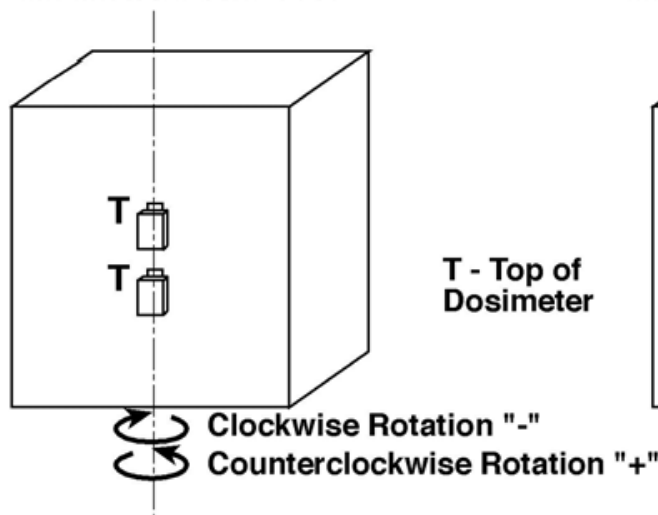

\section{Horizontal Rotation}

Midline of Front Face

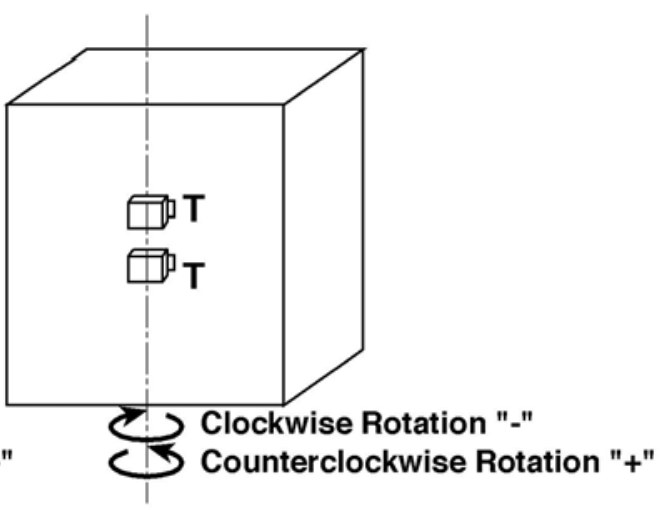

39502026.1

Figure 5.10. Irradiation Setup for Dosimeter Angular Response Evaluation

Results

The results of the study are shown in Tables 5.10 and 5.11, and shown graphically in Figures 5.11 - $\mathbf{5 . 1 6}$ for several sources of irradiation. These figures illustrate the ratio of the reported dose for each angle normalized to the dosimeter response at $0^{\circ}$ for horizontal and vertical dosimeter rotation, respectively. In these plots, the angles for the clockwise rotation are considered 
to be negative; counter-clockwise rotations are positive. Although the angular response data for bare and moderated ${ }^{252} \mathrm{Cf}$ were determined from delivered doses based on ICRP 21, the angular response data are still valid for neutron doses based on ICRP 74. The energy spectra have not changed, and the light output per unit fluence striking the dosimeter for any given spectrum has not changed. Only the calibration factor used in the algorithm has changed and the factor is a constant, (i.e. does not vary as a function of angle). Because the responses at angles are normalized to the response at zero degrees, their values have not changed.

Table 5.10. HSD Shallow Dose Angular Response

\begin{tabular}{||c|c|c|c|c|c|c|c|c|c|c|c||}
\hline \hline Source & $\begin{array}{c}\text { Average } \\
\text { Energy } \\
(\mathrm{keV})\end{array}$ & $\begin{array}{c}\text { Axis of } \\
\text { Rotation }\end{array}$ & $-85^{\circ}$ & $-60^{\circ}$ & $-40^{\circ}$ & $-30^{\circ}$ & $0^{\circ}$ & $30^{\circ}$ & $40^{\circ}$ & $60^{\circ}$ & $85^{\circ}$ \\
\hline $\mathrm{K} 16$ & 16 & $\mathrm{H}$ & 0.02 & 0.93 & 1.18 & 1.23 & 1.00 & 1.00 & 1.08 & 1.08 & 0.59 \\
\hline & & $\mathrm{V}$ & 0.04 & 1.00 & 1.24 & 1.23 & 1.00 & 1.28 & 1.27 & 1.04 & 0.10 \\
\hline $\mathrm{M} 30$ & 20 & $\mathrm{H}$ & 0.07 & 0.94 & 1.07 & 1.16 & 1.00 & 0.88 & 0.76 & 0.56 & 0.10 \\
\hline & & $\mathrm{V}$ & 0.12 & 0.83 & 0.89 & 0.95 & 1.00 & 0.92 & 0.96 & 0.64 & 0.04 \\
\hline $\mathrm{S} 60$ & 36 & $\mathrm{H}$ & 0.42 & 1.02 & 1.05 & 1.06 & 1.00 & 0.92 & 0.92 & 0.71 & 0.59 \\
\hline & & $\mathrm{V}$ & 0.47 & 0.89 & 1.02 & 0.99 & 1.00 & 0.97 & 1.01 & 0.87 & 0.20 \\
\hline${ }^{241} \mathrm{Am}$ & 59 & $\mathrm{H}$ & 0.58 & 0.80 & 0.89 & 0.96 & 1.00 & 1.11 & 1.20 & 1.20 & 0.66 \\
\hline & & $\mathrm{V}$ & 0.35 & 0.87 & 0.94 & 0.95 & 1.00 & 0.97 & 0.94 & 0.90 & 0.79 \\
\hline${ }^{137} \mathrm{Cs}$ & 662 & $\mathrm{H}$ & 0.72 & 0.95 & 0.96 & 1.00 & 1.00 & 1.05 & 1.06 & 1.08 & 0.66 \\
\hline & & $\mathrm{V}$ & 0.71 & 1.03 & 1.02 & 1.03 & 1.00 & 1.00 & 1.01 & 0.97 & 0.91 \\
\hline${ }^{90} \mathrm{Sr} /{ }^{90} \mathrm{Y}$ & 931 & $\mathrm{H}$ & 0.04 & 0.24 & 0.85 & 0.92 & 1.00 & 1.27 & 1.48 & 0.75 & 0.06 \\
\hline & & $\mathrm{V}$ & 0.05 & 0.36 & 0.94 & 1.02 & 1.00 & 1.17 & 1.12 & 0.83 & 0.05 \\
\hline${ }^{204} \mathrm{Tl}$ & 267 & $\mathrm{H}$ & 0.08 & 0.39 & 0.74 & 0.92 & 1.00 & 0.78 & 0.55 & 0.26 & 0.05 \\
\hline & & $\mathrm{V}$ & 0.07 & 0.33 & 0.66 & 0.86 & 1.00 & 0.81 & 0.57 & 0.31 & 0.07 \\
\hline
\end{tabular}

Table 5.11. HSD Deep Dose ${ }^{(a)}$ Angular Response

\begin{tabular}{||c|c|c|c|c|c|c|c|c|c|c|c||}
\hline \hline Source & $\begin{array}{c}\text { Average } \\
\text { Energy } \\
\text { (keV) }\end{array}$ & $\begin{array}{c}\text { Axis of } \\
\text { Rotation }\end{array}$ & $-85^{\circ}$ & $-60^{\circ}$ & $-40^{\circ}$ & $-30^{\circ}$ & $0^{\circ}$ & $30^{\circ}$ & $40^{\circ}$ & $60^{\circ}$ & $85^{\circ}$ \\
\hline K16 & 16 & $\mathrm{H}$ & 0.35 & 1.89 & 1.77 & 1.63 & 1.00 & 1.41 & 1.43 & 1.45 & 0.11 \\
\hline & & $\mathrm{V}$ & 0.19 & 1.59 & 1.63 & 1.64 & 1.00 & 1.51 & 1.61 & 1.75 & 0.33 \\
\hline $\mathrm{M} 30$ & 20 & $\mathrm{H}$ & 0.09 & 1.20 & 1.00 & 1.01 & 1.00 & 0.98 & 0.93 & 0.92 & 0.30 \\
\hline & & $\mathrm{V}$ & 0.38 & 1.30 & 0.98 & 1.01 & 1.00 & 1.04 & 1.04 & 1.06 & 0.13 \\
\hline $\mathrm{S} 60$ & 36 & $\mathrm{H}$ & 0.33 & 0.89 & 0.95 & 0.99 & 1.00 & 1.00 & 1.00 & 0.92 & 0.80 \\
\hline & & $\mathrm{V}$ & 0.63 & 0.94 & 0.96 & 1.02 & 1.00 & 0.98 & 0.94 & 0.88 & 0.27 \\
\hline${ }^{241} \mathrm{Am}$ & 59 & $\mathrm{H}$ & 0.97 & 0.93 & 0.96 & 1.01 & 1.00 & 1.00 & 1.02 & 0.94 & 0.43 \\
\hline & & $\mathrm{V}$ & 0.59 & 0.89 & 0.95 & 0.96 & 1.00 & 0.97 & 0.94 & 0.92 & 0.75 \\
\hline${ }^{137} \mathrm{Cs}$ & 662 & $\mathrm{H}$ & 1.00 & 1.03 & 1.01 & 1.02 & 1.00 & 1.02 & 1.05 & 1.07 & 0.61 \\
\hline & & $\mathrm{V}$ & 0.91 & 1.06 & 1.04 & 1.03 & 1.00 & 1.00 & 1.03 & 1.02 & 0.98 \\
\hline${ }^{252} \mathrm{Cf} \mathrm{U}$ & 2100 & $\mathrm{H}$ & 0.22 & 0.63 & 0.86 & 0.90 & 1.00 & 0.96 & 0.92 & 0.72 & 0.33 \\
\hline & & $\mathrm{V}$ & 0.28 & 0.70 & 0.93 & 0.91 & 1.00 & 0.90 & 0.86 & 0.65 & 0.26 \\
\hline${ }^{252} \mathrm{Cf} \mathrm{M}$ & 550 & $\mathrm{H}$ & 0.16 & 0.53 & 0.78 & 0.84 & 1.00 & 0.96 & 0.90 & 0.65 & 0.22 \\
\hline & & $\mathrm{V}$ & 0.23 & 0.59 & 0.84 & 0.92 & 1.00 & 0.88 & 0.80 & 0.58 & 0.19 \\
\hline
\end{tabular}

(a) For Cf-252 irradiations, neutron component only. 

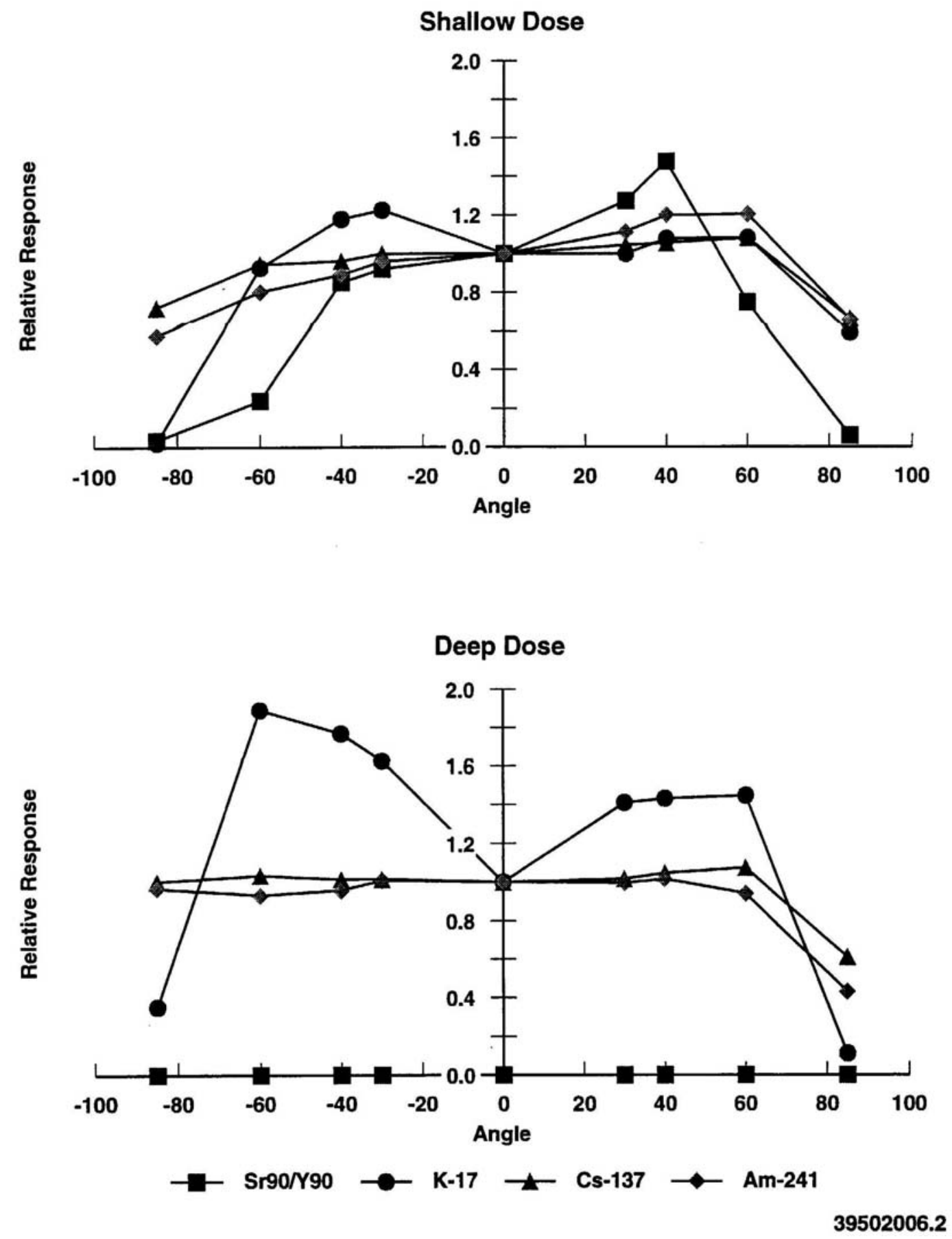

Figure 5.11. HSD Angular Response - Horizontal Rotation 

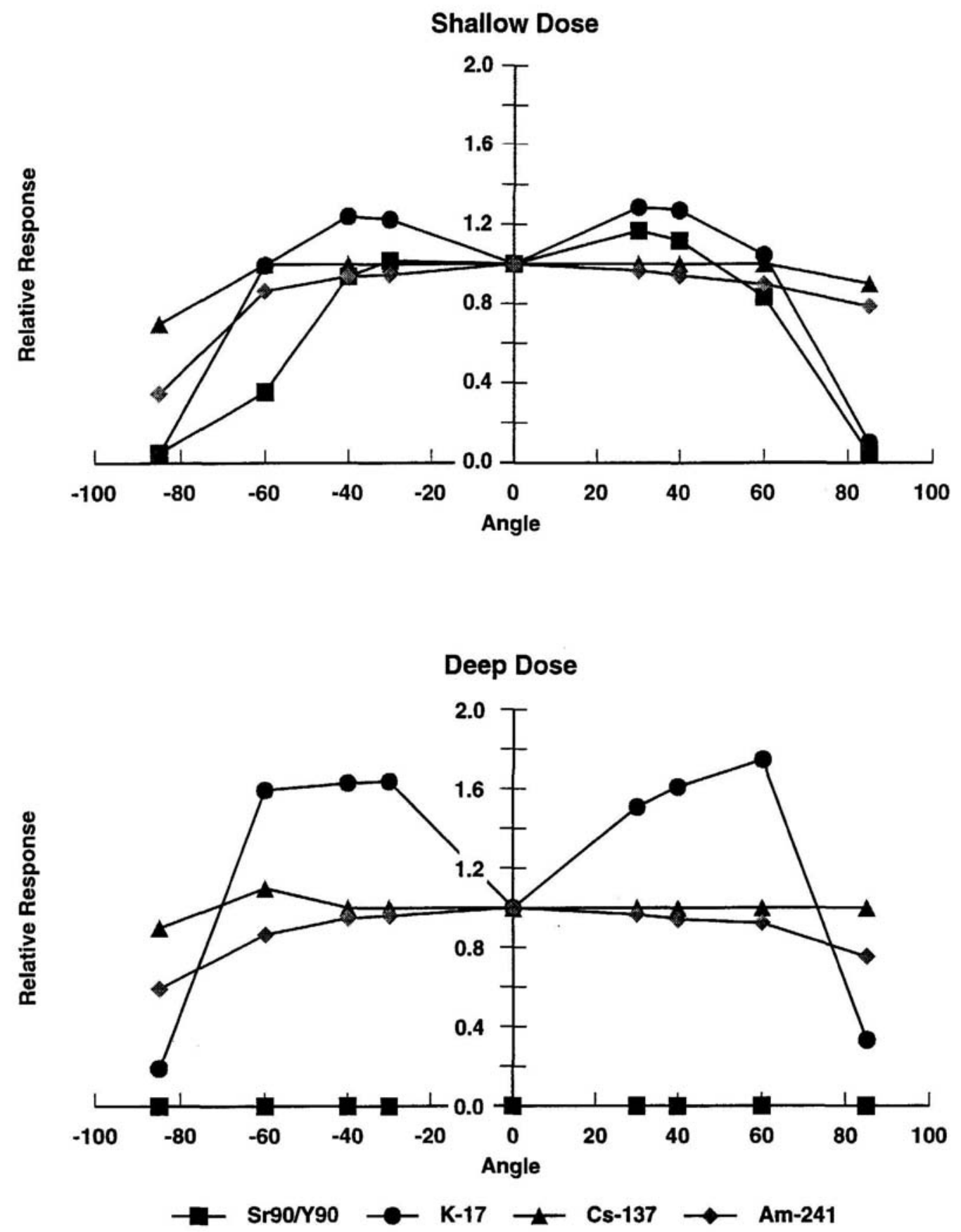

39502006.1

Figure 5.12. HSD Angular Response - Vertical Rotation 


\section{Shallow Dose}

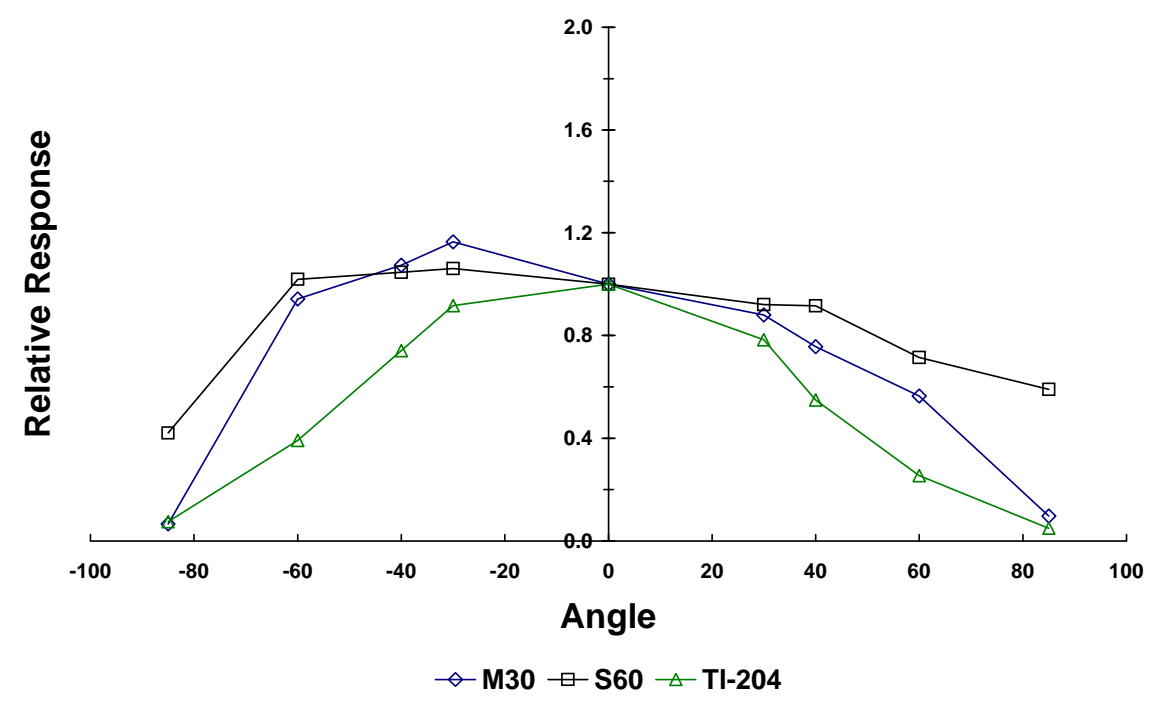

\section{Deep Dose}

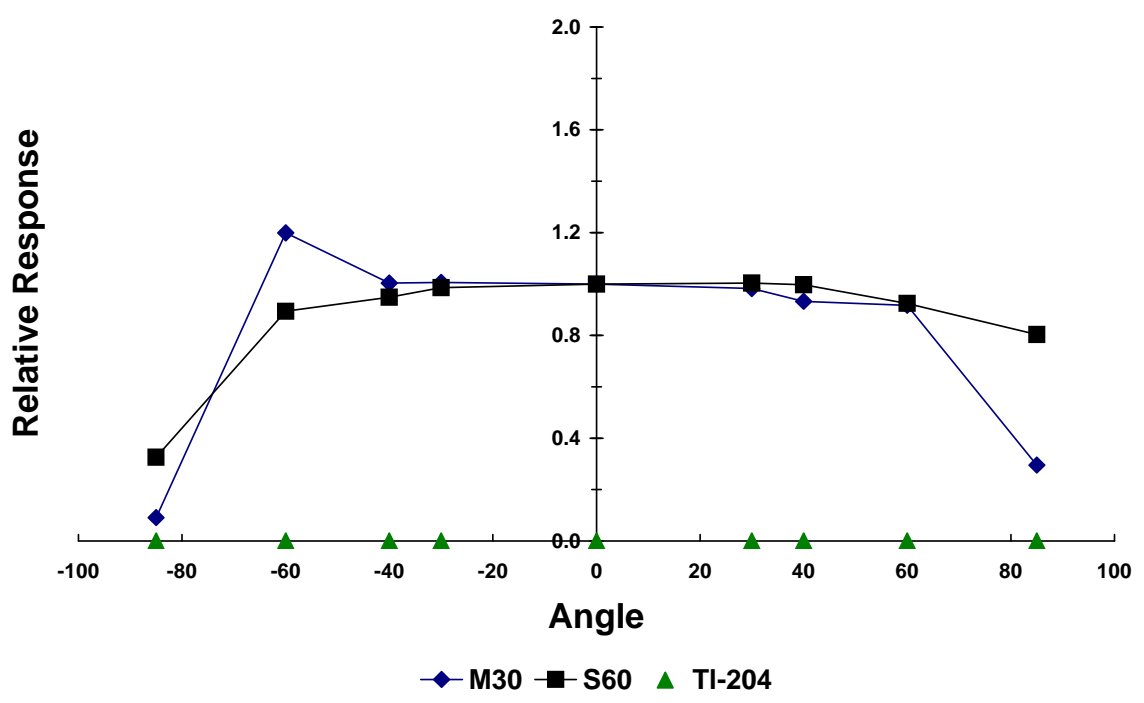

Figure 5.13. HSD Angular Response - Horizontal Rotation 


\section{Shallow Dose}

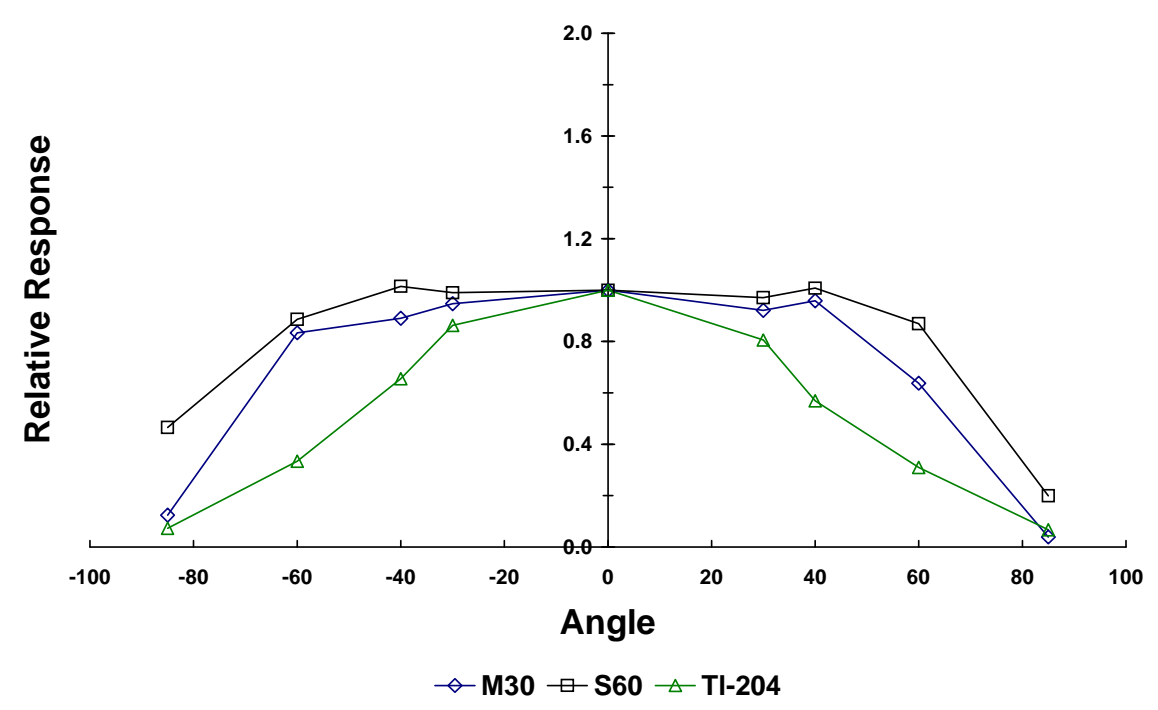

Deep Dose

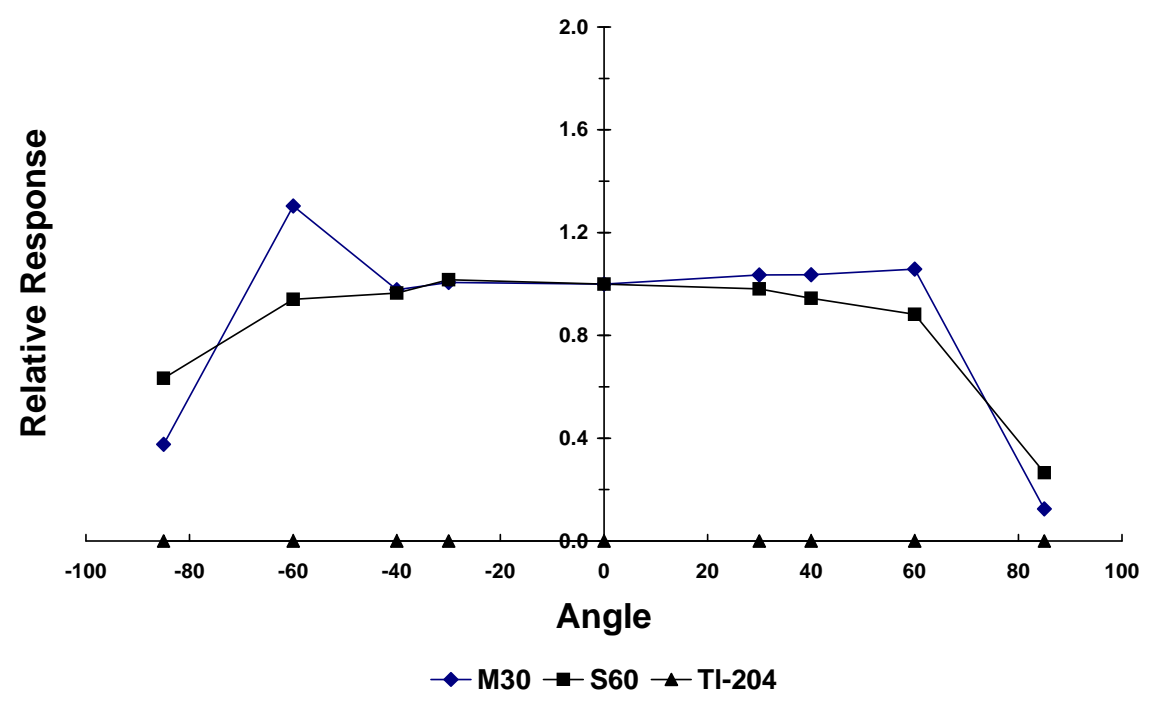

Figure 5.14. HSD Angular Response - Vertical Rotation 


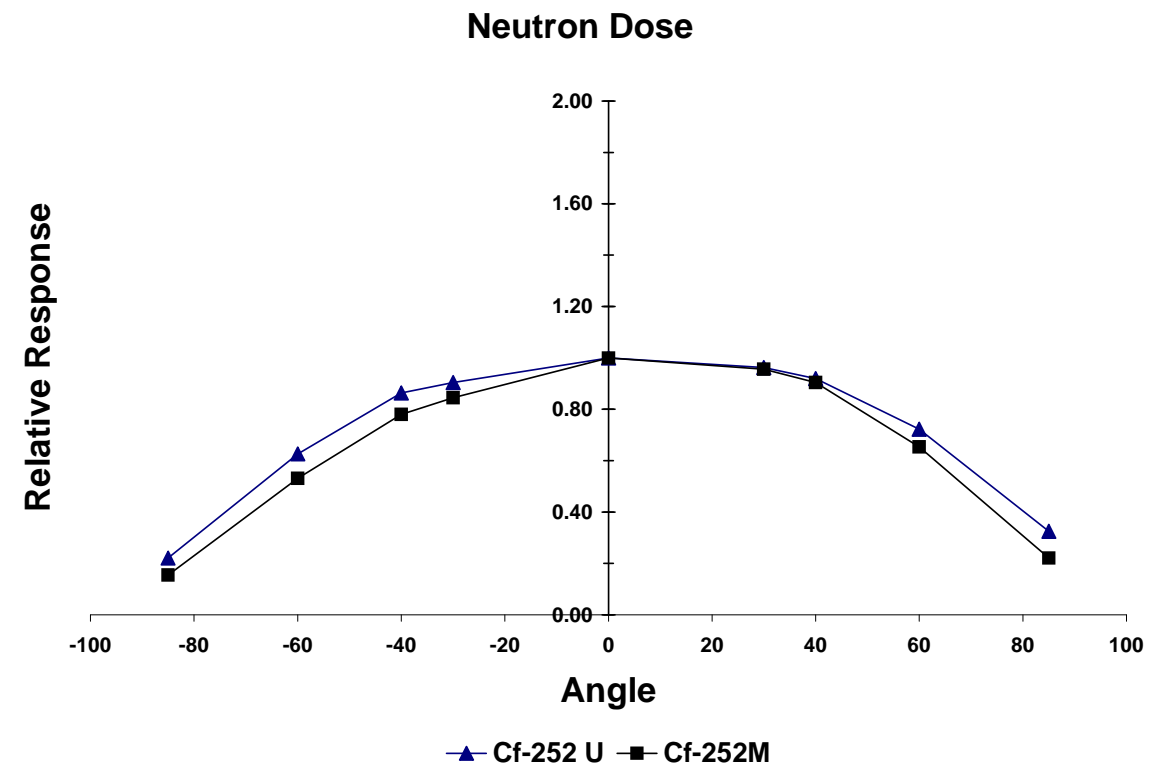

Figure 5.15. HSD Neutron Angular Response - Horizontal Rotation

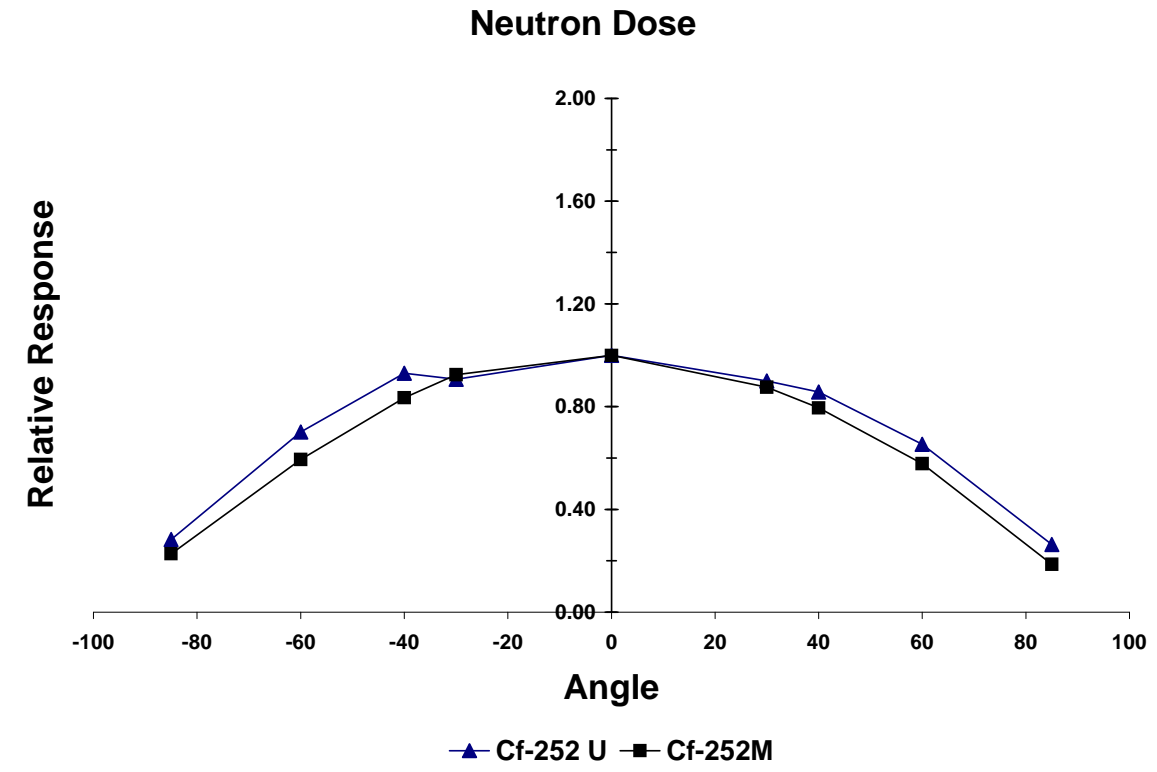

Figure 5.16. HSD Neutron Angular Response - Vertical Rotation 


\subsubsection{Lower Limit of Detection}

The lower limit of detection (LLD) has been calculated for the HSD for monthly, quarterly and annual exchange periods in a variety of studies using either of the two methods given in the DOELAP performance test standard DOE/EH-0027 (DOE 1986a). ${ }^{\text {(a) }}$ These studies used open audit dosimeter data, DOELAP performance test data, and data from dosimeters prepared specifically for the purpose of conducting an LLD study. A composite of the results from these studies is presented in Table 5.12. The symbols $\mathrm{H}_{\mathrm{p}}(0.07), \mathrm{H}_{\mathrm{p}}(3), \mathrm{H}_{\mathrm{p}}(10)_{\gamma}$ and $\mathrm{H}_{\mathrm{p}}(10)_{\eta}$ represent the algorithm calculated shallow personal dose equivalent, eye personal dose equivalent deep personal dose equivalent from photons and deep personal dose equivalent from neutrons respectively. The LLDs for eye personal dose equivalent were not calculated because the delivered eye personal dose equivalent was not given in the irradiations used. However, LLDs for eye dose are expected to be similar to those calculated for deep dose because the variability in eye dose element readings for control dosimeters as well as exposed dosimeters are similar to the variability of the deep dose element.

Table 5.12 Calculated LLDs (in mrem) for the HSD

\begin{tabular}{|c|c|c|c|c|c|c|c|}
\hline $\begin{array}{l}\text { Exchange } \\
\text { Frequency }\end{array}$ & $\begin{array}{l}\text { DOELAP } \\
\text { Category }\end{array}$ & Parameter & $\mathrm{H}_{\mathrm{p}}(0.07)$ & $\mathrm{H}_{\mathrm{p}}(3)$ & $\mathrm{H}_{\mathrm{p}}(10)_{\gamma}$ & $\begin{array}{c}\mathrm{H}_{\mathrm{p}}(10)_{\eta} \\
\text { (mod cal) }\end{array}$ & $\begin{array}{c}\mathrm{H}_{\mathrm{p}}(10)_{\eta} \\
\text { (bare cal) }\end{array}$ \\
\hline \multirow[t]{6}{*}{$\mathrm{M}$} & Controls & $\mathrm{L}_{\mathrm{C}}$ & 3.8 & 3.1 & 3.1 & $*$ & $*$ \\
\hline & $\begin{array}{l}\text { IIIA (X-ray- } \\
\text { general) }\end{array}$ & $\mathrm{L}_{\mathrm{D}}$ & $*$ & $*$ & $*$ & $*$ & $*$ \\
\hline & IV (Cs-137) & $L_{D}$ & 7.6 & $*$ & 6.2 & $*$ & $*$ \\
\hline & VC (beta-general) & $\mathrm{L}_{\mathrm{D}}$ & $*$ & $*$ & $*$ & * & * \\
\hline & VI (neutron - bare) & $\mathrm{L}_{\mathrm{D}}$ & $*$ & $*$ & $*$ & $*$ & 4.1 \\
\hline & VI (neutron - mod) & $\mathrm{L}_{\mathrm{D}}$ & $*$ & $*$ & $*$ & 0.6 & $*$ \\
\hline \multirow[t]{6}{*}{$\mathrm{Q}$} & Controls & $\mathrm{L}_{\mathrm{C}}$ & 3.4 & 3.0 & 3.0 & 0.4 & 3.0 \\
\hline & $\begin{array}{l}\text { IIIA (X-ray- } \\
\text { general) }\end{array}$ & $\mathrm{L}_{\mathrm{D}}$ & 8.3 & $*$ & 7.3 & $*$ & * \\
\hline & IV (Cs-137) & $\mathrm{L}_{\mathrm{D}}$ & 6.9 & $*$ & 6.1 & $*$ & $*$ \\
\hline & VC (beta-general) & $\mathrm{L}_{\mathrm{D}}$ & 7.3 & $*$ & $*$ & $*$ & $*$ \\
\hline & VI (neutron - bare) & $\mathrm{L}_{\mathrm{D}}$ & * & $*$ & $*$ & $*$ & 6.2 \\
\hline & VI (neutron - mod) & $\mathrm{L}_{\mathrm{D}}$ & $*$ & $*$ & * & 0.9 & $*$ \\
\hline \multirow[t]{6}{*}{ A } & Controls & $\mathrm{L}_{\mathrm{C}}$ & 8.5 & 7.1 & 7.1 & 1.3 & 9.2 \\
\hline & $\begin{array}{l}\text { IIIA (X-ray- } \\
\text { general) }\end{array}$ & $\mathrm{L}_{\mathrm{D}}$ & 22.2 & * & 18.5 & $*$ & $*$ \\
\hline & IV (Cs-137) & $L_{D}$ & 17.2 & $*$ & 14.4 & $*$ & $*$ \\
\hline & VC (beta-general) & $\mathrm{L}_{\mathrm{D}}$ & 18.8 & $*$ & $*$ & $*$ & $*$ \\
\hline & VI (neutron - bare) & $L_{D}$ & $*$ & $*$ & $*$ & $*$ & 19.0 \\
\hline & VI (neutron - mod) & $\mathrm{L}_{\mathrm{D}}$ & $*$ & $*$ & $*$ & 2.7 & $*$ \\
\hline
\end{tabular}

(a) Letters to HEDP File:

B. A. Rathbone, "LLD Calculations for HSD and HCND Dosimeters," July 9, 1996.

J. J. Fix, "HSD Cf-252 Lower Level of Detection,” June, 2, 1998

B. A. Rathbone, "LLD Calculations for Quarterly HSD," May 20, 1999.

B. A. Rathbone, "LLD Calculations for Annual HSD,” May 20, 1999. 
The $\mathrm{L}_{\mathrm{C}}$ and $\mathrm{L}_{\mathrm{D}}$ values for bare and moderated ${ }^{252} \mathrm{Cf}$ neutrons (shaded) have been updated to reflect the increases in fluence to dose equivalent conversion factors for these sources at PNNL. ${ }^{\text {(a) }}$ The columns for $H_{p}(10)_{\eta}$ (mod cal) and $H_{p}(10)_{\eta}$ (bare cal) pertain to dose results obtained when the algorithm is configured to apply either the moderated or the bare ${ }^{252} \mathrm{Cf}$ calibration factor.

\subsubsection{Environmental Sensitivity}

The HSD is relatively unaffected by normal variations in heat, humidity, and light. The black ABS plastic construction of the holder was chosen to minimize effects of light. However, it is important to protect the dosimeter from environmental extremes because of the potential affect on the dosimeter response. Because the holder color is black, the dosimeter can reach temperatures in excess of $70^{\circ} \mathrm{C}$ when placed in direct sunlight in an unventilated area, such as the dashboard of a car. Data presented by E. Piesch (Oberhofer and Scharmann 1979) indicate fade can be as high as $60 \%$ for storage at $70^{\circ} \mathrm{C}$ for 100 days.

Figure 5.17 illustrates results of a study that shows significant fade observed with the HSD from elevated temperatures. In this study, HSDs were prepared using routine procedures (i.e., reader and $80^{\circ} \mathrm{C}$ oven anneals), the cards were exposed to $500 \mathrm{mR}$ of ${ }^{60} \mathrm{Co}$ gamma radiation, loaded into holders, and maintained at $80^{\circ} \mathrm{C}$ in an oven for selected time periods of up to 28 days. Fading in excess of $50 \%$ was observed.

While the HSD is unaffected by light, the bare card used in the dosimeter is sensitive to light. This sensitivity is due to the sensitivity of the TL phosphors and Teflon ${ }^{\circledR}$ encapsulation to visible and ultraviolet (UV) light. At ordinary indoor lighting levels, LiF:Mg,Ti response to light is negligible whereas the Teflon $^{\circledR}$ encapsulation is unacceptably large for a small percentage of the elements. Acceptance testing of the 8825 and 8816 card types for production use shows a mean light-induced signal of less than $5 \mathrm{mR}$ with batch-to-batch fluctuations evident when exposed for 2 hours to UV-filtered fluorescent room lighting at a level of 300 lux, which is representative of routine operating conditions. ${ }^{\text {(b) }}$ Approximately $0.5 \%$ of the cards tested exhibit a response in excess of $20 \mathrm{mR}$ on at least one chip when exposed under these conditions, with extremes greater than $200 \mathrm{mR}$ having been observed. For this reason, the card should never be removed from the holder while in the field.

\subsubsection{Fading}

The fade corrections used for TLD 600 and TLD 700 elements in the HSD are described in Section 5.3.6.2. In general, fading is less than 15\% per year for beta-

(a) B. A. Rathbone, "Re-evaluation of Neutron LLDs for HSD with New Neutron Calibration Factors,” August 13, 2009, Letter to HEDP file.

(b) Procedure 200.3.10. “Acceptance Testing of Type 8825 and 8816 Cards,” PNL-MA-841, Hanford External Dosimetry Project Procedures Manual. 
gamma dose and less than 30\% per year for neutron dose. Default fade corrections are applied, based on the assumption that a dosimeter was exposed mid cycle. This type of fade correction produces accurate results for acute exposures at mid cycle and for chronic exposures. However, when all of the exposure is received at the beginning or end of the exposure cycle, errors are introduced by use of default fade corrections. The worst case error in reported beta-gamma dose for a single acute exposure at the beginning or end of the exposure cycle is $-5 \%$ and $+10 \%$ respectively for an annual badge. The worst case error in reported neutron dose for a single acute exposure at the beginning or end of the exposure cycle is $-10 \%$ and $+30 \%$ respectively for an annual badge.

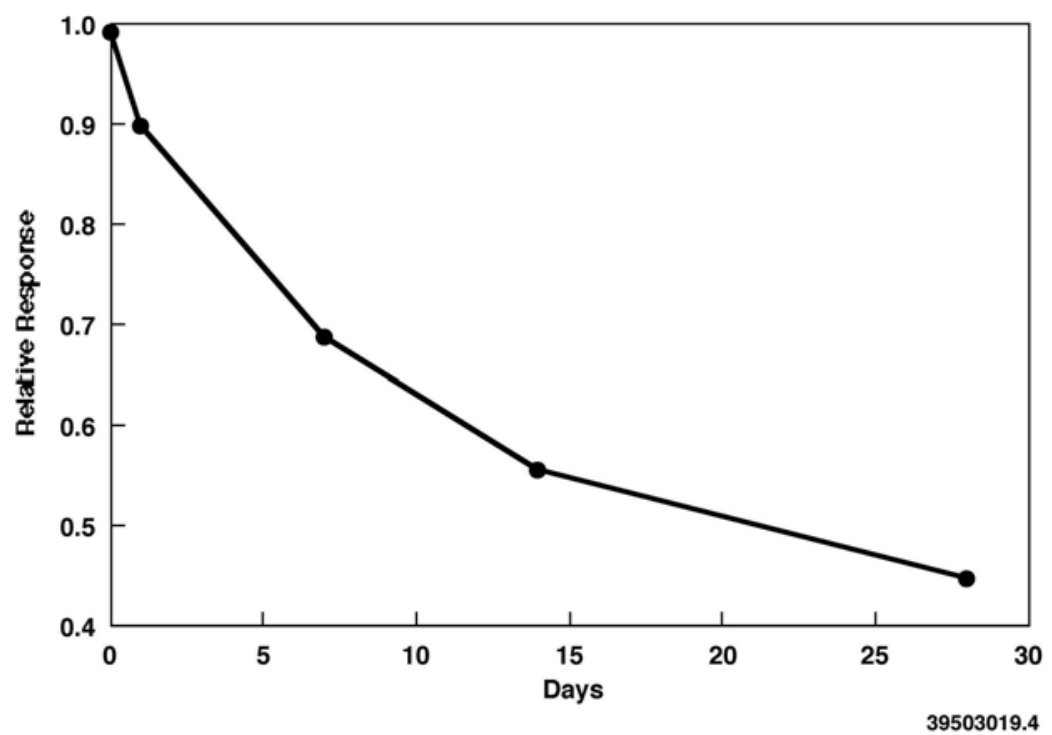

Figure 5.17. HSD Fading at $80^{\circ} \mathrm{C}$

\subsection{Hanford Combination Neutron Dosimeter}

The HCND is used to measure $H_{p}(0.07), H_{p}(3), H_{p}(10)_{\gamma}$ and $H_{p}(10)_{\eta}$ for Hanford employees working in beta, photon, and neutron radiation fields. The dosimeter consists of the following components:

- a beta-photon 8825 TLD

- an albedo neutron 8816 TLD

- a clear plastic HCND holder

The beta/photon TLD and the albedo neutron TLD are known commercially as the Harshaw 8825 and 8816 dosimeters, respectively. In addition to an 8816 card, the 8816 holder contains a pocket to hold two TED foils (CR-39). Because of cost considerations and performance issues at typically low dose levels, the CR-39 capability is no longer maintained or used at Hanford. Periodic field measurements have demonstrated adequate performance can be obtained with the 8816 TLD albedo neutron dosimeter. Detailed design considerations for the HCND are provided by Brackenbush, Baumgartner and Fix (1991) and Endres et al (1996). The HCND holder is used to retain the 8816 and 8825 TLDs, along with an optional Personnel Nuclear Accident Dosimeter (PNAD), as shown in Figure 5.18. The HCND holder is made of polyethylene and the density 
thickness is approximately $95 \mathrm{mg} / \mathrm{cm}^{2}$ in the area behind the 8816 dosimeter and $170 \mathrm{mg} / \mathrm{cm}^{2}$ in the area behind the 8825 dosimeter. The HCND without PNAD is shown in Figure 5.19. Photographs of the HCND are included at the end of Chapter 6. The PNAD is discussed in Section 5.10. The 8825 beta-photon dosimeter is identical to the HSD described in Section 5.4, with the single exception that a TLD-700 phosphor is used in position 4 instead of a neutronsensitive TLD-600 phosphor. This is done to allow for better shallow, eye, and deep dose performance while in a neutron field. Characteristics of the albedo neutron 8816 TLD are described in this section. An illustration of the 8816 albedo neutron dosimeter is shown in Figure 5.20

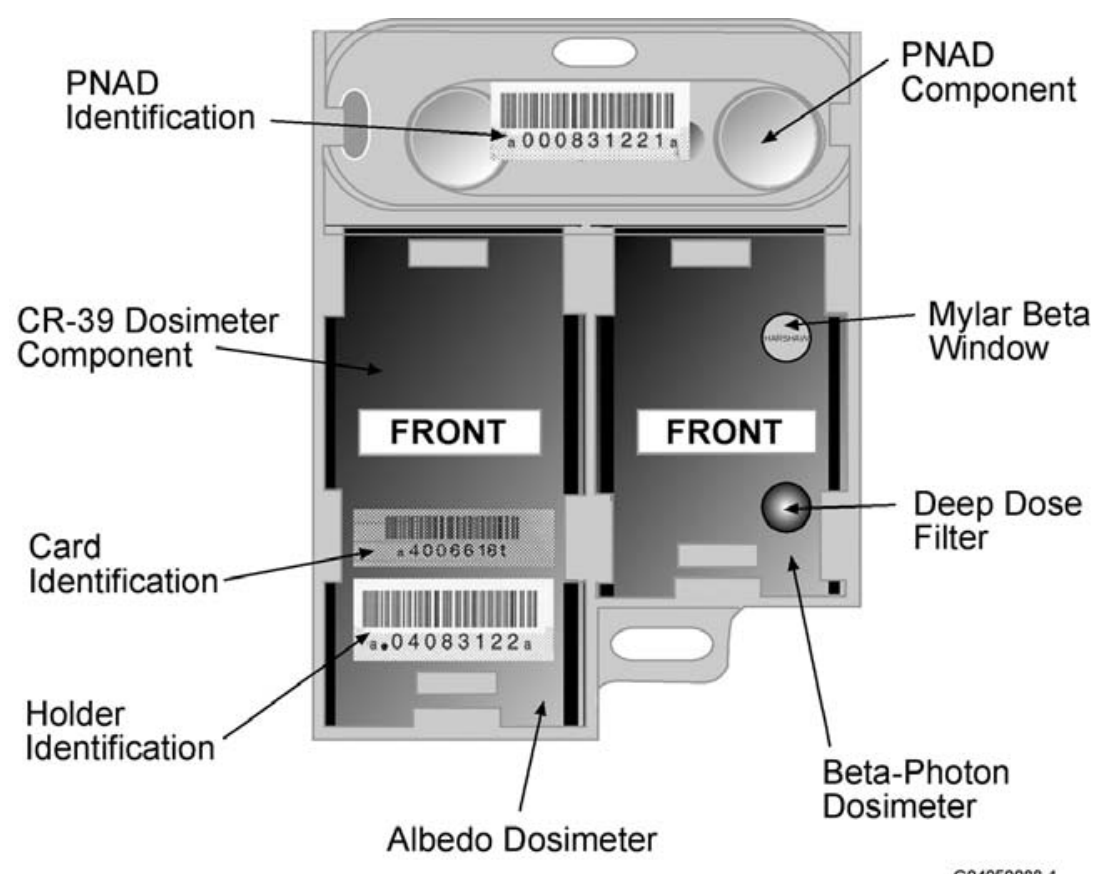

Figure 5.18 Hanford Combination Neutron Dosimeter with PNAD

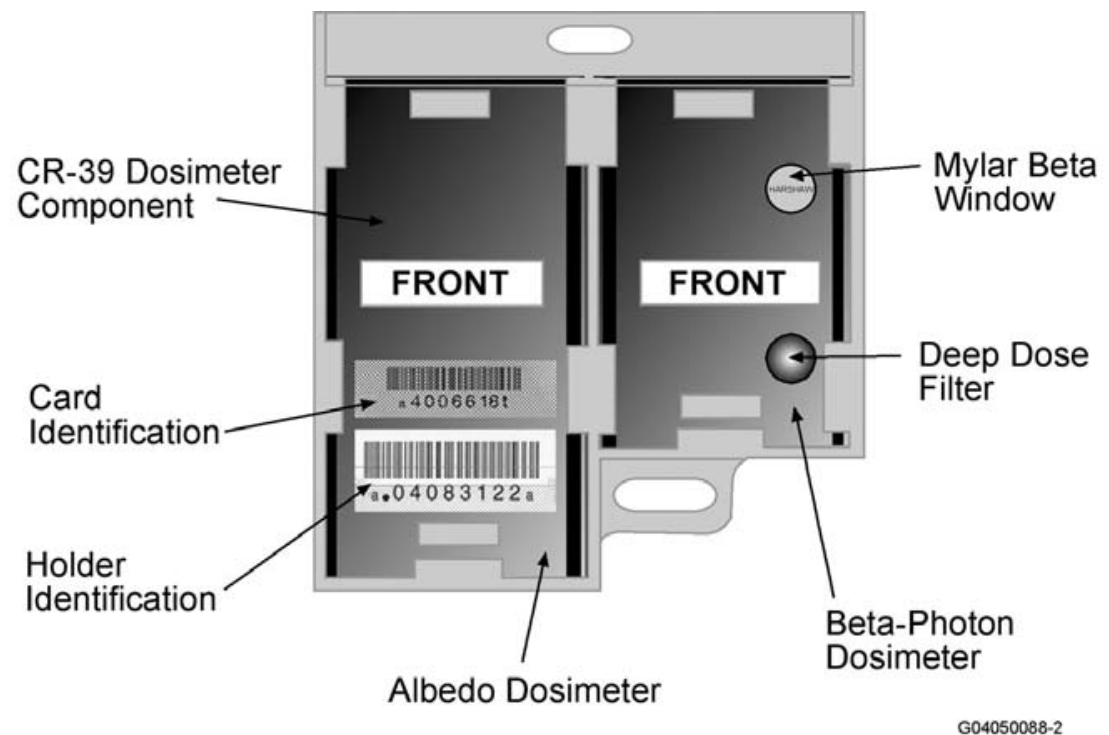

Figure 5.19 Hanford Combination Neutron Dosimeter without PNAD 


\subsubsection{General Features}

The 8816 albedo neutron dosimeter contains three TLD-600 phosphors and one TLD-700. All four positions have nearly the same beta and photon radiation response because of the use of very similar (i.e., similar atomic number) filter materials and thicknesses over each dosimeter position. Three different filter configurations are used for each of the TLD-600 phosphors, as follows:

- cadmium filter on front and tin filter on the back

- tin filter on front and cadmium filter on the back

- tin filters on front and back.

For the single TLD-700 phosphor, tin filters are used on the front and the back. The dosimeter card has 0.38-mm phosphors in all four positions. The tin and cadmium filters have nearly equivalent mass density values of $464 \mathrm{mg} / \mathrm{cm}^{2}$ and $461 \mathrm{mg} / \mathrm{cm}^{2}$, respectively, based on a density of $7.275 \mathrm{~g} / \mathrm{cm}^{3}$ for tin and 8.608 $\mathrm{g} / \mathrm{cm}^{3}$ for cadmium. An additional $80 \mathrm{mg} / \mathrm{cm}^{2}$ of ABS plastic is present in all filter locations.

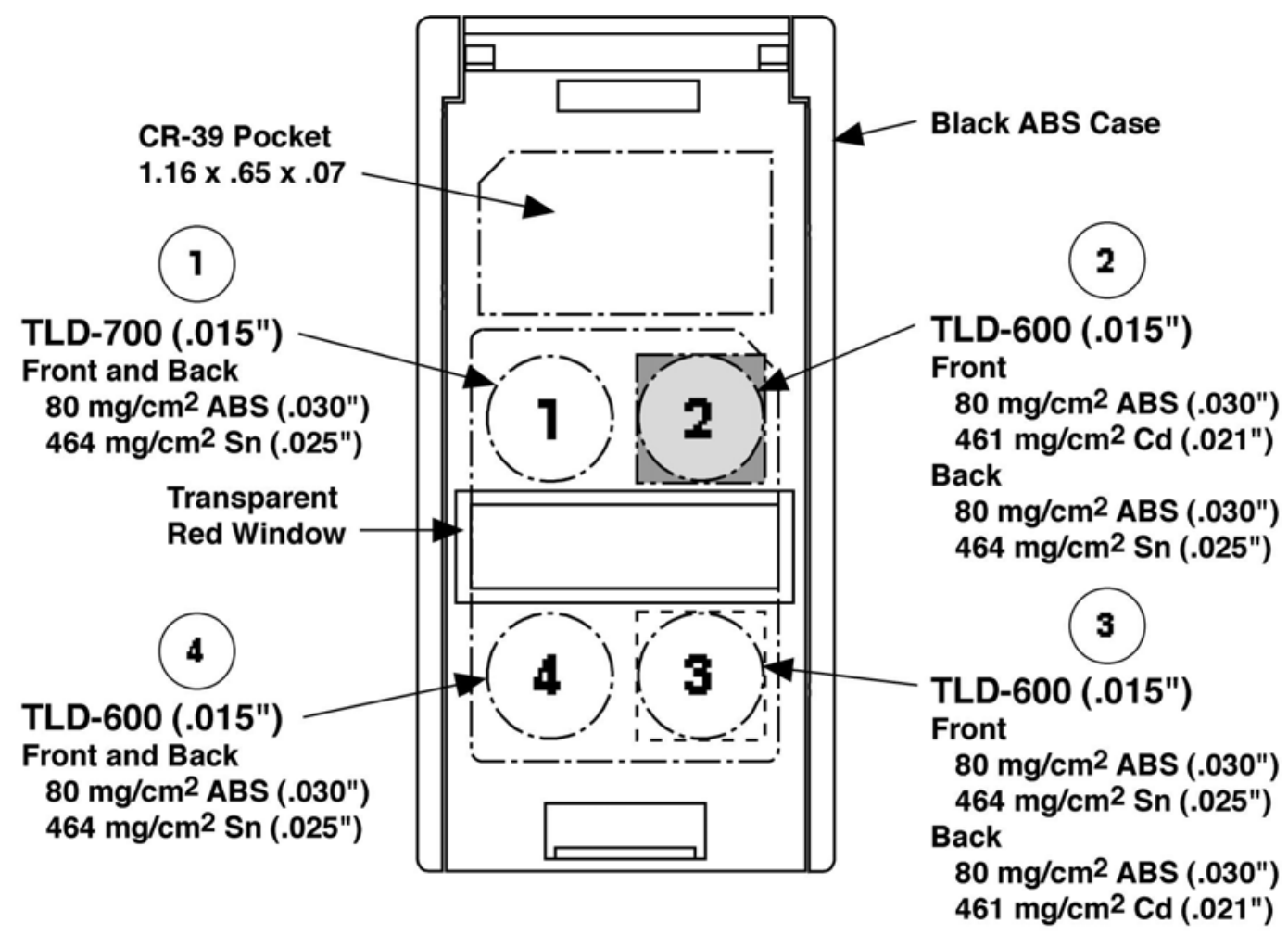

\section{Hanford TL Albedo Neutron Dosimeter}

39502026.4

Figure 5.20 8816 Albedo Neutron Dosimeter 


\subsubsection{Dosimeter Assignment and Processing Protocol}

HCND cards and holders must routinely satisfy the same QC checks upon issue to, and receipt from, field dosimetry organizations as described in Section 5.4.2 for the HSD. Additional steps are involved to load the 8825 beta-photon and 8816 albedo neutron dosimeters together in the combination holder for issuance as a package. Because it is possible to successfully snap an 8816 holder shut with a TLD card loaded backward, 8816 holders are visually inspected after loading to verify proper loading. Otherwise, there are no differences between preparing this dosimeter for assignment and the subsequent TLD processing protocol.

\subsubsection{Algorithm}

The beta-photon 8825 algorithm is identical to the algorithm used with the HSD. The 8816 algorithm calculates only the deep personal dose equivalent from neutrons. All positions of this dosimeter are photon equivalent (i.e., same signal on each phosphor from photon radiation). As such, the TLD-700 phosphor signal in position 1 is used to subtract any photon radiation caused signal from the other positions. There are currently three algorithms that can be used to calculate neutron dose with the 8816 TLD that complies with the 2007 amendment to 10 CFR 835. ${ }^{\text {(a) }}$ These are:

- General Use (californium based)

- $\mathrm{D}_{2} \mathrm{O}$ moderated ${ }^{252} \mathrm{Cf}$

- unmoderated ${ }^{252} \mathrm{Cf}$

The General Use algorithm is used to calculate dose to Hanford workers. The $\mathrm{D}_{2} \mathrm{O}$ moderated ${ }^{252} \mathrm{Cf}$ and unmoderated ${ }^{252} \mathrm{Cf}$ algorithms are used for DOELAP performance testing. They are tailored to these two sources and do not have energy compensating features.

For the General Use algorithm, continuous dose response functions were derived from the element responses and element ratios of the dosimeter when exposed in well characterized neutron radiation fields. To determine the response functions, the dosimeters were exposed on PMMA phantom in AP exposure geometry, to a bare ${ }^{252} \mathrm{Cf}$ source and a ${ }^{252} \mathrm{Cf}$ source with varying diameter polyethylene moderator spheres surrounding the source. Irradiations were performed at the LANL Central Health Physics Calibration Facility. The reference or "delivered" dose was the deep personal dose equivalent from neutrons. The radiation fields were characterized using a combination of MCNP modeling and ROSPEC (proton-recoil based spectrometer) measurements (Seagraves et al, 2008). The General Use algorithm has been shown to provide adequate response for ${ }^{252} \mathrm{Cf}$ fission neutrons moderated by up to six inches of polyethylene, as well as unmoderated $(\alpha, \eta)$ neutrons from ${ }^{241} \mathrm{AmBe}$ sources and neutrons from plutonium contaminated glove boxes.

(a) B. A. Rathbone, “HEDP Dose Calculation Methodology to be Implemented January 1, 2010” September 24, 2009, letter to HEDP file. 


\subsubsection{HCND Element Response to DOELAP Sources}

The relative element response for the beta-photon 8825 TLD is identical to the information presented for the HSD in Table 5.6 except for the chip 4 response to bare and moderated ${ }^{252} \mathrm{Cf}$ irradiations. The difference is due to the fact that chip 4 is TLD-700 in the beta-photon 8825 and TLD-600 in the HSD. For the betaphoton 8825, the shallow personal dose equivalent response and deep personal dose equivalent response of chip 4 to moderated ${ }^{252} \mathrm{Cf}$ is 1.05 and 0.16 , respectively. For unmoderated ${ }^{252} \mathrm{Cf}$ the chip 4 shallow and deep personal dose equivalent responses are 0.77 and 0.05 , respectively. For the 8816 albedo neutron dosimeter, the response for each chip position, for a variety of sources relative to the ${ }^{137}$ Cs response, is shown in Table 5.13. As can be seen from the data, all four chip positions in the 8816 respond approximately the same when exposed to photon or beta radiation thus allowing the use of chip 1 (TLD-700) to subtract beta-photon signals from the chip 2, 3, and 4 readings (TLD-600) to obtain net neutron signal on these chips. Response values for bare and moderated ${ }^{252} \mathrm{Cf}$ are also shown in Table 5.13. These values have been updated to reflect changes in fluence to dose equivalent conversion factors for neutrons and a reassessment of the delivered neutron deep personal dose equivalent rate for the bare and moderated ${ }^{252} \mathrm{Cf}$ sources at PNNL. The updated neutron response values in Table 5.13 reflect a $49 \%$ increase in assessed $\mathrm{H}_{\mathrm{p}}(10)_{\eta}$ for the $\mathrm{D}_{2} \mathrm{O}$ moderated ${ }^{252} \mathrm{Cf}$ source and a $29 \%$ increase for the bare ${ }^{252} \mathrm{Cf}$ source.

Table 5.13 8816 Neutron Dosimeter Element Response to DOELAP Sources

\begin{tabular}{|c|c|c|c|c|c|c|c|c|}
\hline \multirow[b]{3}{*}{$\begin{array}{l}\text { Beam } \\
\text { Code }\end{array}$} & \multicolumn{8}{|c|}{ Element Response Per Unit Delivered Dose Equivalent Relative to ${ }^{137}$ Cs Response } \\
\hline & \multicolumn{4}{|c|}{ Shallow Dose Response ${ }^{a}$} & \multicolumn{4}{|c|}{ Deep Dose Response ${ }^{\mathrm{b}}$} \\
\hline & chip1 & chip2 & chip3 & chip4 & chip1 & chip2 & chip3 & chip4 \\
\hline М30 & 0.03 & 0.02 & 0.02 & 0.02 & 0.06 & 0.06 & 0.06 & 0.06 \\
\hline S60 & 0.15 & 0.15 & 0.14 & 0.13 & 0.16 & 0.16 & 0.15 & 0.14 \\
\hline M150 & 0.39 & 0.41 & 0.38 & 0.39 & 0.37 & 0.39 & 0.37 & 0.37 \\
\hline K16 & 0.02 & 0.02 & 0.02 & 0.02 & 0.07 & 0.06 & 0.07 & 0.07 \\
\hline K59 & 0.28 & 0.28 & 0.26 & 0.26 & 0.27 & 0.26 & 0.25 & 0.25 \\
\hline H150 & 0.63 & 0.63 & 0.63 & 0.63 & 0.63 & 0.63 & 0.63 & 0.63 \\
\hline${ }^{90} \mathrm{Sr} /{ }^{90} \mathrm{Y}$ & 0.06 & 0.04 & 0.06 & 0.06 & N/A & N/A & N/A & N/A \\
\hline${ }^{240} \mathrm{Tl}$ & 0.00 & 0.00 & 0.00 & -0.00 & N/A & N/A & N/A & N/A \\
\hline DU & 0.05 & 0.04 & 0.05 & 0.05 & N/A & N/A & N/A & N/A \\
\hline${ }^{137} \mathrm{Cs}$ & 1.00 & 1.00 & 1.00 & 1.00 & 1.00 & 1.00 & 1.00 & 1.00 \\
\hline${ }^{241} \mathrm{Am}$ & 0.29 & 0.31 & 0.27 & 0.28 & 0.28 & 0.30 & 0.26 & 0.27 \\
\hline${ }^{252} \mathrm{Cf} \mathrm{M}$ & $1.16^{\mathrm{c}}$ & $40.46^{\mathrm{c}}$ & $17.15^{\mathrm{C}}$ & $44.97^{\mathrm{C}}$ & $0.12^{\mathrm{d}}$ & $4.05^{\mathrm{d}}$ & $1.72^{\mathrm{d}}$ & $4.51^{\mathrm{d}}$ \\
\hline${ }^{252} \mathrm{Cf} \mathrm{U}$ & $0.84^{\mathrm{c}}$ & $13.40^{\mathrm{C}}$ & $4.52^{\mathrm{C}}$ & $13.85^{c}$ & $0.04^{\mathrm{d}}$ & $0.69^{d}$ & $0.23^{\mathrm{d}}$ & $0.71^{\mathrm{d}}$ \\
\hline
\end{tabular}

a. Response of chips per unit of delivered shallow personal dose equivalent, relative to the ${ }^{137} \mathrm{Cs}$ response.

b. Response of chips per unit of delivered deep personal dose equivalent, relative to the ${ }^{137} \mathrm{Cs}$ response.

c. Delivered shallow personal dose equivalent from ${ }^{252} \mathrm{Cf}$ photons only, was used to calculate these values.

d. Delivered deep personal dose equivalent from ${ }^{252} \mathrm{Cf}$ photons and neutrons was used to calculate these values. 


\subsubsection{Algorithm Bias}

When the General Use algorithm is applied, the $\mathrm{H}_{\mathrm{p}}(10)_{\eta}$ response of the 8816 dosimeter to varying neutron fields is substantially flatter than the HSD which uses a fixed calibration factor. In particular, the 8816 dosimeter has substantially less over response in moderated neutron fields than the HSD. Table $\mathbf{5 . 1 4}$ shows the response of the dosimeter to various sources both in the calibration laboratory (a) and in the field (Conrady et al, 2009). When making field measurements or planning work based on survey data, it should be kept in mind that instruments with isotropic response (e.g. rem ball) that are used to measure ambient dose equivalent in nearly isotropic fields will over estimate effective dose by as much as a factor of two (DOE 1998b) whereas dosimeters worn by an individual or mounted on a phantom will not.

Table 5.14 8816 Neutron Dose Response with General Use Algorithm

\begin{tabular}{||c|c|c|c||}
\hline \multicolumn{4}{|c|}{$8816 \mathrm{H}_{\mathrm{p}}(10)_{\eta}$ Response to Various Sources } \\
(AP exposure geometry) \\
\hline Facility & Source & Distance & Response \\
\hline LANL Calibration & ${ }^{241}$ AmBe bare & $50 \mathrm{~cm}$ & 0.89 \\
\hline LANL Calibration & ${ }^{252} \mathrm{Cf}$ bare & $50 \mathrm{~cm}$ & 1.07 \\
\hline LANL Calibration & ${ }^{252} \mathrm{Cf}+1 "$ poly & $50 \mathrm{~cm}$ & 0.99 \\
\hline LANL Calibration & ${ }^{252} \mathrm{Cf}+2 "$ poly & $50 \mathrm{~cm}$ & 0.96 \\
\hline LANL Calibration & ${ }^{252} \mathrm{Cf}+3 "$ poly & $50 \mathrm{~cm}$ & 1.06 \\
\hline LANL Calibration & ${ }^{252} \mathrm{Cf}+4 "$ poly & $50 \mathrm{~cm}$ & 1.03 \\
\hline LANL Calibration & ${ }^{252} \mathrm{Cf}+5 "$ poly & $50 \mathrm{~cm}$ & 1.00 \\
\hline LANL Calibration & ${ }^{252} \mathrm{Cf}+6 "$ poly & $50 \mathrm{~cm}$ & 0.96 \\
\hline PNNL Calibration & ${ }^{252} \mathrm{Cf}$ bare & $50 \mathrm{~cm}$ & 1.14 \\
\hline PNNL Calibration & ${ }^{241}$ AmBe bare & $50 \mathrm{~cm}$ & 0.91 \\
\hline PFP 234-5 Rm 228A & glove box HC-9B east face ${ }^{(a)}$ & $10 \mathrm{~cm}$ & 1.14 \\
\hline PFP 234-5 Rm 228A & glove box HC-9B south face ${ }^{(\mathrm{a})}$ & $145 \mathrm{~cm}$ & 1.21 \\
\hline PFP 234-5 Rm 228A & glove box HC-9B west face ${ }^{(\mathrm{a})}$ & $10 \mathrm{~cm}$ & 0.92 \\
\hline
\end{tabular}

(a) Heavily contaminated with ${ }^{239} \mathrm{Pu}$ oxide and fluoride compounds.

(a) B. A. Rathbone, "Validation Test Plan And Results for PROCESS_DOSES.C Version 11 (8816 Dose Calculation Software) SCR No. 09-500-03, June 16, 2009 HEDP File. 


\subsubsection{Albedo Response}

Response characteristics of the 8816 TLD component are highly dependent upon the energy of the incident neutron radiation and the geometry of the exposure. An important consideration is the distance between the dosimeter and phantom. To measure this effect, 8816 TLDs on-phantom were irradiated with a bare ${ }^{252} \mathrm{Cf}$ source. The distance from the source to the front face of the phantom, which measured $40 \mathrm{~cm} \times 40 \mathrm{~cm} \times 15 \mathrm{~cm}$ in thickness, was $100 \mathrm{~cm}$. The distance between the dosimeter and the face of the phantom varied from 0 to $10 \mathrm{~cm}$. The measured response is shown in Figure $5.21{ }^{\text {(a) }}$ At a distance of $10 \mathrm{~cm}$, the albedo response is approximately $50 \%$ of the response measured at $1.27 \mathrm{~cm}$.

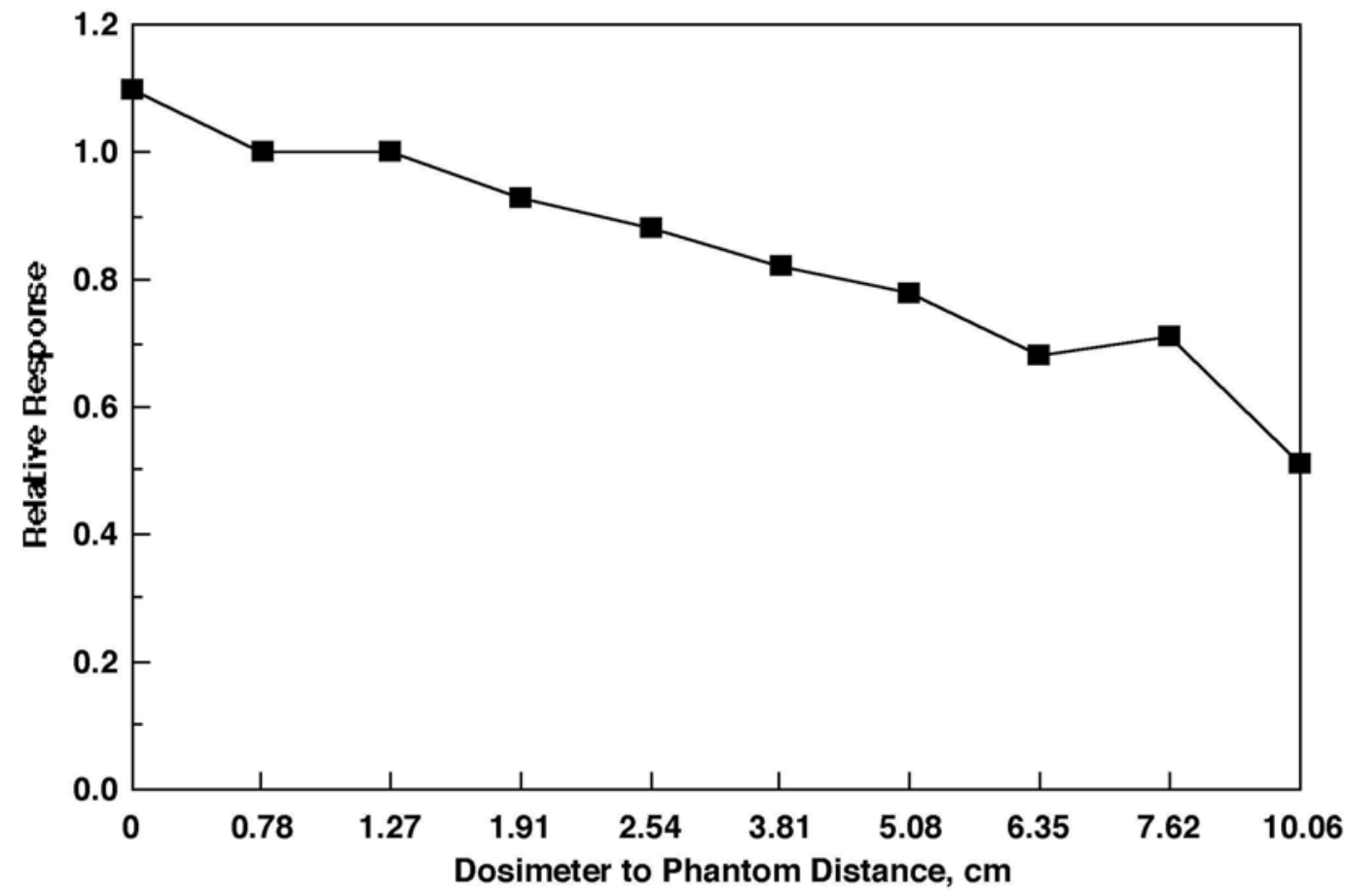

39503019.1

Figure 5.21 Measured Albedo Response of 8816 Neutron Dosimeter

(a) W. V. Baumgartner, “New Badge Response at Different Distances from the Body,” February 3, 1994, letter to HEDP file. 


\subsubsection{Angular Response}

The method described for the HSD was also used to determine the angular response for the HCND except that only one HCND was placed on a phantom at a time to ensure that all active elements were within the central area of the phantom. ${ }^{\text {(a) }}$ The angular response for this dosimeter configured with PNAD, is shown in Tables 5.15 and 5.16, and Figures 5.22 - 5.25. The responses for each source have been normalized to the response at zero degrees for that source. The angular response for neutrons has been updated to reflect the changes in fluence to dose equivalent conversion factors for neutrons, and use of the General Use algorithm for the 8816 dosimeter that was developed based on the new (ICRP 74) conversion factors. ${ }^{(b)}$ The revised values in Table $\mathbf{5 . 1 6}$ are shaded.

Table 5.15 HCND Shallow Dose Angular Response

\begin{tabular}{||c|c|c|c|c|c|c|c|c|c|c|c||}
\hline \hline Source & $\begin{array}{c}\text { Averag } \\
\text { e } \\
\text { Energy } \\
(\mathrm{keV})\end{array}$ & $\begin{array}{c}\text { Axis of } \\
\text { Rotation }\end{array}$ & $-85^{\circ}$ & $-60^{\circ}$ & $-40^{\circ}$ & $-30^{\circ}$ & $0^{\circ}$ & $30^{\circ}$ & $40^{\circ}$ & $60^{\circ}$ & $85^{\circ}$ \\
\hline $\mathrm{K} 16$ & 16 & $\mathrm{H}$ & 0.17 & 0.79 & 1.09 & 1.04 & 1.00 & 1.26 & 1.30 & 1.13 & 0.13 \\
\hline & & $\mathrm{V}$ & 0.08 & 1.01 & 1.10 & 1.07 & 1.00 & 1.11 & 1.11 & 0.80 & 0.06 \\
\hline${ }^{241} \mathrm{Am}$ & 59 & $\mathrm{H}$ & 0.64 & 0.89 & 0.93 & 0.96 & 1.00 & 0.94 & 0.95 & 0.92 & 0.45 \\
\hline & & $\mathrm{V}$ & 0.92 & 1.40 & 1.45 & 1.48 & 1.00 & 1.36 & 1.30 & 1.24 & 0.71 \\
\hline${ }^{137} \mathrm{Cs}$ & 662 & $\mathrm{H}$ & 0.73 & 0.92 & 0.91 & 0.96 & 1.00 & 1.06 & 1.09 & 1.07 & 0.68 \\
\hline & & $\mathrm{V}$ & 0.94 & 1.23 & 1.05 & 1.17 & 1.00 & 1.16 & 0.75 & 0.98 & 0.57 \\
\hline${ }^{90} \mathrm{Sr} /{ }^{90} \mathrm{Y}$ & 931 & $\mathrm{H}$ & 0.05 & 0.22 & 0.86 & 0.62 & 1.00 & 1.20 & 1.37 & 0.77 & 0.50 \\
\hline & & $\mathrm{V}$ & 0.16 & 0.55 & 1.11 & 1.03 & 1.00 & 1.02 & 0.66 & 0.22 & 0.03 \\
\hline
\end{tabular}

Table 5.16 HCND Deep Dose ${ }^{(\mathrm{c})}$ Angular Response

\begin{tabular}{||c|c|c|c|c|c|c|c|c|c|c|c||}
\hline \hline Source & $\begin{array}{c}\text { Average } \\
\text { Energy } \\
(\mathrm{keV})\end{array}$ & $\begin{array}{c}\text { Axis of } \\
\text { Rotation }\end{array}$ & $-85^{\circ}$ & $-60^{\circ}$ & $-40^{\circ}$ & $-30^{\circ}$ & $0^{\circ}$ & $30^{\circ}$ & $40^{\circ}$ & $60^{\circ}$ & $85^{\circ}$ \\
\hline $\mathrm{K} 16$ & 16 & $\mathrm{H}$ & 1.24 & 1.61 & 1.55 & 1.38 & 1.00 & 1.19 & 1.31 & 1.49 & 0.21 \\
\hline & & $\mathrm{V}$ & 0.31 & 1.50 & 1.16 & 1.11 & 1.00 & 1.40 & 1.44 & 1.52 & 0.06 \\
\hline${ }^{241} \mathrm{Am}$ & 59 & $\mathrm{H}$ & 0.44 & 0.81 & 0.89 & 0.92 & 1.00 & 0.79 & 1.04 & 1.22 & 0.65 \\
\hline & & $\mathrm{V}$ & 0.62 & 1.37 & 1.49 & 1.46 & 1.00 & 1.39 & 1.32 & 1.27 & 0.58 \\
\hline${ }^{137} \mathrm{Cs}$ & 662 & $\mathrm{H}$ & 0.78 & 1.01 & 0.98 & 1.02 & 1.00 & 1.04 & 1.06 & 1.08 & 0.78 \\
\hline & & $\mathrm{V}$ & 0.93 & 1.06 & 1.05 & 1.01 & 1.00 & 0.99 & 0.65 & 1.00 & 0.48 \\
\hline${ }^{252} \mathrm{Cf} \mathrm{U}$ & 2100 & $\mathrm{H}$ & 0.19 & 0.700 & 0.96 & n/a & 1.00 & n/a & 0.92 & 0.72 & 0.25 \\
\hline & & $\mathrm{V}$ & 0.33 & 0.78 & 0.93 & n/a & 1.00 & n/a & 0.84 & 0.60 & 0.19 \\
\hline${ }^{252} \mathrm{Cf} \mathrm{M}$ & 550 & $\mathrm{H}$ & 0.10 & 0.47 & 0.69 & n/a & 1.00 & n/a & 0.85 & 0.53 & 0.14 \\
\hline & & $\mathrm{V}$ & 0.16 & 0.52 & 0.83 & n/a & 1.00 & n/a & 0.65 & 0.36 & 0.10 \\
\hline
\end{tabular}

(a) J. J. Fix, “Angular Response of Hanford Personnel Dosimeters,” October 18, 1994, letter to HEDP file.

(b) B. A. Rathbone, “Angular Dependence of Neutron Dose Response for 8816 Dosimeters”, August 10, 2009 letter to HEDP file.

(c) For ${ }^{252} \mathrm{Cf}$ irradiations, neutron component only. 


\section{HCND Shallow Dose Response (Horizontal Rotation)}

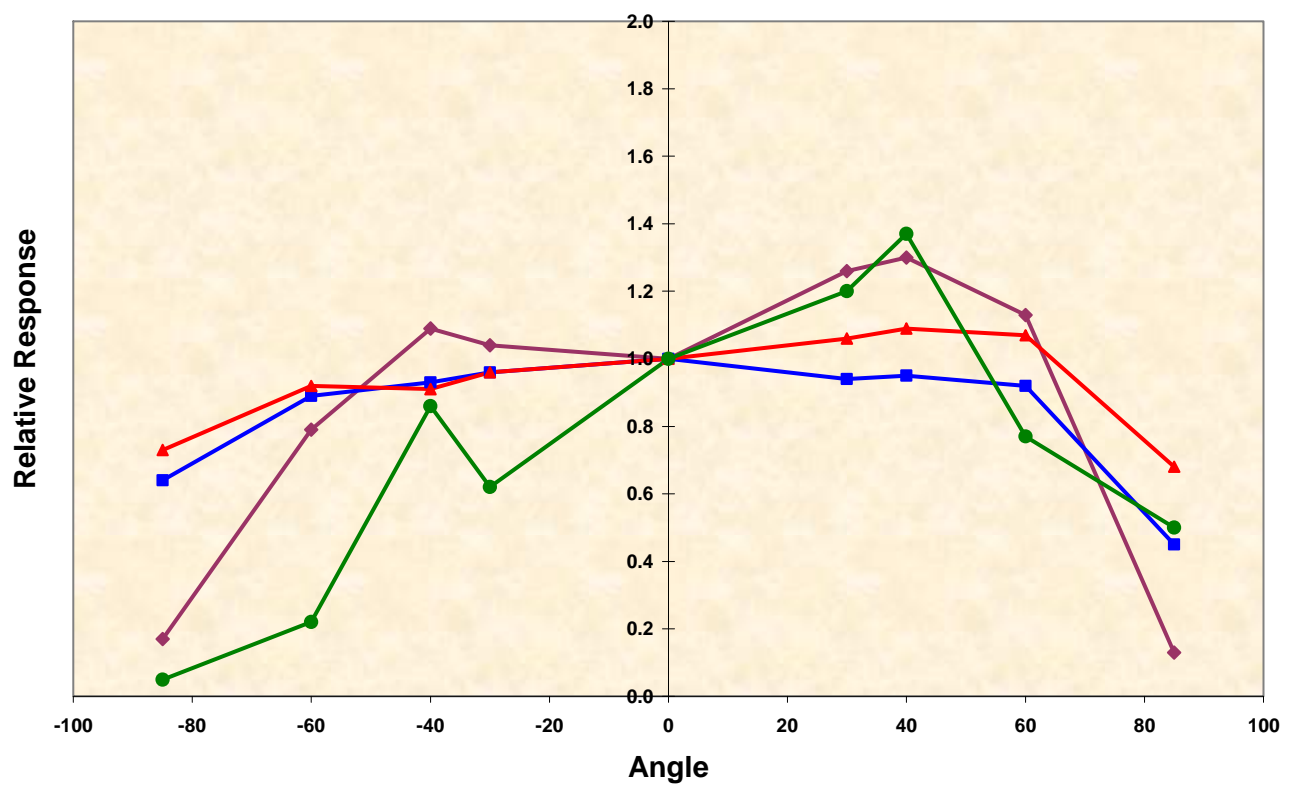

$\leadsto$-K16 $\rightarrow-A m-241 \rightarrow \mathrm{Cs}-137 \multimap-\mathrm{Sr}-90 / \mathrm{Y}-90$

Figure 5.22 HCND Shallow Dose Angular Response - Horizontal Rotation

\section{HCND Shallow Dose Response (Vertical Rotation)}

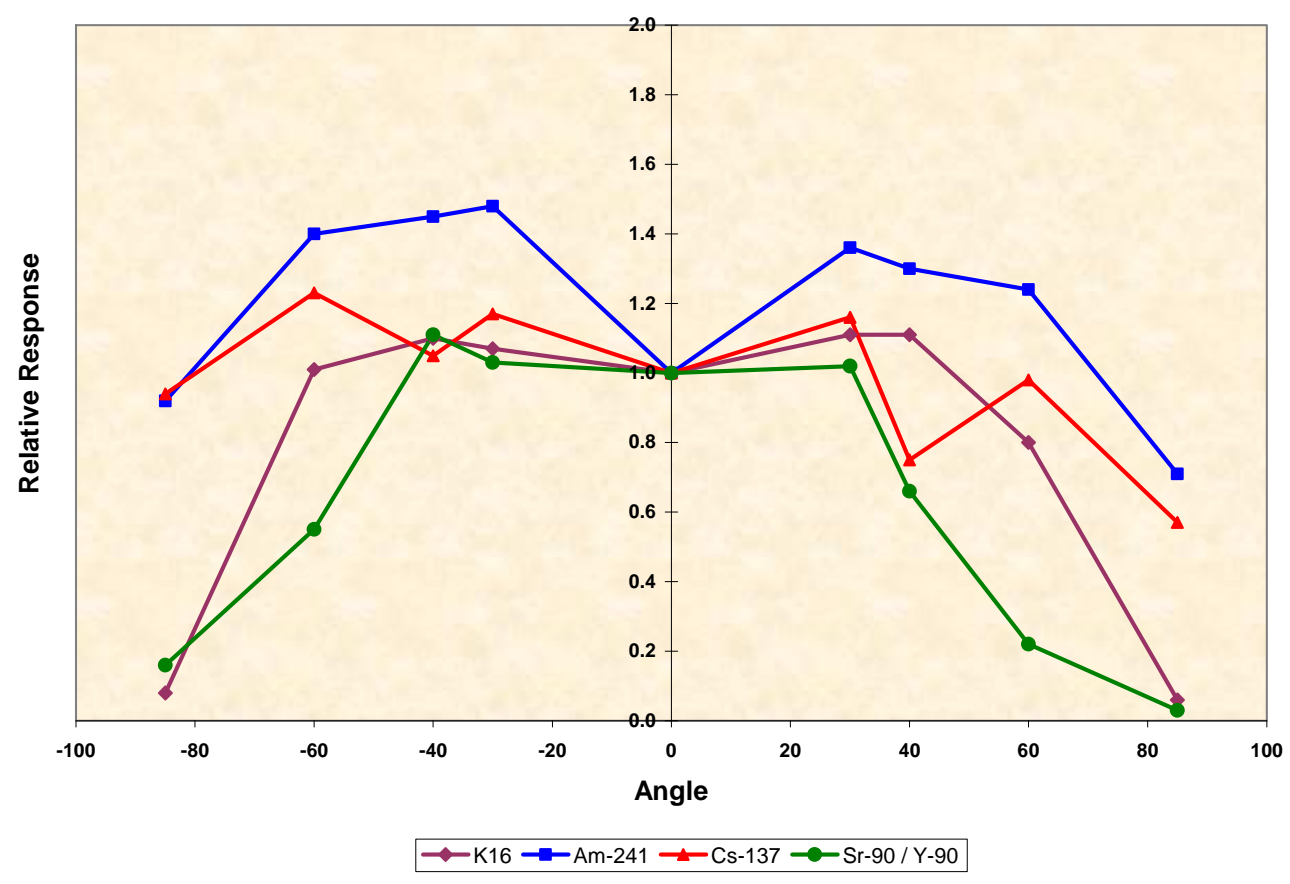

Figure 5.23 HCND Shallow Dose Angular Response - Vertical Rotation 
HCND Deep Dose Response (Horizontal Rotation)

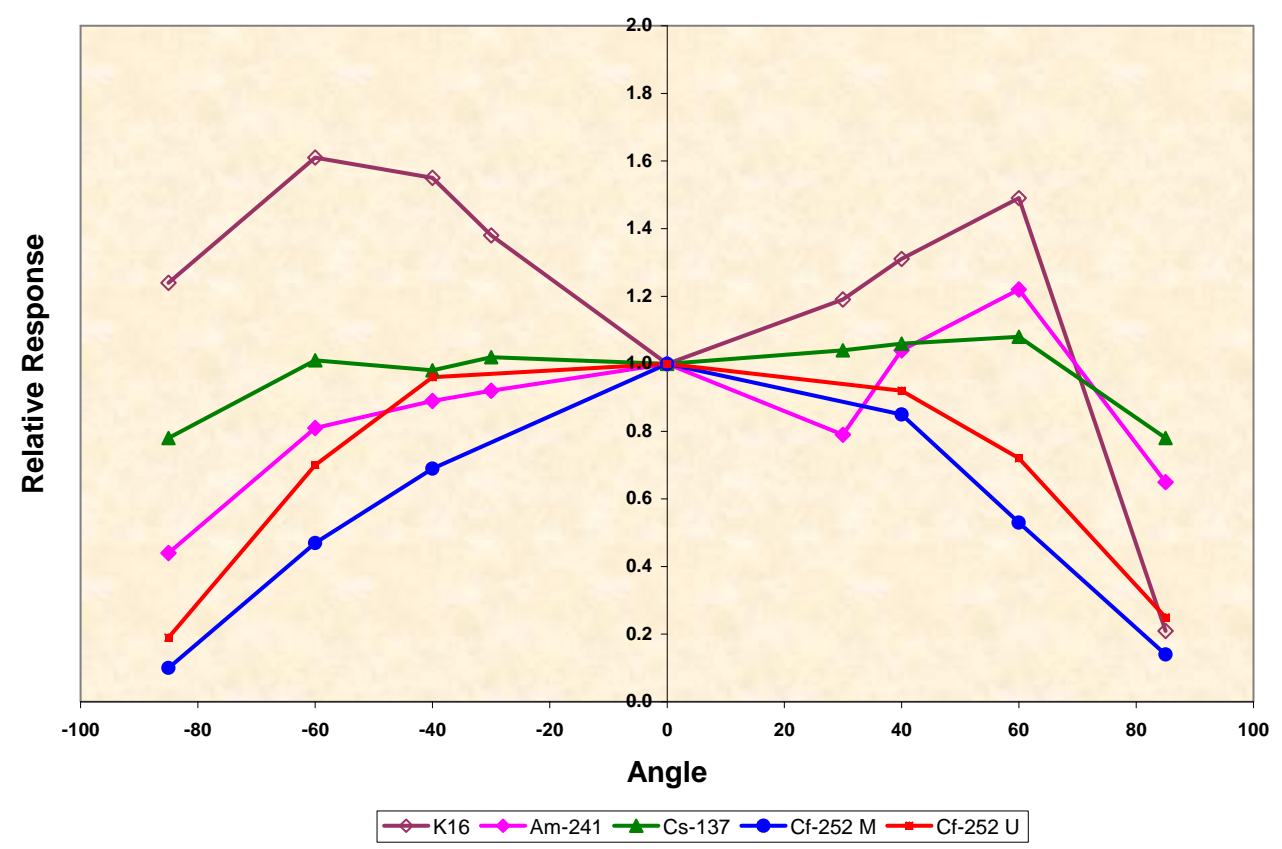

Figure 5.24 HCND Deep Dose Angular Response - Horizontal Rotation

HCND Deep Dose Response (Vertical Rotation)

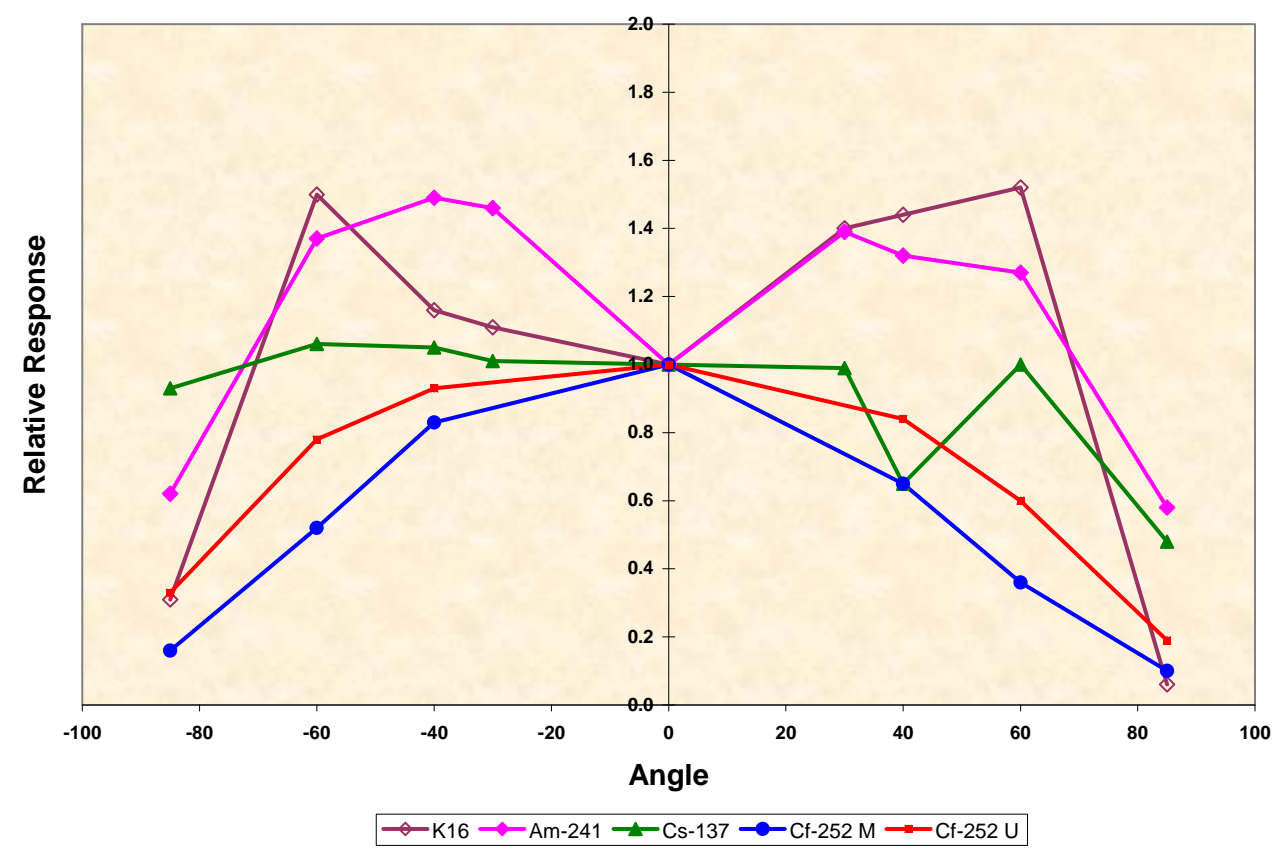

Figure 5.25 HCND Deep Dose Angular Response - Vertical Rotation 


\subsubsection{Lower Limit of Detection}

The LLD has been calculated for the HCND for monthly, quarterly and annual exchange periods in a variety of studies using either of the two methods given in the DOELAP performance test standard DOE/EH-0027 (DOE 1986a). ${ }^{\text {(a) }}$ These studies used unexposed open audit dosimeter data and DOELAP performance test data. A composite of the results from these studies is presented in Table 5.17. The symbols $H_{p}(0.07), H_{p}(3), H_{p}(10)_{\gamma}$ and $H_{p}(10)_{\eta}$ represent the algorithm calculated shallow personal dose equivalent, eye personal dose equivalent, deep personal dose equivalent from photons and deep personal dose equivalent from neutrons respectively. The LLDs for eye dose were not calculated because the delivered eye dose was not given in the irradiations used. However, LLDs for eye dose are expected to be similar to those calculated for deep photon dose because the variability in background readings and dosed readings of the eye dose element in the 8825 dosimeter is similar to that of the deep dose element. The data for $\mathrm{H}_{\mathrm{p}}(10)_{\eta}$ have been updated to reflect changes in fluence to dose equivalent conversion factors for neutrons, and changes in the three dose calculation algorithms used with the 8816 .

Table 5.17 Calculated LLDs (in mrem) for the HCND

\begin{tabular}{|c|c|c|c|c|c|c|c|c|}
\hline $\begin{array}{l}\text { Exchange } \\
\text { Frequency }\end{array}$ & $\begin{array}{l}\text { DOELAP } \\
\text { Category }\end{array}$ & Parameter & $\mathrm{H}_{\mathrm{p}}(0.07)$ & $\mathrm{H}_{\mathrm{p}}(3)$ & $\mathrm{H}_{\mathrm{p}}(10)_{\gamma}$ & $\begin{array}{c}\mathrm{H}_{\mathrm{p}}(10)_{\eta} \\
(\bmod \text { Cf alg) }\end{array}$ & $\begin{array}{c}\mathrm{H}_{\mathrm{p}}(10)_{\eta} \\
\text { (bare Cf alg) }\end{array}$ & $\begin{array}{c}\mathrm{H}_{\mathrm{p}}(10)_{\eta} \\
\text { (General alg) }\end{array}$ \\
\hline \multirow[t]{6}{*}{$\mathrm{M}$} & Controls & $\mathrm{L}_{\mathrm{C}}$ & 3.8 & 3.1 & 3.1 & 0.4 & 1.8 & 1.9 \\
\hline & IIIA (X-ray-general) & $\mathrm{L}_{\mathrm{D}}$ & * & $*$ & $*$ & $*$ & $*$ & $*$ \\
\hline & IV (Cs-137) & $\mathrm{L}_{\mathrm{D}}$ & 7.6 & $*$ & 6.2 & $*$ & $*$ & $*$ \\
\hline & VC (beta-general) & $\mathrm{L}_{\mathrm{D}}$ & $*$ & * & * & * & * & * \\
\hline & VI (neutron-bare) & $\mathrm{L}_{\mathrm{D}}$ & $*$ & $*$ & * & $*$ & 3.5 & 4.0 \\
\hline & VI (neutron-mod) & $\mathrm{L}_{\mathrm{D}}$ & $*$ & $*$ & $*$ & 0.8 & $*$ & 4.1 \\
\hline \multirow[t]{6}{*}{$\mathrm{Q}$} & Controls & $\mathrm{L}_{\mathrm{C}}$ & 3.1 & 2.8 & 2.8 & 0.5 & 2.4 & 2.1 \\
\hline & IIIA (X-ray-general) & $\mathrm{L}_{\mathrm{D}}$ & 7.8 & $*$ & 6.2 & $*$ & $*$ & $*$ \\
\hline & IV (Cs-137) & $L_{D}$ & 6.4 & $*$ & 5.7 & $*$ & $*$ & $*$ \\
\hline & VC (beta-general) & $\mathrm{L}_{\mathrm{D}}$ & 6.5 & $*$ & * & $*$ & $*$ & $*$ \\
\hline & VI (neutron-bare) & $\mathrm{L}_{\mathrm{D}}$ & $*$ & * & * & * & 4.9 & 4.3 \\
\hline & VI (neutron-mod) & $\mathrm{L}_{\mathrm{D}}$ & $*$ & $*$ & $*$ & 1.0 & $*$ & 4.2 \\
\hline & & & & & & & & \\
\hline \multirow[t]{6}{*}{$\mathrm{A}$} & Controls & $\mathrm{L}_{\mathrm{C}}$ & 7.7 & 6.6 & 6.6 & 1.0 & 6.0 & 3.0 \\
\hline & IIIA (X-ray-general) & $\mathrm{L}_{\mathrm{D}}$ & 20.8 & $*$ & 15.1 & $*$ & $*$ & $*$ \\
\hline & IV (Cs-137) & $\mathrm{L}_{\mathrm{D}}$ & 15.7 & $*$ & 13.3 & * & * & $*$ \\
\hline & VC (beta-general) & $\mathrm{L}_{\mathrm{D}}$ & 16.1 & $*$ & $*$ & $*$ & $*$ & $*$ \\
\hline & VI (neutron-bare) & $\mathrm{L}_{\mathrm{D}}$ & $*$ & $*$ & $*$ & $*$ & 12.2 & 6.2 \\
\hline & VI (neutron-mod) & $\mathrm{L}_{\mathrm{D}}$ & $*$ & $*$ & $*$ & 2.0 & $*$ & 6.5 \\
\hline
\end{tabular}

(a) Letters to HEDP file:

B. A. Rathbone, "LLD Calculations for HSD and HCND Dosimeters," July 9, 1996.

B. A. Rathbone, "LLD Calculations for Quarterly HCND," May 20, 1999.

B. A. Rathbone, "LLD Calculations for Annual HCND," May, 1999.

B. A. Rathbone, "LLD Calculations for 8816 Dosimeters," August, 12, 2009 


\subsubsection{Environmental Sensitivity}

The HCND, similar to the HSD, is relatively unaffected by normal variations in heat, humidity, or light. The holder of black ABS plastic was constructed to minimize effects from light. However, it is important to protect the dosimeter from environmental extremes because of the potential effect on the dosimeter response. (See Section 5.4.8)

\subsubsection{Fading}

The fade corrections used for TLD 600 and TLD 700 elements in the HCND are described in Section 5.3.6.2. In general, post irradiation fading is less than $15 \%$ per year for beta-gamma dose and less than $30 \%$ per year for neutron dose. (See Section 5.4.9)

\subsection{Area Dosimetry}

Hanford contractors administer area monitoring programs for the DOE facilities for which they are contractually responsible. Area monitoring programs include routine workplace surveys for external radiation levels, surface contamination levels and airborne radioactivity levels. External radiation monitoring instruments and devices used in area monitoring programs include both fixed and portable instruments that provide real-time indication of radiation levels and passive monitoring devices such as TLDs that provide a retrospective indication of radiological conditions. The focus of this section is to describe the area TLDs used to support area monitoring programs at Hanford. Guidance on area monitoring programs in general is provided in Chapter 6, of the Radiation Protection Programs Guide DOE G 441.1-1C (DOE 2008a) and the DOE Radiological Control Standard DOE-STD-1098-2008 (DOE 2008b).

Area dosimeters are issued by HEDP directly to Hanford contractors without tracking through REX and results are reported directly to Hanford contractors without the use of REX. Area dosimeter results are not stored in REX. Each contractor is responsible for maintaining records of area dosimeter results for their facilities. ${ }^{\text {(a) }}$ Each contractor's records inventory and disposition schedule (RIDS) should treat area dosimetry records in a manner consistent with other workplace surveillance records.

(a) D. E. Bihl, “Minutes of the Hanford Personnel Dosimetry Advisory Committee Meeting Held October 21, 1998.” Copies of HPDAC minutes are retained in Hanford Radiological Records historical file 


\subsubsection{HSD Area Dosimeter}

The HSD area dosimeter is identified by an eight digit holder ID beginning with the digits 06. It is physically identical to the HSD personnel dosimeter, uses the same dose algorithm as the HSD personnel dosimeter, and reports the same operational quantities (personal dose equivalents). Therefore, the angular dependence data, LLD data, fade data, algorithm response data and other data provided for the HSD earlier in this chapter are generally applicable to the HSD area dosimeter as well. Important exceptions are discussed in the paragraphs below.

The LLDs determined for the HSD personnel dosimeter are based on dosimeter response on phantom. To the extent that HSD area dosimeters are used without phantom and under respond as a result (see Tables 5.18 and 5.19), the LLD is increased by a corresponding amount.

The angular dependence data for the HSD personnel dosimeter are based on dosimeter response on phantom. To the extent that HSD area dosimeters are used without phantom, the response at large angles, relative to response at normal incidence may differ from what has been determined for the HSD personnel dosimeter.

It is important to note that natural environmental background is subtracted from area dosimeters in the same manner as with personnel dosimeters. Area dosimeters are intended to measure only radiation from man made sources and provide an indication of potential occupational exposure as would be reported by a personnel dosimeter. Therefore, area dosimeters placed in areas with radiation levels no greater than the average natural background for the Hanford site would be expected to report doses at or near zero mrem.

Reporting thresholds are not applied to area dosimeter results (unlike Personnel dosimeter results). The 8825 algorithm does however apply implicit detection thresholds in its branching logic (e.g. for detection of neutrons, the neutron signal must be at least $20 \%$ of the total signal on chip 4 ).

Since HSDs are calibrated on phantom, ideally, HSD area dosimeters should be placed on phantoms facing the source for the most accurate dose results. However, in many locations where area dosimeters are used this is not practical. As long as the radiation environment consists primarily of beta particles and higher energy photons ( $>100 \mathrm{keV}$ ) the HSD will provide reasonable results even when used without phantom. However, if a substantial part of the exposure is from lower energy photons, the dosimeter may significantly under respond when used without a phantom. ${ }^{\text {(a) }}$ Table $\mathbf{5 . 1 8}$ shows the response when the dosimeter is exposed in air facing the source and facing away from the source. Similar errors can reasonably be expected for dosimeters mounted on gypsum wall board, because of the relatively small mass available for backscatter. The cognizant individual administering the area dosimetry program at each facility should evaluate the need for phantoms, based on a knowledge of the radiation

(a) B. A. Rathbone, “PIC/TLD Response Study,” October, 4, 1995, letter to HEDP file 
environment being monitored, the potential errors involved, and the needed level of accuracy in results.

When mounting an HSD area dosimeter on a wall of any kind, the dosimeter should be facing the interior of the room or hallway in which the dosimeter is being placed. Dosimeters should not be mounted with the Mylar ${ }^{\circledR}$ window facing the wall. This is true even when the primary source of radiation contributing to area dose rates is on the other side of the wall. The rationale for this guidance is as follows: Beta radiation will not be transmitted through the wall; therefore it serves no purpose to have the Mylar ${ }^{\circledR}$ beta window facing the wall. The primary radiations transmitted will be photons $>50 \mathrm{keV}$ and neutrons. In theory, having the metal filters facing the source allows the dosimeter to identify the presence of low energy photons and provide a more accurate result. When the source is in another room, it makes little difference since few low energy photons penetrate most walls. A dosimeter facing away from the wall will provide reasonably accurate shallow and deep dose results for the photons coming through the wall and the dosimeter's back side. To the extent that low energy photons $\underline{\text { do }}$ penetrate the wall, the results will actually be more accurate if the dosimeter has it's backside to the wall (see Table 5.18). If the dosimeter is facing the interior of the room in which it is placed, it has the added advantage of being able to detect and measure any beta radiation or low energy photons that may have been present in the room. It can't do this when facing the wall. In the event of an unexplained high reading, or a suspected loss of radiological control, the ability to assess from dosimeter readings what radiation types may or may not have been present in the rooms in which the dosimeters are placed and to make some statement regarding the accuracy of the reported shallow, eye and deep dose results may be important. The HSD response to neutron radiation when used without phantom is basically independent of orientation.

Table 5.18 HSD Photon Response in Air

\begin{tabular}{||c|c|c|c|c||}
\hline \hline Source & $\begin{array}{c}\text { Average } \\
\text { Energy } \\
(\mathrm{keV})\end{array}$ & $\begin{array}{c}\text { Dosimeter } \\
\text { Orientation }\end{array}$ & $\begin{array}{c}\text { Reported/Given } \\
\text { Shallow Dose }\end{array}$ & $\begin{array}{c}\text { Reported/Given } \\
\text { Deep Dose }\end{array}$ \\
\hline${ }^{137} \mathrm{Cs}$ & 662 & FWD & 0.94 & 0.92 \\
\hline${ }^{137} \mathrm{Cs}$ & 662 & BKWD & 1.01 & 1.01 \\
\hline${ }^{241} \mathrm{Am}$ & 59 & FWD & 0.58 & 0.52 \\
\hline${ }^{241} \mathrm{Am}$ & 59 & BKWD & 0.87 & 0.83 \\
\hline $\mathrm{S} 60$ & 38 & FWD & 0.74 & 0.52 \\
\hline $\mathrm{S} 60$ & 38 & BKWD & 1.22 & 1.31 \\
\hline
\end{tabular}

Although the HSD area dosimeter is not intended for use as a neutron monitoring device, it is sensitive to thermalized neutrons. If neutrons are detected, a neutron dose result will be calculated. However, the calibration factor used to calculate neutron dose is based on calibration of the dosimeter on phantom to a bare ${ }^{252} \mathrm{Cf}$ source (2100 keV avg). If the dosimeter is used with a phantom and exposed to unmoderated fission neutrons, the results will be reasonably accurate. If it is exposed on phantom to moderated (e.g. $550 \mathrm{keV}$ average) neutrons as is typically the case when most neutrons reaching the dosimeter have been scattered several times in hydrogenous shielding and building materials, then the dosimeter may over respond by as much as a factor of 8 . If significant scattering in intervening 
shielding materials (e.g. > 6 inches of water, concrete, or polyethylene) is taking place, then application of a calibration factor based on moderated ${ }^{252} \mathrm{Cf}$ will be more appropriate. If the dosimeter is not placed on a phantom, and the neutron energy spectrum is unmoderated then the dosimeter may under respond by as much as a factor of 40. ${ }^{\text {(a) }}$ If the dosimeter is not placed on a phantom, but the neutron energy spectrum is moderated by intervening shielding (e.g. > 6 inches of water, concrete or polyethylene), then the neutron dose calculated using the default calibration factor will be relatively accurate. If the dosimeter is exposed without a phantom to heavily moderated neutrons (e.g. average energies less than $100 \mathrm{keV}$ ), then the dosimeter may over respond by a factor of 5 or more. Table 5.19 shows the HSD neutron response (reported/given dose) without phantom when the default calibration factor (bare ${ }^{252} \mathrm{Cf}$ ) is used to calculate neutron dose.

Table 5.19 HSD Neutron Response in Air

\begin{tabular}{||c|c|c||}
\hline Source & $\begin{array}{c}\text { Average } \\
\text { Energy } \\
(\mathrm{keV})\end{array}$ & $\begin{array}{c}\text { Neutron } \\
\text { Response } \\
(\mathrm{R} / \mathrm{G})\end{array}$ \\
\hline${ }^{252} \mathrm{Cf}$ Bare & 2130 & 0.027 \\
\hline${ }^{252} \mathrm{Cf} \mathrm{D}_{2} \mathrm{O}(\mathrm{w} / \mathrm{Cd})$ & 550 & 1.07 \\
\hline${ }^{252} \mathrm{Cf} \mathrm{D}_{2} \mathrm{O}$ (w/o Cd) & 550 & 2.66 \\
\hline Concrete Shielded PWR Fuel @ 51 m & 50 & 7.5 \\
\hline
\end{tabular}

As can be seen from the response of the HSD to the $\mathrm{D}_{2} \mathrm{O}$ moderated source without Cd cover compared to its response to the source with Cd cover, the effect of neutrons below $0.4 \mathrm{eV}$ (the Cd cutoff) on dosimeter response in the absence of a phantom is very large. In contrast to their effect on dosimeter response, the effect of neutrons below $0.4 \mathrm{eV}$ on the delivered dose equivalent is quite small. The fluence below $0.4 \mathrm{eV}$ constitutes about $11.5 \%$ of the total fluence emitted from a $\mathrm{D}_{2} \mathrm{O}$ moderated ${ }^{252} \mathrm{Cf}$ source without Cd cover (Schwartz and Eisenhaur, 1982). This same fluence produces only $1.5 \%$ of the total neutron dose equivalent rate. $^{(b)}$ This sensitivity of the HSD to thermal neutrons that do not contribute to dose equivalent is one of the reasons why it is not recommended for use as a neutron dosimeter.

The recommended dosimeter for area monitoring of neutron dose rates is the HCND area dosimeter (described below). The HSD area dosimeter is not recommended for routine monitoring of neutron dose rates unless location specific correction factors based on field measurements with suitable instruments can be applied to the area dosimeter's results and the scatter conditions on which the correction factors are based are not expected to change. An example of one such set of measurements is described below.

(a) B. A. Rathbone, "HSD Neutron Response in Air", May 30, 1999, letter to HEDP file.

(b) L. E. Myers, "HSD Irradiations in Air to Cf-252", May 11, 1999, letter to B. A. Rathbone (included as attachment to May 30, 1999 memo by B. A. Rathbone) 
Measurements made in the vicinity of high burn up PWR fuel stored in NAC-1 casks on a concrete storage pad in the 200 East area before erection of a concrete shield wall indicate that at distances greater than 50 meters, a correction factor (reported/given) of 4 or more may be appropriate for HSD dosimeters used without phantom. ${ }^{\text {(a) }}$ In this case, a large percentage of the neutrons reaching the dosimeter have been scattered one or more times in the shielding of the cask, in the concrete slab, in soil and in the air. Spectrum measurements indicate that the fluence at these distances has an average energy well below $100 \mathrm{keV}$ and a large thermal component. ${ }^{\text {(b) }}$ Spectrum measurements conducted after erection of a concrete shield wall around the fuel indicate an approximate $30 \%$ reduction in the average neutron energy. MCNP modeling of dose rates at selected locations 50 300 meters from the pad indicates that a large fraction of the fluence is from neutrons scattering in air. ${ }^{(c)}$ A comparison of MCNP modeled dose rates with HSD area dosimeter results at nearby locations (50 meters and 80 meters) before and after erection of the wall indicates an approximate $50 \%$ increase to the HSD neutron correction factors (i.e. increase in over response) as a result of the wall. Correction factors at distances greater than 50 meters with the wall in place varied between 5 and $15 .{ }^{(d)}$ The size of correction factor was not always a direct function of distance, most likely because of intervening structures.

Although the above described neutron correction factors were originally determined using dosimeters and instruments calibrated with fluence to dose equivalent conversion factors based on DOE's previous definition of quality factors for neutrons, a re-evaluation of the MCNP modeled dose rates using fluence to dose equivalent conversion factors consistent with the June 8, 2007 amendment to 10 CFR 835 indicates that the correction factors are still valid. ${ }^{\text {(e) }}$ Neutron dose rates without the wall in place increased by a factor of 1.32 on average, when recalculated using ICRP 74 based fluence to dose equivalent conversion factors. The increase was fairly uniform from location to location with a relative standard deviation of only 3.9\%. Neutron dose rates with the wall in place also increased by a factor of 1.32 on average, with a relative standard deviation of only $6.1 \%$. Because neutron doses reported by the HSD area dosimeter have at the same time increased by a factor of 1.29 because of the increase in the calibration factor for bare ${ }^{252} \mathrm{Cf}$, the impact of the $10 \mathrm{CFR} 835$ revision on correction factors for HSD area dosimeters used in the vicinity of spent fuel is negligible.

(a) B. A. Rathbone, “Correction Factors for Neutron Dose Results on Area Dosimeters”, December 31, 2003, Letter to R. L. Hill.

(b) R. I. Scherpelz, “Neutron Measurements on the ISA Pad”, December 9, 2003. Letter report to R. L. Hill.

(c) R. J. McConn and R. I. Scherpelz, "MCNP Estimate of Dose Rates Surrounding the ISA Pad” January 23, 2004, Letter report to R. L. Hill.

(d) B. A. Rathbone, "Correction Factors for Neutron Dose Results on Area Dosimeters Near ISA”, February 12, 2004, Letter report to R. L. Hill.

(e) R. I. Scherpelz, “Re-Evaluation of Neutron Dose Equivalent Rates for the ISA Pad and CSB Vicinity” July 31, 2009, Letter to B. A. Rathbone, HEDP file. 


\subsubsection{HCND Area Dosimeter}

The HCND area dosimeter is physically identical to the HCND personnel dosimeter. The 8825 beta-gamma TLD component is identified by an eight digit holder ID beginning with the digits 036 . The 8816 neutron TLD component is identified by an eight digit holder ID beginning with the digits 046 .

The HCND area dosimeter has the same response characteristics as the HCND personnel dosimeter, uses the same dose algorithms as the HCND personnel dosimeter, and reports the same operational quantities (personal dose equivalents). Therefore, the angular dependence data, LLD data, fade data, algorithm response data and other data provided for the HCND earlier in this chapter are generally applicable to the HCND area dosimeters as well. Important exceptions are discussed in the paragraphs below.

It is important to note that natural environmental background is subtracted from area dosimeters in the same manner as with personnel dosimeters. Area dosimeters are intended to measure only radiation from man made sources and provide an indication of potential occupational exposure as would be reported by a personnel dosimeter. Therefore, area dosimeters placed in areas with radiation levels no greater than the average natural background for the Hanford site would be expected to report doses at or near zero mrem.

Reporting thresholds are not applied to calculated area dosimeter results (unlike personnel dosimeter results). The 8816 algorithm does however apply implicit detection thresholds in it's branching logic (e.g. for detection of neutrons, the neutron signal must be greater than $10 \mathrm{mR}$ equivalent and be at least $10 \%$ of the total signal on each of the TLD 600 chips 2,3, and 4). For the neutron/gamma ratios and moderated neutron energy spectra typically encountered by HCND area dosimeters, this equates to a reporting threshold of 1-2 mrem.

For accurate dose results, the HCND must be placed on a phantom of adequate size and composition. Although current standards in the U.S. specify use of a 40 $\mathrm{cm}$ x $40 \mathrm{~cm}$ x $15 \mathrm{~cm}$ PMMA phantom for neutron dosimeter calibrations and performance testing, investigations have shown minimal differences in albedo dosimeter response between this and the $30 \mathrm{~cm}$ x $30 \mathrm{~cm}$ x $15 \mathrm{~cm}$ PMMA phantom. (McDonald et. al, 1995). Other studies indicate that acceptable phantoms include 9x9 inch polyethylene cylinders, and polyethylene slabs with minimum dimensions of $20 \mathrm{~cm} \times 30 \mathrm{~cm} \times 5 \mathrm{~cm}$, and five gallon water containers (Hankins 1981). The Hankins study indicates that a one gallon water filled container provides marginal response. Studies conducted by HEDP indicate that $25 \mathrm{~cm} \times 25 \mathrm{~cm} \times 34 \mathrm{~cm}$ five gallon water containers provide excellent response (97\%) and PMMA phantoms as small as $20 \mathrm{~cm} \times 20 \mathrm{~cm} \times 15 \mathrm{~cm}$ provide adequate response (83\%). ${ }^{\text {(a) }}$ Although concrete provides some degree of moderation and backscatter, some studies indicate that concrete may not be a suitable phantom material. Albedo neutron dosimeters were shown to significantly under respond when placed on solid concrete walls as thick as eight inches (Hankins 1981). The

(a) B. A. Rathbone, "Impact of Phantom Size and Orientation on 8816 Neutron TLD Response” September 6, 2001, Letter to HEDP file. 
HCND should be centered on the front surface of the phantom. No part of the 8816 dosimeter should be within $10 \mathrm{~cm}$ of the edge of the phantom.

\subsection{HSD Extremity (Wrist/Ankle) Dosimeter}

The HSD Extremity dosimeter is physically identical to the HSD personnel dosimeter. It's intended use is measurement of shallow dose to the upper or lower extremities. It is intended to be worn just above the wrists or ankles with the long axis of the dosimeter parallel to the axis of the forearm or lower leg and has been accredited under DOELAP in this configuration. For accurate results, the dosimeter must be secured to the forearm or lower leg in a manner that leaves as little air space as possible between the dosimeter and the extremity being monitored.

The HSD Extremity dosimeter is labeled in the same manner as the HSD personnel dosimeter and is indistinguishable on the basis of holder ID number (leading digits 00 same as HSD personnel dosimeter). Unlike the Hanford ring dosimeter, it must be assigned as a supplemental dosimeter and therefore is used exclusively as a part of multipacks. About 1000 HSD Extremity dosimeters are used each year at Hanford.

\subsubsection{General Features}

The HSD Extremity dosimeter is identical to the HSD personnel dosimeter and has the same general features described in Section 5.4.1.

\subsubsection{Dosimeter Assignment and Processing Protocol}

The HSD Extremity dosimeter uses the same assignment and processing protocols in the dosimetry lab as the HSD personnel dosimeter. The description provided in Section 5.4.2 is applicable to the HSD Extremity dosimeter as well. However, for assignment to individuals in REX the HSD Extremity dosimeter may be assigned only as a supplemental dosimeter.

\subsubsection{Algorithm}

The HSD Extremity dosimeter uses the same algorithm as the HSD personnel dosimeter described in Section 5.4.3 - 5.4.5. Only the shallow personal dose equivalent results are used for extremity dose of record. The HSD personnel dosimeter algorithm was developed from irradiations on a $30 \mathrm{~cm}$ x $30 \mathrm{~cm} \times 15 \mathrm{~cm}$ slab phantom and delivered dose data based on DOELAP $\mathrm{C}_{\mathrm{x}}$ factors for that phantom. However, PNNL performance testing of the HSD in extremity configuration on a pillar phantom using $\mathrm{C}_{\mathrm{x}}$ factors from the extremity performance test standard HPS N13.32 (HPS, 1996a) indicates acceptable performance as a wrist/ankle extremity dosimeter. ${ }^{\text {(a) }}$ Subsequent DOELAP performance testing has also shown acceptable performance and has lead to DOELAP accreditation of the HSD as an extremity dosimeter.

(a) B. A. Rathbone, “HSD Response on Wrist Phantom,” October 20, 1997, letter to HEDP file. 


\subsubsection{Algorithm Performance}

The results of PNNL performance testing of the HSD as an extremity dosimeter are shown in Table 5.20. Performance is expressed in terms of the bias (B) and standard deviation $(\mathrm{S})$ of performance quotients $\left(\mathrm{P}_{\mathrm{i}}\right)$ for individual dosimeter results (see glossary). The results indicate algorithm biases for individual filtered $\mathrm{X}$-ray techniques, but the overall bias is well within the N13.32 criteria of B < 0.35 .

Table 5.20 HSD Extremity Algorithm Bias

\begin{tabular}{||c|c|c|c||}
\hline Source & $\begin{array}{c}\text { Average } \\
\text { Energy } \\
(\mathrm{keV})\end{array}$ & $\mathrm{B}$ & $\mathrm{S}$ \\
\hline $\mathrm{M} 30$ & 20 & -0.124 & 0.012 \\
\hline $\mathrm{M} 60$ & 34 & 0.085 & 0.021 \\
\hline $\mathrm{M} 100$ & 51 & -0.125 & 0.021 \\
\hline $\mathrm{M} 150$ & 70 & -0.154 & 0.020 \\
\hline $\mathrm{H} 150$ & 117 & -0.069 & 0.033 \\
\hline${ }^{137} \mathrm{Cs}$ & 662 & 0.007 & 0.003 \\
\hline${ }^{204} \mathrm{Tl}$ & 267 & 0.051 & 0.014 \\
\hline${ }^{90} \mathrm{Sr}{ }^{90} \mathrm{Y}$ & 931 & 0.013 & 0.039 \\
\hline
\end{tabular}

\subsubsection{Angular Response}

The angular response of the HSD Extremity dosimeter was measured using the sources, geometry, and method specified in HPS N 13.32 (HPS, 1996a) at the specified angles of $0^{\circ}, \pm 30^{\circ}, \pm 60^{\circ}, \pm 85^{\circ}$ and $180^{\circ}$. (a) The standard specifies that least one source from each category II through IV should be used. For the purpose of this study, two sources from category II (M30 and M100), one source from category III $\left({ }^{137} \mathrm{Cs}\right)$, and two sources from category IV $\left({ }^{90} \mathrm{Sr} /{ }^{90} \mathrm{Y}\right.$ and $\left.{ }^{204} \mathrm{Tl}\right)$ were used. A delivered shallow dose of 1 rem was used for all irradiations. During the irradiations, the HSD was oriented with the Mylar ${ }^{\circledR}$ window at the "top" (see Figure 5.34). With the exception of the $180^{\circ}$ irradiations with beta sources, four dosimeters were used for each angle in vertical rotation and three dosimeters were used for each angle in horizontal rotation. For the $180^{\circ}$ angle no irradiations were performed with the beta sources since the thickness of the extremity phantom exceeds the range of any of the beta particles emitted from the reference sources. The results are shown in Table 5.21 and Figure 5.26 and Figure 5.27.

(a) J. J. Fix, “HSD Extremity Angular Response,” August 4, 1999, letter to HEDP file. 
Table 5.21 HSD Extremity Dosimeter Angular Response

\begin{tabular}{||c|c|c|c|c|c|c|c|c|c|c||}
\hline \hline Source & $\begin{array}{c}\text { Average } \\
\text { Energy } \\
(\mathrm{keV})\end{array}$ & $\begin{array}{c}\text { Axis } \\
\text { of } \\
\text { Rotation }\end{array}$ & $-85^{\circ}$ & $-60^{\circ}$ & $-30^{\circ}$ & $0^{\circ}$ & $30^{\circ}$ & $60^{\circ}$ & $85^{\circ}$ & $180^{\circ}$ \\
\hline $\mathrm{M} 30$ & 20 & $\mathrm{H}$ & 0.09 & 0.67 & 0.94 & 1.00 & 1.09 & 1.07 & 0.15 & 0.01 \\
\hline & & $\mathrm{V}$ & 0.09 & 0.73 & 1.01 & 1.00 & 0.98 & 0.96 & 0.15 & 0.00 \\
\hline $\mathrm{M} 100$ & 51 & $\mathrm{H}$ & 0.73 & 0.78 & 0.93 & 1.00 & 1.04 & 0.99 & 0.59 & 0.05 \\
\hline & & $\mathrm{V}$ & 0.54 & 0.76 & 0.93 & 1.00 & 0.99 & 0.91 & 0.68 & 0.05 \\
\hline${ }^{137} \mathrm{Cs}$ & 662 & $\mathrm{H}$ & 0.89 & 1.02 & 0.99 & 1.00 & 1.00 & 0.98 & 0.79 & 0.42 \\
\hline & & $\mathrm{V}$ & 0.89 & 0.96 & 0.98 & 1.00 & 0.97 & 0.96 & 0.93 & 0.39 \\
\hline${ }^{90} \mathrm{Sr} /{ }^{90} \mathrm{Y}$ & 931 & $\mathrm{H}$ & 0.01 & 0.14 & 0.84 & 1.00 & 1.01 & 0.25 & 0.02 & 0.00 \\
\hline & & $\mathrm{V}$ & 0.02 & 0.18 & 0.90 & 1.00 & 0.93 & 0.19 & 0.05 & 0.00 \\
\hline${ }^{204} \mathrm{Tl}$ & 267 & $\mathrm{H}$ & 0.03 & 0.15 & 0.69 & 1.00 & 0.76 & 0.28 & 0.03 & 0.00 \\
\hline & & $\mathrm{V}$ & 0.02 & 0.15 & 0.65 & 1.00 & 0.74 & 0.18 & 0.02 & 0.00 \\
\hline
\end{tabular}

\section{HSD Extremity Shallow Dose Response (horizontal rotation)}

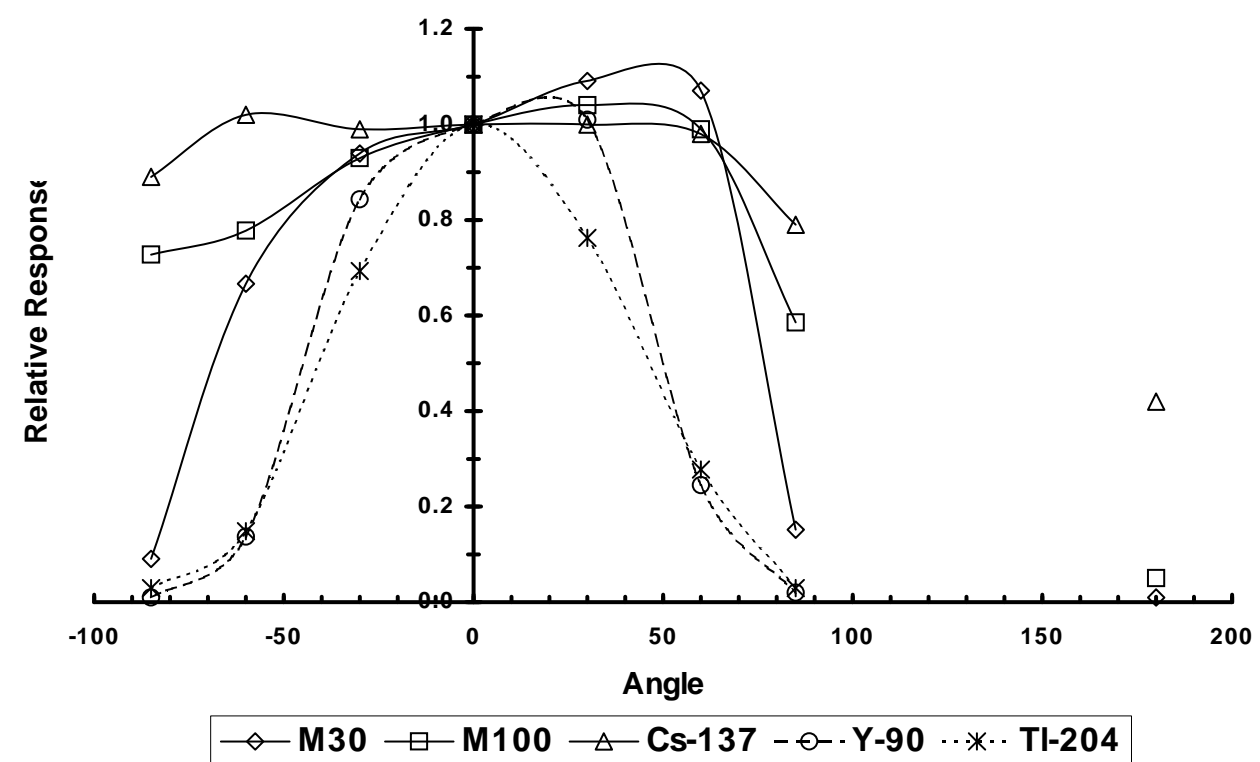

Figure 5.26 HSD Extremity Shallow Dose Angular Response - Horizontal Rotation 


\section{HSD Extremity Shallow Dose Response (vertical rotation)}

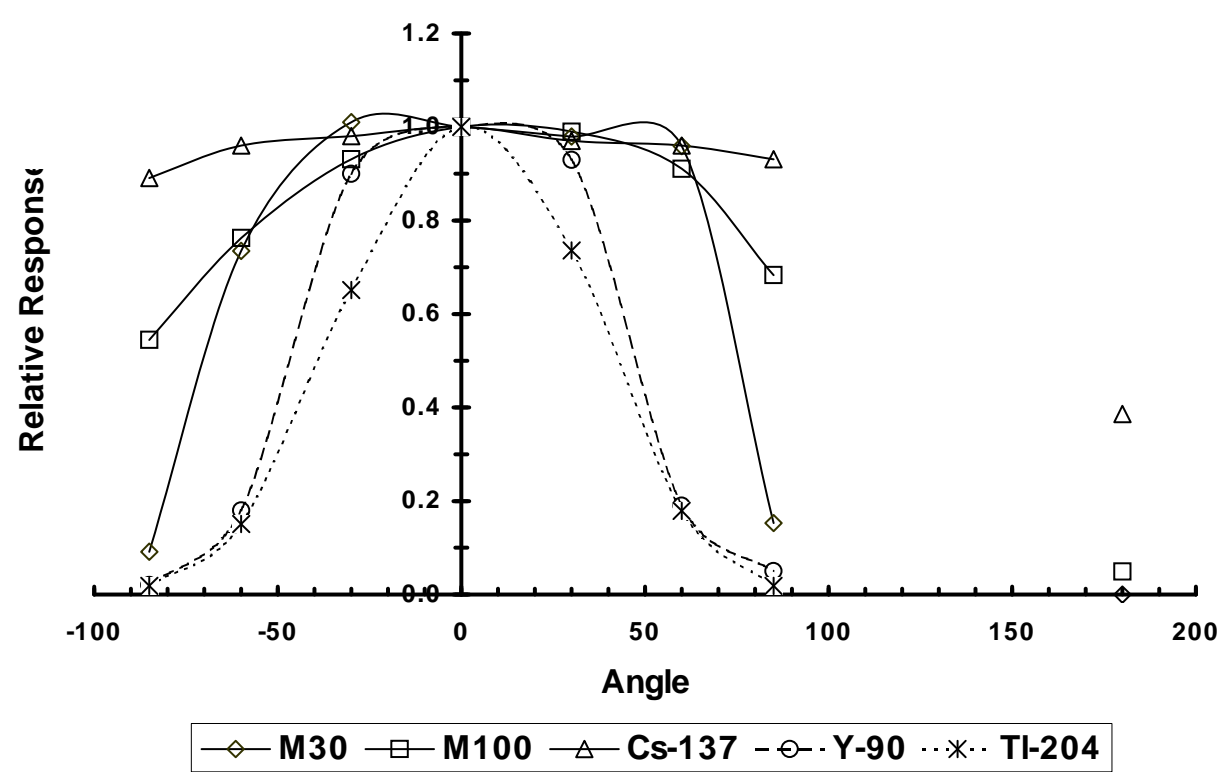

Figure 5.27 HSD Extremity Shallow Dose Angular Response - Vertical Rotation

\subsubsection{Lower Limit of Detection}

The lower limit of detection for the HSD Extremity dosimeter was calculated from exposure data from several beta and photon sources. ${ }^{(a)}$ The analysis was conducted using the procedure described in the Extremity Dosimetry performance standard HPS N 13.32 (HPS, 1996a). The analysis was conducted for the algorithm calculated shallow dose that would be reported and used as the basis for extremity dose of record when the HSD is used as an extremity dosimeter. The dosimeters used for the test had a cycle time of 68 days between anneal and readout which is considered representative of a monthly exchange. Because the HSD Extremity dosimeter's use is limited to multipacks, its exchange frequency is limited to monthly or shorter. The same set of control dosimeters was used for all irradiated dosimeter groups. The results are shown in Table 5.22. Because the variability of the control dosimeters is the dominant contributor to the calculated LLD in the equations provided in the test standard, similar values for LLD are expected for each source.

(a) J. Fix, “HSD (Wrist) Lower Level of Detection,” June 8, 1998, letter to HEDP file. 
Table 5.22 HSD Extremity Shallow Dose LLD

\begin{tabular}{||c|c|c||}
\hline Source & $\begin{array}{c}\text { Average } \\
\text { Energy } \\
(\mathrm{keV})\end{array}$ & $\begin{array}{c}\text { Shallow Dose } \\
\text { LLD } \\
(\mathrm{mrem})\end{array}$ \\
\hline${ }^{204} \mathrm{Tl}$ & $267^{*}$ & 3 \\
\hline${ }^{90} \mathrm{Sr} /{ }^{90} \mathrm{Y}$ & $931^{*}$ & 3 \\
\hline $\mathrm{M} 30$ & 20 & 3 \\
\hline $\mathrm{M} 60$ & 34 & 3 \\
\hline $\mathrm{M} 100$ & 51 & 3 \\
\hline $\mathrm{M} 150$ & 70 & 3 \\
\hline $\mathrm{H} 150$ & 117 & 3 \\
\hline${ }^{137} \mathrm{Cs}$ & 662 & 3 \\
\hline${ }^{60} \mathrm{Co}$ & 1252 & 3 \\
\hline
\end{tabular}

\subsubsection{Fading}

The fading properties of the HSD are not impacted by the body location on which the dosimeter is worn. Therefore, the fade characteristics described for the HSD Personnel dosimeter in Section 5.4.9 above are applicable for the HSD Extremity dosimeter as well. A single algorithm is used for the HSD whether used as an extremity or whole body badge. The fade corrections are applied in the algorithm on the basis of phosphor type, radiation type and number of days between anneal and readout.

\subsubsection{Environmental Sensitivity}

The description of environmental influences on the HSD as a personnel dosimeter given in Section 5.4.8 applies to the HSD when used as an extremity dosimeter as well. Precautions should be taken to avoid puncture of the beta window when the dosimeter is subject to contact with sharp objects.

\subsection{EXT-RAD Ring Dosimeter}

The primary dosimeter used for measuring extremity dose at Hanford is the EXTRAD ring dosimeter. This is a flexible plastic strap worn on the index finger of both hands. On a much less frequent basis, HSD wrist dosimeters are worn on wrists or ankles as extremity dosimeters (see description above).

\subsubsection{Dosimeter Description}

The EXT-RAD ring dosimeter contains a single ${ }^{7} \mathrm{LiF}: \mathrm{Mg}, \mathrm{Ti}$ (TLD-700) chip mounted on a thin polyamide $\left(\right.$ Kapton $\left.^{\circledR}\right)$ substrate with a permanent 5-digit barcode chip ID number. The Kapton ${ }^{\circledR}$ and chip assembly is commercially available from Harshaw under the product name Chipstrate ${ }^{\circledR}$, and is shown in Figure 5.28. The Harshaw product code for the specific chipstrate used by HEDP 
is "XD740." The chip consists of an active layer of TLD-700 phosphor in hotpressed chip form, adhesively bonded to a dosimetrically inert ${ }^{7} \mathrm{LiF}$ base. The density thickness of the active layer is approximately $40 \mathrm{mg} / \mathrm{cm}^{2}$. The dimensions of the TLD-700 chip are $3.2 \mathrm{~mm} \times 3.2 \mathrm{~mm} \times 0.15 \mathrm{~mm}$ and the dimensions of the inert base are approximately the same. The laminated chip is adhesively bonded to the Kapton ${ }^{\circledR}$ substrate. The permanent chipstrate ID number allows application of a chip-specific sensitivity factor to the dose result.

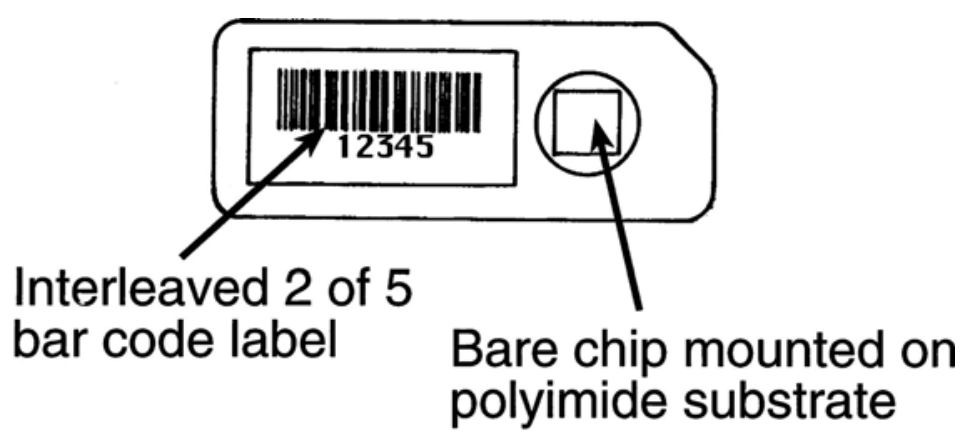

G00040106.2

Figure 5.28 Chipstrate

The EXT-RAD extremity dosimeter is prepared with the chipstrate sealed inside a vinyl pouch as shown in Figure 5.29. This pouch has an integral beta window with a density thickness of $7 \mathrm{mg} / \mathrm{cm}^{2}$.

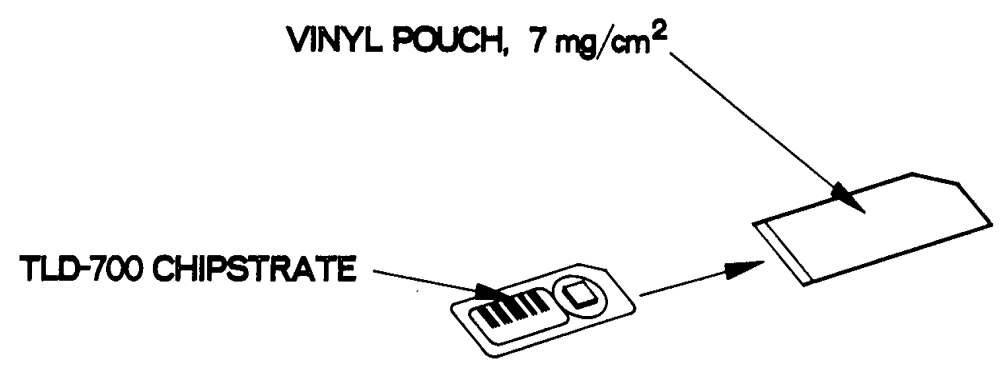

Figure 5.29 Illustration of Chipstrate Sealed in Vinyl Pouch

The vinyl pouch is inserted into a flexible black plastic holder with straps and a buckle that allow adjustment to individual finger size. The chipstrate ID number is visible through a window in the strap. An additional bar code label is placed on the back side of the vinyl pouch. This is a 5 digit sequential ring number which serves the following purpose: Rings are laced onto ring clips in numeric order at the time of preparation so that they can be assigned and issued to individuals in sequentially numbered pairs. Hanford practice is to wear odd-numbered rings on the left hand and even numbered rings on the right hand. An illustration of how the EXT-RAD is assembled is shown in Figure 5.30. 


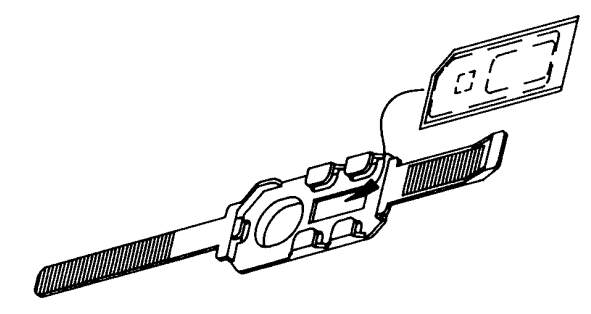

Figure 5.30 EXT-RAD Extremity Dosimeter

\subsubsection{Reader Description}

Chipstrates are read on one of two Harshaw Model 6600E hot gas readers. This reader contains two PMTs and two hot-gas heating jets. Prior to readout, chipstrates are inserted in aluminum carrier cards, two per card, which are then stacked in reader cartridges similar to those used on the Harshaw 8800 TLD readers. Hot nitrogen is used to heat the TLD chips according to a user-specified TTP. Nitrogen temperature is controlled to within $\pm 2^{\circ} \mathrm{C}$ of the programmed value. The nitrogen gas stream is directed at the underside of the chipstrate and heat transfer occurs through the Kapton ${ }^{\circledR}$ substrate. The use of nitrogen provides an inert atmosphere in the readout chamber, which reduces spurious readings caused by chemoluminescence of contaminants on the chip. Nitrogen also provides optimal heat transfer and uniform heating via a "non-contact" method of heating which allows for repeated readout of the chipstrate without the wear and deformation common to contact methods of heat transfer.

The Harshaw 6600E reader sensitivity for the XD740 chipstrate, using the standard TTP described below, is typically $0.060 \mathrm{nC} / \mathrm{mR}$. The gain on both $6600 \mathrm{E}$ readers has been adjusted to achieve a sensitivity in this range for each PMT. Average PMT dark current readings are less than $0.060 \mathrm{nC}$ which is the equivalent of a 1-mR reading for the XD740 chipstrate.

\subsubsection{Dosimeter Use Cycle}

The following sections briefly describe the processes involved in the ring dosimeter use cycle from preparation to dose reporting.

\subsubsection{Dosimeter Preparation}

Chipstrates are annealed in the $6600 \mathrm{E}$ reader using a TTP that starts at $50^{\circ} \mathrm{C}$ and ramps to $300^{\circ} \mathrm{C}$ at the rate of $25^{\circ} \mathrm{C} / \mathrm{sec}$ then holds at $300^{\circ} \mathrm{C}$ for six seconds. Chipstrates with an initial reading exceeding $100 \mathrm{mR}$ are re-read by the reader to ensure that residual signal is as low as possible. After reader annealing, the chipstrates (in carrier cards and reader cartridges) are oven annealed at $80^{\circ} \mathrm{C}$ for 16 hours. The reader annealing clears the primary longer-lived dosimetric traps, 
while the $80^{\circ} \mathrm{C}$ oven annealing reduces the number of short half-life traps, thereby reducing the fade of the overall TL signal.

After oven annealing, chipstrates are removed from the carrier cards, inserted into vinyl pouches, heat sealed, then inserted into the plastic strap. Sequential ring ID labels are placed on the back side of the dosimeter and the dosimeter is placed in sequential order on a ring clip for scanning and shipping to distribution centers.

\subsubsection{Dosimeter Issuance}

After assembly of the EXT-RADs is completed, the bar codes on the front (chipstrate ID) and the back (Ring ID) are scanned and the information stored in the External Dosimetry (ED) database. The chipstrate and ring number pairing, the date and time of scanning, contractor code, and other information are stored in a dosimeter TRACKING file in the same manner as for personnel dosimeters. Before a ring can be successfully scanned out to a contractor, the SCAN program checks the ED database to verify that the chipstrate has successfully passed all required acceptance tests, that it has been annealed within the past 30 days, and that it is available for issuance (i.e., not listed as damaged, in the process of being calibrated, or currently assigned). The TRACKING file is used to generate an ISSUE file which is used to update the REX database to make the dosimeters available for assignment to individuals.

\subsubsection{Dosimeter Receipt}

When the worker has finished wearing the dosimeters, they are physically returned to HEDP by the customer along with an electronic RETURN file of wearer/assignment information generated by REX. In addition to wear dates, wearer ID number, processing note code, and other pertinent information, this file also contains a facility calibration code. This code contains information about the correction factor that should be applied to the ring dose result. The dosimeters are surveyed for contamination, then scanned by HEDP staff to update the dosimeter TRACKING file showing the dosimeters as have been received. The scanning programs check to see if the dosimeter is returned with the same chipstrate ID number/ring ID number pairing as when issued as well as the dosimeter's last status in ED (i.e., last status = issued). This is a general feature of the SCAN code implemented primarily for use with personnel dosimeters.

\subsubsection{Dosimeter Readout}

After being successfully scanned in, each dosimeter is opened and the chipstrate is removed and cleaned as necessary. The chipstrates are then inserted in carrier cards, two per card, which are loaded into reader cartridges along with carrier cards containing QC and blank chipstrates. These chipstrates are then read as a group on one of the 6600 readers using a TTP specified for dosimetric readout. As the chipstrates are read, the TLD reader applies an RCF and an ECC to each chipstrate to obtain a reading in $\mathrm{mR}$. Chipstrates for which no ECC is available, will be ejected to the reject bin without being read, and a message as to why this occurred is placed in the reader log. Likewise, the reader will not read chipstrates 
with an uncalibrated TTP (a TTP for which no RCF has been determined). While processing chipstrates, the reader applies real-time process QC in the form of upper and lower limits on PMT noise readings, reference light readings, QC chipstrate readings, and blank chipstrate readings. Any reading that exceeds a programmed limit will cause the reader to stop reading. Process QC limits for QC chipstrates are $\pm 10 \%$ of the given exposure value (i.e., $450 \mathrm{mR}$ to $550 \mathrm{mR}$ ).

\subsubsection{Dose Calculation}

After being processed by the reader, the chipstrate readings are transferred to the External Dosimetry Data Management System where wearer/assignment information from the RETURN file provided by REX is matched with the individual ring ID number/chipstrate ID number, and doses calculated. The facility calibration code provided in the RETURN file is used by the dose calculation algorithm to calculate dose. The quantity calculated and reported to REX is shallow personal dose equivalent, $\mathrm{H}_{\mathrm{p}}(0.07)$, in units of mrem.

\subsubsection{Dose Reporting}

After wearer information is matched with the dosimeter and dose results have been calculated, any QC-related flags placed on the dose record must be investigated and cleared, and doses recalculated as necessary. When a dose record is eligible for reporting, a dose results file (REXDOSE) is generated. For Hanford users, the file is transmitted to REX where the results are incorporated into the REX database. For each pair of rings assigned to an individual, REX chooses the higher of the two ring results to use as the "ring" dose for the purpose of calculating equivalent dose to the extremities (see Chapter 4, Table 4.1). Dose results for extremity dosimeters are reported on a daily basis when available, as are results for personnel dosimeters.

\subsubsection{System Calibration}

The following sections briefly describe the methods used to calibrate various elements of the extremity dosimetry system. These methods are very similar to those used for the personnel dosimetry system.

\subsubsection{Reader Calibration}

The Harshaw Model 6600 TLD reader is calibrated by reading calibration cards that have been exposed to $500 \mathrm{mR}$ with a ${ }^{60} \mathrm{Co}$ source located in the 318 calibration facility's high-exposure room. The calibration cards consist of chipstrates which have been calibrated for individual chip sensitivity and permanently loaded into carrier cards. Annealed calibration cards are placed on a Plexiglas ${ }^{\mathrm{TM}}$ rack approximately $1 / 8$ inch thick, at a distance of approximately 7 meters from the source. Exposure rates at this distance have been established using NIST-traceable ionization chambers and electrometers, and are re-evaluated annually. The calibration cards are read with ECCs applied, using a defined TTP, and the reader calibration program run to establish an RCF for that TTP, 
expressed in $\mathrm{nC} / \mathrm{mR}$. This RCF can then be applied on a real-time basis to all chipstrates subsequently read using the given TTP. The reader calibration process and RCF calculation methodology are the same as described for personnel dosimeters in Section 5.3.2 and Section 5.3.4. Reader calibrations are performed weekly using a set of calibration cards that are prepared on a monthly basis and may be used up to 45 days after irradiation. Fade and background effects attributed to the 45-day use window are relatively small (less than $5 \%$ and $7 \mathrm{mR}$ respectively).

\subsubsection{Chipstrate Calibration}

Individual chipstrates are calibrated by exposing them to $500 \mathrm{mR}$ from a ${ }^{60} \mathrm{Co}$ source, reading them on a calibrated reader, and comparing the individual chip response with the mean response of the chipstrate population (estimated by the mean response of the sample of calibration cards used to calibrate the reader). The resulting chip sensitivity correction factor is referred to as an ECC, and is applied by the reader on a real time basis to all subsequent readings of the chipstrate. When the ECC is applied to raw chipstrate readings, a uniform response among chipstrates is achieved, with a standard deviation typically less than $3 \%$ for a $500 \mathrm{mR}$ exposure. The ECC calculation methodology used for chipstrates is the same as that described in Section 5.3.3.

\subsubsection{Calibrated Chip Readings}

When field, QC or blank chipstrates are processed for measurement of dose or process QC, the reader software applies the ECC and RCF to the raw chip reading to obtain a "calibrated chip reading", $\mathrm{X}$, in ${ }^{60} \mathrm{Co}$ mR-equivalent as follows:

$$
X=Q * E C C / R C F
$$

$$
\begin{aligned}
\text { where } \mathrm{X} & =\text { calibrated chip reading }(\mathrm{mR}) \\
\mathrm{Q} & =\text { PMT charge collected }(\mathrm{nC}) \\
\text { ECC } & =\text { element correction coefficient } \\
\text { RCF } & =\text { reader calibration factor }(\mathrm{nC} / \mathrm{mR}) .
\end{aligned}
$$

The file of chipstrate readings created by the reader (Group File) includes the calibrated chip reading, the ECC applied, and the RCF applied.

\subsubsection{Ring Dosimeter Calibration}

Dosimeter calibration consists of determining the relationship between a chip's response to the local source and geometry ( ${ }^{60} \mathrm{Co}$ in air outside holder), and its response to the calibration standard $\left({ }^{137} \mathrm{Cs}\right.$ in ring holder, on-phantom). The resulting factor is called the ${ }^{137} \mathrm{Cs} \mathrm{RRF}$ and is expressed in units of $\mathrm{mR} / \mathrm{mrem}$. The RRF is a function of the local and standard sources used, the chip thickness, and the filtration over the chip when in the holder. Therefore, the RRF varies with chip position and dosimeter type for the personnel dosimetry system. For the extremity dosimetry system, there is a single chip position and dosimeter type at present. The RRF is determined by exposing a set of chipstrates to the local 
source and a set of chipstrates to the calibration standard and reading them interleaved together in a single group on a stable reader with ECCs applied. The ratio of the average response to the calibration standard (nC/mrem) to the average response to the local source $(\mathrm{nC} / \mathrm{mR})$ is calculated. This ratio is the $\mathrm{RRF}$ for the EXT-RAD ring dosimeter. Dividing the calibrated reading $(\mathrm{mR})$ for a given chipstrate by the RRF for the ring dosimeter, provides a ${ }^{137} \mathrm{Cs}$ mrem-equivalent reading for that chip. This is the same reading that would have been obtained if the reader had been calibrated directly with chipstrates exposed in-holder onphantom to the calibration standard. The use of local sources in conjunction with RRFs results in more cost effective and reproducible calibration of the TLD system. The phantom, geometry, and source used as the calibration standard are as specified in HPS N13.32 - 1995 (HPS 1996a). [As of this writing, DOELAP has not implemented HPS N13.32 - 2008 (HPS 2008) for performance testing.]

\subsubsection{Dose Algorithm for EXT-RAD Ring Dosimeter}

Because the EXT-RAD ring dosimeter currently in use at Hanford is a single element dosimeter, the dose calculation algorithm is relatively simple compared with the algorithms used for the four-element HSD and HCND dosimeters. The following formulae describe the dose calculation methodology in sufficient detail to allow calculation by hand for verification of results if necessary.

\subsubsection{Shallow Personal Dose Equivalent}

$$
H_{p}(07)=\quad D * C F
$$

where:

$$
\begin{array}{lll}
\mathrm{H}_{\mathrm{p}}(07)= & \text { shallow personal dose equivalent (mrem) } \\
\mathrm{D} & = & \text { adjusted reading (mrem) } \\
\mathrm{CF} & = & \text { facility correction factor (dimensionless) }
\end{array}
$$

\subsubsection{Facility Calibration Factor}

A facility-specific calibration factor, CF, is determined from the two-digit facility calibration code, FCC, provided by the field dosimetry organizations in the RETURN file from REX as follows:

$\begin{array}{lll}\text { If } & \text { FCC }= & 00 \\ \text { Then } & \text { CF }= & 2.0 \\ \text { Else } & \text { CF }= & \text { FCC } / 10 \\ & & \end{array}$

\subsubsection{Adjusted Element Reading}


The method of calculating adjusted element readings for chipstrates is essentially the same as the one used for the 8816 and 8825 TLD cards that are used in the HCNDs and HSDs. Adjusted readings for chipstrates are calculated as follows:

$$
\begin{array}{ll} 
& D \quad=(X-E) /\left(R R F * F^{*} S\right) \\
\text { where: } \mathrm{D} \quad \text { = adjusted chip reading }\left({ }^{137} \mathrm{Cs} \text { mrem equivalent }\right) \\
\mathrm{X}=\text { calibrated chip reading }\left({ }^{60} \mathrm{Co} \mathrm{mR} \text { equivalent }\right) \\
\mathrm{E}=\text { estimated environmental background for chipstrate }\left({ }^{60} \mathrm{Co} \mathrm{mR}\right. \\
\quad \text { equivalent }) \\
\mathrm{RRF}={ }^{137} \mathrm{Cs} \text { RRF for ring }(\mathrm{mR} / \mathrm{mrem}) \\
\mathrm{F} \quad=\text { fade correction factor for chipstrate } \\
\mathrm{S} \quad=\text { supralinearity correction factor. }
\end{array}
$$

The empirically determined ${ }^{137} \mathrm{Cs}$ RRF for the EXT-RAD is $1.037 \mathrm{mR} / \mathrm{mrem}^{\text {(a) }}$

\subsubsection{Zero Dose Reading}

The zero dose reading for unexposed chipstrates averages approximately $10 \mathrm{mR}$ and this value is used as a constant (B) in Equation 5.13. The zero dose reading was determined by annealing 100 relatively new chipstrates and reading them on a calibrated reader two days later. This value accounts for both reader background and intrinsic chip background.

\subsubsection{Environmental Background Function}

The variable E, represents the portion of the chipstrate reading accumulated during the field cycle that is due to natural background radiation plus typical reader background and intrinsic chip background. E is calculated from an empirically determined background function based on the number of days in the field. For chipstrates, E is calculated as follows:

$\begin{array}{rll}E & =\quad G * F D+B \\ \text { where: } \mathrm{G} & = & \text { background growth rate }(0.145 \mathrm{mR} / \mathrm{d}) \\ \mathrm{FD} & = & \text { field days (days between previous and current } \\ \text { processing date) }\end{array}$

\subsubsection{Fade Correction}

Fade corrections for the chipstrate are based on an empirically determined two compartment exponential post-irradiation fade model for TLD 700 as used at Hanford. $^{(b)}$ Although the model is based on fading in TLD 700 chips in 8825

(a) S. E. Huneycutt, "Relative Response Factors for Rings,” August 30, 2001, letter to HEDP file.

(b) B. A. Rathbone, "Re-evaluation of Post Irradiation Fading of Beta-Gamma Dose in TLD 600 and TLD 700," March 20, 2000, letter to HEDP file. 
cards, it is considered valid for chipstrates as well because the same annealing and readout techniques are used for chipstrates. The model is as follows:

$$
F(t)=R(t) / R_{0}=a e^{\left(-\lambda_{1} t\right)}+(1-a) e^{\left(-\lambda_{2} t\right)}
$$

where: $\quad t$ is the time since irradiation (days)

$\mathrm{R}(\mathrm{t})$ is the net chip response $(\mathrm{mR})$ at time $\mathrm{t}$

$\mathrm{R}_{0}$ is the net chip response $(\mathrm{mR})$ at time $\mathrm{t}=0$

a is the weighting factor for the short half-life compartment

$\lambda_{1}$ is the decay constant for the short half-life compartment

$\lambda_{2}$ is the decay constant for the long half-life compartment

For routine dosimetry, when the time since irradiation is generally not known, one half of the time between previous and current processing date is used for $t$. The parameters to be used in the model are shown in Table 5.23. The model is shown graphically in Figure 5.31.

Table 5.23 Parameters for use in Chipstrate Post Irradiation Fade Model

\begin{tabular}{||l|l||}
\hline Parameter & TLD 700 $\beta-\gamma$ \\
\hline $\mathrm{a}$ & 0.0530 \\
\hline$\lambda_{1}$ & $0.0179 \mathrm{~d}^{-1}$ \\
\hline$\lambda_{2}$ & $0.000231 \mathrm{~d}^{-1}$ \\
\hline
\end{tabular}

\section{Post Irradiation Fading of Beta-Gamma Signal in TLD 700}

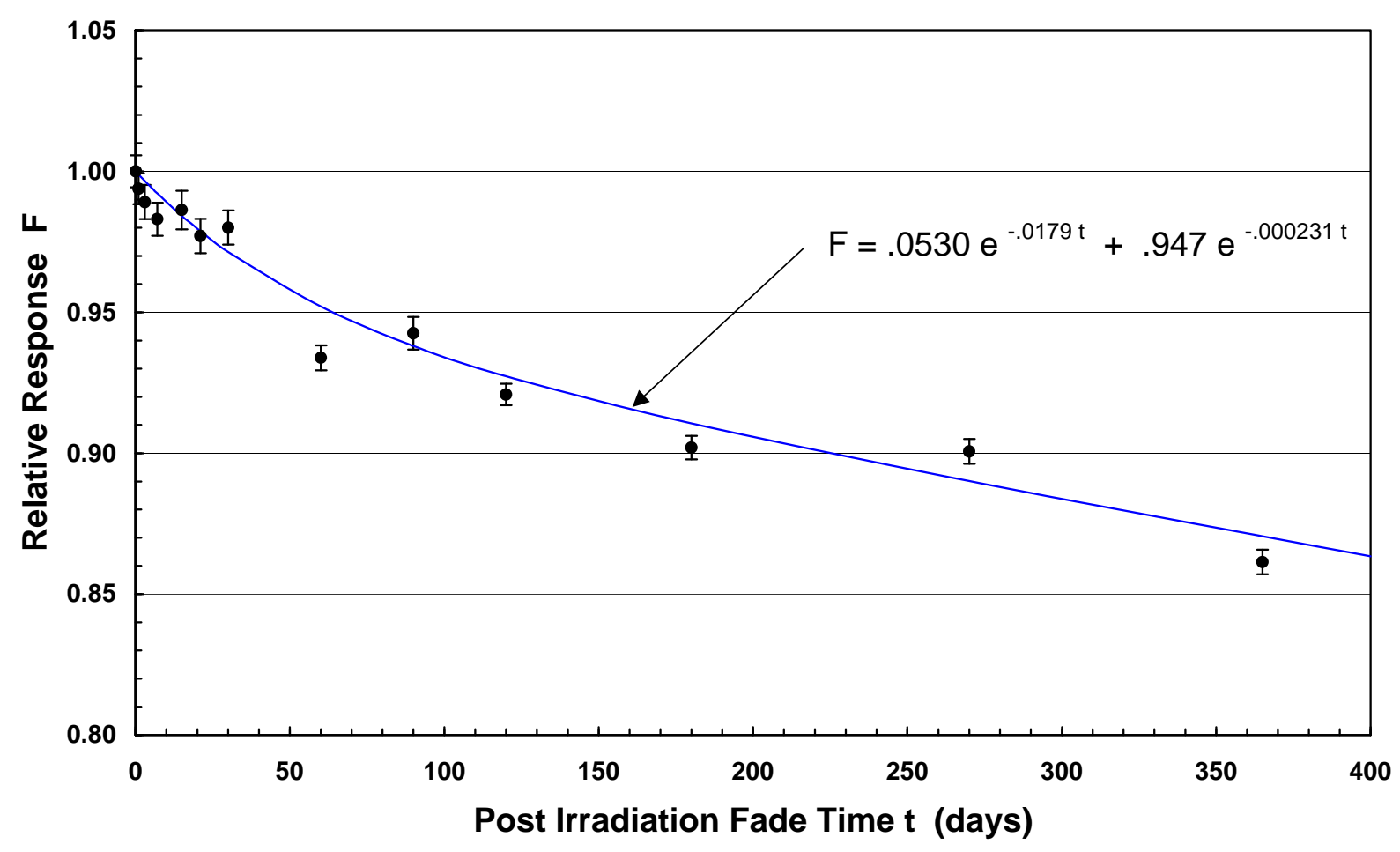

Figure 5.31 Fade Correction for the Hanford Ring 


\subsubsection{Supralinearity Correction}

Supralinearity correction for the chipstrate is taken to be the same as for other TLD-700 chips because supralinearity is a function of the phosphor type, radiation type, and annealing/readout protocol in addition to absorbed dose. Based on the supralinearity correction established for TLD 700 in personnel dosimeters the supralinearity correction used for chipstrates is as follows:

$$
S=\left(1.0+3.411 * 10^{-7} * \text { Xnet }\right)
$$

where:

$$
\text { Xnet }=\mathrm{X}-\mathrm{E}=\text { background corrected chip reading }(\mathrm{mR})
$$

\subsubsection{Performance Data}

The following sections briefly describe the basic performance characteristics of the EXT-RAD ring dosimeter. The results of formal DOELAP and NVLAP performance testing are available in HEDP files. [HEDP no longer maintains NVLAP accreditation for the EXT-RAD.]

\subsubsection{Uniformity}

Uniformity of response is defined as the coefficient of variation of response for many dosimeters given the same dose. Ideally, a measurement of uniformity of response should be conducted in a manner that controls for influences such as environmental background, fading, reader background and sensitivity, such that they do not contribute significantly to the measured variability of response.

Uniformity of response for the XD740 chipstrate without holder was determined by exposing 20 chipstrates in carrier cards to a ${ }^{60} \mathrm{Co}$ source in air at a distance of 7.14 meters. The $\% \mathrm{C}$.V. of the 20 calibrated chip readings was $1.5 \%$.

Uniformity of response for assembled dosimeters was determined by irradiating 10 dosimeters on a Plexiglas ${ }^{\mathrm{TM}}$ extremity rod phantom to 1000 mrem shallow personal dose equivalent of ${ }^{137} \mathrm{Cs}$ radiation and reading the dosimeters after 30 days of fade. The \% C.V. of the 10 reported doses was $2.5 \%$. The characteristic $\%$ C.V. in response for blind audit dosimeters exposed to ${ }^{137} \mathrm{Cs}$ is approximately $5 \%$. These are dosimeters that are prepared from random inventory of dosimetry materials on different dates over the course of a year, exposed on different dates, to different doses, and processed on different dates, such that fading, background and reader sensitivity are all variable in a manner representative of routine dosimetry.

\subsubsection{Lower Limit of Detection}

Initial determination of the LLDs for the EXT-RAD ring dosimeter was made using the method described in HPS N13.32 (HPS 1996a), Performance Testing of Extremity Dosimeters. The calculations were based on dosimeters assembled from the general population of chipstrates used for personnel dosimetry. Five sets of 30 dosimeters were exposed to five sources corresponding to the sources listed in HPS N13.32 (M30, M100, ${ }^{137} \mathrm{Cs},{ }^{90} \mathrm{Sr} /{ }^{90} \mathrm{Y}$, and ${ }^{204} \mathrm{Tl}$ ). A sixth set of 36 
dosimeters was left unexposed. Only 30 of the 36 unirradiated dosimeters were used for the LLD calculations in order to match the size of the exposed sets. The dosimeters were processed 85 days after they were annealed as a single group. The results of the LLD calculations for these sources can be seen in Table 5.24. To allow determination of the LLDs for the M60 and M150 beam codes shown in Table 5.24, a second LLD calculation was performed using the alternate method described in HPS N13.32 (HPS 1996a). This calculation used the results of all 36 of the unirradiated dosimeters discussed earlier along with the results of the NVLAP performance tests (B and S) from the test session completed in January 2002. The calculated LLDs for all sources are shown in Table 5.24. The raw data and calculations are documented in greater detail in HEDP files ${ }^{(a)}$.

The calculated LLDs from this "laboratory" study may not be reflective of the actual detection threshold achievable with routinely exchanged dosimeters for the following reasons: 1 ) exchanged dosimeters experience variable background dose rates, 2) exchanged dosimeters can experience rougher handling during use which may result in damage or contamination (non-radiological) of the chip (the chipstrates used for the study were new and recently calibrated).

Table 5.24 LLDs for EXT-RAD Ring Dosimeter

\begin{tabular}{||c|c|c||}
\hline \hline Source & Ring CF & LLD (mrem) \\
\hline M30 & 1.43 & 3.4 \\
\hline M60 & 1.59 & 3.0 \\
\hline M100 & 1.60 & 3.0 \\
\hline $\mathrm{M} 150$ & 1.45 & 3.3 \\
\hline${ }^{137} \mathrm{Cs}$ & 1.00 & 4.8 \\
\hline${ }^{90} \mathrm{Y}$ & 1.06 & 4.5 \\
\hline${ }^{204} \mathrm{Tl}$ & 0.84 & 5.5 \\
\hline
\end{tabular}

\subsubsection{Linearity}

Because linearity is a function of the TL phosphor and annealing/readout protocol, it is assumed that this characteristic for the personnel dosimeters and chipstrates will be essentially the same. Based on the response of Teflon ${ }^{\circledR}$ encapsulated TLD-700 chips using the same readout and annealing protocols as used for chipstrates, linearity for chipstrates is within $\pm 5 \%$ of the given dose from $10 \mathrm{mrad}$ to $100 \mathrm{rad}$. Above $100 \mathrm{rad}$, supralinearity corrections become significant. Supralinearity corrections are automatically applied to all ring readings using the relationship in Equation 5.15.

\subsubsection{Angular Response}

A study was conducted to measure the angular response of the EXT-RAD ring dosimeter to photons and beta particles of different energies. The study was performed using the sources and protocols described in HPS N13.32-1995

(a) S. E. Huneycutt, “LLD Calculations for EXT-RAD Ring Dosimeter,” July 3, 2002, letter to HEDP file. 
Performance Testing of Extremity Dosimeters (1996a). Two sources from category II (M30 and M100), one source from category III $\left({ }^{137} \mathrm{Cs}\right)$, and two sources from category IV $\left({ }^{90} \mathrm{Sr} /{ }^{90} \mathrm{Y}\right.$ and $\left.{ }^{204} \mathrm{Tl}\right)$ were used in this study. The delivered shallow dose for all sources was approximately 5 rem. Exposures were made on phantom at angles of $0^{\circ}, \pm 30^{\circ}, \pm 60^{\circ}, \pm 85^{\circ}$, and $180^{\circ}$ vertical orientation and $\pm 30^{\circ}, \pm 60^{\circ}$, and $\pm 85^{\circ}$ horizontal orientation. Rotation in the horizontal orientation is illustrated as Polar Rotation while rotation in the vertical orientation is illustrated as Azimuthal Rotation in Figure 5.34. Positive angles are in the clockwise (CW) direction. Negative angles are in the counter-clockwise direction (CCW). Irradiations with photon sources were made at a distance of 100 $\mathrm{cm}$ while irradiations with beta sources were made at $35 \mathrm{~cm}\left({ }^{90} \mathrm{Sr} /{ }^{90} \mathrm{Y}\right)$ and $30 \mathrm{~cm}$ $\left({ }^{204} \mathrm{Tl}\right)$. The ${ }^{204} \mathrm{Tl}$ exposures were conducted with a flattening filter in place whereas the ${ }^{90} \mathrm{Sr} /{ }^{90} \mathrm{Y}$ irradiations were not. All irradiations were conducted by the Battelle Calibration Research and Accreditation group using the sources, phantoms, geometry, and $C_{x}$ factors specified in HPS N13.32-1995 (1996a). The results are summarized in Table 5.25 and shown graphically in Figures 5.32 and 5.33. The raw data are included in HEDP files ${ }^{(a)}$.

For the photon sources in Table $\mathbf{5 . 2 5}$ the response data for horizontal orientations are based on three rings irradiated on phantom in one exposure and the data for vertical orientations are based on five rings irradiated on phantom in one exposure. For the beta sources, response data for horizontal orientations are based on four rings irradiated on phantom two at a time. The data for vertical orientations are based on three rings irradiated on phantom in one exposure. The angular response for each combination of source, angle and orientation, was calculated as the average reported/given value divided by the average reported/given value at zero degrees for the same source and orientation.

Table 5.25 EXT-RAD Ring Angular Shallow Dose Response

\begin{tabular}{||c|c|c|c|c|c|c|c|c|c|c||}
\hline \hline Source & $\begin{array}{c}\text { Average } \\
\text { Energy } \\
(\mathrm{keV})\end{array}$ & $\begin{array}{c}\text { Axis of } \\
\text { Rotation }\end{array}$ & $-85^{\circ}$ & $-60^{\circ}$ & $-30^{\circ}$ & $0^{\circ}$ & $30^{\circ}$ & $60^{\circ}$ & $85^{\circ}$ & $180^{\circ}$ \\
\hline $\mathrm{M} 30$ & 20 & $\mathrm{H}$ & 0.44 & 0.86 & 0.99 & - & 0.98 & 0.89 & 0.45 & - \\
\hline & & $\mathrm{V}$ & 0.50 & 0.86 & 0.97 & 1.00 & 0.97 & 0.87 & 0.69 & 0.17 \\
\hline $\mathrm{M} 100$ & 51 & $\mathrm{H}$ & 0.81 & 0.98 & 1.01 & - & 1.03 & 1.00 & 0.84 & - \\
\hline & & $\mathrm{V}$ & 0.86 & 0.96 & 0.99 & 1.00 & 1.00 & 0.96 & 0.91 & 0.60 \\
\hline${ }^{137} \mathrm{Cs}$ & 662 & $\mathrm{H}$ & 1.01 & 1.01 & 1.00 & - & 1.00 & 1.02 & 1.01 & - \\
\hline & & $\mathrm{V}$ & 0.97 & 0.99 & 0.99 & 1.00 & 1.00 & 0.99 & 0.99 & 0.91 \\
\hline${ }^{90} \mathrm{Sr}{ }^{90} \mathrm{Y}$ & 931 & $\mathrm{H}$ & 0.17 & 0.75 & 1.03 & - & 1.07 & 0.70 & 0.19 & - \\
\hline & & $\mathrm{V}$ & 0.17 & 0.72 & 1.05 & 1.00 & 1.03 & 0.85 & 0.24 & 0.004 \\
\hline${ }^{204} \mathrm{Tl}$ & 267 & $\mathrm{H}$ & 0.12 & 0.42 & 0.83 & - & 0.81 & 0.39 & 0.12 & - \\
\hline & & $\mathrm{V}$ & 0.13 & 0.44 & 0.85 & 1.00 & 0.87 & 0.43 & 0.17 & 0.006 \\
\hline
\end{tabular}

(a) S. E. Huneycutt, “EXTRAD Ring Dosimeter Angular Dependence,” July 3, 2002, letter to HEDP file. 


\section{Shallow Dose Angular Response}

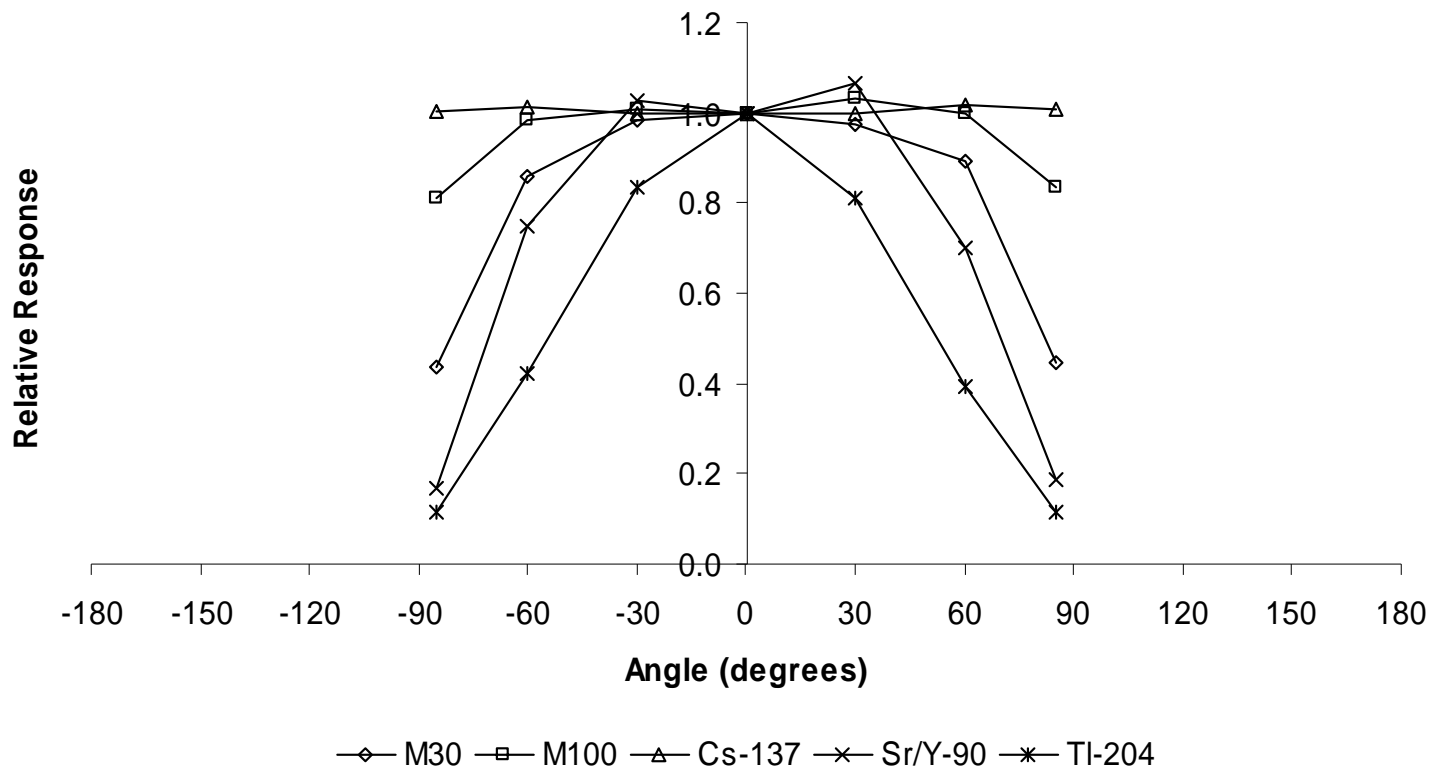

Figure 5.32 EXT-RAD Ring Shallow Dose Angular Response - Horizontal Orientation

\section{Shallow Dose Angular Response}

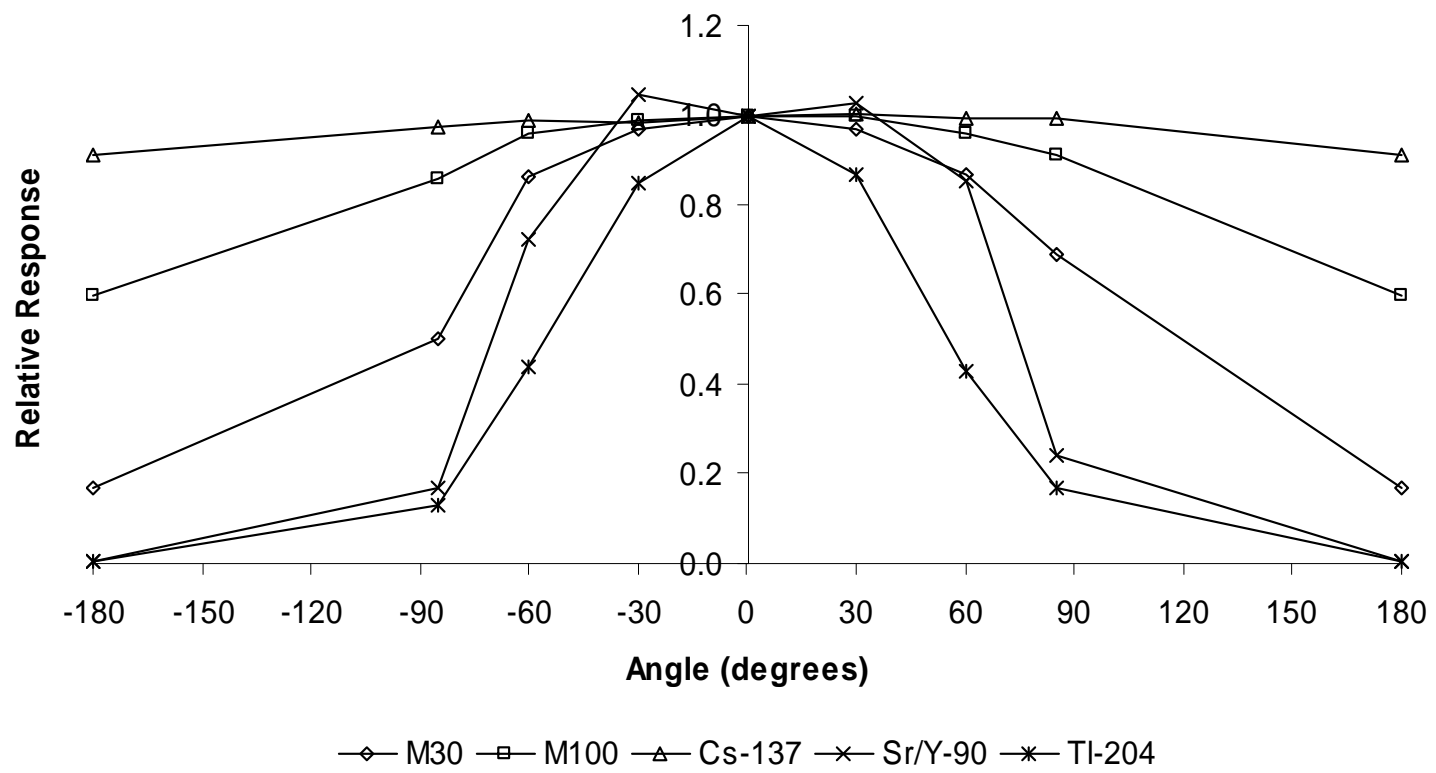

Figure 5.33 EXT-RAD Ring Shallow Dose Angular Response - Vertical Orientation 

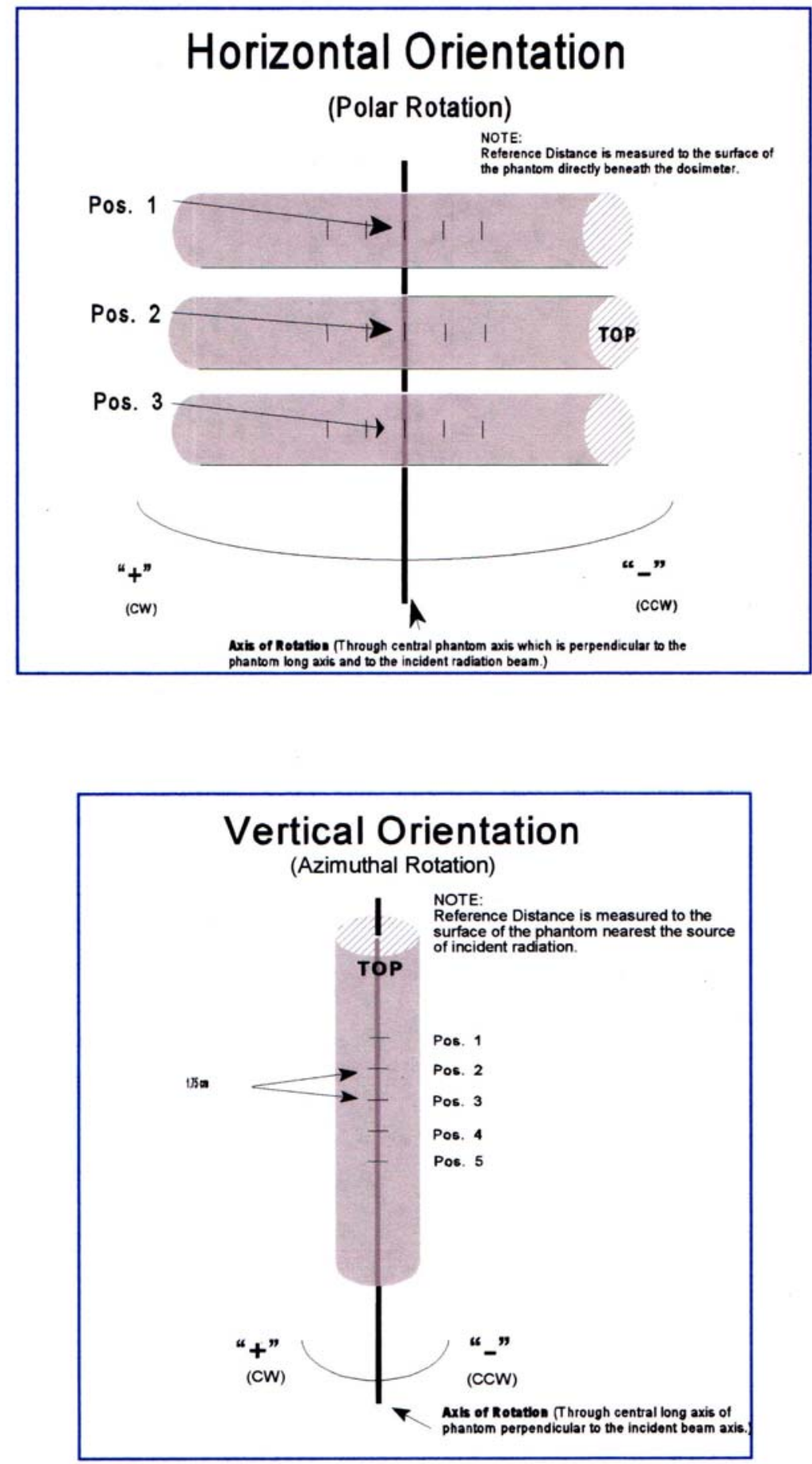

Figure 5.34 Ring Orientation for Angular Dependence Irradiations 


\subsubsection{Photon Energy Dependence}

Because there are no energy-flattening filters in the ring and no energy compensation is possible in the algorithm, the photon energy response of the EXT-RAD ring is typical of that for all ${ }^{7} \mathrm{LiF} \mathrm{TL}$ materials and follows closely the theoretical response curve based on the mass energy absorption coefficient for $\mathrm{LiF}$ as a function of photon energy. Photon energy response data were developed for the EXT-RAD ring by exposing dosimeters on-phantom to the beam codes and geometries specified in HPS N13.32-1995 (1996a). The $\mathrm{C}_{x}$ factors specified in this standard were used to derive the delivered shallow dose equivalent for each beam code. The photon response curve for the EXT-RAD ring dosimeter is shown in Figure 5.35.

\section{EXT-RAD Photon Response}

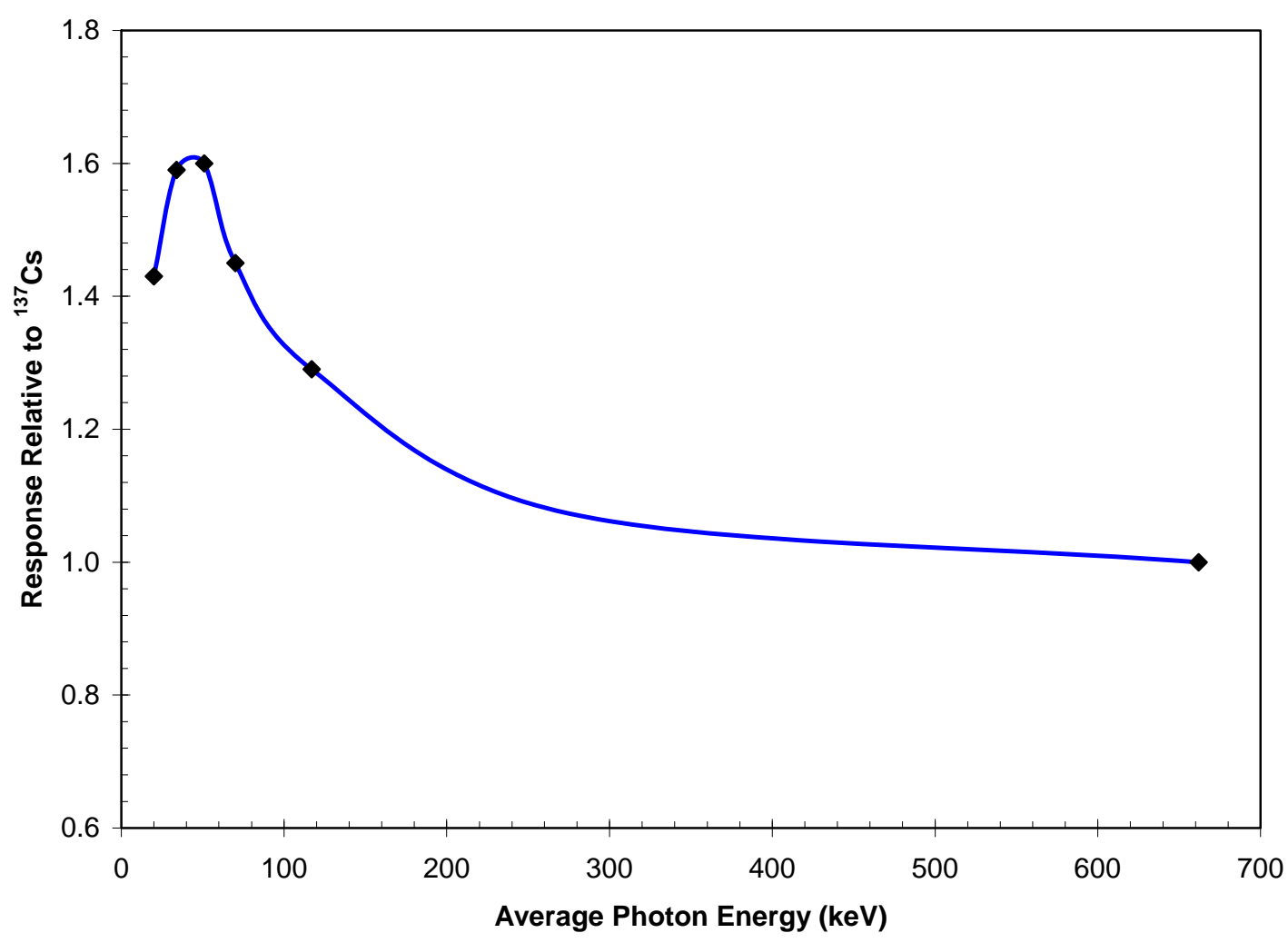

Figure 5.35 EXT-RAD Photon Response 


\subsubsection{Beta Energy Dependence}

Groups of five EXT-RAD ring dosimeters each were exposed to several rad from ${ }^{90} \mathrm{Sr} /{ }^{90} \mathrm{Y}$ and ${ }^{204} \mathrm{Tl}$ Buchler/PTB beta calibration standard sources. The ring response relative to the delivered shallow dose is shown in Table 5.26. The system is calibrated to ${ }^{137} \mathrm{Cs}$ photons.

Table 5.26 EXT-RAD Beta Response

\begin{tabular}{||l|l|l|l|l|}
\hline Source & $\beta_{\max }(\mathrm{keV})$ & $\beta_{\text {avg }}(\mathrm{keV})^{\mathrm{a}}$ & Reported/Given & BCF \\
\hline${ }^{90} \mathrm{Sr} /{ }^{90} \mathrm{Y}^{\mathrm{b}}$ & 2240 & 931 & 1.06 & 0.94 \\
\hline${ }^{204} \mathrm{Tl}$ & 765 & 267 & 0.84 & 1.19 \\
\hline
\end{tabular}

a. Nominal values. Actual energies are slightly less because of filtration inherent in encapsulation and beam flattener.

b. Most of the ${ }^{90} \mathrm{Sr}$ beta particles are removed from the beam by encapsulation and beam flattener.

\subsubsection{Default Correction Factor for the EXT-RAD Ring}

When the user enters a facility calibration code of 00 for the EXT-RAD ring in the REX database, the dose calculation algorithm applies a default correction factor of 2.0 to the uncorrected dose to obtain the final reported dose. The uncorrected dose is based on system calibration to ${ }^{137} \mathrm{Cs}$ gamma. This default correction factor is adequate for mixtures of ${ }^{90} \mathrm{Sr},{ }^{90} \mathrm{Y}$ and ${ }^{137} \mathrm{Cs}$ (the primary beta dose contributors at Hanford). For beta-emitters with average energies less than

${ }^{137} \mathrm{Cs}$, (approximately $200 \mathrm{keV}$ ) larger correction factors may be necessary. Although rare, exposure to such low energy beta emitters might be involved in laboratory work with special isotopes. If large quantities of a pure beta emitter are involved, it is possible for a worker wearing surgeon's gloves to receive measurable beta dose to the skin of the hands even when the average beta energies are less than $200 \mathrm{keV}$. If no photon dose is involved, it is conceivable that a correction factor larger than 2.0 might be required to accurately reflect the shallow personal dose equivalent received. If the quantities involved are large enough or the exposure is long enough, significant doses could be involved. It is the responsibility of the user to identify situations where correction factors other than the default are appropriate. The user should therefore characterize work environments and closely monitor work conditions to ensure that large beta/gamma dose ratios do not exist in conjunction with low-energy beta emitters and consult HEDP regarding appropriate correction factors when these conditions do exist.

The primary dose-contributing radionuclides presently encountered in many Hanford work environments are ${ }^{137} \mathrm{Cs}$ and ${ }^{90} \mathrm{Sr} /{ }^{90} \mathrm{Y}$ in secular equilibrium. These nuclides are most often encountered in tank waste characterization work and tank waste remediation work and often in association with large dose rates and dose gradients. Experience has shown large shallow doses to the extremity are most often associated with the handling of tank waste with large $\mathrm{Sr} / \mathrm{Cs}$ ratios in small containers. Typical containers are centrifuge cones and vials with wall thicknesses of approximately $100 \mathrm{mg} / \mathrm{cm}^{2}$, and $125-\mathrm{ml}$ glass sample jars with wall thicknesses of approximately $680 \mathrm{mg} / \mathrm{cm}^{2}$. Container walls thicker than 100 $\mathrm{mg} / \mathrm{cm}^{2}$ remove essentially all of the ${ }^{90} \mathrm{Sr}$ beta particles and begin to degrade the 
${ }^{90} \mathrm{Y}$ beta spectrum slightly. The default ring correction factor of 2.0 should be adequate for these situations.

Source terms that are primarily ${ }^{137} \mathrm{Cs}$ may also produce significant extremity dose rates when the activity is accumulated in $3 \mathrm{D}$ geometries such as pipes, valves, and sample containers (rather than a 2D surface geometry). These geometries tend to selectively shield low-energy beta radiation but not gamma radiation. In this situation, the predominant contributor to dose is photon radiation. The default ring correction factor of 2.0 should be adequate for these situations.

Based on the beta correction factors shown in Table 5.26, it would appear that a default ring correction factor equal to 2.0 is highly conservative. However, considerations such as potentially large dose gradients between maximally exposed areas of the hand (e.g. finger tips) and ring location, and potentially incorrect orientation of the ring with respect to the source, warrant the use of a 2.0 default correction factor.

\subsubsection{EXT-RAD Ring Correction Factor for the Plutonium Finishing Plant}

The EXT-RAD ring dosimeter has essentially no sensitivity to neutrons. Because of the need to account for unmeasured neutron dose to extremities which is incurred during glove box work with plutonium compounds at the Hanford PFP, a ring correction factor of 2.0 has been adopted for use in correcting ring results for PFP workers. The correction factor of 2.0 is based on neutron to gamma dose rate ratios between 1 and 2 assessed at PFP and correction factors used at other DOE sites for similar beta/gamma ring dosimeters used in handling plutonium. ${ }^{\text {(a) }}$ The factor takes into account the over-response of LiF to low-energy photons, which is about $150 \%$ of the true dose for the $60-\mathrm{keV}$ photons from ${ }^{241} \mathrm{Am}$ associated with aged plutonium. The factor also takes into account the fact that there is no beta radiation in PFP glove box operations to which the ring might under-respond. More recently, direct measurements inside leaded gloves used in PFP glove boxes produced average neutron to gamma ratios between 0.09 and 0.55 for a variety of plutonium oxide and metal sources in sealed cans (Scherpelz, Fix, and Rathbone 2000). In this study, for the purpose of calculating neutron to gamma ratios, the gamma reading from a TLD 700 chip in a Hanford ring was used as the gamma dose without correction for over response to low energy photons. Using a nominal neutron dose to gamma reading ratio of 0.5 obtained from this study, a correction factor of 1.17 on uncorrected ring results would be appropriate to account for neutron dose in the reported extremity dose. However, a variety of factors can have a great influence on the gamma fluence reaching the TLD 700 chip in the ring including; source dimensions (i.e. self shielding in the source), shielding in the cans, lead loading in the gloves used, orientation of the ring on the finger, age of source material, and others (DOE 1998b). For example, in the Scherpelz, Fix and Rathbone (2000) study, ring response was measured with and without gloves to determine the photon attenuation affect of the glove. The glove thickness was shown to be equivalent to almost a half value layer for some of the sources used. A simple addition of one half value layer from additional glove material, or lead shielding in the can, while having a negligible effect on the neutron dose rate would reduce the photon response of the ring by a factor of 2 . A

(a) J. J. Fix, “Extremity Dosimetry: Neutron to Photon Ratio,” August 4, 1997, letter to HEDP file. 
ring correction factor of 1.67 would then be necessary to correct for unmeasured neutron dose in the ring result. Given the uncertainties in photon shielding, the currently adopted ring correction factor of 2.0 provides appropriate conservatism. Because this factor is the same as the default ring correction factor for Hanford, a special facility calibration code is not required for rings worn at PFP (i.e. rings may be returned in REX with the default calibration code 00 ).

\subsection{Hanford Environmental Dosimeter}

The Hanford environmental dosimeter is intended for measurement of ambient radiation levels without phantom. The dosimeter holder is known commercially as a Harshaw 8807 dosimeter. The dosimeter contains 0.89 -mm-thick phosphors in all positions: TLD-200 in positions one and two, and TLD-700 in positions three and four. The dosimeter is illustrated in Figure 5.36.

\subsubsection{General Features}

Tawil et al. (1993) have shown that the 8807 environmental dosimeter meets all applicable requirements of ANSI N545 (ANSI 1975), as modified by the Nuclear Regulatory Commission (NRC's) Regulatory Guide 4.13 (NRC 1977). In particular the following performance was demonstrated:

Fade: less than $5 \%$ per quarter

Uniformity: less than $3 \%$

Reproducibility: less than $2.0 \%$

Light dependence: negligible

Moisture dependence: negligible

Self irradiation: negligible

Energy dependence: $\pm 20 \%$ from $20 \mathrm{keV}$ to $1.3 \mathrm{MeV}$. 


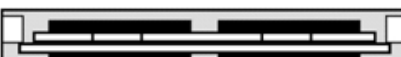

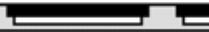

Note: Shielding Both Sides

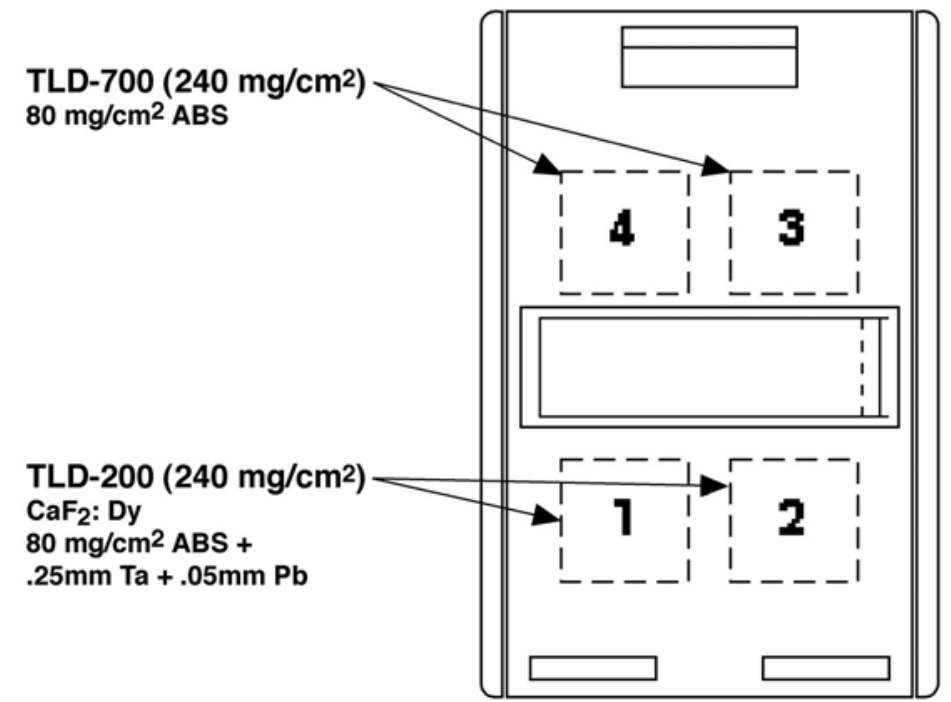

Hanford Environmental Dosimeter

39502026.2

Figure 5.36 Hanford Environmental Dosimeter (Harshaw 8807)

\subsubsection{Algorithm}

The 8807 algorithm has the following options: 1) use of the two TLD-700 chips for exposure calculation without energy correction, 2) use of the two TLD-700 chips for exposure calculation with energy correction based on the TLD200/TLD-700 chip ratio, or 3) exposure calculation based on all four chips with energy correction to all four based on the TLD-200/TLD-700 chip ratio. At Hanford, the exposure calculations are currently based on the first option because of the improved reproducibility of results that it offers.

\subsubsection{Processing Protocol}

The TTPs used for dosimetric readout (TTP 1) and annealing (TTP 2) of the Hanford environmental dosimeter 8807 card type are shown in Table 5.27. 
Table 5.27 Hanford Environmental Dosimeter Time-Temperature Profiles

\begin{tabular}{||l|r|r|r|l||}
\hline & \multicolumn{4}{|c|}{ TTP1(Field Reading) } \\
\hline Preheat temperature & 160 & 160 & 50 & $50^{\circ} \mathrm{C}$ \\
\hline Time & 25 & 25 & 0 & $0 \mathrm{sec}$ \\
\hline Temperature rate & 20 & 20 & 10 & $10^{\circ} \mathrm{C} / \mathrm{sec}$ \\
\hline Maximum & 300 & 300 & 300 & $300^{\circ} \mathrm{C}$ \\
\hline Acquire time & 20 & 20 & 33 & $33 \mathrm{sec}$ \\
\hline Annealing temperature & 300 & 300 & 300 & $300^{\circ} \mathrm{C}$ \\
\hline Time & 0 & 0 & 0 & $0 \mathrm{sec}$ \\
\hline & \multicolumn{5}{|c|}{ TTP2(Annealing) } \\
\hline Preheat temperature & 50 & 50 & 50 & $50^{\circ} \mathrm{C}$ \\
\hline Time & 0 & 0 & 0 & $0 \mathrm{sec}$ \\
\hline Temperature rate & 20 & 20 & 10 & $10^{\circ} \mathrm{C} / \mathrm{sec}$ \\
\hline Maximum & 300 & 300 & 300 & $300^{\circ} \mathrm{C}$ \\
\hline Acquire time & 40 & 40 & 33 & $33 \mathrm{sec}$ \\
\hline Annealing temperature & 300 & 300 & 300 & $300^{\circ} \mathrm{C}$ \\
\hline Time & 0 & 0 & 6 & $6 \mathrm{sec}$ \\
\hline
\end{tabular}

\subsubsection{Energy Response}

When run in the mode where energy correction of the TLD-700 readings is not used, the 8807 algorithm can be expected to report results within $30 \%$ of the true value. Data on energy response generated at the PNNL 318 Building Calibrations Facility based on the first option are shown in Figure 5.37.

\subsubsection{Lower Limit of Detection}

The lower limit of detection for the 8807 dosimeter was shown to be approximately 0.2 mrem for a monthly field cycle (Tawil et al. 1993).

\subsubsection{Fading}

Fading corrections for the 8807 dosimeter are based on the fade data generated for the TLD-700 phosphors used in HEDP personnel dosimeters. The anneal treatments are identical for TLD 700 phosphors used in personnel and environmental dosimeters. For a quarterly field cycle, the fade correction is about $5 \%$, depending upon the actual number of days between annealing and readout. 


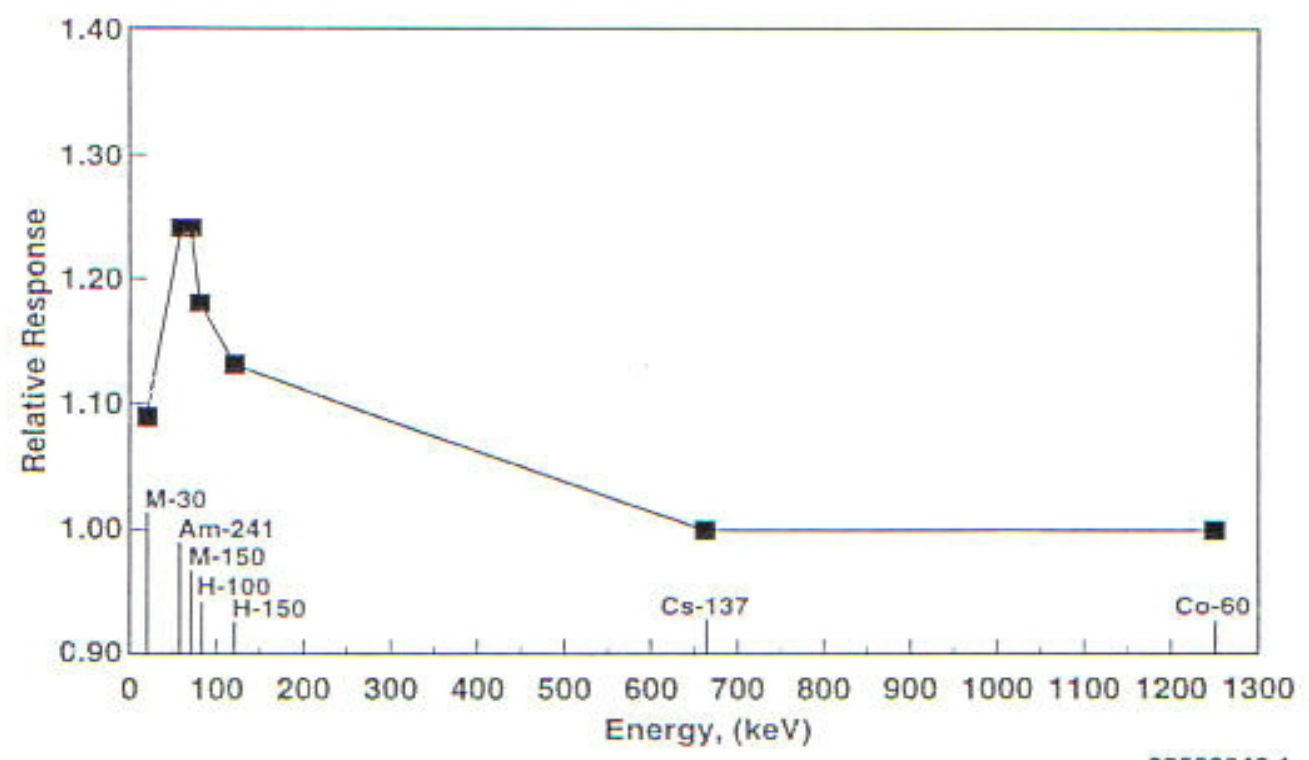

Figure 5.37 Hanford Environmental Dosimeter Energy Response (TLD 700)

\subsection{Hanford Nuclear Accident Dosimetry}

HEDP provides technical support to Hanford contractors for nuclear accident dosimetry involving four requirements in 10 CFR 835.1304 as follows:

1. a method to conduct initial screening of individuals involved in a nuclear accident to determine whether significant exposures to radiation occurred;

2. methods and equipment for analysis of biological materials;

3. a system of fixed nuclear accident dosimeter units (FNADs);

4. personal nuclear accident dosimeters (PNADs).

HEDP capabilities to support Hanford contractor compliance in nuclear accident dosimetry are described in this section. Hanford contractors are responsible for assignment of personnel dosimeters and PNADs, analysis of the placement of FNADs, documentation listing the location of each FNAD, and retrieval instructions for each affected facility. FNADs provide supplemental dosimetry information, which can be extrapolated to affected workers, in addition to dosimetry information available from the personnel dosimeter and PNAD assigned to the worker and dosimetry information available from biological samples and/or analyses of personal items (i.e., coins, rings, watches, etc.). 


\subsubsection{Hanford Nuclear Accident Dosimeters}

In order to provide as much dosimetry data as possible in a criticality event, both FNADs and PNADs are used at Hanford. Data from these dosimeters play an essential role in estimating dose in a criticality event.

Fixed Nuclear Accident

The Hanford FNAD has an outer and an inner dosimetry package as illustrated Dosimeter in Figure 5.38. The dosimetry materials in the FNAD are summarized in Table 5.28. The inner dosimetry package consists of a gold foil, a TLD 600 chip and a TLD 700 chip. When in place in the FNAD, the gold foil and TLD chips are positioned approximately at the geometrical center of the moderator. The outer dosimetry package consists of several neutron activation foils and a TLD 700 chip. These components are used collectively to provide the best estimate of the neutron and gamma dose resulting from a criticality. The neutron activation foils are used to estimate the neutron fluence in several energy ranges, as follows:

- thermal to $0.4 \mathrm{eV}$

- $0.4 \mathrm{eV}$ to $2 \mathrm{eV}$

- $0.4 \mathrm{eV}$ to $10 \mathrm{eV}$

- $2 \mathrm{eV}$ to $0.5 \mathrm{MeV}$

- above $1.2 \mathrm{MeV}$

- $\quad$ above $2.9 \mathrm{MeV}$.

General features of FNADs are presented in this section. Original design features of the Hanford FNAD are presented in reports by Bramson (1962) and by Glenn and Bramson (1977). The paraffin moderator of the Hanford FNAD is $30 \mathrm{~cm}$ high by $23 \mathrm{~cm}$ in diameter with 10 -cm-thick paraffin walls. The moderator is equipped with a $2.54 \mathrm{~cm}$ diameter polyethylene "candle" and polypropylene foil holder inserts.

Interpretation of dose is based on the method of calibration for each component, as well as the techniques used in the evaluation. The Hanford FNAD system has been tested several times over the years at the Oak Ridge National Laboratory (ORNL) Health Physics Research Reactor (HPRR) with good performance results. The results of the most recent test (conducted while the HPRR was still operating during August 1985) are maintained in the HEDP files. 


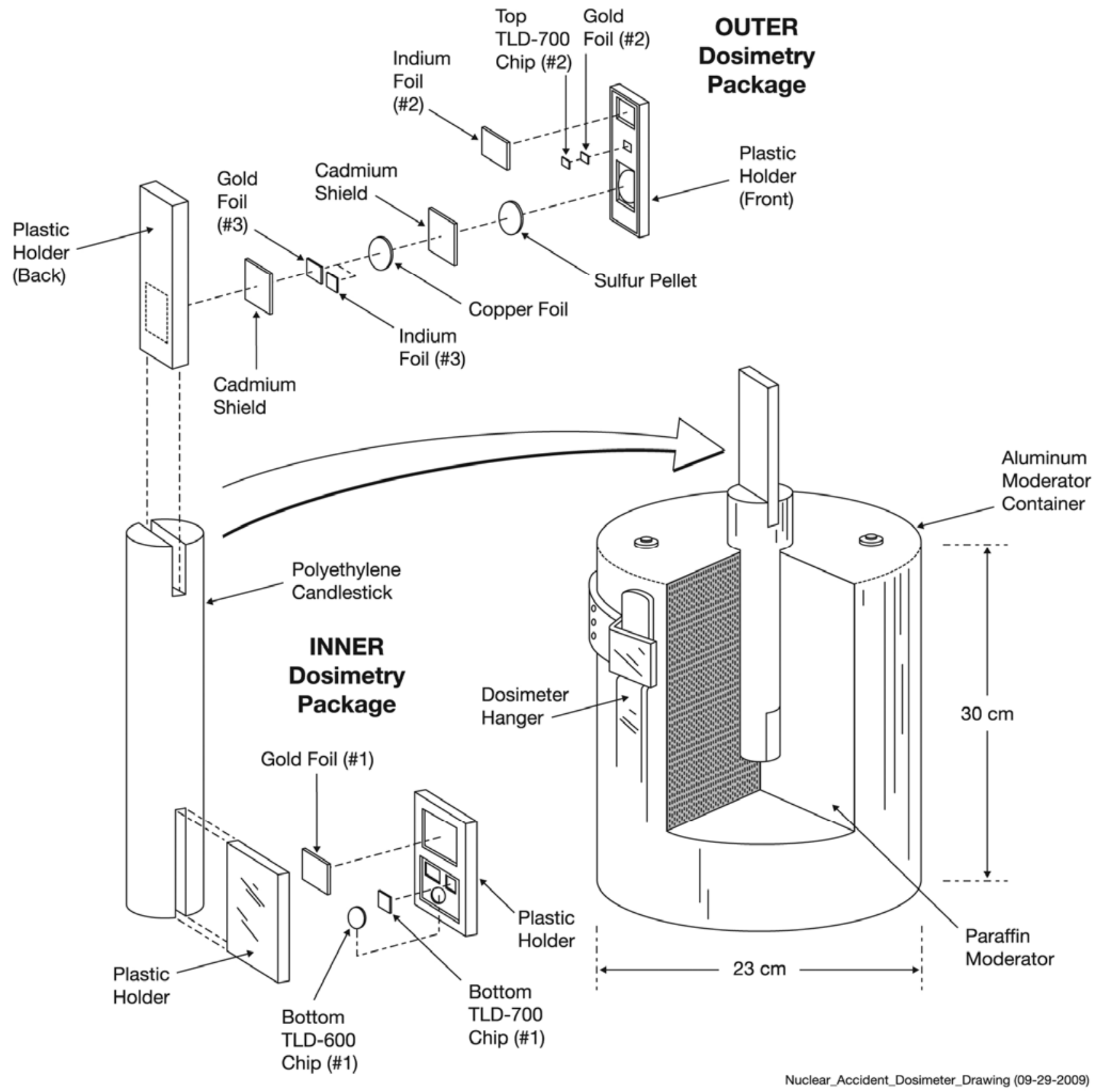

Figure 5.38 Hanford Fixed Nuclear Accident Dosimeter 
Table 5.28 Materials and Approximate Dimensions of FNAD Components

\begin{tabular}{|l|l|l|}
\hline \multicolumn{1}{|c|}{ Description } & \multicolumn{1}{|c|}{ Size, cm } & Thickness, cm (mil) \\
\hline Inner Dosimetry Package & & \\
Square gold foil (1) & & \\
TLD-700 chip (1) & 1.0 by 1.0 & $0.0127(5)$ \\
TLD-600 chip (1) & 0.32 by 0.32 & $0.089(35)$ \\
\hline Outer Dosimetry Package & 0.25 by 0.38 & $0.089(35)$ \\
\hline & & \\
Square gold foils (2, 3) & & \\
Indium foils (2, 3) & 1.0 by 1.0 & $0.0127(5)$ \\
Copper & 1.3 by 1.7 & $0.025(10)$ \\
Sulfur & 2.2 dia. & $0.025(10)$ \\
Cadmium shields & 1.27 dia. & $0.190(75)$ \\
TLD-700 chip (1) & 3.2 by 2.2 & $0.114(45)$ \\
\hline \hline
\end{tabular}

Personal Nuclear Accident Dosimeters
An illustration of the Hanford PNAD is shown in Figure 5.39. Table 5.29 lists the dosimetry components of the PNAD. The design of the Hanford PNAD is based on the outer dosimeter packet of the Hanford FNAD design and the PNAD used at the Los Alamos National Laboratory (LANL) (Vasilik and Martin 1981a). LANL tested their PNAD system at ORNL's HPRR Laboratory and documented these results (Vasilik and Martin 1981b). Performance of the Hanford PNAD is very similar to the performance observed with the outer dosimeter packet of the Hanford FNAD and the LANL documented data for the LANL PNAD. During 1997, a TLD-700 chip was added to the PNAD configuration to provide photon in addition to neutron radiation response characteristics.

The PNAD packets are issued by Hanford contractors to persons working in any area where a nuclear criticality event is possible. Each foil (i.e., including sulfur tablet) contained in the PNAD applies to a certain part of the energy spectrum. The total dose to which the PNAD was exposed is the sum of the individual spectrum-weighted dose components. The dose to the PNAD must be related to the dose to the person wearing the PNAD. The dose to a PNAD facing a criticality event will be different from the dose to a PNAD shielded by the body of the wearer. 
Table 5.29 Materials and Approximate Dimensions of PNAD Components

\begin{tabular}{|c|c|c|c|}
\hline Position & Description & Diameter, $\mathrm{cm}^{(\mathrm{c})}$ & Thickness, cm (mil) ${ }^{(\mathrm{c})}$ \\
\hline $\begin{array}{l}1 \\
2 \\
3 \\
4\end{array}$ & $\begin{array}{l}\text { Indium (Cd cover) } \\
\text { Sulfur (bare) } \\
\text { Indium (bare) } \\
\text { Copper (Cd cover) } \\
\text { TLD-700 chip }\end{array}$ & $\begin{array}{c}1.27 \\
1.27 \\
1.14 \\
1.27 \\
0.32 \times 0.32^{(b)}\end{array}$ & $\begin{array}{l}0.0127(5) \\
0.292(115) \\
0.0127(5) \\
0.0127(5) \\
0.089(35)\end{array}$ \\
\hline
\end{tabular}

(a) The cadmium enclosure, which contains indium and copper foils, is $0.051 \mathrm{~cm}$ (21 mil) thick.

(b) TLD-700 chip measures 0.32 by $0.32 \mathrm{~cm}(1 / 8$ by $1 / 8$ inch) square by $0.089 \mathrm{~cm}(0.035$ inch) in thickness. The TLD chip is located between the bare indium foil and sulfur pellet.

(c) All dimensions are nominal.

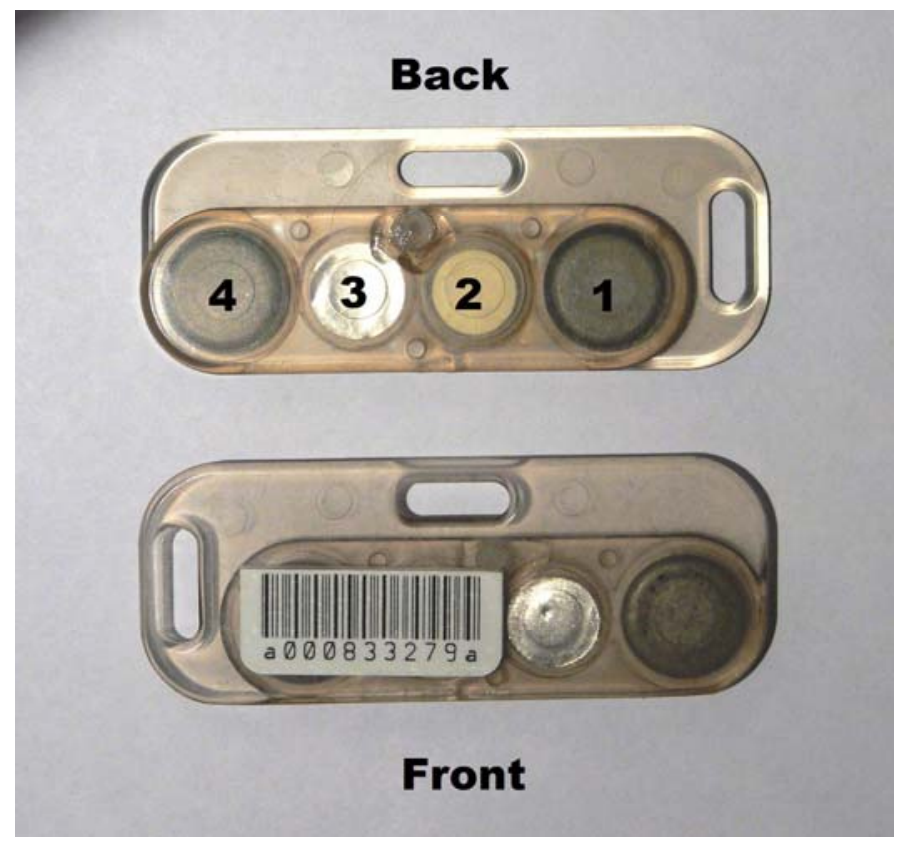

Figure 5.39 Hanford Personal Nuclear Accident Dosimeter

\subsubsection{Performance and Placement Criteria}

NAD performance criteria are provided in the DOE Radiological Control Standard (DOE 1999) as follows:

- Be capable of determining neutron dose in rad with an accuracy of $\pm 25 \%$ from 10 rads to approximately 10,000 rads.

- Be capable of providing the approximate neutron spectrum for conversion of rad to rem. 
- $\quad$ Be capable of measuring fission gamma radiation from 10 rads to approximately 10,000 rads in the presence of neutron radiation with an accuracy of approximately $\pm 25 \%$.

PNAD performance criteria are provided in the DOE Radiological Control Standard (DOE 1999), as follows:

- $\quad$ Be capable of measuring an absorbed dose in or on a phantom from 10 rads to approximately 1,000 rads with an accuracy of $\pm 25 \%$.

Criteria for FNAD placement have been adopted through the HPDAC. ${ }^{\text {(a) }}$ Guidance on the placement of FNADs is available in:

- ANSI/ANS 8.3-1986 (ANSI 1986) where the "minimum accident of concern" with nominal shielding is defined to result in a dose of approximately 20 rad in the first minute at a distance of 2 meters.

- $\quad$ ANSI N13.3-1981 (ANSI 1981) provides basic requirements for nuclear criticality dosimetry systems.

Because the potential dose to workers is highly dependent upon circumstances, only general FNAD placement criteria are provided, as follows:

- FNADs should be placed close to the actual work locations with minimal intervening shielding to allow for accurate measurement of dose consistent with DOE nuclear accident dosimetry performance criteria (DOE 1998c).

- Additional FNADs should be placed at greater distances from the radioactive source to allow extrapolation of dose to nearby workers or workers during egress.

- A system of worker-assigned personnel dosimeters and PNADs should be used to permit extrapolation of FNAD data to exposed workers.

- Provision should be available to determine the orientation of exposed workers based on dosimeter and/or biological measurement data.

- The background neutron dose rate at the FNAD location should generally not exceed $20 \mathrm{mrem} / \mathrm{h}$ or $175 \mathrm{rem} / \mathrm{y}$. To monitor locations where neutron dose rates exceed $20 \mathrm{mrem} / \mathrm{h}$, the FNADs should be positioned at a distance sufficient to reduce the dose rates to the prescribed dose rate levels or conduct a more frequent candlestick exchange.

- The background gamma radiation exposure rate at the FNAD location should not exceed $3 \mathrm{mR} / \mathrm{h}$ or $25 \mathrm{R} / \mathrm{y}$. For areas with an exposure rate exceeding $3 \mathrm{mR} / \mathrm{h}$, a more frequent candle exchange should be conducted.

(a) D. E. Bihl, "Minutes of the Hanford Personnel Dosimetry Advisory Committee Meeting Held December 3, 1997." Copies of HPDAC minutes are retained in Hanford Radiological Records historical file. 
- FNADs should be placed where they can be easily retrieved, and where the shielding and obstruction between the dosimeter and the potential radiation source are minimal. For example, FNADs may be placed in a hallway near the room to be monitored if the shielding provided between the hallway and the room is nominal. If this location is not practical, consider placing FNADs near a doorway to facilitate retrieval.

- For large sources (e.g., dimensions $>8 \mathrm{~m}$ ), FNADs should be positioned on approximate $15 \mathrm{~m}$ centers, with each FNAD within approximately $2 \mathrm{~m}$ of the source material.

- FNADs should be exchanged annually, the dose observed on the TLDs calculated, and the results analyzed to ensure compliance with the foregoing criteria.

\subsubsection{Quick-Sort Data Analysis}

Quick-sort is a field method for identifying individuals who have received significant neutron dose during a criticality accident. It is based on measurement of ${ }^{24} \mathrm{Na}$ activity in the human body. The activity is produced by activation of naturally occurring ${ }^{23} \mathrm{Na}$ in the body by thermal neutron capture. Although the incident neutrons may be primarily fast in a criticality incident, the moderating effect of the human body is sufficient to produce significant amounts of ${ }^{24} \mathrm{Na}$. Between $15 \%$ and $33 \%$ of the incident neutrons are thermalized and captured in the body (Cross and Ing 1985). ${ }^{24} \mathrm{Na}$ decays with a 15 hour half life and emits energetic gamma rays (1.37 MeV and $2.74 \mathrm{MeV}$ with $99 \%$ abundance) that are easily detected by most survey instruments.

Measurements can be made by placing a pancake GM probe against the abdomen of the subject who is bent over during the measurement. An alternative approach is to place the GM probe in the armpit and have the individual hold it tightly against his rib cage with his arm. Care should be taken to rule out possible instrument response to contamination by making a measurement with the probe window facing the body and another with the probe facing away from the body and comparing results, or by covering the window of the GM detector with suitable beta shield.

For a pancake GM probe, neutron dose in rad may be estimated as follows:

$$
\text { Dose }(\mathrm{rad})=2.2 \mathrm{C} / \mathrm{W}
$$

where $\mathrm{C}$ is the net count rate in counts per minute decay corrected to the time of the accident, and $\mathrm{W}$ is the subject's weight in pounds. Using this formula, a neutron dose of 1 rad would produce a net reading of $91 \mathrm{cpm}$ immediately after exposure on an individual weighing 200 pounds. Since "quick" sort methods are typically applied within a few hours of the accident, decay corrections can be ignored.

This formula is based on experimental measurements made on a saline filled phantom with a hard wall GM instrument after irradiation with $2.1 \mathrm{MeV}$ (avg) neutrons (Wilson, 1962). As shown here, the formula has been adjusted for 
differences in gamma sensitivity between the hard wall GM probe used in the experiment and the pancake GM probes used at Hanford today. A comparable formula for use with exposure rate instruments can be derived from the information in the Wilson study.

For an exposure rate instrument, absorbed neutron dose in rad may be estimated as follows:

$$
\text { Dose }(\mathrm{rad})=8000 \mathrm{R} / \mathrm{W}
$$

where $\mathrm{R}$ is the measured net exposure rate in $\mathrm{mR} / \mathrm{h}$ decay corrected to the time of the accident, and $\mathrm{W}$ is the subject's weight in pounds. Based on this formula, a neutron dose of 100 rads would produce a net reading of $2.5 \mathrm{mR} / \mathrm{h}$ immediately after exposure on an individual weighing 200 pounds. Since "quick" sort methods are typically applied within a few hours of the accident, decay corrections can be ignored.

Table 5.30 Survey Instrument Readings on the Body after an Accident

\begin{tabular}{||c|c|c|c|c|c|c||}
\hline \multirow{3}{*}{$\begin{array}{c}\text { Weight of } \\
\begin{array}{c}\text { Subject } \\
\text { (Pounds) }\end{array}\end{array}$} & \multicolumn{2}{|c|}{$\begin{array}{c}\text { Pancake GM Net Reading (cpm) } \\
\text { for a 10 rad Neutron Dose }\end{array}$} & \multicolumn{3}{c||}{$\begin{array}{c}\text { RO-3 Net Reading (mR/h) } \\
\text { for a 100 rad Neutron Dose }\end{array}$} \\
\cline { 2 - 7 } & $\begin{array}{c}\text { Immediately } \\
\text { After } \\
\text { Exposure }\end{array}$ & $\begin{array}{c}4 \text { Hours } \\
\text { After } \\
\text { Exposure }\end{array}$ & $\begin{array}{c}15 \text { Hours } \\
\text { After } \\
\text { Exposure }\end{array}$ & $\begin{array}{c}\text { Immediately } \\
\text { After } \\
\text { Exposure }\end{array}$ & $\begin{array}{c}\text { 4 Hours } \\
\text { After } \\
\text { Exposure }\end{array}$ & $\begin{array}{c}\text { 15 Hours } \\
\text { After } \\
\text { Exposure }\end{array}$ \\
\hline 125 & 568 & 472 & 284 & 1.6 & 1.3 & 0.8 \\
\hline 150 & 682 & 567 & 341 & 1.9 & 1.6 & 0.9 \\
\hline 175 & 795 & 661 & 398 & 2.2 & 1.8 & 1.1 \\
\hline 200 & 909 & 756 & 455 & 2.5 & 2.1 & 1.2 \\
\hline 225 & 1023 & 850 & 511 & 2.8 & 2.3 & 1.4 \\
\hline 250 & 1136 & 945 & 568 & 3.1 & 2.6 & 1.6 \\
\hline
\end{tabular}

The data in Table 5.30 are based on Equations 5.16 and 5.17. It should be noted that absorbed dose values determined from these formulae represent only the neutron first collision dose. The dose from gamma rays produced by capture reactions within the body is not included. Depending on the incident neutron energy spectrum, capture gammas may contribute as much as $50 \%$ of the total absorbed dose attributable to the incident neutrons (Takahashi, Endo and Yamaguchi, 2003). It should also be noted that the above relationships and the data shown in Table 5.30 are based only on ${ }^{24} \mathrm{Na}$ activity in the body and do not account for activated ${ }^{38} \mathrm{Cl}$ present during the first four hours after exposure. Capture of thermal neutrons by ${ }^{37} \mathrm{Cl}$ produces ${ }^{38} \mathrm{Cl}$ which decays with a 37 minute half life, and emits $2.17 \mathrm{MeV}$ gammas (47\% abundance) and $1.64 \mathrm{MeV}$ gammas (38\% abundance). Immediately following exposure, approximately $50 \%$ of the blood activity will be from ${ }^{38} \mathrm{Cl}$ (Hankins 1980). Because ${ }^{38} \mathrm{Cl}$ activity is not accounted for in the above formulae, the error in dose estimates based on these formulae can be as large as $50 \%$ for measurements made immediately after an accident (Mettler and Voelz, 2001) but becomes insignificant for measurements made two or more hours after exposure. 
Large uncertainties are inherent in dose estimates obtained with the formulae above. In addition to the unaccounted for capture gamma dose and interference from ${ }^{38} \mathrm{Cl}$, uncertainties in the incident neutron energy spectrum can cause errors as large as a factor of 3 (Takahashi, Endo and Yamaguchi, 2003), and uncertainties in orientation of the body within the field can cause errors as large as a factor of 1.8 (Cross, 1981). The formulae above are based on unmoderated neutrons and AP exposure geometry. To the extent that actual exposure conditions involve scattered neutrons (particularly from the floor), the above formulae will tend to overestimate absorbed dose. To the extent that exposure is from the side rather than front of the body, the above formulae will tend to underestimate the absorbed dose. Therefore, these two errors tend to counteract each other. Nevertheless, given all the uncertainties involved at the time of a quick sort, any dose estimate obtained with the above methods should be considered a rough approximation, with potential error as large as a factor of 5 .

\subsubsection{Analysis of Physical and Biological Samples}

Analysis of Metallic

Samples

Analysis of Biological Samples

Blood Sodium Dose Conversion Factors
Neutron radiation present in any criticality event will activate nearby physical and biological materials, depending on the composition (i.e., atomic elements) of the material. Once activated, these materials are radioactive. Analysis of these radioactive materials provides evidence of the fluence and energy of neutron radiation resulting from the criticality. Knowledge of the neutron fluence and energy spectrum enables the dose to personnel near the criticality event to be estimated.

Metal objects carried by employees can be good indicators of exposure to neutron radiation due to activation of the metal. If samples of metallic objects carried by the person (coins, buckles, eyeglass frames, etc.) are submitted to HEDP staff, the samples can be counted using gamma spectroscopic capabilities, and an assessment of neutron exposure can be made.

Standard man (70 kg) contains about $100 \mathrm{~g}$ of ${ }^{23} \mathrm{Na}$ (ICRP 1974). By neutron activation, the sodium is transformed into radioactive ${ }^{24} \mathrm{Na}$, which emits an energetic gamma ray that can be easily detected. Depending on the type of technique employed, concentrations as low as $3.9 * 10^{-5} \mu \mathrm{Ci} / \mathrm{ml}$ with a 30 -minute counting time or $9.4 * 10^{-5} \mu \mathrm{Ci} / \mathrm{ml}$ with a 10 -minute counting time can be measured. Similarly, hair samples can be analyzed for ${ }^{32} \mathrm{P}$, produced by activation of ${ }^{32} \mathrm{~S}$, to determine an employee's fast neutron exposure (i.e., energy $>2.9 \mathrm{MeV}$ ). Hair samples are particularly good to determine orientation of the body during exposure if hair samples can be obtained from different locations. Analysis of blood for chromosome aberration may be a useful technique to assist in the estimation of total dose.

The following neutron dose conversion factors $(\mathrm{K})$ for blood sodium activity were empirically determined during simulated blood sodium experiments at the HPRR at ORNL (doses are given in tissue kerma):

Bare spectrum: $\quad \mathrm{K}=0.168 \pm 0.004 \mathrm{rad} / \mathrm{dpm}-\mathrm{mg}$

Steel shield: $\quad K=0.145 \pm 0.006 \mathrm{rad} / \mathrm{dpm}-\mathrm{mg}$ 


$$
\begin{array}{ll}
\text { Concrete shield: } & \mathrm{K}=0.116 \pm 0.116 \mathrm{rad} / \mathrm{dpm}-\mathrm{mg} \\
\text { Lucite }{ }^{\mathrm{TM}} \text { shield: } & \mathrm{K}=0.088 \pm 0.007 \mathrm{rad} / \mathrm{dpm}-\mathrm{mg}
\end{array}
$$

\subsubsection{Interpretation of Personnel Dosimeter Results After a Criticality Event}

Analysis of personnel dosimeters for all employees involved in a criticality will be conducted quickly following any criticality. Typically, normal procedures are used to initially estimate the radiation dose measured by each dosimeter.

Additional interpretation is made when dose and spectrum measurements become available from the FNAD nearest to the event location and the PNADs worn by affected staff. When evaluating the HSD or HCND results after a criticality, the dose and spectrum information obtained from the PNADs and FNADs allows corrections to be made to the reported neutron dose and dose equivalent. It is expected that each PNAD and FNAD will provide different information because of the location of the respective FNADs and affected workers' positions and movements during the criticality event. Interpretation of dose for each affected person will be necessary on a case-by-case basis.

Laboratory measurements using progressive levels of moderation in ${ }^{252} \mathrm{Cf}$ and $\mathrm{PuF}_{4}$ spectra, have shown that the TLD albedo capability in the HCND can estimate dose with reasonable accuracy over a wide range of neutron spectra.

\subsubsection{Assessment of Dose After a Criticality Event}

Early estimates of the severity of an exposure to prompt radiation emitted by a criticality event are estimated based on results of portable survey measurements, personnel dosimeters, and in vivo bioassay measurements. Parameters and dose conversion factors used to determine the dose from PNADs and FNADs are generally based on prior calibration and/or intercomparison testing of Hanford PNADs, FNADs, or data from NCRP Report No. 57 (1978) and the International Atomic Energy Agency Technical Report No. 211 (IAEA 1982).

Later estimates of dose will be based on many additional measurements to confirm and further quantify the neutron, photon, and total doses received by exposed individuals. Measurements likely to be available include the following:

- $\quad$ additional analysis of personnel dosimeter response characteristics

- $\quad$ analysis of PNADs and FNADs

- $\quad$ additional analysis whole body counts for ${ }^{24} \mathrm{Na}$ activation

- $\quad$ blood sample analysis for ${ }^{24} \mathrm{Na}$ activation

- $\quad$ hair sample analysis for ${ }^{32} \mathrm{P}$ activation

- $\quad$ chromosome aberration analysis.

Analysis of dose based on results from HSDs, HCNDs, and PNADs is the preferred method of determining dose because the dosimeters are worn by the person and dosimeter response data for specific neutron spectra are available. Results from FNADs located nearby may be used to provide estimates of dose in the cases where results of the personnel dosimeters and PNADs are compromised because of shielding, etc., and cannot be directly used. In vivo and in vitro (blood) measurements of ${ }^{24} \mathrm{Na}$ activation should be performed within 2- to 24 hours of the exposure, whereas the ${ }^{32} \mathrm{P}$ can be counted and analyzed several days 
Gamma Ray Dose

Neutron Dose

Chromosome Aberration after the exposure without compromising detection levels and accuracy. In general, it is important to recognize that there is a trade-off between the promptness by which the laboratory analyses of neutron activation samples can be made and the accuracy of the results. Chromosome aberration analysis should be considered when preliminary dose estimates exceed $10 \mathrm{rad}$.

Response protocols are expected to vary according to the type of measurement and analysis required and the likely severity of the exposure, as indicated by results from quick-sort surveys, personnel dosimeters, and in vivo counts.

The gamma ray dose is determined from the personnel TLD. The TLDs are processed and analyzed in accordance with standard TLD procedures. The gamma ray dose estimated from the personnel dosimeters may need to be corrected for attenuation through the body if the individual was facing away from the source of the exposure. This determination is based upon hair sulfur activation results and interviews with the victims.

In the case of nuclear events, acute biological effects are predominant and quality factors are not relevant. Neutron dose should be assessed in rad and should refer to the maximum absorbed dose due to incident neutrons. The quick-sort procedure and the whole body count provide estimates of neutron dose only. Early estimates of the neutron dose may also be obtained by other means, such as results from Hanford standard and/or combination neutron personnel dosimeters, and the PNAD.

Neutron dose assessment procedures for HSDs, HCNDs, PNADs, and FNADs are maintained in PNL-MA-841, Hanford External Dosimetry Procedures Manual.

Chromosome aberration analysis may be a useful technique to assist in the estimation of total dose after a nuclear event. However, the amount of chromosome damage produced in human blood lymphocytes depends on the gamma to neutron dose ratio and the gamma dose rate. Chromosome aberration analysis is recommended if an exposure of $>5$ rad is indicated by in-vivo analysis, blood, or hair radiochemical analyses. Chromosome aberration analysis should be considered when preliminary dose estimates exceed $10 \mathrm{rad}$.

\subsection{Useful Dose Range for Hanford Dosimeters}

The useful dose range is defined as the range of delivered dose for which meaningful dose measurement can be made with a dosimeter that is routinely processed without special procedures. For any given type and energy of radiation, this range may be considered to extend from the lower limit of detection to the dose that produces the maximum possible PMT current without substantial nonlinearity (e.g. loss of signal from saturation). [With advanced notice that a large dose has been delivered to the dosimeter, special measures such as reduced PMT gain, slower heating, or use of neutral density filters can be implemented to extend the range of dose interpretation by an order of magnitude or more up to a maximum of about 50,000 rads, beyond which, the supralinearity correction function becomes double valued.] The point at which the PMT is delivering maximum possible current and beyond which loss of signal begins, is evidenced by a clipping or flattening of the top of the glow curve. For the standard $1 / 2$ inch diameter PMT used in Harshaw 6600 and 8800 TLD readers, with high voltage 
set to achieve a nominal sensitivity of approximately $0.2 \mathrm{nC} / \mathrm{mR}$, the maximum integrated current from a normal shaped glow curve was determined to be approximately $442 \mu \mathrm{C}$. The nominal sensitivity is based on readout of oven annealed $100 \mathrm{mg} / \mathrm{cm}^{2}$ TLD 700 chips using standard TTP, per standard HEDP reader calibration practice. Using the upper limit of useful PMT response, and the fundamental response characteristics of HEDP dosimeter elements to various types and energies of radiation at normal occupational dose levels, the delivered doses for each type and energy of radiation corresponding to PMT saturation were determined for each dosimeter type. Based on this analysis, a summary of the maximum measurable personal dose equivalent (rounded to the nearest $100 \mathrm{rem}$ ) for selected HEDP dosimeters and radiation types is given in Table 5.31. The accuracy that is theoretically obtainable at these dose levels is $\pm 30 \%$ at a onesigma confidence level. A more complete set of results with details of the measurements and calculations is documented in HEDP files. ${ }^{\text {(a) }}$

Table 5.31 Maximum Measurable Dose for Hanford Dosimeters

\begin{tabular}{|c|c|c|c|c|c|}
\hline \multicolumn{2}{|c|}{ Radiation } & \multicolumn{3}{c|}{ Maximum Dose } \\
\hline $\begin{array}{c}\text { Radiation } \\
\text { Type }\end{array}$ & Source & $\begin{array}{c}\text { Average } \\
\text { Energy } \\
(\mathrm{keV})\end{array}$ & $\begin{array}{c}\text { HSD } \\
\text { Deep } \\
\text { rem }\end{array}$ & $\begin{array}{c}\text { HCND } \\
\text { Deep } \\
\text { rem }\end{array}$ & $\begin{array}{c}\text { Ring } \\
\text { Shallow } \\
\text { rem }\end{array}$ \\
\hline x-ray & NIST M30 Technique & 20 & 500 & 500 & 1400 \\
\hline x-ray & NIST M150 Technique & 73 & 1000 & 1000 & 1400 \\
\hline x-ray & NIST H150 Technique & 118 & 1100 & 1100 & 1600 \\
\hline gamma & ${ }^{137} \mathrm{Cs}$ & 662 & 1200 & 1200 & 1900 \\
\hline beta & ${ }^{90} \mathrm{Y}$ & 931 & - & - & 1800 \\
\hline beta & ${ }^{204} \mathrm{TI}$ & 267 & - & - & 8300 \\
\hline neutrons & Unmoderated ${ }^{252} \mathrm{Cf}$ & 2100 & 2100 & 2100 & - \\
\hline neutrons & $\mathrm{D}_{2} \mathrm{O}$ Moderated ${ }^{252} \mathrm{Cf}$ & 550 & 300 & 300 & - \\
\hline
\end{tabular}

For the HSD, the maximum measurable personal dose equivalent at all depths (shallow, eye and deep) are provided in Table 5.32. To determine the maximum measurable shallow, eye or deep personal dose equivalent for a mixture of radiation types where the relative contribution of each type to the total dose is known, Equation 5.18 may be used in conjunction with the data in Table 5.32.

$$
H_{\max }=\frac{1}{\sum_{i=1}^{n} \frac{w_{i}}{L_{i}}}
$$

(a) B. A. Rathbone, “Useful Range of Hanford Dosimeters,” September 25, 2002, letter to HEDP file. 
Where:

$$
\begin{array}{ll}
H_{\max } & =\quad \text { maximum measurable personal dose equivalent for the mixture } \\
w_{i} & =\quad \text { fraction of total dose contributed by pure radiation type } i \\
L_{i} & =\quad \text { maximum measurable dose (limit) for pure radiation type } i \\
n & =\quad \text { number of radiation types in the mixture }
\end{array}
$$

\begin{tabular}{|c|c|c|c|c|c|}
\hline \multicolumn{3}{|c|}{ Radiation } & \multicolumn{3}{|c|}{ Maximum Measurable Dose (rem) } \\
\hline Radiation Type & Source & $\begin{array}{c}\text { Average } \\
\text { Energy } \\
\text { (keV) }\end{array}$ & Shallow & Eye & Deep \\
\hline photon & M30 & 20 & 1151 & 830 & 478 \\
\hline photon & M60 & 34 & 1008 & 960 & 837 \\
\hline photon & S60 & 38 & 914 & 914 & 866 \\
\hline photon & M100 & 51 & 872 & 885 & 886 \\
\hline photon & Am-241 & 59 & 849 & 910 & 915 \\
\hline photon & M150 & 73 & 904 & 939 & 980 \\
\hline photon & $\mathrm{H} 150$ & 118 & 1034 & 1061 & 1108 \\
\hline photon & Cs-137 & 662 & 1178 & 1179 & 1178 \\
\hline beta & Y-90 & 931 & 1668 & 741 & \\
\hline beta & TI-204 & 267 & 3036 & & \\
\hline neutrons only & Cf-252 U & 2100 & & & 2117 \\
\hline neutrons only & Cf-252 M & 550 & & & 285 \\
\hline neutron + photon & Cf-252 U & 2100 & 110 & 110 & 2131 \\
\hline neutron + photon & Cf-252 M & 550 & 34 & 33 & 313 \\
\hline
\end{tabular}

NOTE: For any given mixture, $H_{\max }$ must be calculated independently for shallow dose, eye dose and deep dose.

Table 5.32 Maximum Measurable Dose for HSD at Various Depths.

The shaded values for neutron deep personal dose equivalent in Table 5.31 and Table 5.32 have been updated to reflect changes in neutron fluence to dose equivalent conversion factors for $\mathrm{H}_{\mathrm{p}}(10)_{\eta}$ that follow from the revised Q-LET relationship given in ICRP 60 (a) $^{\text {(a) }}$ The new maximum neutron dose values are based on the neutron dose rates for the bare and moderated ${ }^{252} \mathrm{Cf}$ calibration sources at PNNL that have been determined using the ICRP 60 based fluence to dose equivalent conversion factors that have been published in ICRP 74.

(a) B. A. Rathbone, "Re-evaluation of Useful Dose Range for HSD and HCND,” August, 11, 2009, letter to HEDP file. 


\subsection{Operational Considerations}

The operational practices involved in the administration of an external dosimetry program that are described in this chapter have been developed for use at Hanford. As such, they have been reviewed and concurred in by Hanford radiological control organizations through the HPDAC (described in Chapter 2). Non-Hanford users can subscribe to the Hanford practices as documented here or document their own practices in a site-specific document. To ensure accurate measurement of dose, the practices described here regarding how the dosimeters should be used, are strongly recommended for non-Hanford users as well. Other practices such as selection of persons to be monitored are documented here to support the RPPs of Hanford contractors. Such practices should be documented by the non-Hanford user to comply with their own regulatory and other requirements.

Hanford contractor radiation protection organizations are responsible for field dosimetry practices, including monitoring of exposure conditions in the work environment, controlling worker dose, and properly using HEDP dosimetry and technical support. Radiation protection organizations select the personnel to be monitored, the type of dosimeter to be used, the exchange frequency, and the facility calibration code to use for processing. They maintain portable instrument survey data of the work environments, and conduct dose evaluations for any lost or missing dosimeter for their personnel. They are also responsible for the contractor-specific ALARA programs and Area Monitoring programs. HEDP Dosimetrists work closely with contractor radiation protection organizations regarding several operational aspects of the dosimetry program including technical support for special dosimetry applications, dose investigations and identifying the need for field specific correction factors and/or dose algorithms where necessary. HEDP is responsible for maintaining detailed records of dosimeter processing activities, including dosimeter raw data and results, dose assessments, QA, QC, training, staff qualifications, equipment maintenance and calibration.

\subsection{Occupational Dose}

DOE requires the dosimetry program to assess personnel dose resulting from occupational exposure. Dose from medical procedures or from natural background radiation is not to be included in the recorded dose. To help achieve this objective, Hanford worker training includes the statement that personnel are to contact their respective radiation protection organization representative whenever there is a possibility of dose from non-occupational circumstances, such as medical procedures. In these cases, the radiation protection representative together with the person's supervisor will develop an approach to ensure that non-occupational dose is not recorded.

Similarly, Hanford worker training includes instruction to not wear dosimeters while having medical procedures performed (e.g. x-rays, nuclear medicine) or to take dosimeters with them while on travel (e.g. in checked baggage or carry-on baggage submitted to x-ray security screening devices). As part of the external 
dose calculation methodology, Hanford background functions are used to compensate for the dosimeter response from naturally occurring environmental radiation (see Chapter 5). For offsite customers, this includes the use of site specific background functions and the use of transit control dosimeters in dosimeter shipments to measure abnormal transit dose.

\subsection{Selection of Individuals to be Monitored}

In accordance with 10CFR835.402(a) (DOE 2007a),

For the purpose of monitoring individual exposures to external radiation, personnel dosimeters shall be provided to and used by:

(1) Radiological workers who, under typical conditions, are likely to receive a dose greater than or equal to one or more of the following ${ }^{(a)}$ in a year:

- effective dose of $0.1 \mathrm{rem}(0.001 \mathrm{~Sv})$

- equivalent dose to the lens of the eye of $1.5 \mathrm{rems}(0.015 \mathrm{~Sv})$

- equivalent dose to the skin of 5 rems $(0.05 \mathrm{~Sv})$

- equivalent dose to any extremity of 5 rems $(0.05 \mathrm{~Sv})$

(2) Declared pregnant workers who are likely to receive from external sources an equivalent dose to the embryo/fetus in excess of $50 \mathrm{mrem}(0.5 \mathrm{mSv})$ from the period of conception to birth;

(3) Occupationally exposed minors who are likely to receive from external sources a dose in excess of one or more of the following in a year:

- effective dose of $50 \mathrm{mrem}(0.5 \mathrm{mSv})$

- equivalent dose to the lens of the eye of $750 \mathrm{mrem}(7.5 \mathrm{mSv})$

- equivalent dose to the skin of $2.5 \mathrm{rem}(25 \mathrm{mSv})$

- equivalent dose to any extremity of $2.5 \mathrm{rem}(25 \mathrm{mSv})$

(4) Members of the public entering a controlled area likely to receive from external sources an effective dose in excess of $50 \mathrm{mrem}(0.5 \mathrm{mSv})$ in a year:

(5) Individuals entering a high or very high radiation area.

In accordance with the Hanford Radiological Health and Safety Document (DOE 2001), personnel dosimeters shall be provided to and used by:

(1) Non-occupationally exposed minors entering RBAs or RMAs

Additional dosimeters may be issued as contractor radiation protection organizations deem appropriate. Contractor radiation protection organizations are responsible for assigning and exchanging dosimeters and determining the type of dosimeter to be assigned in accordance with the guidance in this manual.

(a) The wording 10CFR835.402(a)(1) does not explicitly state that the doses to be considered are from external sources only. However DOE-STD-1098-2008 Part 511.1.adds the words "from external sources" and DOE G 441.1-1C implies that these doses are from external sources only. 


\subsection{Selection of Dosimeter Types to Use}

Two types of whole body dosimeter (HSD and HCND) are available for issue to individuals at Hanford. The HSD is designed to measure shallow, eye, and deep personal dose equivalent from beta and photon radiation fields $\left[\mathrm{H}_{\mathrm{p}}(0.07), \mathrm{H}_{\mathrm{p}}(3)\right.$, $\left.\mathrm{H}_{\mathrm{p}}(10)_{\gamma}\right]$. In addition, the dosimeter has a neutron-sensitive TLD-600 phosphor for neutron detection. Although not intended as the primary dosimeter for measuring neutron dose, the HSD has been DOELAP accredited in neutron exposure categories and may be used for limited monitoring of individuals who are not likely to receive a deep personal dose equivalent from neutrons, $H_{p}(10)_{\eta}$ greater than 100 mrem per year. Individuals who are likely to receive $H_{p}(10)_{\eta}$ greater than 100 mrem per year should be issued a HCND, which provides a more accurate measurement of neutron dose. In addition, individuals who routinely have $\mathrm{H}_{\mathrm{p}}(10)_{\eta}$ greater than 100 mrem per year reported on an HSD should be issued a HCND. The HSD generally provides a conservative measure of neutron dose. For most applications, which typically involve a large component of scattered neutrons, the HSD results tend to be excessively conservative (factor of 4 or more), increasing the potential for exceeding administrative control levels based on reported dose results when significant neutron exposure is involved. The HCND offers improved accuracy for betagamma dosimetry in the presence of neutrons as well as improved neutron dosimetry. The features and capabilities of the HSD and HCND are discussed in Section 5.4 and Section 5.5 respectively.

Two types of extremity dosimeter are DOELAP accredited and available for routine use at Hanford (EXT-RAD ring dosimeter, and HSD Wrist/Ankle dosimeter). The design features and response characteristics of these dosimeters are discussed in detail in Chapter 5.

The EXT-RAD is suitable for use in PFP photon-neutron fields when processed with the default calibration code 00 (applies calibration factor $=2.0$ ). For use in other photon-neutron fields, a field specific correction factor will need to be determined to compensate for the undetected neutron dose. The HSD Wrist/Ankle dosimeter is useful for monitoring forearms and ankles, and for general extremity monitoring when beta and photon energies are largely unknown or rudimentary detection and measurement of neutrons is desired.

Dosimetry for specialized applications is also available upon request. An example is the chipstrate used as a band-aid type dosimeter (i.e. without EXTRAD strap) for monitoring body locations that are difficult to attach routine dosimetry to (e.g. soles of feet, tips of fingers, eyes). Another example is an EXT-RAD loaded with a neutron sensitive element.

\subsection{Dosimetry Limitations}

HEDP dosimetry processing methods and dose calculation algorithms are based primarily on laboratory measurements with traceable calibration sources. However, because of limitations in dosimetry technology, laboratory based algorithms are not capable of providing accurate results for all possible field conditions. Because actual field exposure geometries and energy spectra are 
sometimes difficult to simulate in the laboratory, workplace measurements are sometimes necessary to establish field specific correction factors and/or dose algorithms. Such correction factors and algorithms have already been established for some Hanford applications. However, all possible dosimetry applications at Hanford that may warrant use of special dose algorithms and/or correction factors have not been identified or addressed in this manual. In addition, because of changes in field practices or workplace conditions, existing field specific correction factors and algorithms need to be re-evaluated from time to time. Therefore, Hanford contractor radiation protection organizations should have a mechanism for identifying workplace conditions and dosimetry applications within their facilities that fall outside the established capability of the Hanford dosimetry system and for identifying changes in practices or workplace conditions that may invalidate field specific correction factors and algorithms currently in use. Information in this manual regarding the response characteristics, capabilities and limitations of the various dosimeter designs has been provided for this purpose. Hanford contractor radiation protection organizations should request technical support from HEDP as necessary to meet this objective. Table 6.1 summarizes the intended applications and limitations for Hanford dosimeters and identifies general types of applications requiring special calibration factors.

\subsection{Dose Reporting Threshold}

Established dose reporting thresholds are applied by dose reporting software to the calculated dose results that reside in the ED database. (Calculated dose results in the ED database may be any real number greater than or equal to zero.) The dosimeter dose reporting thresholds are shown in Table 6.2 below. These levels are less than $1 \%$ of the respective DOE equivalent dose limits. Doses are reported to the nearest mrem (i.e., 11, 12, 111, 1112, etc.). In the case of the $\mathrm{HCND}, \mathrm{H}_{\mathrm{p}}(0.07), \mathrm{H}_{\mathrm{p}}(3)$, and $\mathrm{H}_{\mathrm{p}}(10)_{\gamma}$ are reported by the 8825 component and $\mathrm{H}_{\mathrm{p}}(10)_{\eta}$ is reported by the 8816 component independently (i.e., a separate record).

It should be noted that the calculated LLDs for a given dosimeter type vary with radiation type, depth of interest, and wear period (see Chapter 5). Typically, radiation types for which the dosimeter is less sensitive, produce larger LLDs. Longer wear periods generally correspond to larger LLDs.

The Hanford practice for use of reporting thresholds was discussed extensively by the HPDAC. ${ }^{\text {(a) }}$ The basic issue centered around whether to use multiple reporting thresholds corresponding to detection thresholds, or use a simple threshold corresponding to practice at some other DOE sites and past practice at Hanford, or use no thresholds at all. The question of which statistical concept

(a) D. E. Bihl, "Minutes of the Hanford Personnel Dosimetry Advisory Committee Meeting Held on February 23, 1999.

D. E. Bihl, "Minutes of the Hanford Personnel Dosimetry Advisory Committee Meeting Held on March 23, 1999.

D. E. Bihl, "Minutes of the Hanford Personnel Dosimetry Advisory Committee Meeting Held on July 13, 1999.

D. E. Bihl, "Minutes of the Hanford Personnel Dosimetry Advisory Committee Meeting Held on August 17, 1999.

D. E. Bihl, "Minutes of the Hanford Personnel Dosimetry Advisory Committee Meeting Held on September 21, 1999.

D. E. Bihl, "Minutes of the Hanford Personnel Dosimetry Advisory Committee Meeting Held on October 12, 1999. 
$\left(\mathrm{L}_{\mathrm{C}}, \mathrm{L}_{\mathrm{D}}, \mathrm{L}_{\mathrm{Q}}\right.$, or other) would be appropriate to use for a reporting threshold was considered. This nomenclature was first proposed by Lloyd Currie (Currie 1968) and is still widely used today. Questions of what probability for type I and type II errors would be acceptable were discussed. Questions of "unreported dose" were considered. Potential impacts of changes in threshold on collective dose reported for Hanford contractors were evaluated.

Table 6.1 Summary of Dosimeter Applications and Limitations ${ }^{(a)}$

\begin{tabular}{|c|c|c|c|c|}
\hline Dosimeter Type & Radiations Measured & Intended Application & Precautions & Limitations \\
\hline HSD & $\begin{array}{l}\text { photons } 16 \mathrm{keV}-5 \mathrm{MeV} \text {, } \\
\text { beta } 250 \mathrm{keV}-2.2 \mathrm{MeV} \\
\text { neutrons } 0.025 \mathrm{eV}-5 \mathrm{MeV}\end{array}$ & $\begin{array}{l}\text { Routine beta-gamma } \\
\text { dosimetry, limited low dose } \\
\text { neutron dosimetry. }\end{array}$ & $\begin{array}{l}\text { Dosimeter under-responds to } \\
\text { betas with energies }<250 \mathrm{keV} \text {. } \\
\text { Dosimeter significantly over- } \\
\text { responds to moderated neutrons. }\end{array}$ & $\begin{array}{l}\text { Should not be used for neutron } \\
\text { monitoring if neutron dose is expected to } \\
\text { be greater than } 100 \mathrm{mrem} / \mathrm{y} \text {. Special } \\
\text { facility cal code required if beta energy } \\
<250 \mathrm{keV} \text { average. }\end{array}$ \\
\hline HCND & $\begin{array}{l}\text { photons } 16 \mathrm{keV}-5 \mathrm{MeV} \text {, } \\
\text { beta } 250 \mathrm{keV}-2200 \mathrm{keV} \\
\text { neutrons } 0.025 \mathrm{eV}-5 \mathrm{MeV}\end{array}$ & $\begin{array}{l}\text { Routine beta-gamma } \\
\text { dosimetry, routine neutron } \\
\text { dosimetry at all Hanford } \\
\text { facilities }\end{array}$ & $\begin{array}{l}\text { Dosimeter under-responds to } \\
\text { betas with energies }<250 \mathrm{keV}\end{array}$ & $\begin{array}{l}\text { Special facility calibration code is } \\
\text { required if beta energy }<250 \mathrm{keV} \\
\text { average. }\end{array}$ \\
\hline $\begin{array}{l}\text { EXT-RAD } \\
\text { Ring }\end{array}$ & $\begin{array}{l}\text { photons } 16 \mathrm{keV}-5 \mathrm{MeV} \\
\text { beta }>200 \mathrm{keV}\end{array}$ & $\begin{array}{l}\text { Extremity monitoring for work } \\
\text { with mixed fission product } \\
\text { (MFP) waste or purified beta- } \\
\text { gamma isotopes. } \\
\text { Extremity monitoring for work } \\
\text { with Pu and Pu compounds at } \\
\text { PFP in applications where lead } \\
\text { content of gloves is less than } \\
\text { one half value layer for } 60 \mathrm{keV} \\
\text { photons. } \\
\text { Extremity monitoring in } \\
\text { neutron-gamma fields other } \\
\text { than at PFP when } \eta / \gamma \text { ratio } \leq \\
1.0\end{array}$ & $\begin{array}{l}\text { Dosimeter under-responds to } \\
\text { betas with energies }<200 \mathrm{keV} \text {. } \\
\text { Dosimeter does not respond to } \\
\text { neutrons. Default calibration } \\
\text { factor }=2.0 \text { adequately accounts } \\
\text { for neutron dose in } \eta-\gamma \text { fields } \\
\text { where } \eta / \gamma \text { ratio } \leq 1.0 \text { or } \eta-\gamma \\
\text { fields where } \eta / \gamma \text { ratio } \leq 2.0 \text { and } \\
\text { photon energies }<100 \mathrm{keV} \text {. } \\
\text { When used with leaded gloves } \\
\text { in neutron fields, the } \eta / \gamma \text { ratio } \\
\text { inside the glove must be } \leq 1 . \\
\text { Otherwise special field } \\
\text { calibrations may be necessary }\end{array}$ & $\begin{array}{l}\text { Special facility calibration code is } \\
\text { required if average beta energy is }<200 \\
\mathrm{keV} \text { and more than half of the extremity } \\
\text { dose is from beta radiation. } \\
\text { Special facility calibration code and/or a } \\
\text { neutron sensitive dosimeter (e.g. } \\
\mathrm{HSD} / \text { wrist) is required if } \eta / \gamma>1 \\
\text { Special facility calibration code is } \\
\text { required for work with Pu and Pu } \\
\text { compounds at PFP if lead content of } \\
\text { gloves is greater than one half value } \\
\text { layer for } 60 \mathrm{keV} \text { photons. }\end{array}$ \\
\hline $\begin{array}{c}\text { HSD } \\
\text { Wrist/Ankle }\end{array}$ & $\begin{array}{l}\text { photons } 16 \mathrm{keV}-5 \mathrm{MeV} \text {, } \\
\text { beta } 250 \mathrm{keV}-2200 \mathrm{keV} \\
\text { neutrons } 0.025 \mathrm{eV}-5 \mathrm{MeV}\end{array}$ & $\begin{array}{l}\text { Monitoring of wrists/ankles in } \\
\text { beta-gamma fields. Monitoring } \\
\text { in neutron fields with special } \\
\text { facility calibration code. }\end{array}$ & $\begin{array}{l}\text { Dosimeter under-responds to } \\
\text { betas with energies }<250 \mathrm{keV} \text {. } \\
\text { Dosimeter has energy } \\
\text { dependent response to neutrons. }\end{array}$ & $\begin{array}{l}\text { Special facility cal code required if beta } \\
\text { energy }<250 \mathrm{keV} \text { average. Special } \\
\text { facility cal code may be required for } \\
\text { neutron monitoring. }\end{array}$ \\
\hline
\end{tabular}

(a) The data in Table 6.1 apply to the given Dosimeter Type when the dosimeter is used in conjunction with the default facility calibration code. The facility calibration code is a two digit code entered into REX when the dosimeter is returned for processing. This code tells the external dosimetry dose calculation software which algorithm and/or which correction factor to apply when calculating dose. The default code applied by REX is 00 . The default facility calibration code has been established to support most routine applications at Hanford. Additional calibration codes can be set up as needed to support special applications involving low energy beta emitters and/or unique neutron monitoring requirements. 
A policy decision was made by DOE-RL (Radiological Control Steering Committee) to continue using the simplified scheme of 10 mrem thresholds already in place ${ }^{(a)}$ with the exception of neutron dose on 8816 TLDs (discussed below). Given the fact that the calculated LLDs are for the most part within 10 mrem of the reporting thresholds, regardless of exchange frequency or radiation type, the added complexity of applying multiple thresholds was not considered necessary. Therefore, in the interest of simplicity and consistency with past Hanford practice, a single reporting threshold has been adopted for each dose quantity and dosimeter type regardless of exchange period.

Because the LLD for neutron TLDs depends greatly upon the neutron energy spectra involved, an a priori reporting threshold is not applied to the calculated dose result. However, the 8816 algorithm does apply a threshold of sorts for calculation of dose. A minimum level of net neutron signal ( $\mathrm{mR}$ equivalent) is necessary on each of the three TLD 600 chips before meaningful element ratio analysis and dose calculation can be performed. If sufficient TL signal is not present, then the algorithm sets the "calculated" dose to zero mrem. Based on neutron energy spectra encountered in Hanford facilities, this calculation threshold equates to reported doses between approximately one and ten mrem. DOE-RL (Radiological Control Steering Committee) reviewed and approved this new approach to reporting thresholds for neutron dose on the HCND in September 1999. ${ }^{\text {(b) }}$ Removal of the a priori reporting threshold that had been applied to calculated neutron dose on HCNDs was implemented October 1, 1999.

The 2007 amendment to 10 CFR 835 (DOE 2007a) mandated the use of operational quantities consistent with the Q-LET given in ICRP 60. Specifically, the neutron quality factors used in the calculation of personal dose equivalent from neutrons have increased substantially. The impact on calculated LLDs for neutron dose was evaluated. The revised LLDs for neutrons increased $29 \%$ for unmoderated neutrons and $49 \%$ for moderated neutrons (based on assessed neutron dose rates for PNNL sources), but are still less than the reporting thresholds for $\mathrm{H}_{\mathrm{p}}(10)_{\eta}$ in Table 6.2. An evaluation was performed to determine if an increase in reporting thresholds for neutron dose was warranted in conjunction with implementation of the 2007 amendment to 10 CFR 835 . $^{(\mathrm{c})}$ The analysis showed that the previously established thresholds were still adequate and that a change was not warranted.

(a) D. E. Bihl, "Minutes of the Hanford Personnel Dosimetry Advisory Committee Meeting Held on March 23, 1999.

(b) D. E. Bihl, "Minutes of the Hanford Personnel Dosimetry Advisory Committee Meeting Held on September 21, 1999.

(c) B. A. Rathbone "Reporting Thresholds for HSD and HCND" August 11, 2009, Letter to HEDP file 
Table 6.2. Dose Reporting Thresholds (mrem)

\begin{tabular}{|l|c|c|c|c|c||}
\hline & HSD & $\begin{array}{c}\mathbf{8 8 2 5} \\
\text { HCND }\end{array}$ & $\begin{array}{c}\mathbf{8 8 1 6} \\
\text { HCND }\end{array}$ & $\begin{array}{c}\text { EXT-RAD } \\
\text { Ring }\end{array}$ & $\begin{array}{c}\text { HSD } \\
\text { Wrist/Ankle }\end{array}$ \\
\hline $\mathrm{H}_{\mathrm{p}}(0.07)$ & $10^{(\mathrm{a})}$ & $10^{(\mathrm{a})}$ & $\mathrm{n} / \mathrm{a}$ & 10 & $10^{(\mathrm{a})}$ \\
\hline $\mathrm{H}_{\mathrm{p}}(3)$ & 10 & 10 & $\mathrm{n} / \mathrm{a}$ & $\mathrm{n} / \mathrm{a}$ & $10^{(\mathrm{c})}$ \\
\hline $\mathrm{H}_{\mathrm{p}}(10)_{\gamma}$ & 10 & 10 & $\mathrm{n} / \mathrm{a}$ & $\mathrm{n} / \mathrm{a}$ & $10^{(\mathrm{c})}$ \\
\hline $\mathrm{H}_{\mathrm{p}}(10)_{\eta}$ & 20 & $\mathrm{n} / \mathrm{a}$ & $1-10^{(\mathrm{b})}$ & $\mathrm{n} / \mathrm{a}$ & 20 \\
\hline
\end{tabular}

(a) 50 mrem for pure beta radiation

(b) Minimum reported dose varies with neutron energy and corresponds to a net neutron signal on TLD $600=10 \mathrm{mR}$ equivalent.

(c) These results are calculated by ED and reported to REX but not used by REX in determining the extremity dose of record.

\subsection{Dosimeter Exchange and Selection of Frequency}

There are five categories of dosimeter assignment at Hanford:

- temporary

- monthly

- quarterly

- semi annually

- annually.

Hanford contractor dosimetry organizations are responsible for determining the dosimeter exchange frequency for each assigned dosimeter. The basis for the assignment is primarily the anticipated dose to be received.

When determining a dosimeter exchange frequency, consideration should be given to the potential for unreported dose as a result of the reporting thresholds described above (Table 6.2). For example, assignment of a monthly dosimeter with a 10 mrem reporting threshold to an individual who receives slightly less than 10 mrem each month could (theoretically) result in 0 mrem reported dose for the year when in fact the actual dose was nearly $120 \mathrm{mrem}$. This individual might be better served by a quarterly or annual dosimeter. However, statistically speaking, in this example the most probable reported dose for the year will be $58 \%$ of the true dose or about $70 \mathrm{mrem}$. The probability of the total reported dose being less than 20 mrem (i.e. unreported dose exceeding 100 mrem) for 12 dosimeters that actually received $10 \mathrm{mrem}$, is less than $0.5 \%$. For a very large number of dosimeters receiving exactly $10 \mathrm{mrem}$ and being processed with a reporting threshold of $10 \mathrm{mrem}$, the total reported dose will be very close to $58 \%$ of the true dose. This analysis is based on an assumed normal distribution of calculated dose results with a mean of 10 mrem and a standard deviation of 2 
mrem for dosimeters that were exposed to exactly 10 mrem. ${ }^{\text {(a) }}$ This assumption is a conservative approximation for monthly dosimeters processed with the current dosimetry system at Hanford.

When determining a dosimeter exchange frequency, consideration should also be given to minimizing uncertainty in calendar year recorded dose to the extent practicable. For example, the uncertainty in a single 8816 neutron dosimeter result reported for a PFP worker is approximately $\pm 40 \%$ and does not vary substantially with dose level as long as the dose is well above background. If the dosimeter is an annual dosimeter, then the uncertainty in calendar year recorded dose is $\pm 40 \%$. However, if monitoring for the calendar year is divided approximately equally among four quarterly dosimeters, the uncertainty in the recorded calendar year total will be approximately $\pm 20 \%$. A person receiving significant neutron dose might be better served with four quarterly dosimeters than a single annual dosimeter.

In addition to the routine exchange, consideration should be given to exchanging dosimeters when any of the following is suspected of having occurred:

- Damaged dosimeter (Mylar window, red tinted bar code window, external case)

- Breach of holder integrity (e.g. card falls out of holder or is removed from holder)

- Contamination of dosimeter (evaluate possible beta reading from contamination on Mylar window and subtract from dose result)

- Exposure of dosimeter to x-rays or dose from medical isotopes.

- Improper orientation of dosimeter during high dose work.

- Loss of control of dosimeter by user (e.g. dosimeter found on ground in parking lot).

- Unplanned use of dosimeter in radiation fields requiring special calibration factors. (e.g. work with pure beta emitters $<250 \mathrm{keV}$ average)

- Exposure of dosimeter to excessive temperatures (e.g. above $50^{\circ} \mathrm{C}$ )

\subsection{Dosimeter Wear Practices}

Each contractor is responsible for implementing dosimetry wear practices that enable accurate dosimetry results. The guidance on dosimeter wear practices in Section 6.7 of this manual is provided, along with a supporting technical basis, as one acceptable method of satisfying this objective. Alternative practices may

(a) D. J. Bates, "Description of Methods Used for Reporting Limit Effects", IOM to B. A. Rathbone, June 28, 2004, HEDP file. 
be implemented by each contractor when appropriate for their particular facilities.

The HSD and HCND are used to measure $H_{p}(0.07), H_{p}(3), H_{p}(10)_{\gamma}$ and $H_{p}(10)_{\eta}$. When used in uniform radiation fields these dosimeters should be worn on the front of the torso, between the neck and waist to obtain the most accurate response. (See Sections 6.7.1 and 6.7.2 below for guidance on use with anti-C protective clothing and lead aprons respectively.)

If a work task requires orientation of the individual with their back to the source for the majority of their exposure, then the HSD or HCND should be relocated to the back, or two dosimeters used (one front and one back). The human torso has a significant attenuating effect on both photon and neutron radiation. During those times when the worker is facing away from a source, a dosimeter on the front of the torso will be measuring significantly less $\mathrm{H}_{\mathrm{p}}(10)_{\gamma}$ and $\mathrm{H}_{\mathrm{p}}(10)_{\eta}$ than the actual dose received by those parts of the body facing the source. ${ }^{(a)(b)}$ Table 6.3 shows the dosimeter response on phantom when the phantom is facing away from the source (at 180 degrees). For the ${ }^{252} \mathrm{Cf}$ sources in Table 6.3, the dosimeter response shown is the response to the neutron component of the $\eta-\gamma$ radiation field produced by these sources.

When in neutron radiation fields, the HSD or HCND should be worn within 1.27 cm (1/2 inch) of the body (or protective vest) to the extent practicable. Studies using a bare ${ }^{252} \mathrm{Cf}$ neutron source have shown a significant decrease in the $\mathrm{H}_{\mathrm{p}}(10)_{\eta}$ response of the HCND when the dosimeter is located more than $1.27 \mathrm{~cm}$ from the surface of a phantom (see Chapter 5). In general, a significant decrease in response with increasing distance from the body or phantom is observed with most albedo neutron TLD designs (Piesch, Burgkhardt and Venkataraman 1982, Piesch and Burgkhardt 1985).

It is also important to understand the dependency of the HCND's $\mathrm{H}_{\mathrm{p}}(10)_{\eta}$ response on the composition and geometry of the backscattering material. Based on data obtained from phantom irradiations, the HCND requires a large mass of hydrogenous backscatter medium (e.g. $5-10 \mathrm{~kg}$ ) and the dosimeter must be at least $10 \mathrm{~cm}$ from the edge of the medium before the dosimeter's response becomes adequate. Inadequate backscatter is a particular concern if attempts are made to measure the neutron dose by placing the HCND on parts of the body other than the torso (e.g., arms, legs, head, etc.). When neutron dosimeters are worn on body parts other than the torso, allowances and/or adjustments should be made for the fact that the measured $\mathrm{H}_{\mathrm{p}}(10)_{\eta}$ may underestimate the true $\mathrm{H}_{\mathrm{p}}(10)_{\eta}$.

(a) B. A. Rathbone, "Impact of Phantom Size and Orientation on 8816 Neutron TLD Response" September 6, 2001, Letter to HEDP file.

(b) J.J. Fix, E.S. Gilbert and W.V. Baumgartner, "An Assessment of Bias and Uncertainty in Recorded Dose from External Sources of Radiation for Workers at the Hanford Site" PNL-10066, August, 1994 
Table 6.3 HSD/HCND Deep Dose Response on a Phantom Oriented $180^{\circ}$ from Source

\begin{tabular}{||c|c|c|c|c||}
\hline Source & $\begin{array}{c}\text { Radiation } \\
\text { Type }\end{array}$ & $\begin{array}{c}\text { Average } \\
\text { Energy } \\
(\mathrm{keV})\end{array}$ & $\begin{array}{c}\mathrm{H}_{\mathrm{p}}(10)_{\gamma} \\
\text { Response } \\
\text { Relative to } \\
\text { 0 degrees }\end{array}$ & $\begin{array}{c}\mathrm{H}_{\mathrm{p}}(10)_{\eta} \\
\text { Response } \\
\text { Relative to } \\
\text { 0 degrees }\end{array}$ \\
\hline \hline $\mathrm{M} 150$ & X-ray & 70 & 0.11 & \\
\hline $\mathrm{H} 150$ & X-ray & 117 & 0.16 & \\
\hline${ }^{137} \mathrm{Cs}$ & gamma & 662 & 0.36 & \\
\hline${ }^{252} \mathrm{Cf}$ mod & $\begin{array}{c}\text { gamma- } \\
\text { neutron }\end{array}$ & 550 & & 0.03 \\
\hline${ }^{252} \mathrm{Cf}$ bare & $\begin{array}{c}\text { gamma- } \\
\text { neutron }\end{array}$ & 2130 & & 0.15 \\
\hline
\end{tabular}

Extremity dosimeters should be worn in a manner to maximize the recorded dose. For example, when holding radioactive materials such as sample containers or contaminated objects in the hands, the ring dosimeter generally should be worn facing the palm of the hand. When holding non-radioactive objects such as tongs, tools or survey instruments, the ring dosimeter generally should be worn facing away from the palm of the hand. Because of the wide variety of possible circumstances, there is no single orientation appropriate for all jobs.

\subsubsection{Dosimeter Use with Protective Clothing}

When the radiation field is primarily penetrating radiation, whole body dosimeters may be worn either under the protective clothing or outside the protective clothing. When a substantial non-penetrating component (e.g. beta radiation, or photon radiation $<20 \mathrm{keV}$ average) is likely to be present, and the eyes or substantial areas of skin are unprotected (e.g., the face and neck), then the dosimeter should be placed on the outside of the protective clothing to ensure proper measurement of $\mathrm{H}_{\mathrm{p}}(0.07)$ and $\mathrm{H}_{\mathrm{p}}(3)$. When wearing whole body dosimetry outside protective clothing, it will be necessary to ensure that the dosimeter does not become contaminated. This may be accomplished in a manner that preserves the shallow dose response of the dosimeter by placing the dosimeter in a thin plastic bag with a wall density thickness $<10 \mathrm{mg} / \mathrm{cm}^{2}$. The standard $3 \times 7$ inch Whirl-Pak ${ }^{\circledR}$ bag has a density thickness of $5.4 \mathrm{mg} / \mathrm{cm}^{2}$ and is suitable for this purpose. ${ }^{\text {(a) }}$

When a single whole body dosimeter must be used in conjunction with a bullet proof protective vest, it should be worn on the outside of the vest. For bullet proof protective vests, an HEDP dosimetrist should be consulted regarding the potential effects of vest composition on dosimeter response.

(a) Whirl-Pak is a registered trademark of NASCO International Inc. 
Beta Dose Attenuation by Protective Clothing

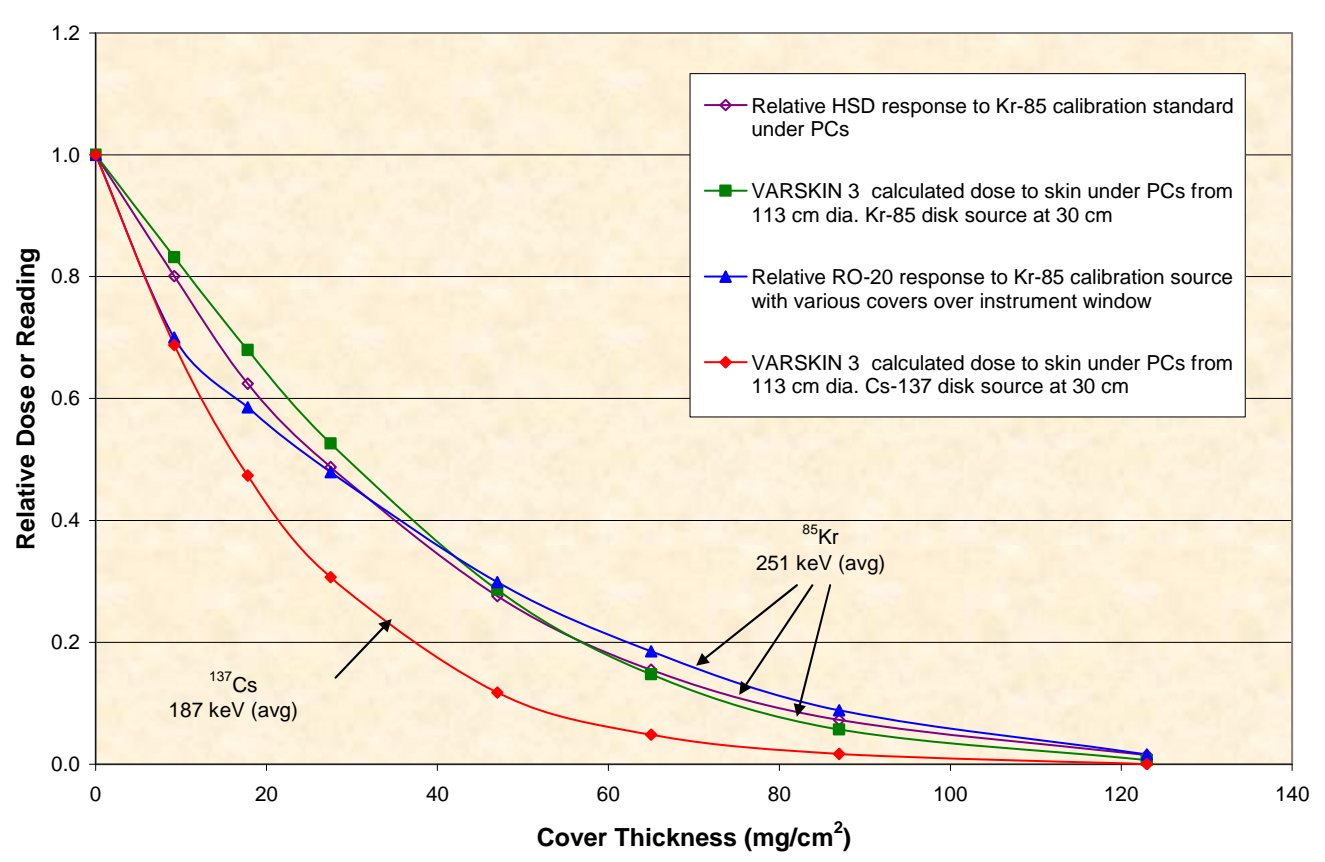

Figure 6.1 Measured Shallow Dose Attenuation by Protective Clothing for a ${ }^{85} \mathrm{Kr}$ Beta Source

When dosimeters are worn underneath protective clothing, the potential exists for significant reduction of the reported shallow dose. The degree of shallow dose attenuation by protective clothing depends on the types and energies of radiations involved. For work in neutron, photon, and energetic beta radiation fields, a single layer of protective clothing has minimal effect on the shallow dose response of the dosimeter. However, in intermediate and low energy beta fields (i.e. $<250 \mathrm{keV}$ average) even a single layer of protective clothing (typically 28 $\mathrm{mg} / \mathrm{cm}^{2}$ ) can significantly attenuate the shallow dose from beta radiation (Franklin and Gonzalez, 1997). Figure 6.1 shows the measured reduction in response of an RO-20 and an HSD to a ${ }^{85} \mathrm{Kr}$ beta calibration source at a distance of $30 \mathrm{~cm}$ when the dosimeter and instrument window are covered with various thicknesses of material simulating protective clothing. For comparison purposes, Figure 6.1 also shows the calculated reduction of shallow dose to skin under the protective clothing for ${ }^{85} \mathrm{Kr}$ and ${ }^{137} \mathrm{Cs}$ area sources ${ }^{(a)}$.

For beta particles sufficiently energetic to penetrate to the lens of the eye (i.e. $>$ $800 \mathrm{keV}$ ), a single layer of protective clothing covering the dosimeter will have minimal effect on the reported shallow dose but may have a measurable effect on the reported eye dose. The magnitude of this effect depends on the fraction of beta particles with a range at or near the depth of the lens of the eye (300

(a) B. A. Rathbone, "Impact of Protective Clothing on Dosimeter Shallow Dose Response in Mixed Fission Product BetaGamma Fields", March 21, 2006, HEDP File. 
$\left.\mathrm{mg} / \mathrm{cm}^{2}\right)$. For example the reduction of measured shallow dose from ${ }^{32} \mathrm{P}\left(\mathrm{E}_{\max }=\right.$ 1.7 MeV, $\mathrm{E}_{\mathrm{avg}}=700 \mathrm{keV}$ ) by one layer of protective clothing will be negligible whereas the reduction of measured eye dose may be as much as $20 \%-30 \%$ depending upon geometry. For ${ }^{90} \mathrm{Y}\left(\mathrm{E}_{\max }=2.28 \mathrm{MeV}, \mathrm{E}_{\text {avg }}=935 \mathrm{keV}\right)$ the reduction of measured shallow dose by one layer of protective clothing will be negligible whereas the reduction of measured eye dose may be as much as $6 \%$ $15 \%$ depending on geometry. This means that when working with beta emitters that are sufficiently energetic to allow placement of the dosimeter underneath protective clothing while retaining adequate shallow dose response, there is still the possibility for under estimating equivalent dose to the lens of the eye based on dosimeter reported eye dose if the lens of the eye is unprotected.

\subsubsection{Assessing the Potential for Under Recording Equivalent Dose to the Skin Using Knowledge of Average Beta Energy}

Radiation fields consisting of low energy beta particles (average spectral energy $<100 \mathrm{keV}$ ) and radiation fields consisting of mixtures of low energy beta particles with photons or neutrons generally do not present a significant potential for under estimating equivalent dose to unprotected areas of skin (e.g. face and neck) with a dosimeter worn under protective clothing because only a few percent of the beta particles can penetrate more than $30 \mathrm{~cm}$ of air to contribute dose to unprotected areas of skin.

Radiation fields consisting of neutrons, photons, and/or energetic beta particles (average spectral energy $>250 \mathrm{keV}$ ) generally do not present a significant potential for under estimating equivalent dose to unprotected areas of skin (e.g. face and neck) by a dosimeter placed under protective clothing because these radiations are only minimally attenuated by the protective clothing.

In general, the potential for significant shallow dose rates to unprotected areas of skin in conjunction with significant under estimation of equivalent dose to those areas by a dosimeter placed under protective clothing is greatest when the radiation field is produced entirely by surface activity consisting of intermediate energy beta emitters (between $100 \mathrm{keV}$ and $250 \mathrm{keV}$ average). This is true even for beta emitters that have associated gamma or x-ray emissions. The photon radiation associated with beta decay in surface activity accounts for a relatively small portion of the total shallow dose rate observed (typically less than 5\%).

Figure 6.2 shows the calculated attenuation of shallow dose from beta radiation by protective clothing of varying thickness for common beta emitters. The source geometry used in the calculations for this figure was a $1 \mathrm{~m}^{2}$ disk source of surface activity at a distance of $30 \mathrm{~cm}$ from the skin or dosimeter. ${ }^{\text {(a) }}$ Area sources are more likely to result in significant shallow dose attenuation and under estimation of equivalent dose to the skin than line or point sources. A single layer of typical cotton coverall has a density thickness of approximately 28 $\mathrm{mg} / \mathrm{cm}^{2}$.

(a) B. A. Rathbone, "Impact of Protective Clothing on Dosimeter Shallow Dose Response in Mixed Fission Product BetaGamma Fields", March 21, 2006, HEDP File. 
Beta Dose Attenuation in PCs for Selected Nuclides

( $30 \mathrm{~cm}$ from a $113 \mathrm{~cm}$ diameter disk source)

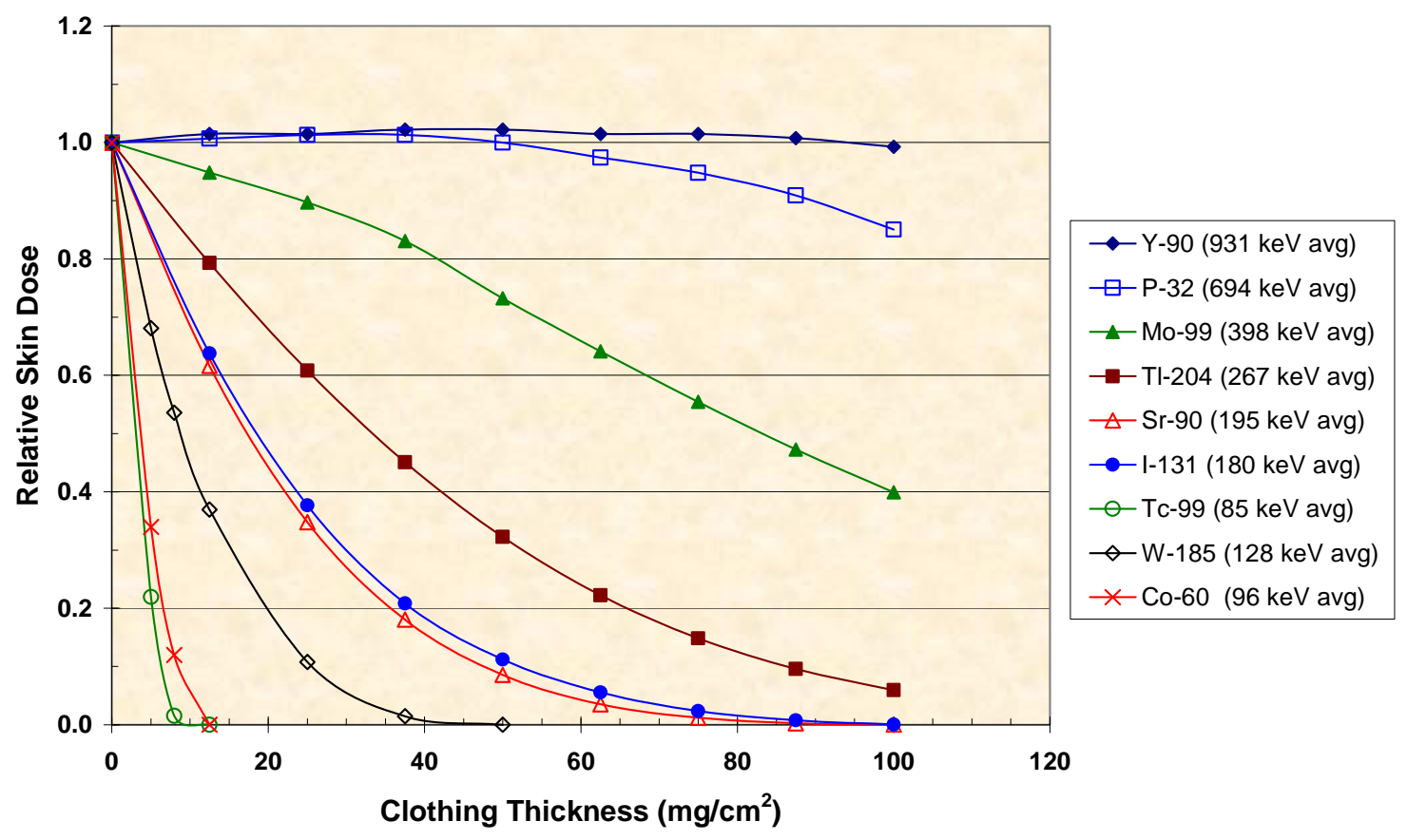

Figure 6.2 Beta Dose Attenuation for Common Beta Emitters

\subsubsection{Assessing the Potential for Under Recording Equivalent Dose to the Skin using Survey Instruments}

Survey instrument readings can be used to estimate the attenuating effect of protective clothing on dosimeter reported shallow dose and thus the potential for under estimating equivalent dose to unprotected areas of skin by a dosimeter worn under protective clothing. Measurements taken at a distance of $30 \mathrm{~cm}$ with an RO-3 or RO-20 that include a reading with an intermediate window thickness can be used in conjunction with Equation 6.1 to estimate the dose transmission factor (DTF).

$\mathrm{DTF}_{\mathrm{est}}=\mathrm{WO}_{\mathrm{PC}} / \mathrm{WO}$

(eqn. 6.1)

where:

$\mathrm{DTF}_{\text {est }}=$ estimated shallow dose transmission factor (dose under PC / dose outside PC)

WO $=$ open window reading with standard $7 \mathrm{mg} / \mathrm{cm}^{2}$ Mylar window

$\mathrm{WO}_{\mathrm{PC}}=$ open window reading with additional window material equal to the thickness of the protective clothing being worn 


\subsubsection{Exposure to Mixed Fission Product Activity in Sample Containers}

For work involving grab sampling and laboratory analysis of mixed fission products (MFP) where the majority of activity is contained inside a sample container, the potential for a dosimeter placed underneath protective clothing to significantly underestimate equivalent dose to unprotected areas of skin is small. The shielding afforded by the walls of sample containers such as glass jars, vials and centrifuge cones reduces this potential in two ways: 1) the reduction of beta dose rate from the container increases the relative contribution of any gamma emitters present in the sample to the shallow dose rate at the surface of the protective clothing and thus reduces the attenuating effect of the protective clothing on shallow dose to the dosimeter underneath, 2) with mixtures of hard and soft beta emitters, the container walls selectively attenuate the lower energy beta particles effectively hardening the beta spectrum and reducing the attenuating effect of the protective clothing on shallow dose to the dosimeter underneath. For activity consisting of ${ }^{90} \mathrm{Sr} /{ }^{90} \mathrm{Y}$ in secular equilibrium, a wall thickness of $100 \mathrm{mg} / \mathrm{cm}^{2}$ will remove nearly all of the ${ }^{90} \mathrm{Sr}$ beta particles, thus increasing the average energy of the beta spectrum significantly while reducing the beta dose rate by approximately $50 \%$. The resulting hard beta spectrum is not significantly attenuated by a single layer of protective clothing.

\subsubsection{Exposure to Mixed Fission Product Contaminated Surfaces}

The radionuclides most commonly associated with beta dose at Hanford are ${ }^{90} \mathrm{Sr}$, ${ }^{90} \mathrm{Y},{ }^{137} \mathrm{Cs}$ and ${ }^{137 \mathrm{~m}} \mathrm{Ba}$ in mixed fission product waste. These radionuclides account for more than $90 \%$ of the activity in Hanford waste tanks. The maximum and average beta energies for these radionuclides are shown in Table 6.4 below.

Table 6.4 Beta Energies for ${ }^{90} \mathrm{Sr},{ }^{90} \mathrm{Y}$ and ${ }^{137} \mathrm{Cs}$

\begin{tabular}{||c|c|c|c|c||}
\hline \hline Nuclide & $\begin{array}{c}\text { Decay mode } \\
\text { abundance }\end{array}$ & $\begin{array}{c}\text { Max energy } \\
(\mathrm{keV})\end{array}$ & $\begin{array}{c}\text { Avg energy } \\
(\mathrm{keV})\end{array}$ & $\begin{array}{c}\text { Range } \\
\left(\mathrm{mg} / \mathrm{cm}^{2}\right)\end{array}$ \\
\hline${ }^{90} \mathrm{Y}$ & 1.00 & 2283 & 935 & 1100 \\
\hline${ }^{90} \mathrm{Sr}$ & 1.00 & 546 & 196 & 185 \\
\hline${ }^{137} \mathrm{Cs}$ & 0.95 & 512 & 157 & 160 \\
\hline${ }^{137} \mathrm{Cs}$ & 0.05 & 1173 & 415 & 470 \\
\hline
\end{tabular}

Both ${ }^{90} \mathrm{Sr}$ and ${ }^{137} \mathrm{Cs}$ are beta emitters with average energies less than $250 \mathrm{keV}$ and produce beta radiation fields that will be attenuated by a single layer of protective clothing by more than $50 \%$. Figure 6.3 shows the calculated shallow dose attenuation in protective clothing for ${ }^{90} \mathrm{Sr},{ }^{90} \mathrm{Y},{ }^{137} \mathrm{Cs}$, and ${ }^{137 \mathrm{~m}} \mathrm{Ba}$ individually and in a 1:1:1:0.85 mixture. ${ }^{\text {(a) }}$ Figures 6.4 and 6.5 show the calculated shallow dose attenuation in protective clothing for other mixtures (identified by their Sr:Cs activity ratio). These figures are based on radiation fields produced entirely by surface activity which has not been covered or shielded in any way. Figure 6.6 provides a three dimensional projection of the information in Figures 6.4 and 6.5 .

(a) This corresponds to a Sr:Cs activity ratio of 1:1. In subsequent text and graphs where attenuation is expressed as a function of Sr:Cs activity ratio the underlying dose calculations assume that there is one ${ }^{90} \mathrm{Y}$ beta decay for every ${ }^{90} \mathrm{Sr}$ beta decay, and there are 0.85 ${ }^{137 \mathrm{~m}} \mathrm{Ba}$ photons released per ${ }^{137} \mathrm{Cs}$ beta decay. 


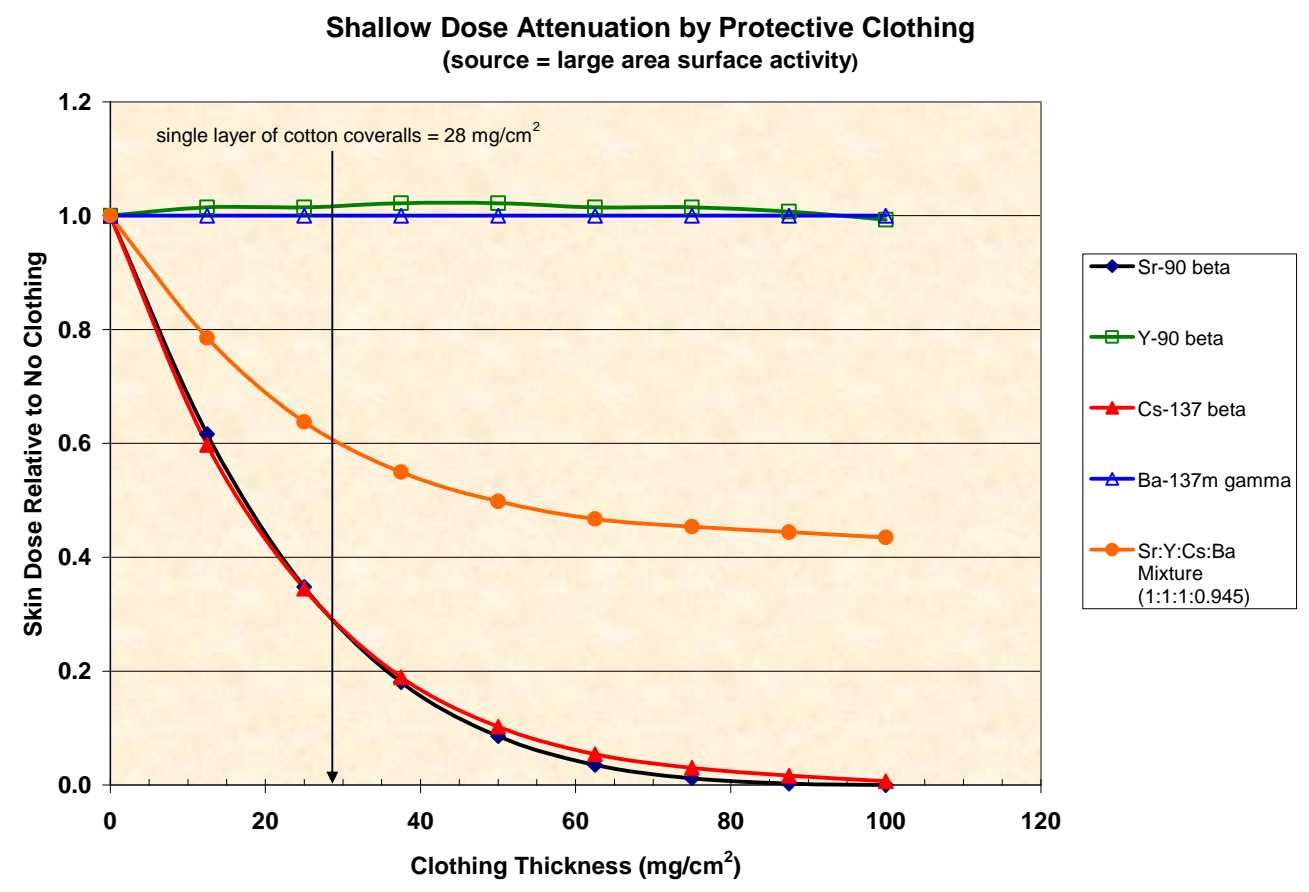

Figure 6.3 Calculated Shallow Dose Attenuation for ${ }^{90} \mathrm{Sr},{ }^{90} \mathrm{Y},{ }^{137} \mathrm{Cs}$ and ${ }^{137 \mathrm{~m}} \mathrm{Ba}$.

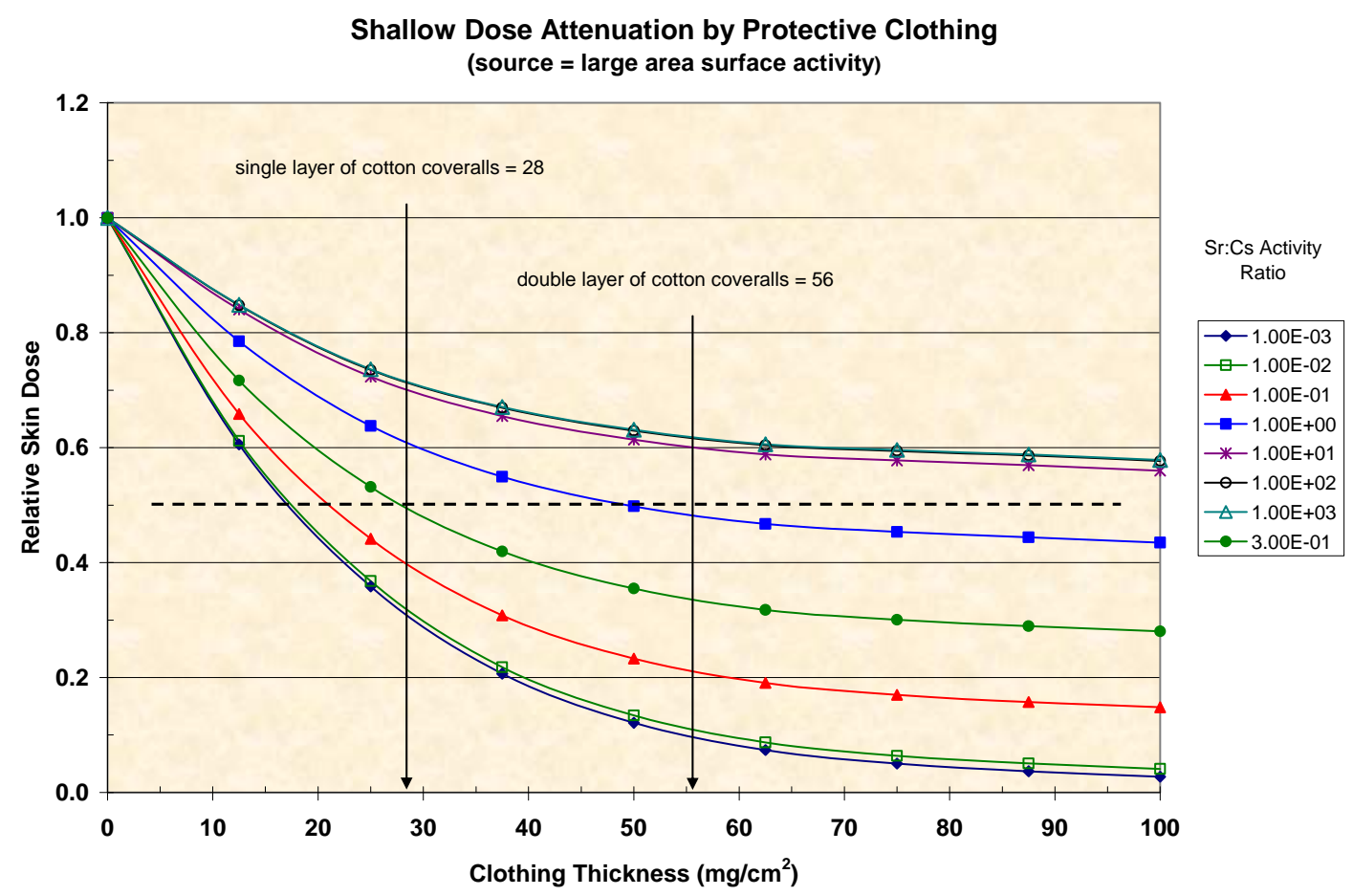

Figure 6.4 Calculated Shallow Dose Attenuation for Mixtures of ${ }^{90} \mathrm{Sr},{ }^{90} \mathrm{Y},{ }^{137} \mathrm{Cs}$ and ${ }^{137 \mathrm{~m}} \mathrm{Ba}$ as a Function of Clothing Thickness 


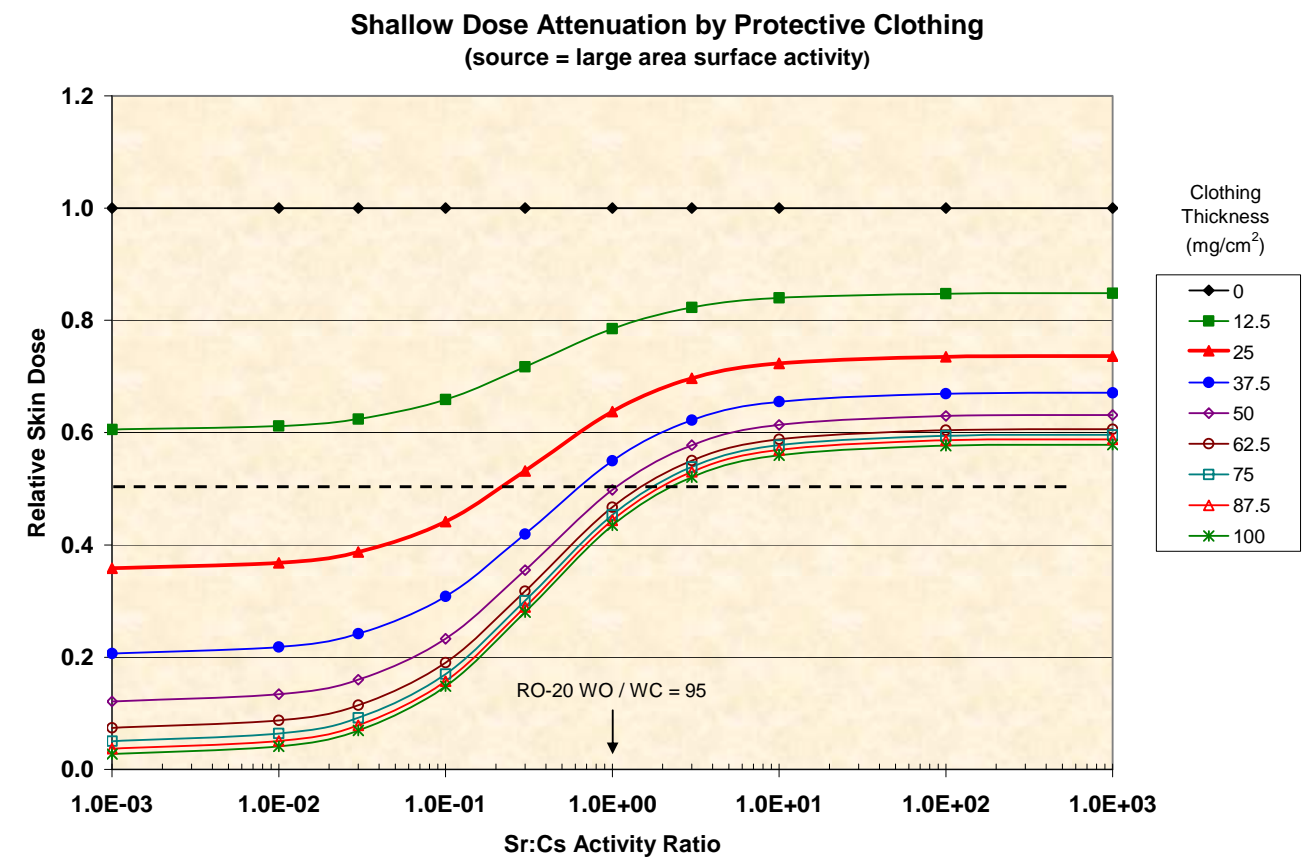

Figure 6.5 Calculated Shallow Dose Attenuation for Various Clothing Thicknesses as a Function of Sr:Cs Activity Ratio

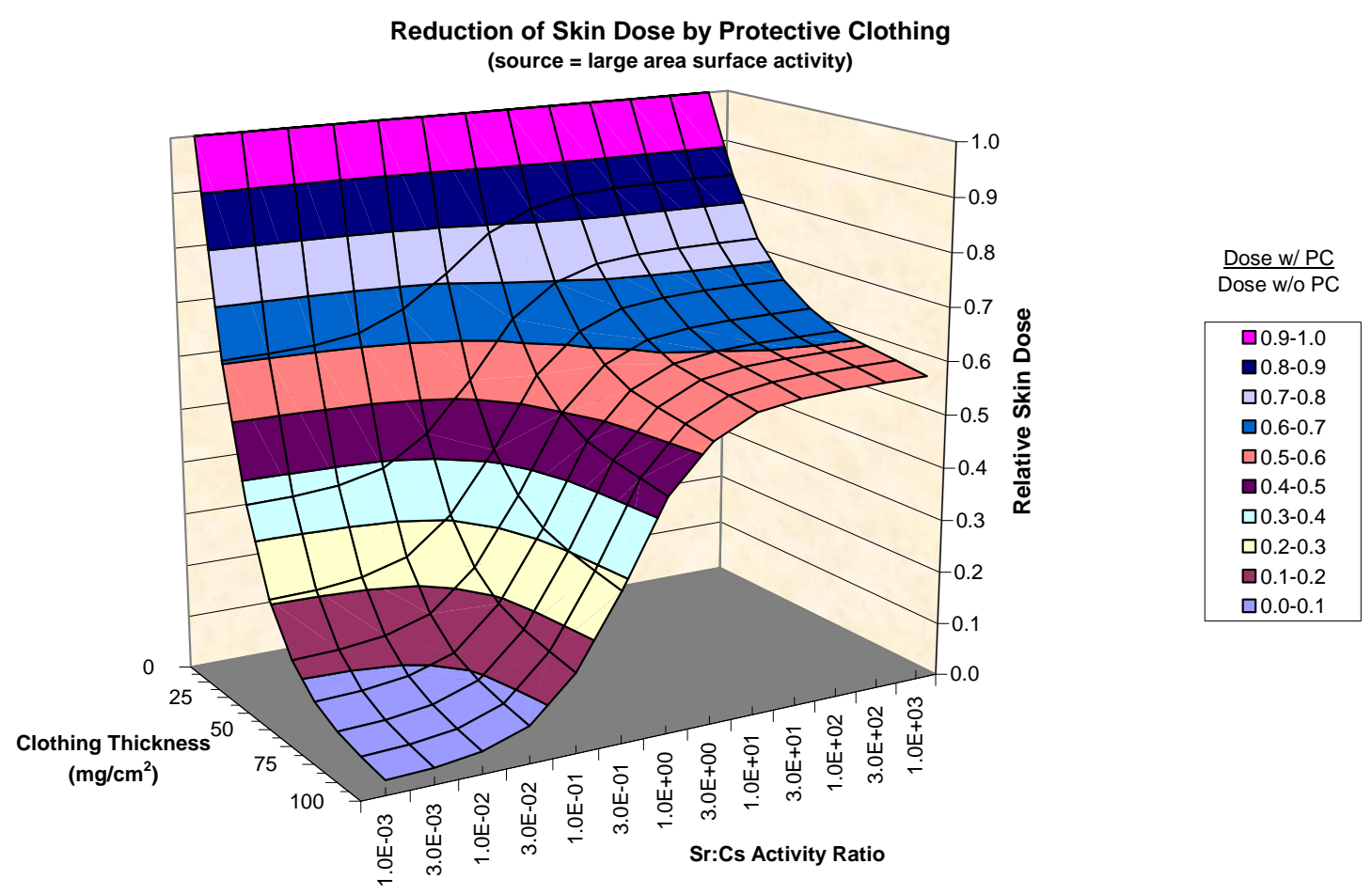

Figure 6.6 Shallow Dose Attenuation as a function of Clothing Thickness and Sr:Cs Activity Ratio 


\subsubsection{Dosimeter Use with Lead Aprons or Lead Vests in Uniform External Fields}

Lead aprons or vests are potentially useful ALARA tools. In terms of dose reduction to the body, lead aprons have a relatively small shielding effect for neutrons $(<10 \%$ reduction) but can be very effective for low energy photons. A lead apron with a rated effective thickness of $0.5 \mathrm{~mm}$ of $\mathrm{Pb} @ 85 \mathrm{kVp}$ results in essentially no reduction in $\mathrm{H}_{\mathrm{p}}(0.07), \mathrm{H}_{\mathrm{p}}(3)$ or $\mathrm{H}_{\mathrm{p}}(10)_{\gamma}$ for $662 \mathrm{keV}$ photons, but an approximate 10 fold reduction for $59 \mathrm{keV}$ photons, and greater than a 100 fold reduction for $17 \mathrm{keV}$ photons. In some Hanford environments, photons $<100$ $\mathrm{keV}$ have been shown to contribute a large enough fraction of the photon dose to make lead aprons worth consideration. At PFP locations where plutonium was sometimes stored in thin sealed steel cans, an apparent reduction factor between 2.5 and 4.0 for $\mathrm{H}_{\mathrm{p}}(10)_{\gamma}$ was measured under one $0.5 \mathrm{~mm}$ layer of lead apron. The $\mathrm{H}_{\mathrm{p}}(0.07), \mathrm{H}_{\mathrm{p}}(3)$ and $\mathrm{H}_{\mathrm{p}}(10)_{\gamma}$ response of HSD and HCND under a lead apron are considered to be relatively accurate and representative of the dose received by portions of the body under the apron. However, when worn on top of a lead apron, the response of these dosimeters may be affected and their results may need to be corrected. Recommended procedures for using dosimeters with lead aprons and for correcting the results have been developed and are available upon request. $^{\text {(a) }}$ HSD and $\mathrm{HCND} \mathrm{H}_{\mathrm{p}}(10)_{\eta}$ response is relatively unaffected whether the dosimeters are worn on top of or underneath the lead apron and does not need correction.

\subsection{When to Use Multiple Dosimeters for Work in Non-Uniform Fields}

The discussion of multiple dosimetry in this section, includes whole body and extremity dosimeters. Multiple dosimeters should be used when the personal dose equivalent received by a portion of the whole body or the extremities may significantly exceed the corresponding personal dose equivalent measured by the reference (i.e., chest) dosimeter. When the anticipated effective dose from external sources is significantly greater than the anticipated deep personal dose equivalent measured by the chest dosimeter, multiple whole body dosimeters should be worn. The need for assignment of multiple dosimeters is determined by the responsible contractor radiation control organization and should be documented in the applicable Radiation Work Permit.

Extremity dosimetry should be worn for specific jobs in non-uniform fields with large dose gradients in which it is anticipated that the extremities may receive a shallow personal dose equivalent more than 10 times the deep personal dose equivalent from photons and neutrons measured by the chest dosimeter and the extremities may receive a shallow personal dose equivalent greater than 500 mrem. In other words, extremity dosimeters should be worn when it is anticipated that:

$$
\begin{gathered}
\mathrm{H}_{\mathrm{p}}(0.07)_{\text {extremity }}>10 \times\left[\mathrm{H}_{\mathrm{p}}(10)_{\gamma}+\mathrm{H}_{\mathrm{p}}(10)_{\eta}\right]_{\text {chest }} \\
\underline{\text { and }} \\
\mathrm{H}_{\mathrm{p}}(0.07)_{\text {extremity }}>500 \mathrm{mrem}
\end{gathered}
$$

(a) B. A. Rathbone, "Recommendations on the Use of HCNDs with Lead Aprons at PFP" September 30, 2003, Letter to R. L. Hill (in HEDP files). 
The rationale for the ratio of 10 is the fact that the 10 CFR 835 limits for equivalent dose to the extremities and equivalent dose to the whole body differ by a factor of 10. By ensuring compliance with the whole body limit, compliance with the extremity limit will be ensured by adherence to the above criteria. Extremity dosimetry should also be considered for jobs with large dose gradients and variable exposure geometries resulting in unpredictable dose rates. The above criteria should be considered minimum guidelines. They do not preclude the use of extremity dosimetry under any circumstances where sound health physics judgment would warrant their use.

Eye dosimetry should be worn near the eyes for a specific job when it is anticipated that the eye personal dose equivalent received by the lens of the eye may exceed the deep personal dose equivalent measured by the chest dosimeter by $300 \%$ and also exceed $100 \mathrm{mrem}$. In other words, eye dosimetry should be used when it is anticipated that:

$$
\begin{gathered}
\mathrm{H}_{\mathrm{p}}(3)_{\text {eye }}>3 \times\left[\mathrm{H}_{\mathrm{p}}(10)_{\gamma}+\mathrm{H}_{\mathrm{p}}(10)_{\eta}\right]_{\text {chest }} \\
\text { and } \\
\mathrm{H}_{\mathrm{p}}(3)_{\text {eye }}>100 \mathrm{mrem}
\end{gathered}
$$

This guidance is based on the fact the limit for equivalent dose to the lens of the eye in 10 CFR 835 is a factor of 3 greater than the limit for equivalent dose to the whole body.

Multiple whole body dosimetry should be worn when either of the following two criteria are met.

1. The effective dose from external sources is expected to exceed the deep personal dose equivalent from photons and neutrons measured by the reference dosimeter by more than $30 \%$, and is expected to exceed 100 mrem.

2. The effective dose from external sources is expected to exceed the deep personal dose equivalent from photons and neutrons measured by reference dosimeter by more than $100 \mathrm{mrem}$.

In other words, multiple whole body dosimetry should be worn when it is anticipated that:

$$
\begin{gathered}
\mathrm{E}_{\text {external }}>1.3 \times\left[\mathrm{H}_{\mathrm{p}}(10)_{\gamma}+\mathrm{H}_{\mathrm{p}}(10)_{\eta}\right]_{\text {chest }} \text { and } \mathrm{E}_{\text {external }}>100 \mathrm{mrem} \\
\text { or } \\
\mathrm{E}_{\text {external }}>\left[\mathrm{H}_{\mathrm{p}}(10)_{\gamma}+\mathrm{H}_{\mathrm{p}}(10)_{\eta}\right]_{\text {chest }}+100 \mathrm{mrem}
\end{gathered}
$$

The above criteria may be considered as an acceptable alternative to the criteria in Article 512.4 of DOE-STD 1098-2008 DOE Standard - Radiological Control (DOE 2008b). The above criteria do not preclude the use of multiple dosimetry when deemed appropriate (e.g., because of uncertainties in worker movement or 
radiation field strength). The $30 \%$ difference criteria was chosen based on the approximate percentages by which an actual effective dose could exceed a chest dosimeter result under worst-case conditions, before multi-badging would be required under current guidance in Article 512.4.

Guidance on when to multi-badge is normally applied to a particular job episode, typically lasting not more than one month. For jobs that exceed one month in duration and involve multiple whole body dosimetry, the dosimetry should be processed at the end of each calendar month. For jobs that exceed one month in duration and involve routine chest and extremity dosimetry only (i.e., not a multipack), the dosimetry may be worn until the end of the calendar quarter before processing, if appropriate (i.e., if doses are expected to be low).

\subsubsection{Monitoring Workers with Multiple Whole Body Dosimeters}

Multiple whole body dosimeters should be issued as a packet for each individual. The packet must include a temporary chest dosimeter to replace the routine chest dosimeter as the person's primary (reference) dosimeter. Records must be maintained of the actual placement location for each dosimeter. Codes have been prepared for use by the contractor dosimetry organizations to identify the location of the respective multiple dosimeters as shown in Table 6.5.

If the individual's routine chest dosimeter is believed to have significant dose (e.g., greater than 100 mrem) or the individual's year-to-date dose is near an administrative control level (ACL), then it should be processed before or in conjunction with the multiple dosimeter packet to establish the individual's current dose status at the end of the job episode. After completion of the job, a new chest dosimeter would then need to be issued to the individual to be used as the primary (reference) dosimeter until the end of the normal dosimeter exchange period. However, if the routine chest dosimeter is known to have low dose, then it may be temporarily stored during multipack use, and worn as the primary (reference) dosimeter at times when the multipack is not being used, (including the remainder of the dosimeter's exchange period after the routine job has ended).

The external dose of record as shown in REX status reports and screens is expressed in terms of protection quantities such as effective dose, total effective dose, equivalent dose to the lens of the eye, equivalent dose to the skin, equivalent dose to the extremities. These protection quantities are calculated from operational quantities $\mathrm{H}_{\mathrm{p}}(0.07), \mathrm{H}_{\mathrm{p}}(3), \mathrm{H}_{\mathrm{p}}(10)_{\gamma}$ and $\mathrm{H}_{\mathrm{p}}(10)_{\eta}$ measured by dosimeters and stored in REX using the general relationships shown in Chapter 4. 
Table 6.5. Multiple Dosimeter Location Codes

\begin{tabular}{|c|c|c|c|}
\hline Body Location & \multicolumn{2}{|c|}{ Code } & Description \\
\hline Hand & $\begin{array}{l}\text { left } \\
\text { right }\end{array}$ & $\begin{array}{l}=\mathrm{A} \\
=\mathrm{B}\end{array}$ & $\begin{array}{l}\text { The hand includes the area from below the wrist to the end of } \\
\text { the fingers. }\end{array}$ \\
\hline Eye & $\begin{array}{l}\text { left } \\
\text { right }\end{array}$ & $\begin{array}{l}=\mathrm{C} \\
=\mathrm{D}\end{array}$ & $\begin{array}{l}\text { The eye includes only the eye; the rest of the face is included } \\
\text { in the head. }\end{array}$ \\
\hline Head & & $=\mathrm{E}$ & $\begin{array}{l}\text { The head includes the complete head and the neck, both front } \\
\text { and back, except for the eyes. }\end{array}$ \\
\hline Abdomen & & $=\mathrm{F}$ & $\begin{array}{l}\text { The abdomen includes the frontal area below the bottom of the } \\
\text { rib cage and above the groin. }\end{array}$ \\
\hline Wrist & $\begin{array}{l}\text { left } \\
\text { right }\end{array}$ & $\begin{array}{l}=\mathrm{G} \\
=\mathrm{H}\end{array}$ & The wrist includes the wrist and lower arm below the elbow. \\
\hline Thigh & $\begin{array}{l}\text { left } \\
\text { right }\end{array}$ & $\begin{array}{l}=\mathrm{I} \\
=\mathrm{J}\end{array}$ & $\begin{array}{l}\text { The thigh includes the leg area below the groin and above the } \\
\text { knee. }\end{array}$ \\
\hline Knee & $\begin{array}{l}\text { left } \\
\text { right }\end{array}$ & $\begin{array}{l}=\mathrm{K} \\
=\mathrm{L}\end{array}$ & The knee includes only the knee area. \\
\hline Lower Leg & $\begin{array}{l}\text { left } \\
\text { right }\end{array}$ & $\begin{array}{l}=\mathrm{M} \\
=\mathrm{N}\end{array}$ & $\begin{array}{l}\text { The lower leg includes the leg area below the knee and above } \\
\text { the ankle. }\end{array}$ \\
\hline Foot & $\begin{array}{l}\text { left } \\
\text { right }\end{array}$ & $\begin{array}{l}=\mathrm{O} \\
=\mathrm{P}\end{array}$ & The foot includes the ankle and the foot to the end of the toes. \\
\hline Groin & & $=\mathrm{Q}$ & $\begin{array}{l}\text { The groin is the frontal area of the body at the junction of the } \\
\text { thighs and the trunk of the body. }\end{array}$ \\
\hline Chest & & $=\mathrm{R}$ & $\begin{array}{l}\text { The chest includes the frontal area below the neck and above } \\
\text { the bottom of the rib cage. However, if the primary dosimeter } \\
\text { is placed at the belt line or above and below the neck, it will be } \\
\text { considered as the chest. }\end{array}$ \\
\hline Back & & $=\mathrm{S}$ & $\begin{array}{l}\text { The back includes the area of the back of the body trunk below } \\
\text { the neck and above the thighs. }\end{array}$ \\
\hline Upper Arms & $\begin{array}{l}\text { left } \\
\text { right }\end{array}$ & $\begin{array}{l}=\mathrm{T} \\
=\mathrm{U}\end{array}$ & $\begin{array}{l}\text { The upper arm includes the elbow and the arm above the } \\
\text { elbow and below the shoulder. }\end{array}$ \\
\hline
\end{tabular}

The methodology used to evaluate the dose of record for multiple dosimetry is actually a methodology for determining the amounts of the basic shallow, eye, deep, and neutron operational quantities to be added to (or subtracted from) the individual's record where appropriate. When a dosimeter is processed as a supplemental dosimeter under REX note code 85 (supplemental dosimeternormal processing) or 86 , (supplemental dosimeter-special processing), its results are not automatically entered into the individual's record. When a dosimeter is processed under any note codes other than 85 or 86 , its results are automatically entered into the record unless a reject flag has been previously set for the dosimeter in REX. In those cases where REX has automatically entered the results into the individual's record, an evaluation of any needed changes to the record will require a detailed knowledge of how results were interpreted by REX. For this reason, the recommended method for processing multiple dosimetry is to submit all dosimeters (including chest dosimeter) under note code 85 or 86 . 
For the sake of simplicity, the following discussion assumes that all dosimeters in the multipack were processed under note code 85 or 86 and that chest dosimeter results have not been automatically entered into the record by REX.

A documented evaluation by the respective contractor dosimetry representative is necessary for assignment of dose from a multiple dosimetry packet, even if the dose assigned is zero.

\subsubsection{Calculation of Effective Dose E}

When multiple dosimeters in a multi-pack are worn, the effective dose from external sources of radiation is calculated based on the products of the dosimetermeasured operational quantities (or their differences) and whole body compartmentalization factors applicable to the dosimeter-wearing locations consistent with the recommendations included in the DOE Radiation Protection Programs Guide (DOE 2008a). The dosimeter results (operational quantities) used are shallow dose equivalent $\mathrm{H}_{\mathrm{p}}(0.07)$, deep photon dose equivalent $\left[\mathrm{H}_{\mathrm{p}}(10)_{\gamma}\right]$, and deep neutron dose equivalent $\left[\mathrm{H}_{\mathrm{p}}(10)_{\eta}\right]$. Recent changes in the 2007 revision to 10 CFR 835 (DOE 2007a) have a direct impact on the use of compartment weighting factors for instances where multiple dosimeters are worn in a potentially non-uniform irradiation field. Specifically, the tissues with explicit weighting factors and the factors themselves, as well as the explicit identification of tissues comprising the remainder organs and how they should weighted (i.e. on a mass basis), has changed. Since the ANSI/HPS N13.41 Criteria for Performing Multiple Dosimetry (HPS 1997) standard has not yet been updated to incorporate these changes in the regulations, Hanford's effective dose calculation methodology has been revised to incorporate these recent regulatory changes. The technical basis for the revised compartment factors presented in Table 6.6 is documented in a letter report ${ }^{\text {(a) }}$. The following table and equations describe how effective dose is assessed from a single multi-pack.

Table 6.6. Whole Body Compartment Weighting Factors

\begin{tabular}{||l|c|c|c||}
\hline \multicolumn{1}{||c|}{ Compartment } & $\begin{array}{c}\mathrm{CF}_{\gamma} \text { for } \\
\mathrm{H}_{\mathrm{p}}(10)_{\gamma} \\
\text { Dosimeter } \\
\text { Results }\end{array}$ & $\begin{array}{c}\mathrm{CF}_{\eta} \text { for } \\
\mathrm{H}_{\mathrm{p}}(10)_{\eta} \\
\text { Dosimeter } \\
\text { Results }\end{array}$ & $\begin{array}{c}\mathrm{CF}_{\beta} \text { for } \\
\mathrm{H}_{\mathrm{p}}(0.07)_{\beta} \\
\text { Dosimeter } \\
\text { Results }\end{array}$ \\
\hline \hline Head and Neck & 0.108 & 0.108 & 0.0012 \\
\hline Thorax, above the diaphragm & 0.307 & 0.307 & 0.0028 \\
\hline Abdomen, including pelvis & 0.561 & 0.561 & 0.0028 \\
\hline Upper Right Arm & 0.006 & 0.006 & 0.0008 \\
\hline Upper Left Arm & 0.006 & 0.006 & 0.0008 \\
\hline Right Thigh & 0.006 & 0.006 & 0.0008 \\
\hline Left Thigh & 0.006 & 0.006 & 0.0008 \\
\hline
\end{tabular}

a. $\mathrm{H}_{\mathrm{p}}(0.07)_{\beta}=\left[\mathrm{H}_{\mathrm{p}}(0.07)-\mathrm{H}_{\mathrm{p}}(10)_{\gamma}\right]$ The difference represents additional dose to the skin (primarily from beta particles) not accounted for in the $\mathrm{H}_{\mathrm{p}}(10)_{\gamma}$ and $\mathrm{H}_{\mathrm{p}}(10)_{\eta}$ doses.

(a) R. L. Hill, "Technical Basis for HPDAC Proposal On Multiple Dosimetry Effective Dose Methodology - for HPDAC Review and Approval 3/17/2009” HEDP File. 
The equation used to calculate the external effective dose from photons is as follows:

$\mathrm{E}_{\gamma} \quad=\sum\left[\mathrm{H}_{\mathrm{p}}(10)_{\gamma, \mathrm{c}} * \mathrm{CF}_{\gamma, \mathrm{c}}\right]$

Where:

$\mathrm{E}_{\gamma} \quad=$ external effective dose from photons

$\mathrm{H}_{\mathrm{p}}(10)_{\gamma, \mathrm{c}}=\mathrm{H}_{\mathrm{p}}(10)_{\gamma}$ measured for compartment, $\mathrm{c}$, of the body with the dosimeter(s)

$\mathrm{CF}_{\gamma, \mathrm{c}}=$ compartment factor for compartment, $\mathrm{c}$, from Table 6.6.

and summation is performed over all compartments $\mathrm{c}$.

The equation used to calculate the external effective dose from neutrons is as follows:

$\mathrm{E}_{\eta} \quad=\Sigma\left[\mathrm{H}_{\mathrm{p}}(10)_{\eta, \mathrm{c}} * \mathrm{CF}_{\eta, \mathrm{c}}\right]$

Where:

$\mathrm{E}_{\eta} \quad=\quad$ external effective dose from neutrons

$\mathrm{H}_{\mathrm{p}}(10)_{\eta, \mathrm{c}}=\mathrm{H}_{\mathrm{p}}(10)_{\eta}(\mathrm{mrem})$ measured for compartment, $\mathrm{c}$, of the body with the dosimeter(s)

$\mathrm{CF}_{\eta, \mathrm{c}}=$ compartment factor for compartment, $\mathrm{c}$, from Table 6.6.

and summation is performed over all compartments c.

The equation used to calculate the external effective dose from beta particles and non-penetrating photons (i.e. dose to the skin that has not been accounted for in the calculation of $E_{\gamma}$ and $E_{\eta}$ above) is as follows:

$\mathrm{E}_{\beta} \quad=\sum\left[\mathrm{H}_{\mathrm{p}}(0.07)_{\beta, \mathrm{c}} * \mathrm{CF}_{\beta, \mathrm{c}}\right]$

Where:

$\mathrm{E}_{\beta} \quad=\quad$ external effective dose from beta particles and non-penetrating photons

$\mathrm{H}_{\mathrm{p}}(0.07)_{\beta, \mathrm{c}}=\left[\mathrm{H}_{\mathrm{p}}(0.07)_{\mathrm{c}}-\mathrm{H}_{\mathrm{p}}(10)_{\gamma, \mathrm{c}}\right]$ measured for compartment, $\mathrm{c}$, of the body with the dosimeter(s)

$\mathrm{CF}_{\beta, \mathrm{c}}=$ compartment weighting factor for compartment, $\mathrm{c}$, from Table 6.6.

and summation is performed over all compartments $\mathrm{c}$.

To determine the dose to a given compartment, the highest dosimeter result for that compartment is used. If the compartment was not monitored, the result for the nearest compartment monitored may be assigned.

The total external effective dose for a multi-pack would be the sum of the 
individual components calculated above, as follows:

$\mathrm{E} \quad=\mathrm{E}_{\gamma}+\mathrm{E}_{\eta}+\mathrm{E}_{\beta}$

\subsubsection{Assessment of Equivalent Dose to Skin, Lens of Eye, and Extremity from Multi-Pack Data}

When multiple whole body dosimetry is performed, equivalent dose to the skin $\left(\mathrm{H}_{\text {skin }}\right)$ and equivalent dose to the lens of the eye $\left(\mathrm{H}_{\text {eye }}\right)$ for the specific work activity monitored with the multi-pack should be assessed in addition to the total external effective dose $\left(\mathrm{E}_{\text {total }}\right)$. When assessing equivalent dose to the skin $\left(\mathrm{H}_{\text {skin }}\right)$ from multi-pack data, the highest $\left[\mathrm{H}_{\mathrm{p}}(0.07)+\mathrm{H}_{\mathrm{p}}(10)_{\eta}\right]$ result from a dosimeter worn in any whole body location should be used. When assessing equivalent dose to the lens of the eye, the highest $\left[\mathrm{H}_{\mathrm{p}}(3)+\mathrm{H}_{\mathrm{p}}(10)_{\eta}\right]$ result from a dosimeter worn in the head and neck compartment (or nearest monitored compartment) should be used. When assessing equivalent dose to the extremities, the highest $\left[\mathrm{H}_{\mathrm{p}}(0.07)+\mathrm{H}_{\mathrm{p}}(10)_{\eta}\right]$ result from a dosimeter worn in an extremity location should be used.

\subsubsection{Updating REX with Multi-Pack Dose Results}

The equations and methodology above describe how $\mathrm{E}, \mathrm{H}_{\text {eye }}, \mathrm{H}_{\text {skin }}$ and $\mathrm{H}_{\text {extremity }}$ would be assessed from multi-pack data for a single job or monitoring period. REX does not store values of $\mathrm{E}, \mathrm{H}_{\text {eye }}, \mathrm{H}_{\text {skin }}$ and $\mathrm{H}_{\text {extremity }}$ directly but rather calculates them for any given time period from the $\mathrm{H}_{\mathrm{p}}(0.07), \mathrm{H}_{\mathrm{p}}(3), \mathrm{H}_{\mathrm{p}}(10)_{\gamma}$, and $\mathrm{H}_{\mathrm{p}}(10)_{\eta}$ operational quantities reported by dosimeters during that time period (see Chapter 4). Therefore, the process of updating REX from multi-pack data is essentially an exercise in substituting values determined from the multi-pack data for the results that would normally have been entered from the chest dosimeter. For the purpose of updating REX (via the IODR process), the following process will be used:

- The assessed value of $\mathrm{E}_{\gamma}$ for the multi-pack should be entered as "deep" dose (in place of the $H_{p}(10)_{\gamma}$ value measured by the chest dosimeter).

- $\quad$ The assessed value of $E_{\eta}$ for the multi-pack should be entered as "neutron" dose (in place of the $\mathrm{H}_{\mathrm{p}}(10)_{\eta}$ value measured by the chest dosimeter).

- $\quad$ The assessed value of $\mathrm{H}_{\text {eye }}$ from the multi-pack should be entered as "eye" dose (in place of the $\mathrm{H}_{\mathrm{p}}(3)$ value measured by the chest dosimeter). NOTE: To avoid excessive conservatism in REX summation of quantities, it is acceptable to enter $\left[\mathrm{H}_{\text {eye }}-\mathrm{E}_{\eta}\right]$ as the "eye" dose.

- $\quad$ The assessed value of $\mathrm{H}_{\text {skin }}$ from the multi-pack should be entered as "shallow" dose (in place of the $\mathrm{H}_{\mathrm{p}}(0.07)$ value measured by the chest dosimeter). NOTE: To avoid excessive conservatism in REX summation of quantities, it is acceptable to enter $\left[\mathrm{H}_{\text {skin }}-\mathrm{E}_{\eta}\right]$ as the "shallow" dose.

- $\quad$ The assessed value of $\mathrm{H}_{\text {extremity }}$ from the multi-pack should be entered as "ring" dose (in place of the $\mathrm{H}_{\mathrm{p}}(0.07)$ value measured by the ring 
dosimeter). NOTE: To avoid excessive conservatism in REX summation of quantities, it is acceptable to enter the amount by which $\mathrm{H}_{\text {extremity }}$ exceeds $\mathrm{H}_{\text {skin }}$ as the "ring" dose (i.e. if $\mathrm{H}_{\text {extremity }}>\mathrm{H}_{\text {skin }}$ then ring dose $=\mathrm{H}_{\text {extremity }}-\mathrm{H}_{\text {skin }}$, otherwise ring dose $=0$ ).

It should be noted that this method of calculating effective dose and equivalent dose from multi-pack operational data may result in conservatism in the values for $\mathrm{E}, \mathrm{H}_{\text {eye }}, \mathrm{H}_{\text {skin }}$ or $\mathrm{H}_{\text {extremity }}$ that will be reported by $\mathrm{REX}$ on report cards or queries for any given time period, because of conservatism in the way in which REX sums operational quantities to obtain protection quantities for reporting to individuals and DOE. Refer to Chapter 4 of this manual for a description of how REX uses operational quantities. The DOE "Radiation Protection Programs Guide," (DOE 2008a) states that in addition to use of a compartment model, the highest equivalent dose reported at any body location may be reported as the effective dose. If this option were to be used, an even greater overestimate of the protection quantities would result.

\subsection{Personnel Dosimeter Accreditation}

During the 1980s, performance testing standards for personnel dosimeters were formally adopted by laboratory accreditation programs administered by the National Institute of Standards and Technology (NVLAP) and the U.S. Department of Energy (DOELAP). The performance tests involved both protection and accident level doses and several radiation types and mixtures. Accreditation involves a two-step process: the laboratory must first pass a performance test, and then pass a technical program appraisal. Upon successful completion of both steps, the laboratory is accredited. Hanford voluntarily participated in several of these tests during the early 1980s and received DOELAP accreditation for the basic and multipurpose dosimeter designs that were part of the previous Hanford TLD system. The first accreditation was granted, effective January 1, 1990, in all categories requested for testing. This TLD system's performance was successfully retested in 1991, leading to reaccreditation in 1993.

During 1994, 1996, 1998, 2000, 2002,2004, 2006, and 2008 DOELAP accreditation was applied for and granted for the HSD, and HCND that are part of the current Harshaw dosimetry system. Beginning in 2002, accreditation for the HCND with CR39 was discontinued. Beginning in 1998, the DOELAP accreditation process was expanded by DOE to include extremity dosimetry on a voluntary basis. (Accreditation for Extremity Dosimetry is expected to become mandatory by 2011.) Beginning with the spring 1998 DOELAP test session, Hanford submitted the HRD and HSD Wrist/Ankle Dosimeter (HSD worn on wrist or ankle) for testing and accreditation. DOELAP granted formal accreditation for these extremity dosimeters in November 1998. They were reaccredited in 2000, 2002, 2004, 2006, and 2008. In 2002, 2004, 2006, and 2008 the EXT-RAD extremity dosimeter was also submitted to DOELAP for performance testing and granted accreditation. Beginning with performance testing in 2010, the HRD will be discontinued at Hanford and only the EXTRAD Ring dosimeter and HSD Wrist/Ankle dosimeter will be tested and accredited as extremity dosimeters. 
NVLAP accreditation was also obtained in 1997, 2000, and 2002 for the HSD, HCND and EXT-RAD but has since been discontinued. No accreditation programs currently exist for environmental, area, or nuclear accident dosimetry.

\subsection{DOELAP Accreditation Categories}

The DOELAP categories selected for testing are based on radiation fields expected in Hanford work environments. For the testing performed during 2008 the categories selected for Hanford dosimeters are shown in Tables 6.7 and 6.8.

Hanford Standard Dosimeter (HSD). The DOELAP category IIIB for plutonium work environments was chosen for low-energy photons because of the large inventory of plutonium at Hanford. Category IIIA was chosen because of special radioactive materials and X-ray sources used in PNNL and other labs at Hanford. The general beta radiation category, consisting of ${ }^{90} \mathrm{Sr}$ or ${ }^{204} \mathrm{Tl}$ beta sources, was chosen because of the diversity of potential beta sources at Hanford, including the large quantities of ${ }^{90} \mathrm{Sr}$ material stored at the Hanford Waste Encapsulation Storage Facility and in waste tanks. Waste tank sampling and laboratory analysis activities can involve substantial shallow doses from ${ }^{90} \mathrm{Y}$ beta particles. Category VI (bare and moderated) was chosen for the HSD because it is used on a limited basis as a neutron dosimeter. Workers expected to receive less than $100 \mathrm{mrem} /$ year neutron dose may be monitored with an HSD.

Hanford Combination Neutron Dosimeter (HCND). All DOELAP categories requested for accreditation for the HSD were included for the HCND. In the neutron category, testing is performed with the moderated ${ }^{252} \mathrm{Cf}$ neutron spectrum in addition to the bare ${ }^{252} \mathrm{Cf}$ neutron spectrum because of the wide variety of neutron spectra in Hanford neutron work environments.

EXT-RAD Ring Dosimeter and HSD Wrist/Ankle Dosimeter The performance test categories shown in Table 6.8 were adopted from HPS N 13.32 (HPS 1996a) for use by DOELAP. Because the Hanford beta source term includes ${ }^{90} \mathrm{Sr} /{ }^{90} \mathrm{Y}$, and most extremity exposure at Hanford is from sources at a distance (i.e. non-contact geometry), Category IV-C was selected for performance testing for Hanford extremity dosimeters. Slab uranium was not chosen for performance testing because uranium handling activities and slab geometries currently do not represent a significant source of extremity exposure at Hanford. 
Table 6.7. Whole Body Dosimeter Test Categories

\begin{tabular}{|c|c|c|}
\hline \multirow{2}{*}{ Test Category } & \multicolumn{2}{|c|}{ Dosimeter Designations } \\
\hline & HSD & HCND \\
\hline $\begin{array}{l}\text { I. } \text { High-Dose }^{\mathbf{a}} \\
\text { Low-energy photons (M150) }\end{array}$ & $\mathrm{X}$ & $\mathrm{X}$ \\
\hline $\begin{array}{l}\text { II. High-Dose }{ }^{\mathbf{b}} \\
\text { High-energy photons }\left({ }^{137} \mathrm{Cs}\right)\end{array}$ & $\mathrm{X}$ & $\mathrm{X}$ \\
\hline III. Low energy photons & & \\
\hline $\begin{array}{l}\text { A. General } \\
\text { NIST filtered X-rays (M30, } \\
\text { S60, M150,H150) }\end{array}$ & $\mathrm{X}$ & $\mathrm{X}$ \\
\hline $\begin{array}{l}\text { B. Plutonium } \\
\text { Monoenergetic, } 15-20 \mathrm{keV} \\
\text { Monoenergetic, } 55-65 \mathrm{keV} \\
{ }^{241} \mathrm{Am}\end{array}$ & $\mathrm{X}$ & $\mathrm{X}$ \\
\hline IV. High-energy photons $\left({ }^{137} \mathrm{Cs}\right)$ & $\mathrm{X}$ & $\mathrm{X}$ \\
\hline V. Beta particles & & \\
\hline $\begin{array}{l}\text { A. General (Point Geometry) } \\
\left({ }^{90} \mathrm{Sr} /{ }^{90} \mathrm{Y},{ }^{204} \mathrm{Tl}\right)\end{array}$ & $\mathrm{X}$ & $\mathrm{X}$ \\
\hline B. Slab uranium & & \\
\hline $\begin{array}{l}\text { C. Special (Point Geometry) } \\
\left({ }^{\mathbf{c}} \mathrm{Sr} /{ }^{90} \mathrm{Y},{ }^{204} \mathrm{Tl}\right)\end{array}$ & & \\
\hline VI. Neutron ${ }^{d}$ & & \\
\hline${ }^{252} \mathrm{Cf}$ (bare) & $\mathrm{X}$ & $\mathrm{X}$ \\
\hline${ }^{252} \mathrm{Cf}$ (moderated) & $\mathrm{X}$ & $\mathrm{X}$ \\
\hline $\begin{array}{l}\text { VII. Mixtures (includes all } \\
\text { below) } \\
\text { III \& IV } \\
\text { III \& V } \\
\text { IV \& V } \\
\text { III \& VI } \\
\text { IV \& VI }\end{array}$ & $\mathrm{X}$ & $\mathrm{X}$ \\
\hline
\end{tabular}

a. Automatically entered into Category IIIA or IIIB

b. Automatically entered if entered into Category IV

c. Specify which beta source is selected for irradiation.

d. Specify which neutron source, or both sources, selected for irradiation. 
Table 6.8 Extremity Dosimeter Test Categories

\begin{tabular}{|c|c|c|}
\hline \multirow{2}{*}{ Test Category } & \multicolumn{2}{|c|}{ Dosimeter Designations } \\
\hline & EXT-RAD ${ }^{a}$ & HSD $^{b}$ \\
\hline \multicolumn{3}{|l|}{$\begin{array}{l}\text { I. High-Dose } \\
\text { A. Low-energy photons only } \\
\text { (M150) }\end{array}$} \\
\hline \multicolumn{3}{|l|}{$\begin{array}{l}\text { B. High-energy photons only } \\
\left({ }^{137} \mathrm{Cs}\right)\end{array}$} \\
\hline $\begin{array}{l}\text { B. General, low and high energy } \\
\text { photons }^{\mathrm{a}}\left(\mathrm{M} 150,{ }^{137} \mathrm{Cs}\right)\end{array}$ & $\mathrm{X}$ & $\mathrm{X}$ \\
\hline \multicolumn{3}{|l|}{$\begin{array}{l}\text { II. Low-energy photons } \\
\text { (NIST-filtered techniques) }\end{array}$} \\
\hline $\begin{array}{l}\text { A. } \text { General }^{\mathrm{c}} \text { (M30, M60, } \\
\text { M100, M150, H150) }\end{array}$ & $\mathrm{X}$ & $\mathrm{X}$ \\
\hline $\begin{array}{ll}\text { B. } & \text { High-energy }^{c} \\
& \text { (M100, M150, H150) }\end{array}$ & & \\
\hline $\begin{array}{l}\text { III. High-energy photons }{ }^{\mathbf{c}} \\
\qquad\left({ }^{137} \mathrm{Cs},{ }^{60} \mathrm{Co}\right)\end{array}$ & $\mathrm{X}$ & $\mathrm{X}$ \\
\hline \multicolumn{3}{|l|}{ IV. Beta particles } \\
\hline $\begin{array}{l}\text { A. Low-energy only } \\
\left({ }^{204} \mathrm{Tl}\right)\end{array}$ & & \\
\hline $\begin{array}{l}\text { B. High-energy only } \\
\left({ }^{90} \mathrm{Sr} /{ }^{90} \mathrm{Y}\right)\end{array}$ & & \\
\hline $\begin{array}{l}\text { C. } \quad \text { General }^{\mathrm{c}} \\
\left.\quad{ }^{90} \mathrm{Sr} /{ }^{90} \mathrm{Y},{ }^{204} \mathrm{Tl}\right)\end{array}$ & $X$ & $\mathrm{X}$ \\
\hline D. Slab uranium & & \\
\hline
\end{tabular}

a.EXT-RAD: Harshaw XD-740 chipstrate in EXT-RAD vinyl pouch and flexible ring strap

b. HSD: Hanford standard dosimeter used in wrist/ankle configuration

c. Each dosimeter will be irradiated with only one of the sources. 


\subsection{Radiation Types Not Covered by DOELAP Performance Testing}

Radiation types and energies not covered in the DOELAP performance testing standard, may require the use of facility specific correction factors and or facility specific algorithms. Examples of this for the HSD and HCND would be neutrons from accelerators or $(\alpha, n)$ sources, and beta particles from soft beta emitters with average beta energies less than approximately $250 \mathrm{keV}$ (the lowest energy source used in DOELAP performance testing). Correction factors for special radiation fields may be based on a knowledge of dosimeter response characteristics and a general knowledge of the radiation types and energies involved. However, if significant uncertainty exists, and significant exposure is expected, instrument measurements should be performed to validate the accuracy of the chosen correction factors. For photons, the LiF phosphors in Hanford dosimeters are nearly tissue equivalent. Because of this fact, and the fact that the DOELAP performance test standard is fairly comprehensive in scope for photons, the likelihood of facility specific corrections and or algorithms being needed for photon fields is relatively small.

\subsection{Facility Calibration Codes}

Facility calibration codes are sometimes necessary to overcome limitations in dosimeter technology. An awareness of dosimeter limitations and the proper use of facility calibration codes is essential for obtaining accurate dose of record (see Sections 6.3-6.7). A two-digit facility calibration code is used with both whole body and extremity dosimetry to identify calibration factors to be used by the dose algorithms (see Table 6.1). When a facility calibration code is not provided by the user, a default calibration code, 00 is used to calculate doses. At present, facility calibration codes are used to identify the neutron calibration factor to be applied to the HSD and the ring correction factor to be applied to the EXT-RAD. For the HSD, the default calibration code 00 results in a neutron calibration factor appropriate for unmoderated ${ }^{252} \mathrm{Cf}$ neutrons being applied. For the HSD, the calibration code also conveys whether neutron dose is expected or not. When neutrons are detected and none were expected the dose result is flagged as suspect. For the HSD, the default calibration code 00 is defined such that neutron dose is expected. For rings, the default calibration code 00 causes a ring correction factor of 2.0 to be applied to the dose results, which is appropriate for most extremity exposure received at Hanford including work at PFP. This factor adequately corrects for under response to beta radiation and, in the case of PFP, the lack of TLD 700 response to neutron radiation. Ring correction factors are discussed in greater detail in Chapter 5. The general use of facility calibration codes in dose calculation is discussed in greater detail in Chapter 5.

\subsection{Uncertainty in Recorded Dose}

Assessment of uncertainty for recorded Hanford dose has been the subject of three PNNL reports (Wilson et al. 1990; Fix, Gilbert, and Baumgartner 1994; Fix, Wilson, and Baumgartner 1996). These reports conclude that for historical dosimetry systems used until January 1, 1995, when the current state-of-the-art thermoluminescent (TL) dosimetry system was implemented, the dosimetry technology for photon (i.e., x-rays and gamma rays) and high-energy beta radiation was well developed with generally low uncertainties. However, with 
existing technology, the dosimetry for neutron and lower-energy beta radiation has considerably larger uncertainties. For neutron and beta radiation, accurate personnel dosimetry is dependent upon associated field instrument measurements and the use of field correction factors where appropriate. Radiation protection personnel need to be aware of the angular and energy dependence characteristics of Hanford dosimeters when assigning dosimeters, particularly with respect to beta and neutron radiation.

For the current dosimetry system good accuracy for personal dose equivalent is expected for any source of photon radiation. For beta-gamma dosimetry the uncertainty in personal dose equivalent is estimated to be about $\pm 30 \%$ for shallow dose equivalent, $\pm 15 \%$ for deep dose equivalent, and $\pm 15 \%$ for shallow dose equivalent to the extremities, when doses are well above natural background levels. ${ }^{\text {(a) }}$ For neutron dosimetry with the HCND, uncertainty is estimated to be about $\pm 40 \%{ }^{\text {(b) }}$ These are standard $(1 \sigma)$ uncertainties on individual dosimeter results estimated from the results of laboratory and field measurements. The respective uncertainties in the cumulative personal dose equivalent reported over a calendar year or lifetime may be less, depending on the number and magnitude of dosimeter results recorded. Characteristics of the current dosimetry system are described in greater detail in Chapter 5 of this manual.

\subsection{Approved Dosimeter Configurations}

The terms and conditions for DOELAP Accreditation include the stipulation that dosimeters are to be used in the field in the same configurations used for DOELAP Performance Testing or in configurations for which technical equivalency has been determined by DOELAP. Dosimeters are issued by the HEDP in these tested configurations. ${ }^{(c)}$ Limited use of dosimeters in configurations other than these, for specific field applications, may be acceptable, provided that the configurations are reviewed in advance by an HEDP Dosimetrist on a case by case basis to verify adequacy for the intended application. Routine use of dosimeters in configurations other than those tested by DOELAP must be evaluated and approved by DOELAP for Technical Equivalency before use. Dosimeter configurations tested by DOELAP and approved for general use at Hanford are shown in Figures 6.7 - 6.16. NOTE: The polyethylene foam pad shown inserted behind the 8816 dosimeter in Figure 6.10 and Figure 6.12 may not be present in all HCNDs. The pads have been inserted on an as needed basis to keep the 8816 dosimeter from sliding out of the HCND holder.

Minor variations in dosimeter configuration that have been determined to be

(a) B. A. Rathbone, "Analysis of Uncertainty in 8825 Dosimeter Results" November 17, 1999, letter to HEDP file. B. A. Rathbone, "95\% Confidence Intervals for 8825 Dosimeter Results" November 22, 1999, letter to HEDP file. S. E. Huneycutt, "Uncertainty Documentation for the 8816 TLD and CR-39 Track Etch Dosimeters" October 8, 1999, letter to HEDP file.

S. E. Huneycutt, "Re-evaluation of Measurement Uncertainty in XD740 Ring Dosimeter Results" March 30, 2000 letter to HEDP file.

(b) B. A. Rathbone, "Verification of 8816 Performance in PFP Neutron Fields" March 3, 2004, letter to HEDP file.

(c) PNADs are inserted into the HCND holder in the field by the user during dosimeter exchange. 
acceptable include the following:

- Placement of thin (i.e. less than $10 \mathrm{mg} / \mathrm{cm}^{2}$ ) plastic labels on the outside of the dosimeter, as long as they do not obstruct the beta window.

- Temporary placement of the dosimeter inside a thin (i.e. less than 10 $\mathrm{mg} / \mathrm{cm}^{2}$ ) plastic bag for the purpose of preventing contamination as shown in Figures 6.15 and 6.16.

Variations in dosimeter configuration not considered acceptable include the following:

- Permanent placement of a whole body dosimeter inside a vinyl pouch as shown in Figure 6.17. This configuration, which involves the addition of approximately $57 \mathrm{mg} / \mathrm{cm}^{2}$ of clear vinyl in front of two of the four elements in the dosimeter has been shown to have an unacceptable impact on the dosimeter's low energy x-ray response. ${ }^{\text {(a) }}$

- Permanent placement of tape, labels or other materials over the beta window of the HSD.

Potential problems with use of dosimeters in configurations different from those tested by DOELAP are described in a DOELAP Information Advisory (DOE, 2005). Clarification on what constitutes dosimeter configuration within the context of the DOELAP advisory is provided in a DOELAP Information Notice (DOE, 2006). In general, altering the configuration of a dosimeter means altering the physical construction such that the filtration in front of and/or behind the sensitive elements is changed. Orientation and placement of dosimeters on the body or protective clothing are considered wear practices as opposed to dosimeter configuration.

Impacts of protective clothing and protective vests on dosimeter response and recommended wear practices are discussed in Section 6.7 of this chapter.

(a) "Evaluation Of Impact Of Clear Vinyl Security Credential Holder On HSD Performance And Reported Doses At Hanford", Prepared April 10, 2006, by B. A. Rathbone, HEDP file. 


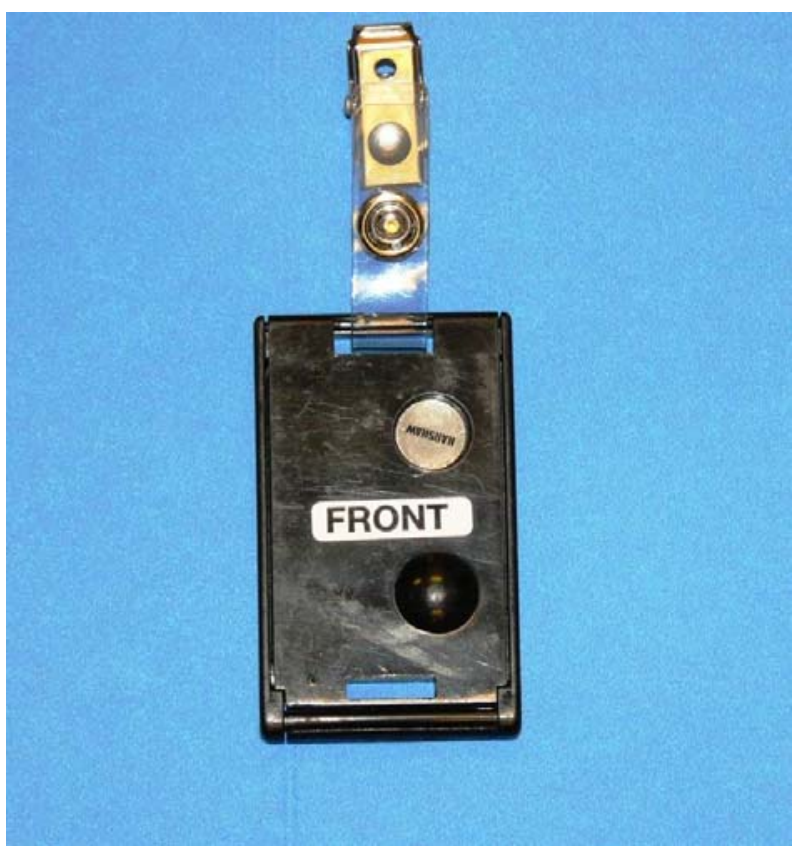

Figure 6.7 HSD Front Side

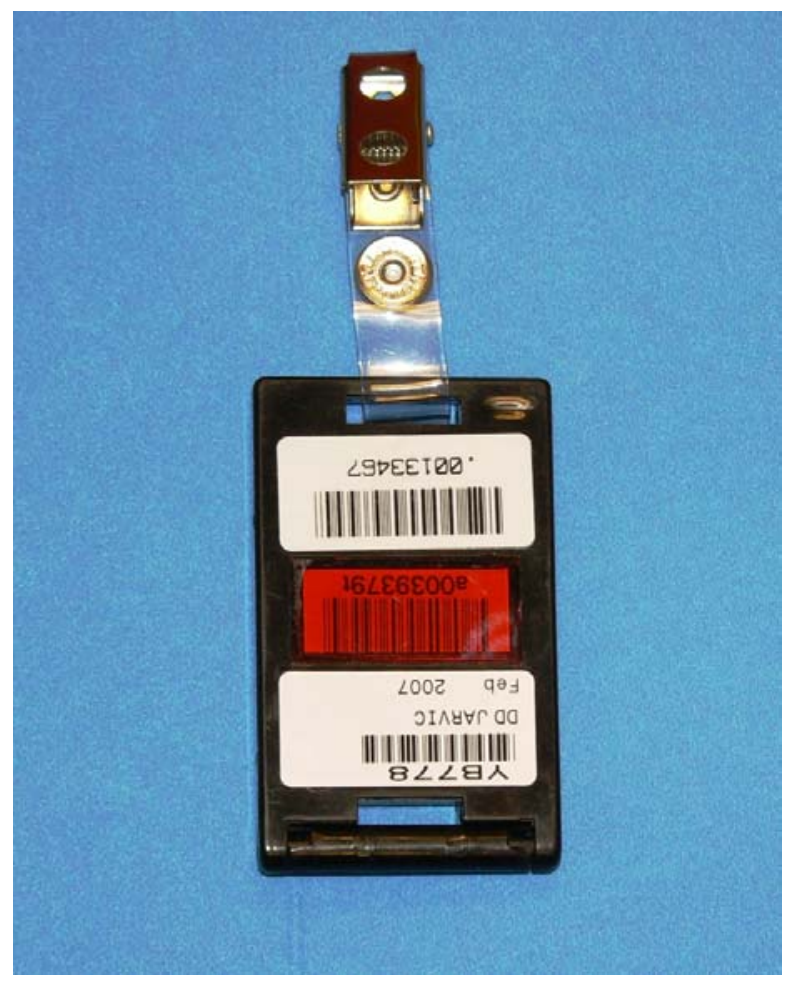

Figure 6.8 HSD Back Side 


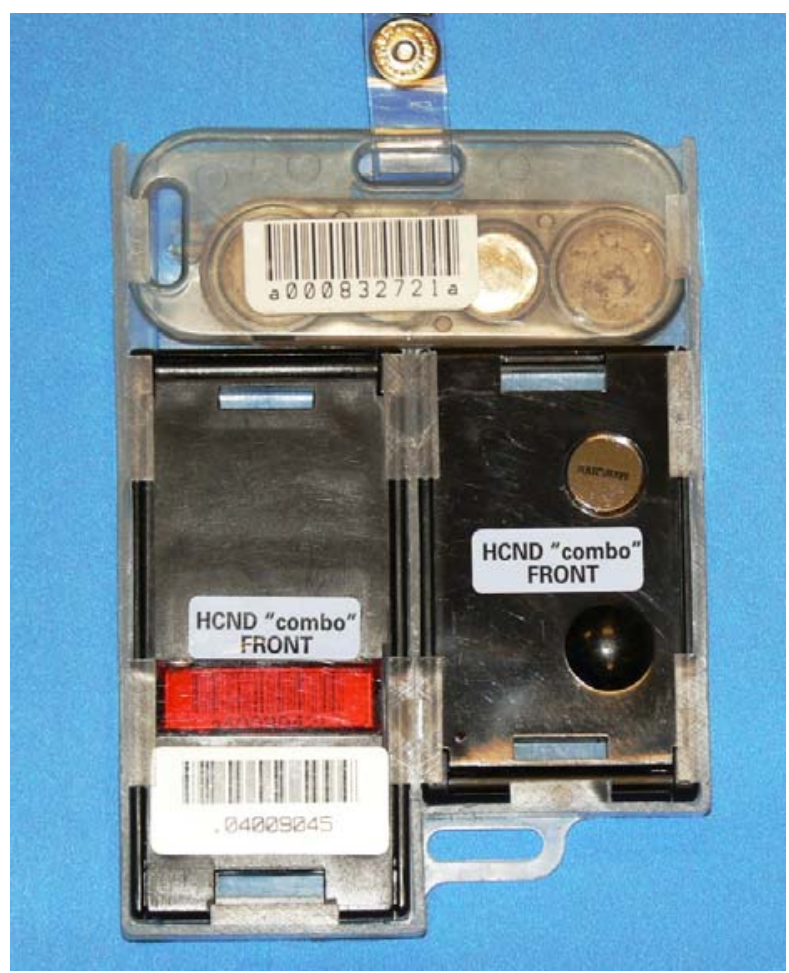

Figure 6.9 HCND with PNAD - Front Side

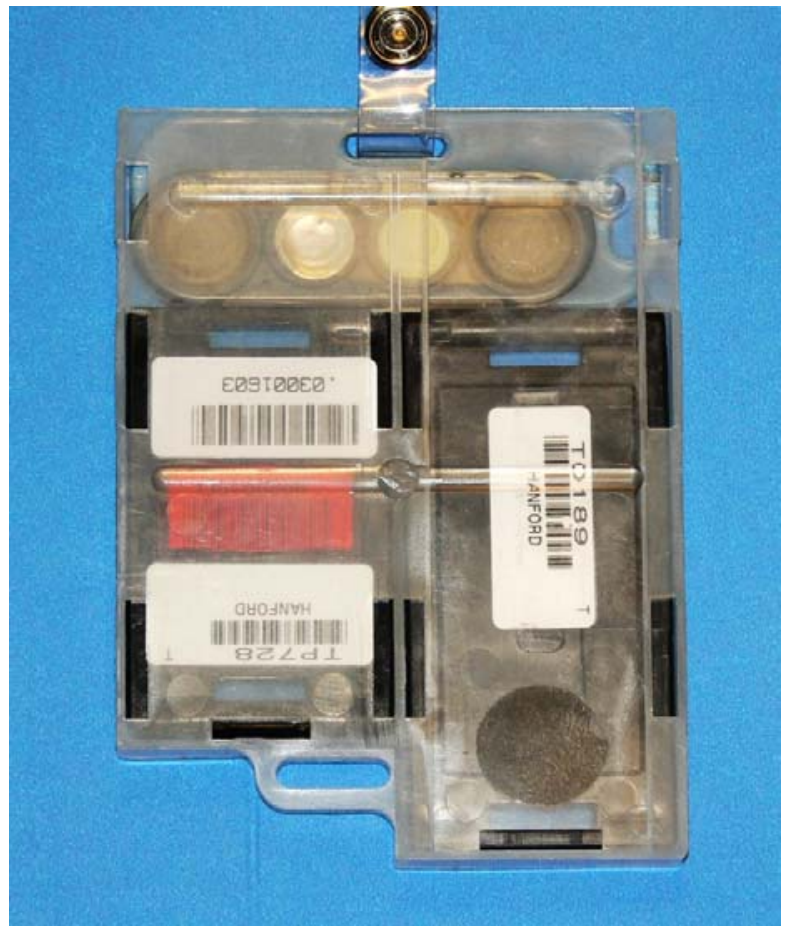

Figure 6.10 HCND with PNAD - Back Side 


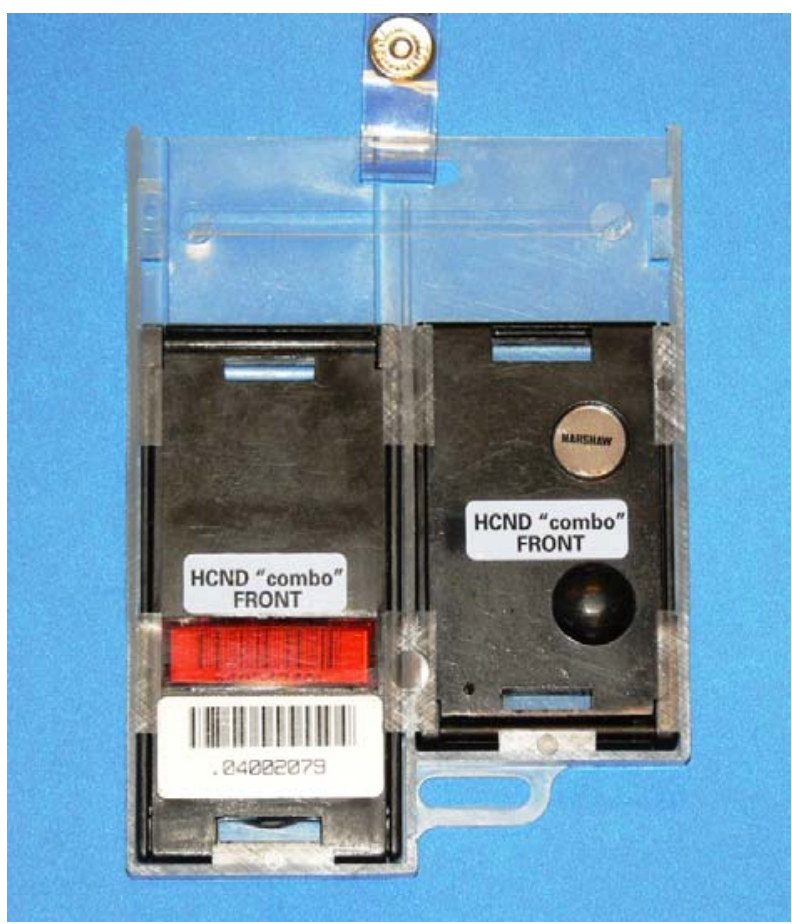

Figure 6.11 HCND without PNAD - Front Side

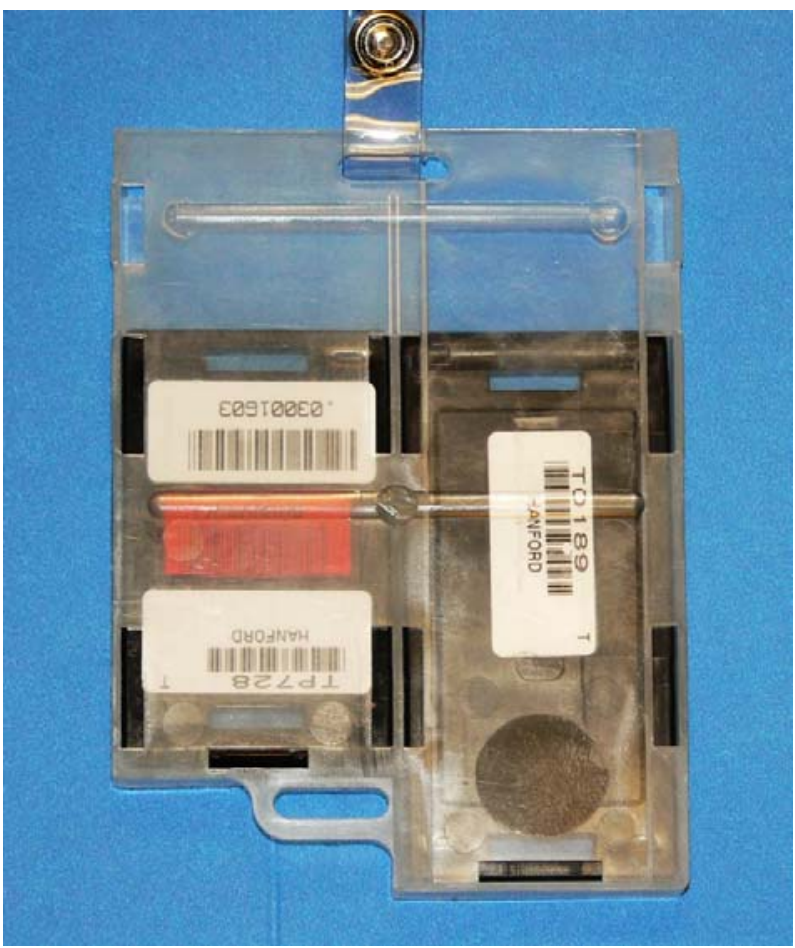

Figure 6.12 HCND without PNAD - Back Side 


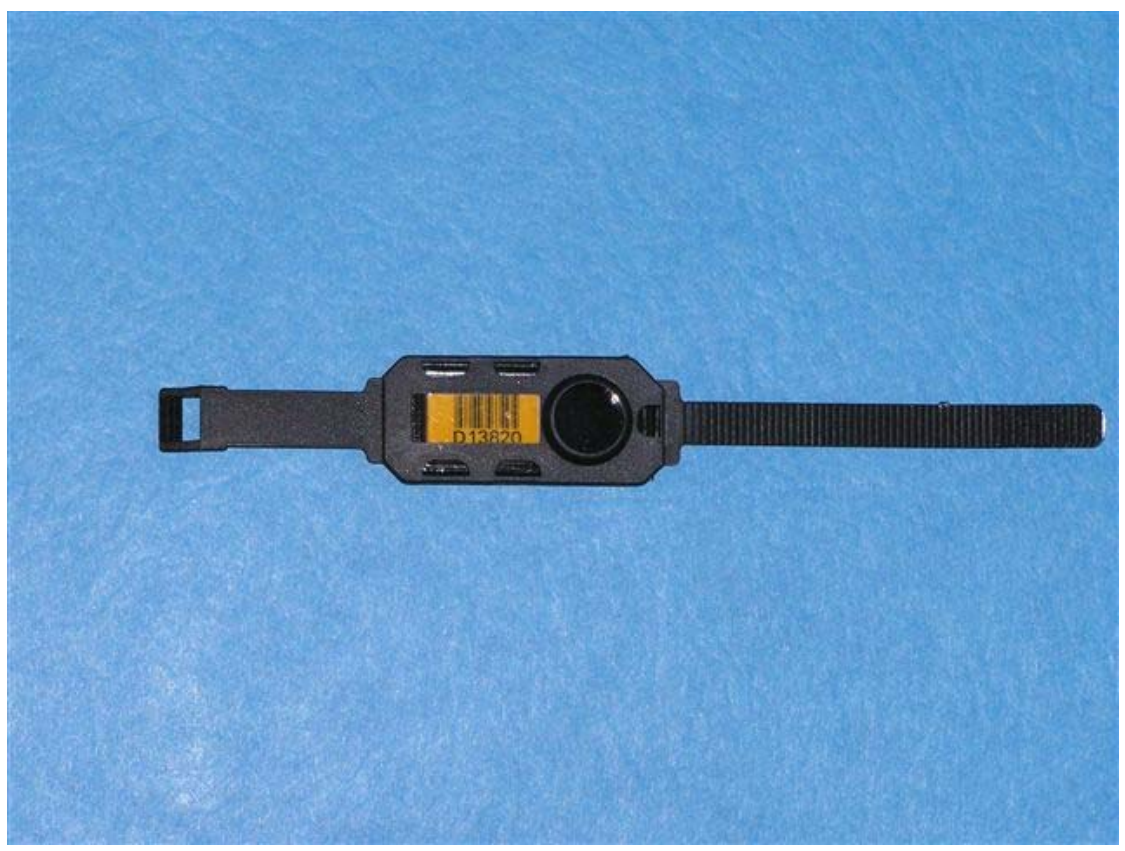

Figure 6.13 EXT-RAD Finger Ring- Front

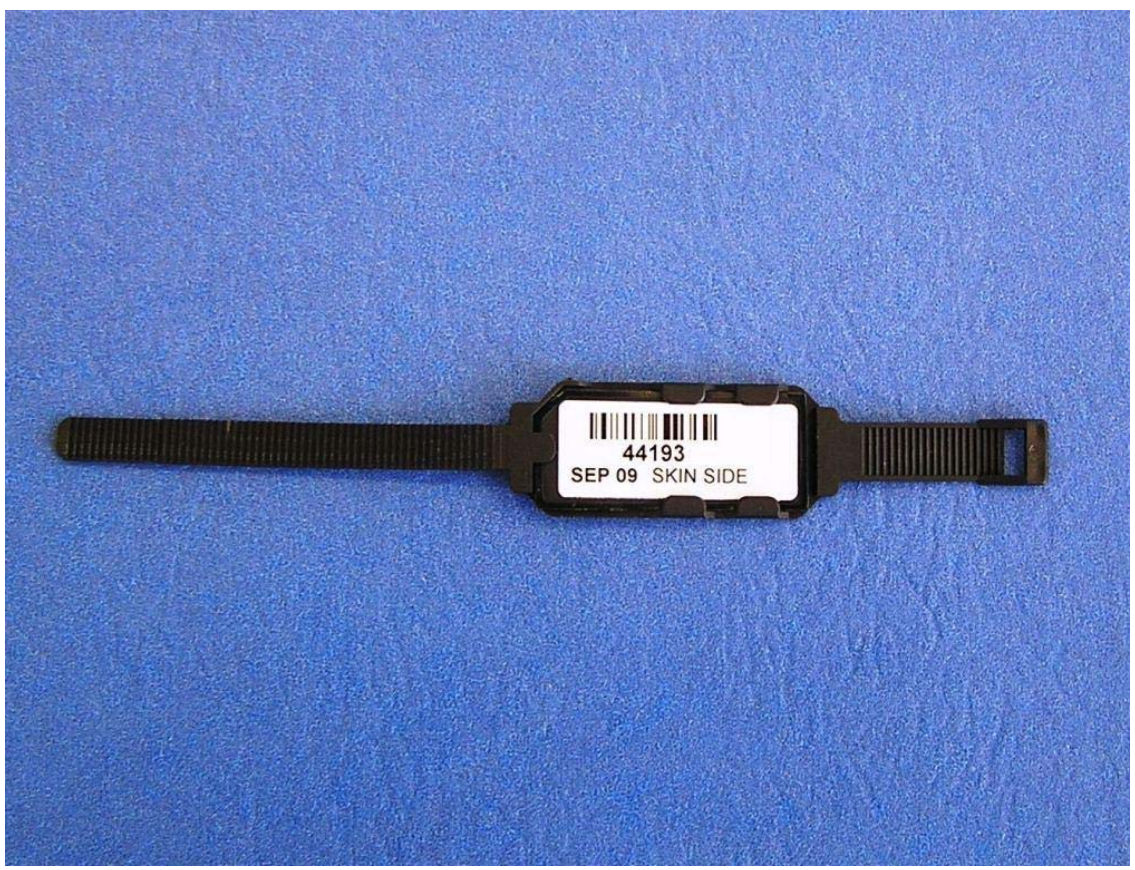

Figure 6.14 EXT-RAD Finger Ring-Back 


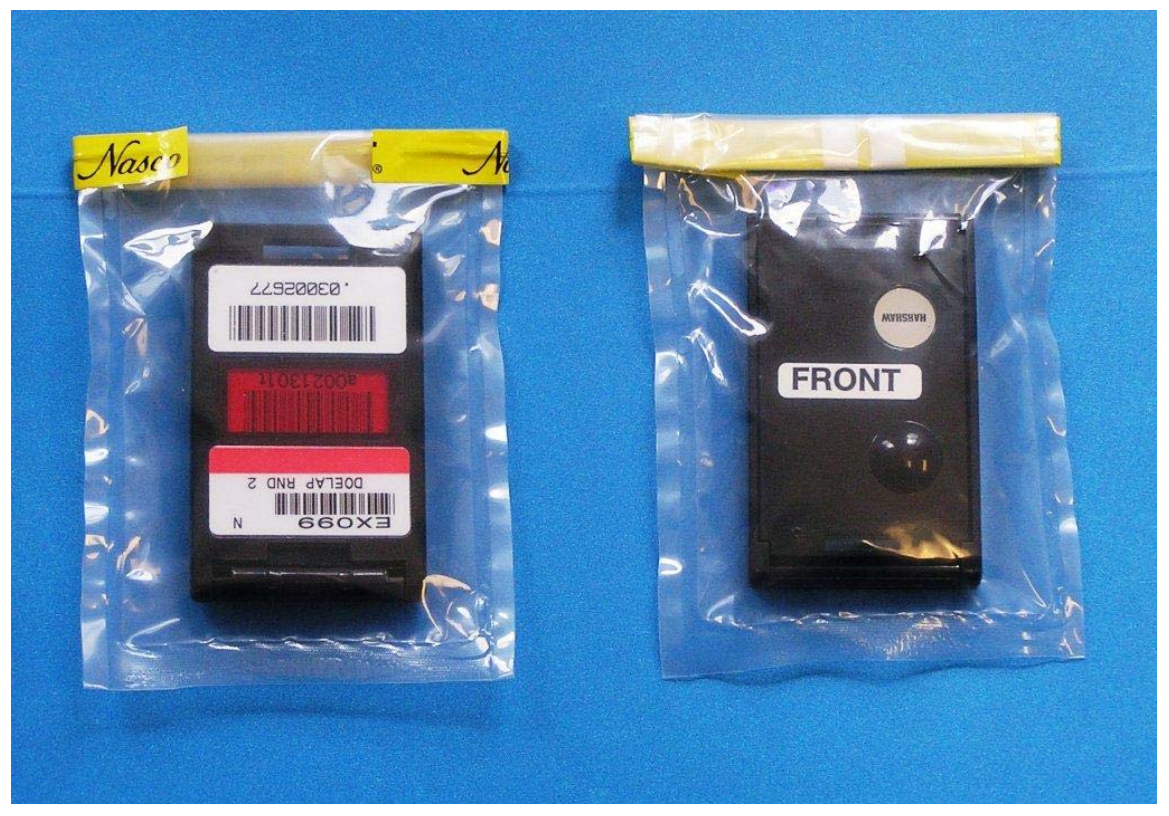

Figure 6.15 HSD in Whirl-Pak ${ }^{\circledR}$ plastic bag

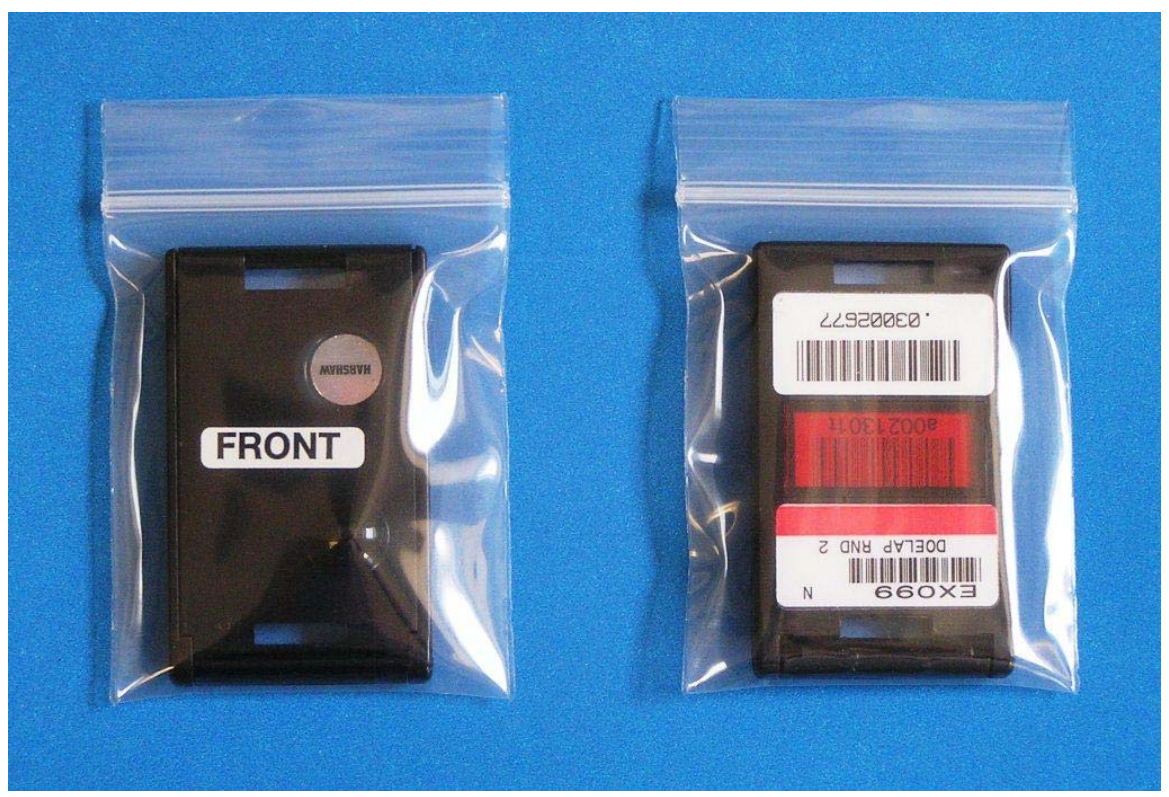

Figure 6.16 HSD in Ziploc ${ }^{\circledR}$ plastic bag ${ }^{(a)}$

(a) Ziploc ${ }^{\circledR}$ is a registered trademark of S.C. Johnson \& Son, Inc. All rights reserved. 


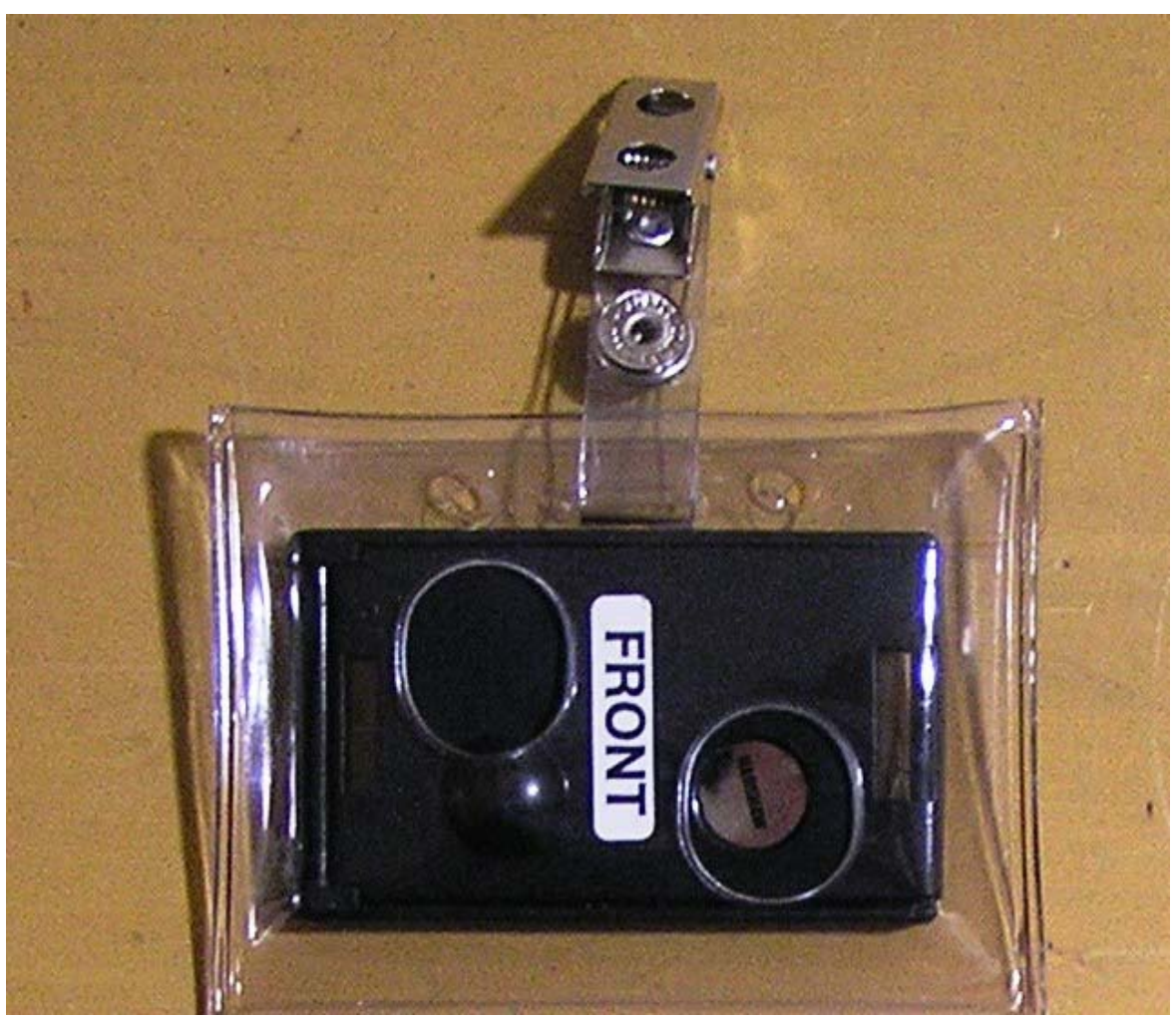

Figure 6.17 HSD in Vinyl Pouch - NOT APPROVED 


\subsection{Field Measurements, Assessments, and Intercomparison Studies}

Because of shortfalls in dosimetry technology, comparisons of dosimeter measured dose with dose measured by independent means such as electronic dosimeters, survey instruments, or specialized field instrumentation is generally advised to verify the accuracy of the dosimeter reported dose in Hanford work environments. Field measurements that include fluence and energy spectra are generally the most valuable for this purpose, but also the most difficult to perform. For photon radiation fields, Hanford personnel dosimeters provide reasonably accurate results over a wide range of energies. Confirmatory field measurements are generally not necessary except for the relatively rare situations involving photon energies less than $16 \mathrm{keV}$ or greater than $3 \mathrm{MeV}$. For beta radiation fields, Hanford personnel dosimeters provide relatively accurate response if the average beta energy is greater than $250 \mathrm{keV}$. For radiation fields with beta energies less than $250 \mathrm{keV}$, confirmatory field measurements may be required to verify proper performance and/or correction factors to be applied. For neutron radiation fields, albedo neutron TLDs have an extremely energy dependent response. For Hanford dosimeters with a single neutron sensitive TLD element such as the HSD, neutron response can vary over two orders of magnitude. For multi-element dosimeters such as the 8816 dosimeter, energy dependence is less severe with energy compensating algorithms but field measurements may still be necessary to verify dosimeter accuracy. Technical reports of field measurements (Fix et al. 1981, 1982; Brackenbush et al. 1980, 1991; Endres et al 1996; Scherpelz, Fix, and Rathbone 2000), along with numerous letter reports to HEDP files, have been prepared.

\subsection{Methodology}

Since the adoption by DOE (DOE 2007a) of a system of protection quantities (effective dose and equivalent dose) that differ in fundamental ways from the operational quantities (personal and ambient dose equivalent) traditionally used to monitor personnel and workplace environs, there have been two objectives to field measurements. One is to verify that the dosimeter accurately measures the operational quantities it is designed and calibrated to measure. The other is to verify that the operational quantities being measured are conservative estimators of the protection quantities used by regulators in their system of dose limitation. This latter objective applies primarily to neutron radiation fields.

For the purpose of assessing dosimeter accuracy in measuring personal dose equivalent in workplace radiation fields, field measurements usually involve exposure of personnel dosimeters on phantom and exposure of specialized instruments separately at the same location in the radiation field, and ultimately a comparison of values measured by the reference instrument and the values reported by the dosimeter. Sometimes the instrument is designed to have a relatively energy independent personal dose equivalent response, so that it can be used to measure the operational quantity directly. More often, the quantities actually "measured" by the reference instrument are physical quantities such as fluence and fluence energy spectrum which can be used to calculate the operational quantities that are measured and reported by the dosimeter. 
For the purpose of assessing whether the operational quantities are conservative relative to the protection quantities for the particular radiation fields in which workers are being monitored, only reference instruments are required for field measurements. Dosimeters are not required. Measurements of fluence and fluence energy spectrum can be used to calculate both operational quantities and protection quantities using conversion coefficients from ICRU 57. For the calculations of both the operational quantities and the protection quantities, the angular distribution of the fluence needs to be either known or assumed.

For the purpose of verifying dosimeter performance in the workplace, measurements of the fundamental quantities fluence and fluence energy spectrum have advantages over direct measurements of personal dose equivalent. Fluence to dose equivalent conversion factors and fluence to effective dose conversion factors have been internationally accepted and published in references such as ICRU 57. Fluence data are portable. When quality factors and the Q-LET relationship defining them change or when new operational quantities or protection quantities are defined, the fundamental fluence data obtained during past field measurements can be used to recalculate the new quantities once the appropriate conversion factors have been made available. The need to repeat field measurements when field conditions are unchanged, simply to reassess compliance with a new system of operational or protection quantities, is largely eliminated.

\subsubsection{Photon Radiation}

For measurements in photon fields, the reference instruments are typically ionization chambers and solid state gamma spectroscopy systems. For measurements in photon radiation fields, the physical quantities "measured" by the reference instruments are typically air kerma, photon fluence and fluence energy spectrum. These physical quantities can be used to calculate the operational quantities reported by personnel and environmental dosimeters. For proper calculation of personal dose equivalent $\mathrm{H}_{\mathrm{p}}(\mathrm{d})$, the angular distribution of the fluence must either be known or assumed. For calculation of ambient dose equivalent $\mathrm{H}^{*}(10)$, the angular distribution does not need to be known.

\subsubsection{Beta Radiation}

For measurements in beta radiation fields, the reference instruments typically used are extrapolation chambers or solid state beta spectroscopy systems. The physical quantities "measured" by the reference instruments are typically absorbed dose in air (converted to absorbed dose in tissue) at a fixed depth, or beta particle fluence and fluence energy spectrum, which can be used to calculate the absorbed dose and dose equivalent in tissue at a specified depth. For contact measurements of point sources, other methods of dose assessment may be used, such as of photographic film (i.e., autoradiography), thin thermoluminescent phosphors, etc. 


\subsubsection{Neutron Radiation}

For measurements in neutron radiation fields, the reference instruments typically used are the tissue equivalent proportional counter (TEPC) and the multisphere spectrometer system. A device similar to the multisphere system called ROSPEC is sometimes used. The ROSPEC uses proton recoil detectors.

For proper calculation of personal dose equivalent $\mathrm{H}_{\mathrm{p}}(\mathrm{d})$, the angular distribution of the fluence must either be known or assumed. For calculation of ambient dose equivalent $\mathrm{H}^{*}(10)$, the angular distribution does not need to be known.

When making neutron measurements in rooms with a large scatter component to the fluence, caution must be exercised when comparing the personal dose equivalent measured by the dosimeter and by the instrument. Instrument measurements are usually made with the detector free in air, whereas dosimeter measurements must be made on phantom. The instrument response is isotropic and the detector "sees" fluence from all directions, whereas the dosimeter only "sees" fluence" coming from the direction in which the dosimeter and phantom are "facing" because of the $2 \pi$ shielding effect of the phantom. The dosimeter is calibrated to accurately report dose in a parallel beam or AP geometry where fluence is normally incident on the front face of the phantom. Therefore, the fluence to dose equivalent conversion factors used with the multisphere unfolded spectrum generally assume parallel beam or AP geometry. Because the multisphere detector "sees" a larger fluence than the dosimeter, the $H_{p}(10)_{\eta}$ calculated with the multisphere will be larger than the $H_{p}(10)_{\eta}$ reported by the dosimeter. In neutron radiation fields where there is a large amount of scatter off walls, floor or ceiling, or there are multiple sources in different directions, the quantities that can be meaningfully assessed with instrumentation are ambient dose equivalent, $H^{*}(10)$, effective dose for an isotropic exposure geometry $\mathrm{E}_{\text {Iso, }}$, and effective dose for a rotational exposure geometry $\mathrm{E}_{\mathrm{ROT}}$. Field measurements of $\mathrm{H}_{\mathrm{p}}(10)_{\eta}$ with isotropic responding instruments are best made in low scatter environments in AP geometry with single, well defined point sources.

\subsubsection{Multisphere Spectrometer}

The multisphere spectrometer system uses a $1.3-\mathrm{cm}-(0.5-\mathrm{in}$.$) diameter by 1.3-\mathrm{cm}$ (0.5-in.) ${ }^{6} \mathrm{LiI}(\mathrm{Eu})$ scintillation detector in conjunction with different sized polyethylene moderator spheres. The spectrum unfolding code MAXED (Reginatto and Goldhagen, 1998) is used in conjunction with neutron response functions for the ${ }^{6} \mathrm{LiI}(\mathrm{Eu})$ detector with each sized sphere, to unfold the neutron energy spectrum. The fluence energy spectrum thus obtained is used to calculate the desired operational quantity.

Prior to conducting field measurements, calibration laboratory measurements are generally conducted with these detectors exposed to well characterized neutron fields to verify the calibration of the system. The accuracy of the spectrum unfolding technique is validated by comparison of the unfolded spectrum with the "known" spectrum.

When $H_{p}(10)_{\eta}, H^{*}(10)_{\eta}$ or $E_{\eta}$ are calculated from neutron fluence and energy spectrum measurements, the calculated dose includes the gamma dose from 
gammas originating within the body or phantom from capture reactions. The contribution to total dose from neutrons can be significant when there is a large thermal component to the neutron energy spectrum.

A disadvantage to measurements with multisphere spectrometer systems is the fact that the shape of the energy spectrum that is unfolded is dependent on starting assumptions about what the spectrum should look like. Considerable uncertainty in calculated dose can be introduced as a result of errors in the unfolded spectrum. For this reason, dose measurements from unfolded spectra are generally confirmed by modeling, or independent measurements using other means (e.g. TEPC).

\subsubsection{Tissue Equivalent Proportional Counter (TEPC)}

The TEPC is an ionization chamber with tissue equivalent walls and tissue equivalent fill gas, operated in the proportional region. The fill gas is at very low pressure such that the mass represented by the sensitive volume of the detector is approximately equal to a $2 \mu$ diameter sphere of tissue. Because the conditions for cavity theory are satisfied, the charge collected in the gas is proportional to the absorbed dose from neutrons in the detector walls. The counter also records a pulse height spectrum where pulse height is proportional to the lineal energy of charged particle ionization tracks in the chamber. The lineal energy spectrum is converted to a Linear Energy Transfer (LET) spectrum through well established mathematical relationships (Scherpelz and Conrady, 2008). The LET spectrum allows calculation of an average quality factor to be applied to the absorbed dose.

The operational quantity that is measured with the TEPC is less well defined than with the multisphere. The TEPC detector only "sees" events generated within it's walls. In parallel beam exposure geometry, the detector is not exposed to backscattered neutrons that would otherwise be generated by the presence of a body or phantom. The dose measured does not correspond to the dose at any particular depth in a phantom or body. If a depth were to be assigned, it would be the depth of the chamber walls, on the order of a few hundred $\mathrm{mg} / \mathrm{cm}^{2}$ in density thickness. Of the operational quantities defined by ICRU, the TEPC quantity most closely resembles ambient dose equivalent from neutrons.

Although the TEPC has distinct advantages in being able to simultaneously measure dose from both photons and neutrons, isolating the contribution from each using the LET spectrum, it has generally not been configured in this manner for measurements at Hanford. As configured for most measurements at Hanford, the TEPC only measures the charged particle dose directly arising from neutron interactions in the body. It does not account for gamma dose from capture reactions within the body, which can be significant with highly thermalized spectra. 


\subsection{Hanford Beta Radiation Measurements}

Numerous technical studies and measurements have been conducted of beta radiation in Hanford facilities. Technical reports of primary interest include the following:

- J. J. Fix et al. 1981. Hanford Personnel Dosimeter Supporting Studies FY-1980. PNL-3536. Pacific Northwest Laboratory.

- J. J. Fix et al. 1982. Hanford Personnel Dosimeter Supporting Studies FY-1981. PNL-3736. Pacific Northwest Laboratory.

- HEDP File: C. D. Hooker et al. Skin Dose Assessment from Extrapolation Chamber Measurements of Contaminated Clothing. July 1985.

- HEDP File: L. A. Rathbun, K. L. Swinth, and D. L. Haggard. Beta Measurements at Hanford. April 1986.

- HEDP File: L. A. Rathbun. Beta Measurements at PUREX. September 16, 1988.

- HEDP File: J. J. Fix, PNL, to D. P Higby, PNL. Extremity Dose Evaluation of Waste Tank Sample Handling in the 325 Building. June 28, 1995

- HEDP File: J. J. Fix. Extremity Dose Evaluation of Yttrium-90 Purification Process in 325 Building. September 12, 1990.

- HEDP File: B. A. Rathbone, J. J. Fix, A. W. Endres, PNL, and D. S. Cunningham, WHC. Evaluation of Extremity Dose Associated with Handling Waste Tank Sludge Samples at the Westinghouse Hanford Company 222-S Facility. January 22, 1996.

- HEDP File: J. J. Fix, PNL, to W. A. Decker, Jr., WHC. Extremity Dosimeter Facility Calibration Factors. January 26, 1996.

- HEDP File: B. A. Rathbone, PNL, to L. K. Aldrich, WHC. Special Evaluation of Ring Results in 241-AZ-101 Thermocouple Incident. February 26, 1996.

- HEDP File: B. A. Rathbone, PNL, to L. R. McKay, WHC. Special Evaluation of HSD Results for 241-AZ-101 Thermocouple Incident. March 4, 1996.

- HEDP File: B. A. Rathbone, PNL, to L. R. McKay, WHC. Assessment of Ring Correction Factors for 241-AZ-101 Incident. March 4, 1996.

- HEDP File: B. A. Rathbone, PNL, to J. M. Hammack, Lockheed Martin Hanford Corporation. Determination of Ring Correction Factors and 
Dose Reduction Factors for Leaded Gloves Used in Grab Sampling Activities at Hanford Tank Farms, July 10, 1997.

- HEDP File: B. A. Rathbone, PNL, to HEDP file, Assessment of Ring Correction Factors for Use at Hanford. November 30, 1998. Also published in Proceedings of Bicron/NE TLD Users Symposium, March 1998.

- HEDP File: B. A. Rathbone, PNL, to Nancy Kirner, FDH. Measurement of Shallow Dose Rate in the Beam of T-Handle Sample Carriers. March 7, 2000.

- B. A. Rathbone et al. 2002. Current Challenges in Personnel Dosimetry at the U.S. DOE Hanford Site. Radiation Protection Dosimetry Vol. 101. Nos. 1-4, pp. 153-166 (2002)

These studies illustrate the strong dependence of personnel dose from beta radiation on energy and the geometry of irradiation. Conditions where beta radiation may be a concern generally involve inspection, repair, or maintenance of contaminated equipment. Often in these cases, mixed activation and/or fission products are present, resulting in both beta and photon radiation. Contractor personnel using portable survey instruments can easily identify these locations. Another type of facility where beta radiation can be a concern involves laboratories responsible for sample analysis. Significant extremity doses may occur if samples of pure beta-emitting nuclides are handled. At Hanford, significant quantities of the beta-only-emitting nuclides ${ }^{147} \mathrm{Pm},{ }^{90} \mathrm{Y}$, and ${ }^{90} \mathrm{Sr}$ have been handled.

\subsection{Hanford Photon Radiation Measurements}

Photon radiation typically is associated with beta and/or neutron radiation in Hanford facilities. The majority of personnel radiation exposure at Hanford is attributable to photon radiation because of its relative abundance and difficulty to shield. Studies of this radiation in Hanford facilities include the following:

- J. J. Fix et al. 1981. Hanford Personnel Dosimeter Supporting Studies FY-1980. PNL-3536. Pacific Northwest Laboratory.

- J. J. Fix et al. 1982. Hanford Personnel Dosimeter Supporting Studies FY-1981. PNL-3736. Pacific Northwest Laboratory.

- HEDP File: P. L. Roberson and F. M. Cummings. Gamma Measurements at the 234-5 Facility. October 1986.

- HEDP File: L. L. Nichols, PNL, to Bill Decker, WHC. Photon Measurements at PUREX. August 1, 1988.

- HEDP File: B. A. Rathbone, PNL, to W. A. Decker, Jr. WHC. Evaluation of 106C Dosimeter Results for Evidence of Low Energy Photon Exposure. October 6, 1995. 
- HEDP File: B. A. Rathbone, PNL, to W. A. Decker, Jr., WHC. Analysis of 106C Test Dosimeter for Low Energy Photon Exposure. November 11, 1995.

- HEDP File: B. A. Rathbone, PNL, to HEDP file. Estimating Average Photon Energy From HSD Element Ratios, February 4, 1997.

Based on the dose response characteristics of Hanford dosimeters presented in Chapter 5, there is no reason to expect difficulty in measuring personnel dose from photon radiation. The foregoing studies provide information on the intensity and energy distribution of photons observed in Hanford work environments. The majority of these studies were focused on isolating the dose attributable to beta and/or neutron radiation, where problems in measuring dose may occur, from the dose attributable to photon radiation, which is expected to be relatively free of error. These measurements confirm the substantial confidence in dosimeter results for this form of radiation.

\subsection{Hanford Neutron Radiation Measurements}

The vast majority of Hanford instrument measurements of dose in the work environment have been conducted for neutron radiation since this is where the greatest technology shortfall exists in personnel dosimetry. Selected studies of neutron dose measurements include the following:

- J. J. Fix et al. 1981. Hanford Personnel Dosimeter Supporting Studies FY-1980. PNL-3536. Pacific Northwest Laboratory.

- J. J. Fix et al. 1982. Hanford Personnel Dosimeter Supporting Studies FY-1981. PNL-3736. Pacific Northwest Laboratory.

- HEDP File: P. L. Roberson, F. M. Cummings, and J. J. Fix. Neutron and Gamma Field Measurements at the 234-5 Facility." September 1985.

- HEDP File: P. L. Roberson, F. N. Eichner, and K. L. Jones. Evaluation of a Wrist Dosimeter Based on Hankins' Design. May 1986.

- HEDP File: F. M. Cummings, and L. L. Nichols. Neutron Field Measurements at the 234-5 Facility. October 1986.

- HEDP File: L. W. Brackenbush et al. Neutron Dose and Spectrum Measurements in Westinghouse Hanford Facilities. September 1987.

- J. J. Fix, W. V. Baumgartner, L. W. Brackenbush, L. L. Nichols, T. J. Paul, and A. W. Endres. 1991. Hanford Personnel Neutron Dosimetry Problems and Solutions. CONF-9106235/ PNL-SA-21596, Eleventh DOE Workshop on Personnel Neutron Dosimetry, pp. 33-42, June 3-7, 1991. 
- $\quad$ L. W. Brackenbush, W. V. Baumgartner, and J. J. Fix. 1991. Response of TLD-Albedo and Nuclear Track Dosimeters Exposed to Plutonium Sources. PNL-7881. Pacific Northwest Laboratory.

- $\quad$ A. W. Endres, L. W. Brackenbush, W. V. Baumgartner, and B. A. Rathbone. 1994. Site Specific Calibration of the Hanford Personnel Neutron Dosimeter. ORNL/TM-12817, Proceedings of Fourth Conference on Radiation Protection and Dosimetry, pp. 153-161, October 23-27, 1994, Orlando, Florida.

- A. W. Endres, L. W. Brackenbush, W. V. Baumgartner, J. J. Fix, and B. A. Rathbone. 1996. Response of the Hanford Combination Neutron Dosimeter in Plutonium Environments. PNL-10516. Pacific Northwest Laboratory.

- HEDP File: W. A. Baumgartner, PNL, to DS Cunningham, WHC. A Study of Tank Farm Workers using High Energy Neutron Source. January 21, 1994.

- HEDP File: W. A. Baumgartner. Facility Factor for Westinghouse Hanford Company PFP Facility. January 9, 1995.

- HEDP File: B. A. Rathbone. Technical Equivalence of TLD and TED in the Hanford Combination Neutron Dosimeter. February 22, 1995.

- $\quad$ R. I. Scherpelz, J. J. Fix, and B. A. Rathbone. January 31, 2000. Validation of Hanford Personnel and Extremity Dosimeters in Plutonium Environments. PNNL-13136 Pacific Northwest National Laboratory.

- HEDP File: B. A. Rathbone. A New 8816 Algorithm for PFP. December 18, 2000.

- HEDP File: B. A. Rathbone. Recommendations on the Use of HCNDs with Lead Aprons at PFP. September 30, 2003

- HEDP File: R. I. Scherpelz and B. A. Rathbone. Neutron Measurements at PFP August - September, 2003. November 14, 2003.

- HEDP File: R. I. Scherpelz. Neutron Measurements on the ISA Pad. December 9, 2003

- HEDP File: B. A. Rathbone. Correction Factors for Area HSD and Area HCND Neutron Dose Results at SNF Facilities. December 31, 2003.

- HEDP File: R. J. McConn and R. I. Scherpelz. MCNP Estimate of Dose Rates Surrounding the ISA Pad. January 23, 2004.

- HEDP File: B. A. Rathbone. Neutron Correction Factors for HSD Area Dosimeters Located in the Vicinity of the ISA in 200 East Area.

February 12, 2004. 
- HEDP File: B. A. Rathbone. Neutron Response of HSD and HCND Personnel Dosimeters Near Spent Fuel Casks. February 13, 2004.

- HEDP File: B. A. Rathbone. Verification of 8816 Performance in PFP Neutron Fields. March 3, 2004.

- $\quad$ Scherpelz R. I., and M. M. Conrady. 2008. Upgrade of the PNNL TEPC and Multisphere Spectrometer. PNNL-17809, Pacific Northwest National Laboratory, Richland, WA.

- $\quad$ Conrady MM, RK Berg, RI Scherpelz, and BA Rathbone. 2009. Neutron Measurements at Hanford's Plutonium Finishing Plant. PNNL18649, Pacific Northwest National Laboratory, Richland, WA.

Information in the foregoing studies demonstrates the complexity of measuring neutron dose and comparing the instrument-measured dose to the dosimeterinterpreted dose. Throughout the history of the Hanford TLD program, facility or site specific calibration factors have been used because of the significant energy response of TLD albedo dosimeters. Because of the limited capabilities of albedo neutron dosimeters, instrument measurements in the work environment are crucial to ensuring the adequacy of the dosimeter-interpreted neutron dose.

\subsection{Hanford Environmental Radiation Measurements}

Environmental TLDs are routinely used to measure the environmental dose at several selected onsite and offsite locations. Confirmatory measurements of these data are available in the following:

- $\quad$ L. A. Rathbun. 1989. The Determination of the Penetrating Radiation Dose at Hanford. PNL-7124. Pacific Northwest Laboratory.

- HEDP File: A. W. Endres. 1994. Results of the 1991 Environmental Radiation Quality Assurance Task Force of the Pacific Northwest Thermoluminescent Dosimeter Intercomparison. May 26, 1994.

- B. A. Rathbone, A. W. Endres, and E. J. Antonio. 1994. Evaluation of New and Conventional Thermoluminescent Phosphors for Use in Routine Environmental Monitoring Programs Using Automated Readers. ORNL/TM-12817, Proceedings of Fourth Conference on Radiation Protection and Dosimetry, pp. 371-380, October 23-27, 1994, Orlando, Florida. 


\subsection{Intercomparison Studies}

Hanford routinely participates in external dosimeter performance intercomparison studies. These studies are critically important to ensuring the adequate performance of the system. Hanford has participated in the following intercomparison studies in recent years:

- HEDP File: Summary of PNL's Participation in the DOE Draft Performance Standard for Extremity Dosimeters. February 20, 1990.

- HEDP File: Summary of PNL Performance in DOELAP Dosimeter Performance Testing During 1991. March 24, 1992.

- HEDP File: Environmental Radiation Quality Assurance Task Force of the Pacific Northwest Thermoluminescent Dosimeter Intercomparison. September 15, 1992.

- HEDP File: Summary of PNL's Results from Participation of Hanford in the 18th Personnel Dosimeter Intercomparison Study. July 1993.

- HEDP File: Summary of PNL Performance in DOELAP Dosimeter Performance Testing during 1994. August 15, 1994.

- HEDP File: PNL's Participation in the Tenth International Intercomparison Project. August 24, 1994.

- HEDP File: Summary of PNL Performance in DOELAP Dosimeter Performance Testing during 1996. June 19, 1996.

- HEDP File: DOELAP ${ }^{204} \mathrm{Tl} /{ }^{137} \mathrm{Cs}$ Dosimeter Intercomparison - (OARMRESL-98-234).

- $\quad$ HEDP File: $11^{\text {th }}$ International Environmental Dosimetry Intercomparison.

- HEDP File: $12^{\text {th }}$ International Environmental Dosimetry Intercomparison.

- HEDP File: ANSI/HPS N13.29 Pilot Test

- HEDP File: ANSI/HPS N13.112001 Pilot Test.

These intercomparison studies all show acceptable or exemplary performance of Hanford dosimetry systems. ANSI/HPS N13.29 (HPS 1996b) is a draft performance test standard for Environmental Dosimetry which has not yet been issued as of this writing. 


\subsection{Uncertainty Analyses}

Studies have been performed to estimate the bias and uncertainty in Hanford recorded dose. These evaluations have shown improved performance of Hanford dosimetry systems from the 1940s through the present time. Notable studies include the following:

- J. J. Fix, and E. S. Gilbert. 1991. Consistency of External Dosimetry in Epidemiologic Studies of Nuclear Workers. ORNL/TM-11881.

Proceedings of the Third Conference on Radiation Protection and Dosimetry. October 1991.

- J. J. Fix, E. S. Gilbert, R. H. Wilson, W. V. Baumgartner, and L. L. Nichols. 1992. Comments on Evidence of Biased Recording of Radiation Doses of Hanford Workers. Letter to the Editor, American Journal of Industrial Medicine, volume 22, pp. 281-283.

- J. J. Fix, and E. S. Gilbert. 1992. Consistency of External Dosimetry in Epidemiologic Studies of Nuclear Workers. IRPA8, Proceedings of the 8th Meeting of the International Radiation Protection Association, volume 1, pp. 567-570, May 17-22, 1992.

- J. J. Fix, E. S. Gilbert, and W. V. Baumgartner. 1994. Estimates of Bias and Uncertainty in Recorded Dose. ORNL/TM-12817, Proceedings of Fourth Conference on Radiation Protection and Dosimetry, pp. 119-125, October 23-27, 1994, Orlando, Florida.

- J. J. Fix, E. S. Gilbert, and W. V. Baumgartner. 1994. An Assessment of Bias and Uncertainty in Recorded Dose from External Sources of Radiation for Workers at the Hanford Site. PNL-10066. Pacific Northwest Laboratory.

- $\quad$ E. S. Gilbert and J. J. Fix. 1995. Accounting for Bias in Dose Estimates in Analyses of Data From Nuclear Worker Mortality Studies. Health Phys. 68(5):650-660.

- Fix, J. J., R. H. Wilson and W. V. Baumgartner. 1996. Retrospective Assessment of Personnel Neutron Dosimetry for Workers at the Hanford Site. PNNL-11196, Pacific Northwest National Laboratory, Richland, WA.

- $\quad$ Gilbert, E. S., and J. J. Fix. 1996. Laboratory Measurement Error in External Dose Estimates and Its Effects on Dose-Response Analyses of Hanford Worker Mortality Data. PNNL-11289, Pacific Northwest National Laboratory, Richland, WA.

- HEDP File: B. A. Rathbone Analysis of Uncertainty in 8825 Dosimeter Results, November 17, 1999.

- HEDP File: B. A. Rathbone 95\% Confidence Intervals for 8825 Dosimeter Results. November 22, 1999. 
- HEDP File: S. E. Huneycutt, Re-Evaluation of Measurement Uncertainty in the XD-740 Ring Dosimeter Results, March 30, 2000.

- HEDP File: S. E. Huneycutt, Uncertainty Documentation for the 8816 TLD and CR-39 Track Etch Dosimeters, October 8, 1999.

\subsection{Summary}

Uncertainty in recorded personnel dose is primarily a function of the radiation type, energy, dosimeter design, and irradiation geometry. Extensive efforts have been made at Hanford to document the performance of personnel dosimeters in actual work environments. These assessments have been made to estimate performance of the respective dosimeter systems under actual operational conditions. The following points summarize the current state of dosimetry technology:

- For photon radiation, which contributes the vast majority of personnel dose in Hanford facilities, the dosimeter-interpreted shallow, eye, and deep dose is considered accurate for uniform irradiation exposure geometries.

- For beta radiation greater than about $250 \mathrm{keV}$ (avg.), the dosimeterinterpreted shallow dose is considered accurate for uniform irradiation conditions. For non-uniform irradiation, discussed in Chapter 8.0, or for low-energy beta radiation, confirmatory field instrument measurements may be necessary.

- For neutron radiation, the dosimeter-interpreted whole body dose is considered accurate for uniform irradiation conditions where the neutron energy spectra are similar to the calibration spectra. For neutron spectra of either higher or lower energy, compared to the calibration spectra, confirmatory field and/or laboratory measurements may be necessary.

- For environmental radiation, the dosimeter-interpreted dose is considered accurate for uniform irradiation conditions, particularly for the higherenergy photon radiation (i.e., $>100 \mathrm{keV}$ ) typical of the energy spectra for naturally occurring environmental radiation. For beta radiation, special calibration of the dose algorithm and confirmatory field and or laboratory measurements are necessary.

These conclusions are consistent with the Hanford practice of conducting detailed instrument measurements of dosimeter performance in the work environment to document the accuracy of the recorded dose. 


\subsection{Assessment of Non-uniform Dose from Contamination on Skin or Clothing}

Non-uniform dose to the whole body, skin or extremities may result from discrete radioactive particles or distributed contamination on skin or clothing, point sources near the body, or collimated beams of radiation emerging from radiation generating devices, $\mathrm{x}$-ray diffraction units, electron microscopes, or charged particle accelerators. For dose from non-uniform external fields that can be monitored with multiple dosimeters, the methodology for assessing dose is described in Chapter 6. For dose from contamination on skin or clothing, the methodology for assessing dose is described in this chapter. In general, Hanford contractor radiation protection organizations are responsible for identifying cases of non-uniform exposure not adequately monitored by external dosimeters and for performing dose assessments in cases where the potential exists for localized shallow dose equivalent (SDE) or deep dose equivalent (DDE) to exceed 100 mrem. Assessment of equivalent dose to the skin and extremities from nonuniform exposures must be performed according to the methodology specified in 10 CFR 835.205.

Assessments of effective dose, E, should be performed in cases of non-uniform irradiation of portions of the whole body where the potential exists for the localized deep dose equivalent to exceed 100 mrem. For non-uniform exposures, the assessed localized deep dose equivalent will generally be conservative relative to effective dose and may be used for dose of record in lieu of effective dose calculations when practical considerations warrant. A possible exception to this rule may be skin contamination on or near the human testes that includes energetic beta emitters (see below).

Because whole body tissues of interest are primarily located at depths greater than $1 \mathrm{~cm}$, significant effective dose from external sources typically requires radiation with a substantial X-ray or gamma ray component. However, the human testes are a special case because the tissues at risk lie at depths between 0.2 and $0.8 \mathrm{~cm}$ (Casey et al 1982; Facey 1982, Charles 1986) and can potentially receive beta dose (e.g. from ${ }^{90} \mathrm{Y}$ ) as well as gamma dose from contamination on skin or clothing. Therefore, in rare cases of skin or clothing contamination involving the testes, it may be necessary to assess effective dose, E, that includes the contribution from external beta radiation in order to demonstrate compliance with 10 CFR 835.202(a)(1) and (2). In this case, a simple assessment of deep dose equivalent may not be adequate to demonstrate compliance with these two protection limits. 


\subsection{Action Levels for Skin Dose Assessment}

The DOE radiological control standard DOE-STD-1098-2008 Radiological Control (DOE 2008b) and Chapter 6 of the DOE Radiation Protection Programs Guide DOE G 441.1-1C Radiation Protection Programs Guide (DOE 2008a) recommend that action levels corresponding to 100 mrem be established for assessment of skin dose from skin or clothing contamination. Laboratory measurements were conducted to measure the response of the Eberline Model 260 pancake probe, Model RO-2 ion chamber, and Model RO-3B ion chamber to calibrated $0.2 \mathrm{~cm}^{2}$ disk sources and $225 \mathrm{~cm}^{2}$ slab sources. ${ }^{\text {(a) }}$ These instruments are commonly used at Hanford for radiological surveys. The disk sources were calibration sources routinely used to calibrate Hanford pancake probe instruments. The slab sources were used to simulate instrument response for cases where contamination is more extensive.

Instrument response data for these sources were used to establish the instrument readings corresponding to 100 mrem skin dose for contamination that has been resident on the skin for two hours. In accordance with 10 CFR 835.205(b) methods for dose assessment, doses were calculated to $1 \mathrm{~cm}^{2}$ and to $100 \mathrm{~cm}^{2}$ areas of skin depending upon the source size. Conservative action levels for each instrument corresponding to the DOE recommended action level of $100 \mathrm{mrem}$ are shown in Table 8.1. Tables 8.2, 8.3 and 8.6 summarize instrument response measurements and the original VARSKIN MOD2 calculations that were conducted to support the Table 8.1 values. ${ }^{\text {(a) }}$

Table 8.1. Portable Survey Instrument Action Levels for Skin Dose Assessment

\begin{tabular}{|c|c|c|}
\hline \multirow{2}{*}{ Instrument } & \multicolumn{2}{|c|}{ Readings for Potential Skin Dose $>100$ mrem $^{a}$} \\
\hline & $\begin{array}{c}\text { Contamination Area }< \\
\text { Probe Area }{ }^{\mathrm{b}}\end{array}$ & $\begin{array}{c}\text { Contamination Area }> \\
\text { Probe Area }{ }^{c}\end{array}$ \\
\hline Eberline Model 260 Pancake Probe & $2,500 \mathrm{cpm}$ & $40,000 \mathrm{cpm}$ \\
\hline Eberline Model RO-2 Ion Chamber (Open Window) ${ }^{\mathrm{d}}$ & $0.1 \mathrm{mrad} / \mathrm{h}$ & $5.5 \mathrm{mrad} / \mathrm{h}$ \\
\hline Eberline Model RO-3B Ion Chamber (Open Window) ${ }^{\mathrm{d}}$ & $0.06 \mathrm{mrad} / \mathrm{h}$ & $4.5 \mathrm{mrad} / \mathrm{h}$ \\
\hline \multicolumn{3}{|c|}{$\begin{array}{l}\text { a. Action levels are based on an assumed skin exposure time of } 2 \text { hours. } \\
\text { b. The action levels for Pancake GM are based on instrument response to } 0.2 \mathrm{~cm}^{2} \text { sources. The action levels for } \\
\text { ionization chambers are based on instrument response to } 0.2 \mathrm{~cm}^{2} \text { sources and } 2.0 \mathrm{~mm}^{2} \text { sources. The table } \\
\text { value is based on the most conservative case for Hanford radionuclides. } \\
\text { c. The action levels for all instrument types listed are based on instrument response to } 225 \mathrm{~cm}^{2} \text { sources. The } \\
\text { table value is based on the most conservative case for Hanford radionuclides. } \\
\text { d. These values are provided for information only; the pancake probe should be used unless readings exceed the } \\
\text { range of the pancake probe. }\end{array}$} \\
\hline
\end{tabular}

(a) Fix, J. J. "Minutes of Hanford Personnel Dosimetry Advisory Committee Meeting on December 14, 1994." (A copy is available in the Hanford Radiation Protection Historical Files, Pacific Northwest National Laboratory, Richland, Washington.) 
The following assumptions were used to determine these action levels:

- Contamination can be represented by one of two geometries:

Contaminated areas smaller than the probe - It is assumed that action levels based on instrument response to the $0.2 \mathrm{~cm}^{2}$ sources will be conservative for contamination areas smaller than the probe, including point sources as well as sources only slightly smaller than the probe. This assumption is supported by consideration of the effects of irradiation geometry on instrument response and on the actual shallow dose averaged over $1 \mathrm{~cm}^{2}$ of skin for different sized (area) contaminations under the probe.

Contaminated areas larger than the probe - It is assumed that the instrument reading is from a source considerably larger than the probe area and that the instrument responds primarily to activity under the probe window (i.e. probe walls attenuate most if not all of the beta radiation which contributes $>95 \%$ of the skin dose). As source area increases beyond the area of the probe window, the relationship between instrument reading and shallow dose rate under the probe (when averaged over areas of skin smaller than the area of the probe) remains relatively constant.

- Contamination is resident for a period of 2 hours.

- The probe survey is done at a distance of $1 \mathrm{~cm}$.

When an instrument measurement exceeds the respective action level in Table 8.1, or its equivalent expressed as cpm-hours (when exposure time is known), a skin dose assessment should be performed to ensure compliance with the requirements in 10 CFR 835.202(a)(4), 835.205(b) and 835.702(b). For the pancake probe, equivalent action levels for small and large sources which may be more useful are 5,000 cpm-h and $\mathbf{8 0 , 0 0 0} \mathbf{~ c p m}-\mathbf{h}$ respectively. Generally, it is recommended that data obtained from a pancake GM probe be used to determine the need for formal skin dose assessment. This is the standard instrument used for beta-gamma contamination measurement.

When one conservatively assumes a nominal efficiency of $10 \%$ for the pancake GM probe, as is typically done when documenting activity measurements on skin contamination forms, action levels of 50,000 dpm-h and 800,000 dpm-h can be derived for small and large sources respectively. When doing this however, it should be kept in mind that the efficiency for the pancake GM is actually between $20 \%$ and $30 \%$ for most radionuclides of interest at Hanford. As such, the indicated activities on skin contamination forms may overestimate the true activity by a factor of between 2 and 3. A VARSKIN dose calculation based on a 50,000 dpm-h skin exposure may result in a dose larger than 100 mrem, depending on the geometry and nuclide involved. Activity levels on the skin corresponding to 100 mrem, based on skin dose conversion factors published in the peer reviewed literature as well as VARSKIN 3 calculations, are given in Table 8.4 and Table 8.5. 
Table 8.2. Measured Instrument Response and Calculated Dose for Small $\left(0.2 \mathrm{~cm}^{2}\right)$ Radiation Sources

\begin{tabular}{|c|c|c|c|}
\hline Nuclide & $\begin{array}{c}\text { Eberline HP } 260 \text { Pancake } \\
\text { GM } \\
\text { Dose Rate/Reading } \\
((\mathrm{mrad} / \mathrm{h}) / \mathrm{cpm})^{\mathrm{a}, \mathrm{b}}\end{array}$ & $\begin{array}{c}\text { Eberline RO-2 Ionization } \\
\text { Chamber } \\
\text { Dose Rate/Reading } \\
\left([\mathrm{mrad} / \mathrm{h}]_{\mathrm{skin}} /[\mathrm{mrad} / \mathrm{h}]_{\mathrm{CP}}\right)^{\mathrm{a}, \mathrm{c}}\end{array}$ & $\begin{array}{c}\text { Eberline RO-3B Ionization } \\
\text { Chamber } \\
\text { Dose Rate/Reading } \\
\left([\mathrm{mrad} / \mathrm{h}]_{\text {skin }} /[\mathrm{mrad} / \mathrm{h}]_{\mathrm{CP}}\right)\end{array}$ \\
\hline${ }^{36} \mathrm{Cl}$ & $9.4 \times 10^{-3}$ & 130 & 173 \\
\hline${ }^{90} \mathrm{Sr} /{ }^{90} \mathrm{Y}$ & $2.0 \times 10^{-2}$ & 266 & 454 \\
\hline${ }^{99} \mathrm{Tc}$ & $1.1 \times 10^{-2}$ & 95 & 204 \\
\hline${ }^{137} \mathrm{Cs}$ & $1.6 \times 10^{-2}$ & 185 & 150 \\
\hline \multicolumn{4}{|c|}{$\begin{array}{l}\text { a. VARSKIN MOD2 calculated dose to } 1 \mathrm{~cm}^{2} \text {. } \\
\text { b. The most conservative case is for }{ }^{90} \mathrm{Sr} /{ }^{90} \mathrm{Y} \text { and results in a calculated action level of } 2500 \mathrm{cpm} . \text {.. } \\
\text { c. The most conservative case is for }{ }^{90} \mathrm{Sr} /{ }^{90} \mathrm{Y} \text { and results in a calculated action level of } 0.2 \mathrm{mrad} / \mathrm{h} \text {. } \\
\text { d. The most conservative case is for }{ }^{90} \mathrm{Sr} /{ }^{90} \mathrm{Y} \text { and results in a calculated action level of } 0.1 \mathrm{mrad} / \mathrm{h} \text {. }\end{array}$} \\
\hline
\end{tabular}

Table 8.3. Measured Instrument Response and Calculated Dose for Large $\left(225 \mathrm{~cm}^{2}\right)$ Radiation Sources

\begin{tabular}{|c|c|c|c|}
\hline Nuclide & $\begin{array}{c}\text { Eberline HP } 260 \text { Pancake } \\
\text { GM } \\
\text { Dose Rate/Reading } \\
((\mathrm{mrad} / \mathrm{h}) / \mathrm{cpm})^{\mathrm{a}, \mathrm{b}}\end{array}$ & $\begin{array}{c}\text { Eberline RO-2 Ionization } \\
\text { Chamber } \\
\text { Dose Rate/Reading } \\
\left([\mathrm{mrad} / \mathrm{h}]_{\mathrm{skin}} /[\mathrm{mrad} / \mathrm{h}]_{\mathrm{CP}}\right)^{\mathrm{a}, \mathrm{c}}\end{array}$ & $\begin{array}{c}\text { Eberline RO-3B Ionization } \\
\text { Chamber } \\
\text { Dose Rate/Reading } \\
\left([\mathrm{mrad} / \mathrm{h}]_{\mathrm{skin}} /[\mathrm{mrad} / \mathrm{h}]_{\mathrm{CP}}\right)^{\mathrm{a}, \mathrm{d}}\end{array}$ \\
\hline${ }^{90} \mathrm{Sr} /{ }^{90} \mathrm{Y}$ & $7.9 \times 10^{-4}$ & 8.4 & 10.6 \\
\hline${ }^{106} \mathrm{Ru} / \mathrm{Rh}$ & $1.2 \times 10^{-3}$ & 7.6 & 11.2 \\
\hline${ }^{137} \mathrm{Cs}$ & $4.5 \times 10^{-4}$ & 3.2 & 2.9 \\
\hline${ }^{204} \mathrm{Tl}$ & $9.5 \times 10^{-4}$ & 9.1 & 10.3 \\
\hline \multicolumn{4}{|c|}{$\begin{array}{l}\text { a. VARSKIN MOD2 calculated dose to } 100 \mathrm{~cm}^{2} \text {. } \\
\text { b. The most conservative case is for }{ }^{106} \mathrm{Ru} / \mathrm{Rh} \text { and results in a calculated action level of } 41,670 \mathrm{cpm} \text {.. } \\
\text { c. The most conservative case is for }{ }^{204} \mathrm{Tl} \text { and results in a calculated action level of } 5.5 \mathrm{mrad} / \mathrm{h} \text {. } \\
\text { d. The most conservative case is for }{ }^{106} \mathrm{Ru} / \mathrm{Rh} \text { and results in a calculated action level of } 4.5 \mathrm{mrad} / \mathrm{h}\end{array}$} \\
\hline
\end{tabular}

\subsection{Assessment of Dose to Skin or Extremities from Contamination}

Formal skin dose assessments should be performed using VARSKIN 3 (Durham 2006a) unless geometry or other considerations indicate the use of other methods. This code calculates dose equivalent, at a user specified depth in tissue, averaged over a user specified area of tissue. Doses from beta radiation can be calculated for $2 \mathrm{D}$ and $3 \mathrm{D}$ area sources, and dose from beta and photon radiation can be calculated from point sources. The area over which the dose is averaged and the depth at which dose is calculated must be consistent with the requirements given in 10 CFR 835.205. To demonstrate compliance with 10 CFR 835, dose equivalent must be calculated at a depth of $0.007 \mathrm{~cm}$ or a technically equivalent density thickness of skin. Although the density of soft tissue is $1.06 \mathrm{~g} / \mathrm{cm}^{3}$, and a 
depth of 0.007 in soft tissue would thus correspond to a density thickness slightly greater than $7 \mathrm{mg} / \mathrm{cm}^{2}$, skin dose may be assessed with VARSKIN 3 assuming a density thickness of $7 \mathrm{mg} / \mathrm{cm}^{2}$. Chapter 6 of the DOE Radiation Protection Programs Guide DOE G 441.1-1C Radiation Protection Programs Guide (DOE 2008a) indicates that both values are technically equivalent for the purpose of demonstrating compliance with 10CFR835. The final assessment of equivalent dose to the skin or extremity and method of recording dose must be consistent with requirements given in 10 CFR 835.205 based on the area irradiated.

Hanford contractors may perform skin dose assessments, request assistance from HEDP in the assessment, or request that HEDP perform the assessment. In all cases, the contractor must approve the final dose assigned to the individual and entered into the record. Contractor dose assessments are reviewed by an HEDP Dosimetrist. All assessments are retained by the HRRP in the affected individual's personal exposure history file. When the irradiated area is $10 \mathrm{~cm}^{2}$ or greater, the assessed dose to the skin or extremity is entered into the REX database in a manner such that it will be added to the individual's calendar year and lifetime dose totals for either the skin or extremities in accordance with 10 CFR 835.205(b)(1) and (2). In the Hanford Radiological Records database, dose entered into the record as skin dose will also be counted toward extremity dose totals, but dose entered as extremity dose will not be counted toward skin dose totals (see Chapter 4). Hanford practices in these areas have been coordinated through and endorsed by the Hanford Personnel Dosimetry Advisory Committee (HPDAC).

\subsubsection{Alpha Contamination}

If alpha contamination is reported on the skin or clothing, the shallow dose equivalent from alpha radiation may be considered to be negligible (i.e., a 5-MeV alpha particle will penetrate tissue to a depth of approximately $3-4 \mathrm{mg} / \mathrm{cm}^{2}$, or less than the $7-\mathrm{mg} / \mathrm{cm}^{2}$ depth of regulatory concern.

\subsubsection{General Guidelines}

VARSKIN 3 calculates the operational quantity dose equivalent at a specified depth in tissue, averaged over a specified area. The results of one or more of these calculations are then used to "assess" equivalent dose to skin or extremity, using professional judgment and following the requirements in 10 CFR 835.205. One part of the "assessment" process is following the $\mathrm{H}=\mathrm{fD}$ rule in 835.205(b)(2) when applicable. When performing skin dose assessments, the following guidelines should be followed:

- The total density thickness of all clothing between the layer of contaminated clothing and the individual's skin should be considered in the dose calculation. The thickness of the piece of contaminated clothing should not be included in this total, unless it can be shown that the contamination rested on the outside of this piece of clothing. If the survey instrument count rate on the inside of the clothing is provided, this value should be used, which avoids using the thickness of the piece of contaminated clothing. 
- If two or more distinct (non-continuous) areas of contamination are present, calculations should be performed for each area. Each area should be evaluated separately and the highest shallow dose equivalent used to assess dose to the skin or extremity for the event being assessed.

- If more than one radionuclide is present, including daughter products, the shallow dose equivalent should be calculated for each radionuclide. The total shallow dose equivalent based on a summation of the shallow dose equivalent from all radionuclides, should then be used as the basis for assessing equivalent dose to the skin or extremity.

- If the assessed equivalent dose to the skin or extremity is greater than or equal to 15 rem, an evaluation should be conducted to ensure that there is no unrealistic conservatism in the calculated shallow equivalents used. Considerable professional judgment may be necessary, depending upon the particular circumstances of the incident.

- If the assessed equivalent dose to the skin or extremity is greater than or equal to 15 rem and the contaminant composition is unknown or in question, the contractor organization should obtain and analyze samples to identify the specific radionuclides involved. If samples are not available, other information may be used to determine the radionuclides such as work history or interviews with workers. If no positive radionuclide identification can be made, the radionuclides and percentages present in the contamination should be conservatively estimated.

- If the assessed equivalent dose to the skin or extremity is greater than or equal to $15 \mathrm{rem}$, and is due to contamination on personal or protective clothing and the clothing thickness is not accurately known, samples should be obtained and evaluated for a more accurate determination of density thickness and potential attenuation effect on calculated shallow dose equivalent. If possible a determination as to whether the contamination resided inside or outside the contaminated layer(s) of clothing should be made and taken into consideration in the calculations.

- If the assessed equivalent dose to the skin or extremity is greater than or equal to $15 \mathrm{rem}$, and is due to contamination on the skin, the thickness of clothing covering the contaminated area of skin should be accurately determined, as well as the distance of the clothing from the skin. An assessment should be made of the potential effects of backscatter from the clothing on the accuracy of the VARSKIN 3 calculated shallow dose equivalent and corrections made if appropriate. Refer to Section 8.2.4.

- If the assessed equivalent dose to the skin or extremity is greater than or equal to 15 rem, an attempt should be made to obtain the instrument used for the contamination survey, verify its calibration and characterize its response to the source isotope and geometry if they are known.

- The area over which average shallow dose equivalent is calculated and the depth at which dose is calculated must be consistent with the 
requirements given in 10 CFR 835.205. A density thickness of $7 \mathrm{mg} / \mathrm{cm}^{2}$ may be assumed as the depth of skin when performing dose calculations.

- When calculating shallow dose equivalent from hot particles, the dimensions and composition of the particle, as well as the radionuclide(s) should be known. In the case of low energy beta emitters such as ${ }^{60} \mathrm{Co}$, self attenuation within the particle may be significant such that the primary dose to skin and nearby organs is from gamma radiation. Hot particles should be modeled using one of the 3D geometries available in VARSKIN 3 to take account of the shelf shielding within the particle.

\subsubsection{Validation of VARSKIN 3}

Development of the VARSKIN 3 computer code was commissioned by the NRC. VARSKIN 3 has undergone V\&V testing by its author (Durham 2006b) as well as by the NRC (Sherbini et al. 2008) and has been generally accepted for use by the NRC. The NRC testing expanded upon previous testing performed by the code's author and third parties to include all the source geometries that are programmed in the code over a wide range of beta energies and skin depths. The NRC tested the code by comparing VARSKIN calculated doses with doses for similar geometries calculated using MCNP5. Beta emitters with end point energies from $0.3 \mathrm{MeV}$ up to $2.3 \mathrm{MeV}$ were tested. The NRC study found excellent agreement (within a few percent for point and disk sources and within $20 \%$ for other sources with the exception of a few cases at the low end of the beta energies tested). The NRC concluded that VARSKIN is sufficiently accurate for calculation of skin dose arising from skin contamination, and that the uncertainties arising from use of VARSKIN are small compared to uncertainties in the input data used. Skin dose assessments are often characterized by significant uncertainty in the size, shape and density of the contaminant, the depth of the sensitive layer of the skin at the location of the contamination, the duration of the exposure, and the area of skin irradiated. The area irradiated is particularly uncertain when the contamination is on protective clothing and the source is likely moving over various areas of the skin during the exposure period.

In addition to the above described testing, VARSKIN 3 has been tested by HEDP for typical applications at Hanford and accepted for use at Hanford. ${ }^{\text {(a) }}$ In HEDP testing, the dose results obtained with VARSKIN 3 were compared with dose results obtained by other methods. Skin dose conversion factors published in the peer reviewed literature for selected radionuclides are shown are shown in Table 8.4 together with values obtained from VARSKIN 3 calculations. The values in Table 8.4 are for area contamination. The conversion factors obtained from the peer reviewed literature were calculated using both point kernel integration and Monte Carlo methods. The data show good agreement between VARSKIN 3 values and the literature values. In nearly all cases, the VARSKIN 3 values are bracketed by the literature values. Conversion factors for point sources are compared in Table 8.5. Good agreement is also demonstrated for point sources.

(a) B.A. Rathbone, "Validation and Verification of VARSKIN 3" May 11, 2009, memo to HEDP file. VARSKIN 3, Version 3.0.1 Software Application Document 4/28/2009 (HEDP document) 
Table 8.4 Comparison of Skin Dose Conversion Factors for Area Contamination

\begin{tabular}{|c|c|c|c|c|c|c|c|c|c|}
\hline \multirow[b]{2}{*}{ Nuclide } & \multirow[b]{2}{*}{$\begin{array}{l}\beta_{\text {avg }} \\
(\mathrm{keV})\end{array}$} & \multirow[b]{2}{*}{$\begin{array}{l}\beta_{\max } \\
(\mathrm{keV})\end{array}$} & \multicolumn{6}{|c|}{ Dose Rate $\quad\left(\mathrm{mrem} / \mathrm{h}\right.$ per $\left.\mathrm{dpm} / \mathrm{cm}^{2}\right)$} & \multirow{2}{*}{$\begin{array}{l}\mathrm{dpm}-\mathrm{h} \text { required for } \\
100 \text { mrem dose to } \\
\text { maximally exposed } \\
1 \mathrm{~cm}^{2} \text { based on } \\
\text { largest dose } \\
\text { conversion factor. }\end{array}$} \\
\hline & & & $\begin{array}{l}\text { Kocher and } \\
\text { Eckerman, } \\
1987^{\mathrm{a}}\end{array}$ & $\begin{array}{l}\text { Faw, } \\
1992^{b}\end{array}$ & $\begin{array}{c}\text { Rohloff and } \\
\text { Heinzelmann, } \\
1986^{c}\end{array}$ & $\begin{array}{l}\text { Cross } \\
\text { et. al., } \\
1992^{d}\end{array}$ & $\begin{array}{l}\text { VARSKIN } 3 \\
\text { Durham, } \\
2006^{\mathrm{e}}\end{array}$ & Maximum & \\
\hline C-14 & 49 & 158 & 5.51E-04 & 4.75E-04 & 5.57E-04 & $5.23 \mathrm{E}-04$ & 5.04E-04 & 5.57E-04 & $2.69 \mathrm{E}+06$ \\
\hline P-32 & 694 & 1709 & $3.99 \mathrm{E}-03$ & $2.64 \mathrm{E}-03$ & $3.02 \mathrm{E}-03$ & $2.91 \mathrm{E}-03$ & $2.99 \mathrm{E}-03$ & $3.99 \mathrm{E}-03$ & $3.76 \mathrm{E}+05$ \\
\hline S-35 & 48 & 167 & $5.89 \mathrm{E}-04$ & $5.13 \mathrm{E}-04$ & $5.60 \mathrm{E}-04$ & $5.62 \mathrm{E}-04$ & $5.46 \mathrm{E}-04$ & 5.89E-04 & $2.55 \mathrm{E}+06$ \\
\hline $\mathrm{Cl}-36$ & 252 & 714 & $3.23 \mathrm{E}-03$ & $n / a$ & $\mathrm{n} / \mathrm{a}$ & $2.81 \mathrm{E}-03$ & $2.66 \mathrm{E}-03$ & $3.23 \mathrm{E}-03$ & $4.64 \mathrm{E}+05$ \\
\hline Co-60 & 94 & 1478 & $1.88 \mathrm{E}-03$ & $1.50 \mathrm{E}-03$ & $1.46 \mathrm{E}-03$ & $1.73 \mathrm{E}-03$ & 1.70E-03 & $1.88 \mathrm{E}-03$ & $7.97 \mathrm{E}+05$ \\
\hline Sr-90 & 200 & 544 & 3.04E-03 & $2.22 \mathrm{E}-03$ & $2.38 \mathrm{E}-03$ & $2.46 \mathrm{E}-03$ & $2.46 \mathrm{E}-03$ & $3.04 \mathrm{E}-03$ & $4.93 \mathrm{E}+05$ \\
\hline Y-90 & 931 & 2245 & $3.99 \mathrm{E}-03$ & $2.68 \mathrm{E}-03$ & $3.05 \mathrm{E}-03$ & $2.96 \mathrm{E}-03$ & $3.09 \mathrm{E}-03$ & $3.99 \mathrm{E}-03$ & $3.76 \mathrm{E}+05$ \\
\hline Mo-99 & 398 & 1215 & $3.61 \mathrm{E}-03$ & $2.45 \mathrm{E}-03$ & $\mathrm{n} / \mathrm{a}$ & $\mathrm{n} / \mathrm{a}$ & $2.68 \mathrm{E}-03$ & $3.61 \mathrm{E}-03$ & $4.16 \mathrm{E}+05$ \\
\hline Tc-99 & 85 & 295 & $1.63 \mathrm{E}-03$ & $1.63 \mathrm{E}-03$ & $\mathrm{n} / \mathrm{a}$ & $1.70 \mathrm{E}-03$ & $1.88 \mathrm{E}-03$ & $1.88 \mathrm{E}-03$ & $8.00 \mathrm{E}+05$ \\
\hline Rh-106 & 1415 & 3541 & $4.18 \mathrm{E}-03$ & $\mathrm{n} / \mathrm{a}$ & $3.16 \mathrm{E}-03$ & $3.13 \mathrm{E}-03$ & $3.33 \mathrm{E}-03$ & $4.18 \mathrm{E}-03$ & $3.59 \mathrm{E}+05$ \\
\hline $\mathrm{I}-131$ & 180 & 810 & $2.85 \mathrm{E}-03$ & $2.15 \mathrm{E}-03$ & $2.24 \mathrm{E}-03$ & $2.37 \mathrm{E}-03$ & $2.36 \mathrm{E}-03$ & $2.85 \mathrm{E}-03$ & $5.26 \mathrm{E}+05$ \\
\hline Cs-134 & 152 & 1453 & $2.28 \mathrm{E}-03$ & $1.63 \mathrm{E}-03$ & $\mathrm{n} / \mathrm{a}$ & $1.78 \mathrm{E}-03$ & $1.79 \mathrm{E}-03$ & $2.28 \mathrm{E}-03$ & $6.58 \mathrm{E}+05$ \\
\hline Cs-137 & 195 & 1167 & $2.66 \mathrm{E}-03$ & $2.11 \mathrm{E}-03$ & $n / a$ & $2.56 \mathrm{E}-03$ & 2.31E-03 & $2.66 \mathrm{E}-03$ & $5.64 \mathrm{E}+05$ \\
\hline Ce-144 & 81 & 320 & $1.69 \mathrm{E}-03$ & $n / a$ & $n / a$ & $1.50 \mathrm{E}-03$ & $1.53 \mathrm{E}-03$ & 1.69E-03 & $8.87 \mathrm{E}+05$ \\
\hline Eu-154 & 228 & 1850 & $3.42 \mathrm{E}-03$ & $2.76 \mathrm{E}-03$ & $n / a$ & $3.29 \mathrm{E}-03$ & $2.83 \mathrm{E}-03$ & 3.42E-03 & $4.39 \mathrm{E}+05$ \\
\hline \multirow[t]{3}{*}{ TI-204 } & 267 & 765 & 3.23E-03 & $\mathrm{n} / \mathrm{a}$ & $2.36 \mathrm{E}-03$ & $2.25 \mathrm{E}-03$ & $2.37 \mathrm{E}-03$ & 3.23E-03 & $4.64 \mathrm{E}+05$ \\
\hline & & & & & & & & & $\begin{array}{l}\text { Minimum dpm-h } \\
\text { required for } 100 \\
\text { mrem }^{f}\end{array}$ \\
\hline & & & & & & & & & $3.59 \mathrm{E}+05$ \\
\hline & & & & & & & & & \\
\hline a & \multicolumn{9}{|c|}{$\begin{array}{l}\text { Kocher, DC, KF Eckerman. 1987. Electron Dose-Rate Conversion Factors for External Exposure of the Skin from Uniformly Deposited } \\
\text { Activity on the Body Surface. Health Physics } 53 \text { (2) 135-141. Data are adapted from Table } 1 \text {. Numeric integration of Berger point kernel. } \\
\text { Calculation of dose at a depth of } 7 \mathrm{mg} / \mathrm{cm}^{2} \text { in water from an infinite plane source on a water/water boundary. Dose includes contribution } \\
\text { from beta particles backscattered from a water. No correction for air vs. water backscatter. Potential overestimate of skin dose by as much } \\
\text { as } 35 \% \text {. }\end{array}$} \\
\hline b & \multicolumn{9}{|c|}{$\begin{array}{l}\text { Faw, RE. 1992. Absorbed Doses to Skin from Radionuclide Sources on the Body Surface. Health Physics } 63(4): 443-448 \text {. Data are } \\
\text { adapted from Table } 5 \text { lower bound values (without air scatter contribution). Monte Carlo calculation of dose in tissue at a depth of 5-10 } \\
\mathrm{mg} / \mathrm{cm}^{2} \text { from an infinite plane source on a tissue/vacuum boundary. The values from Table } 5 \text { that do not include air scatter are } \\
\text { recommended as more accurate for skin contamination of finite area. }\end{array}$} \\
\hline c & \multicolumn{9}{|c|}{$\begin{array}{l}\text { Rohloff, F. Heinzelmann, M. 1986. Calculation of Dose Rates for Skin Contamination by Beta Radiation. Radiation Protection Dosimetry } \\
\text { 14(4): } 279-287 \text {. Data are adapted from Table } 1 \text {. Monte Carlo calculation of dose at depth of } 0.07 \mathrm{~mm} \text { in water from infinite plane source on } \\
\text { water/vacuum boundary. Dose does not include contribution from backscattered beta particles. Considered more representative of dose to } \\
\text { skin contamination of finite area than values based on full air reflection from infinite source. }\end{array}$} \\
\hline d & \multicolumn{9}{|c|}{$\begin{array}{l}\text { Cross, WG, NO Freedman, PY Wong. 1992. Beta Ray Dose Distributions form Skin Contamination. Radiation Protection Dosimetry } \\
\text { 40(3):149-168. Data adapted from Table 5. Monte Carlo calculation of dose to a point at a depth of } 0.07 \mathrm{~mm} \text { on the axis of a circular } 100 \\
\mathrm{~cm}^{2} \text { plane isotropic source on an air-water boundary. Dose includes contribution from air backscattered beta particles. }\end{array}$} \\
\hline e & \multicolumn{9}{|c|}{$\begin{array}{l}\text { Durham, JS. 2006. VARSKIN 3: A Computer Code for Assessing Skin Dose from Skin Contamination NUREG/CR-6918. U.S. Nuclear } \\
\text { Regulatory Comission. Calculation based on numeric integration of Berger point kernel for water, but includes correction for differences } \\
\text { between backscatter from water vs. backscatter from air. The values in this column were calculated for a } 100 \mathrm{~cm}^{2} \text { contamination area with } \\
\text { dose averaged over the maximally exposed } 1 \mathrm{~cm}^{2} \text {. With the backscatter correction applied by VARSKIN 3, the calculated dose in theory } \\
\text { includes contribution from air backscattered beta particles. }\end{array}$} \\
\hline$f$ & \multicolumn{9}{|c|}{$\begin{array}{l}\text { The dpm in "dpm-h" refers to the activity under a } 15 \mathrm{~cm}^{2} \text { pancake probe which is what is typically recorded on skin contamination forms } \\
\text { based on observed } \mathrm{cpm} \times 10 \text {. The term "dpm-h" refers to the } \mathrm{dpm} \text { measured in a } 15 \mathrm{~cm}^{2} \text { area } \mathrm{x} \text { the number of exposure hours. }\end{array}$} \\
\hline
\end{tabular}


Table 8.5 Comparison of Skin Dose Conversion Factors for Point Source Contamination

\begin{tabular}{|c|c|c|c|c|c|c|}
\hline \multirow[b]{2}{*}{ Nuclide } & \multirow{2}{*}{$\begin{array}{c}\beta_{\text {avg }} \\
(\mathrm{keV})\end{array}$} & \multirow{2}{*}{$\begin{array}{l}\beta_{\max } \\
(\mathrm{keV})\end{array}$} & \multicolumn{3}{|c|}{ Dose Rate $\quad(\mathrm{mrem} / \mathrm{dpm}-\mathrm{h})$} & \multirow{2}{*}{$\begin{array}{l}\text { dpm-h required for } 100 \\
\text { mrem dose to maximally } \\
\text { exposed } 1 \mathrm{~cm}^{2} \text { based on } \\
\text { largest dose conversion } \\
\text { factor. }\end{array}$} \\
\hline & & & $\begin{array}{l}\text { Cross } \\
\text { et. al., } \\
1992^{a}\end{array}$ & $\begin{array}{l}\text { VARSKIN } 3 \\
\text { Durham, } \\
2006^{b}\end{array}$ & Maximum & \\
\hline C-14 & 49 & 158 & 4.83E-04 & 5.00E-04 & 5.00E-04 & $2.00 \mathrm{E}+05$ \\
\hline P-32 & 694 & 1709 & $2.88 \mathrm{E}-03$ & 2.99E-03 & 2.99E-03 & $3.35 \mathrm{E}+04$ \\
\hline S-35 & 48 & 167 & 5.17E-04 & $5.45 \mathrm{E}-04$ & 5.45E-04 & $1.83 \mathrm{E}+05$ \\
\hline $\mathrm{Cl}-36$ & 252 & 714 & 2.63E-03 & 2.67E-03 & 2.67E-03 & $3.75 \mathrm{E}+04$ \\
\hline Co-60 & 94 & 1478 & 1.56E-03 & 1.69E-03 & 1.69E-03 & $5.90 \mathrm{E}+04$ \\
\hline Sr-90 & 200 & 544 & 2.30E-03 & $2.46 \mathrm{E}-03$ & $2.46 \mathrm{E}-03$ & $4.06 \mathrm{E}+04$ \\
\hline Y-90 & 931 & 2245 & 2.93E-03 & 3.05E-03 & 3.05E-03 & $3.27 \mathrm{E}+04$ \\
\hline Mo-99 & 398 & 1215 & $2.56 \mathrm{E}-03$ & $2.68 \mathrm{E}-03$ & $2.68 \mathrm{E}-03$ & $3.72 \mathrm{E}+04$ \\
\hline Tc-99 & 85 & 295 & 1.54E-03 & 1.87E-03 & 1.87E-03 & $5.35 \mathrm{E}+04$ \\
\hline Rh-106 & 1415 & 3541 & 3.08E-03 & 3.14E-03 & 3.14E-03 & $3.19 \mathrm{E}+04$ \\
\hline $\mathrm{I}-131$ & 180 & 810 & $2.20 \mathrm{E}-03$ & $2.36 \mathrm{E}-03$ & 2.36E-03 & $4.23 \mathrm{E}+04$ \\
\hline Cs-134 & 152 & 1453 & 1.67E-03 & 1.79E-03 & 1.79E-03 & $5.59 \mathrm{E}+04$ \\
\hline Cs-137 & 195 & 1167 & $2.39 \mathrm{E}-03$ & $2.30 \mathrm{E}-03$ & 2.39E-03 & $4.19 \mathrm{E}+04$ \\
\hline Ce-144 & 81 & 320 & 1.36E-03 & 1.52E-03 & 1.52E-03 & $6.59 \mathrm{E}+04$ \\
\hline Eu-154 & 228 & 1850 & 3.07E-03 & $2.83 \mathrm{E}-03$ & 3.07E-03 & $3.26 \mathrm{E}+04$ \\
\hline \multirow[t]{5}{*}{ TI-204 } & 267 & 765 & $2.24 \mathrm{E}-03$ & 2.37E-03 & 2.37E-03 & $4.21 \mathrm{E}+04$ \\
\hline & & & & & & $\begin{array}{l}\text { Minimum dpm-h required } \\
\text { for } 100 \text { mrem }\end{array}$ \\
\hline & & & & & & $3.19 \mathrm{E}+04$ \\
\hline & & & & & & \\
\hline & & & & & & \\
\hline a & \multicolumn{6}{|c|}{$\begin{array}{l}\text { Cross, WG, NO Freedman, PY Wong. 1992. Beta Ray Dose Distributions from Skin } \\
\text { Contamination. Radiation Protection Dosimetry 40(3):149-168. Data adapted from Table } 4 . \\
\text { Monte Carlo calculation of dose to a point at a depth of } 0.07 \mathrm{~mm} \text { on the axis of a circular } 1 \\
\mathrm{~cm}^{2} \text { plane isotropic source on an air-water boundary. Dose includes contribution from beta } \\
\text { particles backscattered in air. }\end{array}$} \\
\hline b & \multicolumn{6}{|c|}{$\begin{array}{l}\text { Durham, JS. 2006. VARSKIN 3: A Computer Code for Assessing Skin Dose from Skin } \\
\text { Contamination NUREG/CR-6918. U.S. Nuclear Regulatory Comission. Calculation based } \\
\text { on numeric integration of Berger point kernel for water, but includes correction for differences } \\
\text { between backscatter from water vs. backscatter from air. The values in this column were } \\
\text { calculated for a point source with dose averaged over the maximally exposed } 1 \mathrm{~cm}^{2} \text {. With } \\
\text { the backscatter correction applied by VARSKIN 3, the calculated dose in theory includes } \\
\text { contribution from air backscattered beta particles. }\end{array}$} \\
\hline
\end{tabular}




\subsubsection{Considerations of the Air/Tissue Interface When Using VARSKIN 3}

The Berger point kernel used for beta dose calculations in VARSKIN (Berger 1971) calculates dose from a point source in an infinite water medium.

Backscatter of beta particles in water contributes to dose at the point of interest. Without correction, use of this kernel will over estimate dose to tissue from contamination on the skin because of the differences in density between air vs. water as a backscatter medium. VARSKIN 3 applies a backscatter correction to account for this difference and as a result provides reasonably accurate dose results for surface contamination on uncovered areas of skin.

Various authors have investigated the influence of the air-tissue interface on beta dose to the skin from thin layer contamination on the skin (Kocher and Eckerman 1987; Faw 1982; Rohloff and Heinzelmann 1986; Cross et al. 1992). Because an electron in air travels 844 times farther than one in water before making another collision (Crawford et al. 1991), the area of skin irradiated from electrons backscattered from air is larger than the area irradiated from electrons backscattered from water for point or small area sources. For moderate and higher energy beta particles as exemplified by ${ }^{36} \mathrm{Cl}$, (710 keV max, $279 \mathrm{keV}$ avg.) a significant dose component is present at radial distances appreciably beyond the $1 \mathrm{~cm}^{2}$ area typically used in skin dose assessment. When water is the backscatter medium, dose is concentrated more closely to the source. At small radii from the source, the dose from air backscatter is smaller than from water. At larger radii, the dose from air backscatter exceeds that from water. When dose to skin from a ${ }^{36} \mathrm{Cl}$ point source is averaged over $1 \mathrm{~cm}^{2}$ of skin, the dose with water as a backscatter medium is $46 \%$ higher than with air as the backscatter medium. When dose to skin from a ${ }^{36} \mathrm{Cl}$ point source is averaged over $100 \mathrm{~cm}^{2}$ of skin, the dose with water as a backscatter medium is 33\% higher than with air as the backscatter medium. Similar relationships hold for area sources that are small in diameter relative to the range of the beta particles in air. However if the source is considered to be an infinite plane (i.e. source diameter more than twice the range of beta particles in air) on an air/water or water/water boundary, then the dose resulting from air vs. water backscatter is the same (Rohloff and Heinzelmann 1986).

The effects of differing backscatter media should be taken into consideration when assessing dose to skin from contamination on the skin. Specifically, based on the above information, it may be inferred that since VARSKIN 3 calculations for point sources and 2D disk sources assume backscatter from air, they may underestimate dose when backscatter comes from higher density materials such as protective clothing. If the protective clothing is in close contact with the skin and is thicker than the range of the maximum energy beta particle emitted from the source, the VARSKIN 3 calculated dose to a $1 \mathrm{~cm}^{2}$ area or $100 \mathrm{~cm}^{2}$ area from a point or small area source may potentially be only $68 \%$ or $75 \%$ of the true dose respectively, (for moderate and higher energy beta emitters). This should be taken into consideration when large doses (e.g. $\geq 15$ rem) are being calculated and higher accuracy may be needed in the calculation. 


\subsection{Recommended Limits for Hot Particles}

The DOE radiological control standard DOE-STD-1098-2008 Radiological Control (DOE 2008b) recommends that the site specific radiological control manual define hot particles, such as those capable of producing an equivalent dose to the skin greater than 100 millirem in one hour, specific to facility operations and source terms. At Hanford, hot particles are defined as solid particles less than $1 \mathrm{~mm}$ in diameter, that are not water soluble and have an activity of at least $10 \mu \mathrm{Ci}$. This definition was endorsed by the HPDAC ${ }^{(a)}$ in 1994. The Hanford definition corresponds to particles capable of producing an equivalent dose to skin on the order of several tens of rem on one hour (61.2 rem for ${ }^{90} \mathrm{Sr} /{ }^{90} \mathrm{Y}$ ). The rationale for this definition was based on the following:

- NCRP Report No. 106, Limit for Exposure to 'Hot Particles' on the Skin, contained a recommendation that exposures to hot particles be limited to 75 $\mu$ Ci-h (NCRP 1989).

- The maximum duration for a normal entry into a surface contamination area is 4 hours.

- A limit of $40 \mu \mathrm{Ci}$-h (i.e., $10 \mu \mathrm{Ci}$ x 4 hours) is approximately half of the NCRP recommended limit.

NCRP Report No. 106 has been superseded by NCRP Report No. 130, Biological Effects and Exposure Limits for "Hot Particles" (NCRP 1999). NCRP Report No. 130 makes the following recommendations:

Skin: $\quad$ The dose to skin at a depth of $70 \mu \mathrm{m}$ from hot particles on skin (including ear), hair or clothing be limited to no more than 0.5 Gy (50 rad) averaged over the most highly exposed $10 \mathrm{~cm}^{2}$ of skin.

Observation of the exposed area of skin for four to six weeks be initiated whenever the dose evaluated at a depth of $70 \mu \mathrm{m}$ exceeds $0.1 \mathrm{~Gy}$ averaged over the most highly exposed $10 \mathrm{~cm}^{2}$ of skin.

Eye: $\quad$ The dose at a depth of $70 \mu \mathrm{m}$ to any ocular tissue from hot particles be limited to $5 \mathrm{~Gy}$ (500 rad) averaged over the most highly exposed $1 \mathrm{~cm}^{2}$ of ocular tissue.

Observation of the eye for four to six weeks be initiated whenever the dose, evaluated at a depth of $70 \mu \mathrm{m}$, exceeds 1 Gy averaged over the most highly exposed $1 \mathrm{~cm}^{2}$ of ocular tissue.

The report also makes recommendations for exposure limitation of the respiratory tract and gastrointestinal tract.

(a) Fix, J. J. "Minutes of Hanford Personnel Dosimetry Advisory Committee Meeting on December 14, 1994.” (A copy is available in the Hanford Radiation Protection Historical Files, Pacific Northwest National Laboratory, Richland, Washington.) 
The recommended limits for the skin and eye are intended to minimize the occurrence of adverse deterministic effects such as lesions that result in the breakdown of the barrier function of the skin. For low energy beta particles (< $0.5 \mathrm{MeV}$ max) the lesion of concern is acute epidermal necrosis. For intermediate ( $\geq 0.5$ to $1.5 \mathrm{MeV}$ ) and high energy beta particles $(>1.5 \mathrm{MeV})$ the lesion of concern is acute ulceration. Because of the involvement of dermal tissue, such lesions when established tend to have longer duration than is associated with acute epidermal necrosis (NCRP 1999).

Although expressed in different units, the new NCRP 130 limits are generally consistent with the previous NCRP 106 limits. Using the VARSKIN 3 code (Durham 2006), the NCRP 106 limit of $75 \mu \mathrm{Ci}$-h results in a skin dose of $46 \mathrm{rad}$ for a ${ }^{90} \mathrm{Sr} /{ }^{90} \mathrm{Y}$ point source (dimensionless) when the dose is averaged over 10 $\mathrm{cm}^{2}$. When averaged over $1 \mathrm{~cm}^{2}$, the dose is $459 \mathrm{rad}$. For a ${ }^{60}$ Co point source (dimensionless), the calculated skin dose is about 32 rad when averaged over 10 $\mathrm{cm}^{2}$. When averaged over $1 \mathrm{~cm}^{2}$, the calculated dose is $310 \mathrm{rad}$.

\subsubsection{Assessment of Dose from Hot Particles}

For assessment of equivalent dose to the skin or extremity from discrete betagamma emitting particles on the skin, VARSKIN 3 should be used to calculate the required operational quantities. For skin dose from discrete particles, the "area irradiated" may be considered to be less than $10 \mathrm{~cm}^{2}$. When the area irradiated is less than $10 \mathrm{~cm}^{2}$, the assessed dose is not added to calendar year totals for the purpose of demonstrating compliance with 10 CFR 835.202(a)(4). The general guidelines in Section 8.2.2 should be followed when assessing skin dose from discrete particles, including particles meeting the definition of hot particles.

The Pancake GM action level in Table 8.1 for "Contamination Area < Probe Area" is sufficient to identify discrete particles capable of producing an equivalent dose to the skin of 100 mrem or more after two hours on the skin. The equivalent criteria of $\mathbf{5 0 0 0} \mathbf{~ c p m}$-h may be used for identifying discrete particle exposures of any duration that potentially exceed 100 mrem. Although ionization chamber (RO-2 and RO-3B) responses to very small sources of radiation simulating hot particles have been measured and are presented in Table 8.6, the readings that would be obtained for discrete particles corresponding to only 100 mrem skin dose are below the measurable range for these instruments. The data in Table 8.6 were developed primarily for use in assessments of skin dose from high activity particles (i.e. hot particles) where the readings on a Pancake GM are off scale.

It should be kept in mind that for low energy beta emitters such as ${ }^{60} \mathrm{Co}$, a significant fraction of the beta radiation may be attenuated within the particle depending on particle size. For these particles, most of the SDE and all of the DDE may be due to gamma radiation even when in direct contact with the skin.

It is also important to keep in mind that gamma emitting hot particles have the potential to produce localized dose to tissues at depths greater than $1 \mathrm{~cm}$, potentially requiring an assessment of effective dose, $\mathrm{E}$, to demonstrate compliance with 10 CFR 835.202(a)(1) and (2). Such assessments should be 
performed when the deep dose equivalent potentially exceeds 100 mrem. Table 8.7 shows the calculated DDE from point sources as a function of photon energy (Xu 2005). In cases involving a single hot particle, effective dose, E, will generally be less than $1 \%$ of the DDE.

Table 8.6. Measured Eberline Model RO-2 and RO-3B Ionization Chamber Response and Calculated Dose for Point $\left(<2 \mathrm{~mm}^{2}\right)$ Radiation Sources ${ }^{\text {(a) }}$

\begin{tabular}{|c|c|c|c|c|c|}
\hline Nuclide & $\begin{array}{c}\text { Activity } \\
(\mu \mathrm{Ci})^{\mathrm{a}}\end{array}$ & $\begin{array}{l}\text { Reading } \\
(\mathrm{mrad} / \mathrm{h})^{\mathrm{b}}\end{array}$ & $\begin{array}{l}\text { Dose Rate } \\
(\mathrm{mrad} / \mathrm{h})^{\mathrm{c}}\end{array}$ & $\begin{array}{c}\text { Reading/Dose Rate } \\
\left([\mathrm{mrad} / \mathrm{h}]_{\mathrm{CP}} /\right. \\
\left.[\mathrm{mrad} / \mathrm{h}]_{\text {skin }}\right)\end{array}$ & $\begin{array}{c}\text { Dose Rate/Reading } \\
\left([\mathrm{mrad} / \mathrm{h}]_{\text {skin }} /\right. \\
\left.[\mathrm{mrad} / \mathrm{h}]_{\mathrm{CP}}\right)^{\mathrm{d}}\end{array}$ \\
\hline \multicolumn{6}{|c|}{ Eberline Model RO-2 } \\
\hline${ }^{90} \mathrm{Sr} /{ }^{90} \mathrm{Y}$ & 7.9 & Avg $=198$ & 92,500 & $2.1 \times 10^{-3}$ & 480 \\
\hline${ }^{137} \mathrm{Cs}$ & 7.3 & Avg $=192$ & 42,000 & $4.6 \times 10^{-3}$ & 219 \\
\hline \multicolumn{6}{|c|}{ Eberline Model RO-3B } \\
\hline${ }^{90} \mathrm{Sr} /{ }^{90} \mathrm{Y}$ & 7.9 & Avg $=108$ & 92,500 & $1.2 \times 10^{-3}$ & 860 \\
\hline${ }^{137} \mathrm{Cs}$ & 7.3 & Avg $=110$ & 42,000 & $2.6 \times 10^{-3}$ & 380 \\
\hline \multicolumn{6}{|c|}{$\begin{array}{l}\text { a. Activity decay corrected to time of measurement. } \\
\text { b. Average reading for } 5 \text { to } 10 \text { measurements. Data for source to instrument window distance of } 0.5 \mathrm{in} \text {. } \\
\text { c. VARSKIN MOD2 calculated dose to } 1 \mathrm{~cm}^{2} \text {. } \\
\text { d. The most conservative case for the RO-2 and RO-3B is }{ }^{90} \mathrm{Sr} /{ }^{90} \mathrm{Y} \text { and results in calculated skin dose } \\
\text { assessment action levels of } 0.1 \text { and } 0.06 \mathrm{mrad} / \mathrm{h} \text { for the RO-2 and RO-3B, respectively. }\end{array}$} \\
\hline
\end{tabular}

Table 8.7. DDE for Photon Point Sources on Skin

\begin{tabular}{|c|c|c|}
\hline \multirow{2}{*}{ Energy (MeV) } & \multicolumn{2}{|c|}{$\mathrm{DDE}^{\mathrm{a}, \mathrm{b}}$} \\
\hline & $\mu \mathrm{Sv} \mathrm{h}^{-1} \mathrm{MBq}^{-1}$ & $\mu \mathrm{rem} \mathrm{h}^{-1} \mu \mathrm{Ci}^{-1}$ \\
\hline 0.1 & 99.17 & 366.94 \\
\hline 0.2 & 242.43 & 896.97 \\
\hline 0.4 & 540.65 & 2000.41 \\
\hline 0.6 & 813.30 & 3009.23 \\
\hline${ }^{137} \mathrm{Cs}$ & 763.81 & 2826.09 \\
\hline 0.8 & 1097.80 & 4061.86 \\
\hline 1.0 & 1319.43 & 4881.88 \\
\hline${ }^{60} \mathrm{Co}$ & 3148.97 & 11651.19 \\
\hline 1.5 & 1848.74 & 6840.33 \\
\hline 2.0 & 2288.36 & 8466.92 \\
\hline \multicolumn{3}{|c|}{$\begin{array}{l}\text { Assumes one photon per disintegration except for }{ }^{137} \mathrm{Cs}(0.85 \\
\text { photons/disintegration) and }{ }^{60} \mathrm{Co}(2 \text { photons per disintegration). } \\
\text { Adapted from Xu, } 2005\end{array}$} \\
\hline
\end{tabular}

(a) Letter from C. E. Upchurch to J. D. Frey, dated March 23, 1994, "Dose Rate Measurements of Simulated Hot Particles." Westinghouse Hanford Company. 
Based on the worst case data from Table 8.7, an $8.6 \mu \mathrm{Ci}$-h $\left(19.1 \times 10^{6} \mathrm{dpm}-\mathrm{h}\right)$ exposure from a ${ }^{60} \mathrm{Co}$ particle on the skin will produce 100 mrem DDE. Assuming a pancake GM probe efficiency of $10 \%$ for ${ }^{60} \mathrm{Co}^{(\mathrm{a})}$ this would equate to $1.91 \times 10^{6} \mathrm{cpm}-\mathrm{h}$. For a worst case exposure time of four hours, this is roughly equivalent to a $4.77 \times 10^{6} \mathrm{dpm}$ particle producing a direct reading of 477,000 cpm which is off-scale for most hand-held GM instruments. Therefore, it may be concluded that for skin contaminations producing readings that are onscale with a hand held a pancake GM instrument, calculations of DDE and assessments of $\mathrm{E}$ are generally not required. (Notable exceptions would be exposures from discrete particles at multiple whole body locations or locations near tissues with large tissue weighting factors such as the testes.)

Calculations of E resulting from gamma emitting hot particles at 74 locations covering the entire body surface were performed using MCNP and tabulated for reference (Xu 2005). In addition, DDE was calculated for the same range of photon energies used in the calculations of E. The tabulated data show that DDE significantly over estimates $\mathrm{E}$ (by two orders of magnitude for the photon energies and locations tabulated). For most photon energies, the chest, abdomen and waist locations yielded the highest calculated value for E. For a $75 \mu \mathrm{Ci}$-h exposure from a ${ }^{137} \mathrm{Cs}$ point source located on the center front waist (highest $\mathrm{E}$ location for ${ }^{137} \mathrm{Cs}$ ), the calculated $\mathrm{E}$ is 0.625 mrem. By comparison, the calculated DDE is 212 mrem and the calculated SDE averaged over $1 \mathrm{~cm}^{2}$ of skin using VARSKIN 3 is 390 rem. In general, for beta-gamma emitting hot particles on the skin, dose to the skin (averaged over $1 \mathrm{~cm}^{2}$ ) will be very large, compared to the calculated DDE or E. It should be noted however, that the tabulated calculations (Xu 2005) did not explicitly consider hot particles in close proximity to tissues with large tissue weighting factors such as the male gonads. The general relationship between E, DDE and SDE illustrated above may not be applicable for hot particle exposure of the human testes. This is particularly true if the beta component is reduced by self attenuation within the particle or attenuation by protective clothing.

A user friendly software package called the EPRI EDE Calculator has also been developed which allows the user to input exposure data and calculate $\mathrm{E}$ automatically (Xu, et. al. 2006). This may be particularly useful where $\mathrm{E}$ from particles at multiple locations on the body must be assessed.

(a) PNNL-MA-562, "Radiation Protection Instrument Manual” Chapter 6.0 


\subsubsection{Recommended Action Levels for Hot Particle Control}

In accordance with NCRP recommendations, facilities that have hot particles in removable contamination should consider measures to limit personnel exposure to these particles. In 1994 measurements of simulated hot particles were made for the purpose of establishing facility action levels for hot particle control. ${ }^{\text {(a) }}$ Ionization chamber readings from point sources of radiation measuring less than $2 \mathrm{~mm}^{2}$ in area were made at several distances. The results are shown in Table 8.6. Based on the RO-2 and RO-3B response data in Table 8.6, uncorrected open window readings exceeding $100 \mathrm{mrad} / \mathrm{h}$ on removable contamination (e.g. on large area smears, adhesive pads or adhesive rollers, or on protective clothing or skin) may indicate the presence of hot particles.

In areas where the presence of hot particles has been confirmed, measures to limit the movement of hot particles and reduce the potential for worker exposure should be considered. ${ }^{\text {(b) }}$ One potential measure is to set up hot particle control areas to contain and limit the movement of particles. Another measure would be periodic surveys of protective clothing and exposed skin of workers dressed out inside hot particle control areas using ionization chamber type survey instruments to detect the presence of hot particles and limit potential exposures. DOE guidance on controls for hot particles is given in Article 348 of DOE-STD-10982008 Radiological Control (DOE 2008b).

(a) Letter from C. E. Upchurch to J. D. Frey, dated March 23, 1994, "Dose Rate Measurements of Simulated Hot Particles." Westinghouse Hanford Company.

(b) Fix, J. J. "Minutes of Hanford Personnel Dosimetry Advisory Committee Meeting on December 14, 1994.” (A copy is available in the Hanford Radiation Protection Historical Files, Pacific Northwest National Laboratory, Richland, Washington.) 
THIS PAGE INTENTIONALLY LEFT BLANK 


\subsection{References}

American National Standards Institute (ANSI). 1975. American National Standard Performance, Testing, and Procedural Specifications for Thermoluminescent Dosimetry (Environmental Applications). ANSI N545, American National Standards Institute, New York.

American National Standards Institute (ANSI). 1981. Dosimetry for Criticality Accidents. ANSI N13.3, American National Standards Institute, New York.

American National Standards Institute (ANSI). 1986. Criticality Accident Alarm System. ANSI N8.3, American National Standards Institute, New York.

American National Standards Institute (ANSI). 1993. Sampling Procedures and Tables for Inspection by Attributes. ANSI/ASQC Z1.4 - 1993, American National Standards Institute, New York.

Antonio, E. J. 1999. "External Radiation Surveillance” in Hanford Site Environmental Report for Calendar Year 1998. PNNL-12088.

Antonio, E. J. 2002. “External Radiation Surveillance” in Hanford Site Environmental Report for Calendar Year 2002. PNNL-14295.

Bartlett, D. T., P. J. Dimbylow, and T. M. Francis, 1990 “Calculated Backscatter from Phantoms for Photon Dosimeter Calibration” Radiation Protection Dosimetry, 32, 123-125: 1990

Baumgartner, W. V., A. W. Endres, and S. R. Reese. 1992. Quality Control Program for the Hanford External Dosimetry Thermoluminescent Processing System. PNL-8299, Pacific Northwest Laboratory, Richland, Washington.

Becker, K., J. S. Cheka, and M. Oberhofer. 1970. "Thermally Stimulated Exoelectron Emission, Thermoluminescence and Impurities in LiF and BeO," Health Phys. 18, 1970.

Berger, M. J. "Distribution of Absorbed Dose Around Point Sources of Electrons and Beta Particles in Water and Other Media.” Medical Internal Radiation Dose Committee, MIRD Pamphlet No. 7. Journal of Nuclear Medicine. Vol. 12, Supplement No. 5. pp. 5-22. 1971.

Bicron NE, 1999. Dose Algorithm: BGN-DOELAP User’s Manual, Publication No. ALGM-DN-U-0298-001, Pre-Release Date, March 11, 1999, Chapter 8, pages 14-17.

Brackenbush, L. W., W. V. Baumgartner, and J. J. Fix. 1991. Response of TLD-Albedo and Nuclear Track Dosimeters Exposed to Plutonium Sources. PNL-7881, Pacific Northwest Laboratory, Richland, Washington.

Brackenbush, L. W., G. W. R. Endres, J. M. Selby, E. J. Vallario. 1980. Personnel Neutron Dosimetry at Department of Energy Facilities. PNL-3213, Pacific Northwest Laboratory, Richland, Washington.

Bramson, P. E. 1962. Hanford Criticality Dosimeter. HW-71710, General Electric Company, Richland, Washington.

Cameron, J. R., N. Suntharalingham and G. N. Kenney. 1968 Thermoluminescent Dosimetry, University of Wisconsin Press

Casey, R., M. A. S. Jewett and R. A. Facey, 1982. "Effective Depth of Spermatogonia in Man. I: Measurement of 
Scrotal Thickness” Physics in Medicine and Biology 27(11):1349-1356

Charles, M. W. 1986. "Skin, Eye, and Testis: Current Exposure Problems and Recent Advances in Radiobiology” Journal of the Society for Radiation Protection 6(2):69-81.

Conrady MM, RK Berg, RI Scherpelz, and BA Rathbone. 2009. Neutron Measurements at Hanford's Plutonium Finishing Plant. PNNL-18649, Pacific Northwest National Laboratory, Richland, WA

Cortez, J. R., E. Storm, G. J. Littlejohn. 1977. Photon and Beta Response of a New Thermoluminescent Dosimeter Badge, LA-UR-77-3001, Los Alamos Scientific Laboratory, Los Alamos, New Mexico.

Crawford, O. H., J. E. Turner, R. N. Hamm and J. C. Ashley. 1991. "Effects of the Tissue-Air Interface in Calculations of the $\beta$-Particle Skin Dose at a Depth of $70 \mu \mathrm{m}$ ” Health Physics Vol. 61, No. 5, pp 641-645

Cross, W. G.,.1981. “Neutron Activation of Sodium in Phantoms and the Human Body” Health Physics Vol. 41 pp $105-121$.

Cross, W. G. and H. Ing. 1985. "Sodium Activation in the Human Body." Radiation Protection Dosimetry 10(14):265-276.

Cross, W. G., P. Y. Wong, and N. O. Freedman. 1991. "Dose Distributions for Normally Incident Beta Rays." Radiation Protection Dosimetry 35(2):77-91.

Cross, W. G., N. O. Freedman and P. Y. Wong. 1992. "Beta Ray Dose Distributions from Skin Contamination.” Radiation Protection Dosimetry 40(3):149-168.

Currie, L. A. 1968. "Limits for qualitative detection and quantitative determination" Analytical Chemistry 40: 586-593: 1968.

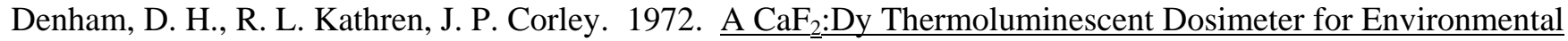
Monitoring. BNWL-SA-4191. Pacific Northwest Laboratory, Richland, Washington.

Doremus, S. W. and G. A. Higgins, 1994 "Pre-Irradiation Fade and Post-Irradiation Fade for LiF:Mg,Ti, TLD600 and TLD-700, as a Function of Time” Radiation Protection Dosimetry, Vol 54, No. 2, pp 119-125.

Douglas, J. A. 1981. “Applications of TL Materials in Neutron Dosimetry” in Applied Thermoluminescence Dosimetry, (M. Oberhofer and A. Scharmann, editors) Adam Hilger Ltd. 1981.

Durham, J. S. 1992. VARSKIN MOD2 and SADDE MOD2: Computer Codes for Assessing Skin Dose from Skin Contamination. NUREG/CR-5873, PNL-7913, Pacific Northwest Laboratory, Richland, Washington.

Durham, J. S. 2006. J. S. Durham, VARSKIN 3: A Computer Code for Assessing Skin Dose from Skin Contamination, NUREG/CR-6918 (2006).

Endres, A. W., L. W. Brackenbush, W. V. Baumgartner, J. J. Fix, and B.A. Rathbone. 1996. Response of Hanford Combination Neutron Dosimeter Exposed to Plutonium Sources. PNNL-10516, Pacific Northwest National Laboratory, Richland, Washington.

Facey, R. A. 1982. "Effective Depth of Spermatogonia in Man. II: Calculations for External High Energy Beta Rays” Physics in Medicine and Biology 27(11) 1357-1365. 
Faw, R. E. 1992. “Absorbed Doses to Skin from Radionuclide Sources on the Body Surface.” Health Physics 63(4):443-448.

Fix, J. J., J. M. Hobbs, P. L. Roberson, D. C. Haggard, K. L. Holbrook, M. R. Thorson, F. M. Cummings. 1982. Hanford Personnel Dosimeter Supporting Studies FY-1981. PNL-3736. Pacific Northwest Laboratory, Richland, Washington.

Fix, J. J., W.H. Wilson, and W. V. Baumgartner. 1996. Retrospective Assessment of Personnel Neutron Dosimetry for Workers at the Hanford Site. PNNL-11196, Pacific Northwest National Laboratory, Richland, Washington.

Fix, J. J., E. S. Gilbert, and W. V. Baumgartner. 1994. An Assessment of Bias and Uncertainty in Recorded Dose From External Sources of Radiation For Workers at the Hanford Site. PNL-10066, Pacific Northwest Laboratory, Richland, Washington.

Fix, J. J., G. W. R. Endres, F. M. Cummings, J. M. Aldrich, M. R. Thorson, R. L. Kathren. 1981. Hanford Personnel Dosimeter Supporting Studies FY-1980. PNL-3536, Pacific Northwest Laboratory, Richland, Washington.

Franklin, G. L. and Gonzalez, P. L. 1997 Beta Reduction Factors for Protective Clothing at the Oak Ridge National Laboratory, ORNL/CP-95027, Oak Ridge National Laboratory, 1997

Glenn, R. D., and P. E. Bramson. 1977. The Hanford Critical Radiation Dosimeter. PNL-2276, Pacific Northwest Laboratory, Richland, Washington.

Grosswendt. 1990. "Conversion Coefficients for Calibrating Individual Photon Dosimeters in Terms of Dose Equivalents in an ICRU Tissue Cube and PMMA Slabs." Radiation Protection Dosimetry 32(4):215-231.

Hankins, D. E. 1980. “Dosimetry of Criticality Accidents using Activations of the Blood and Hair” Health Physics Vol 38 pp 529 - 541.

Hankins, D. E. 1981. “Albedo Neutron Dosimeters Used as Area Monitors on Concrete Walls” Health Physics Vol 41 pp 543 - 546.

Harshaw/Filtrol TLD System 8800 Card Reader User's Manual for Use with Radiation Evaluation and Management System (REMS). 1988. Harshaw/Filtrol, Solon, Ohio.

Health Physics Society (HPS). 1993. American National Standard for Dosimetry - Personnel Dosimetry Performance - Criteria for Testing. HPS N13.11-1993, Health Physics Society, McLean, Virginia.

Health Physics Society (HPS). 1996a. American National Standard - Performance Testing of Extremity Dosimeters. HPS N13.32-1995, Health Physics Society, McLean, Virginia.

Health Physics Society (HPS). 1996b. American National Standard for Dosimetry , Environmental Dosimetry Performance - Criteria for Testing. HPS N13.29 (Draft), Health Physics Society, McLean, Virginia.

Health Physics Society (HPS). 2008. American National Standard - Performance Testing of Extremity Dosimeters. ANSI/HPS N13.32-2008, Health Physics Society, McLean, Virginia.

Health Physics Society (HPS). 2009. American National Standard for Dosimetry - Personnel Dosimetry Performance - Criteria for Testing. ANSI/HPS N13.11, Health Physics Society, McLean, Virginia. 
Health Physics Society (HPS). 1997. American National Standard - Criteria for Performing Multiple Dosimetry. HPS N13.41-1997, Health Physics Society, McLean, Virginia

Horowitz, Y. S. 1984. Thermoluminescence and Thermoluminescent Dosimetry. CRC Press, Inc., Boca Raton, Florida

International Atomic Energy Agency (IAEA). 1982. Dosimetry for Criticality Accidents, A Manual. Technical Report Series No. 211, IAEA, Vienna, Austria.

International Commission on Radiation Units and Measurements (ICRU). 1976. Conceptual Basis for the Determination of Dose Equivalent. ICRU Report 25, Bethesda, Maryland.

International Commission on Radiation Units and Measurements (ICRU). 1988. Determination of Dose Equivalents from External Radiation Sources - Part 2. ICRU Report 43, Bethesda, Maryland.

International Commission on Radiation Units and Measurements (ICRU). 1992. Measurement of Dose Equivalents from External Photon and Electron Radiations. ICRU Report 47, Bethesda, Maryland.

International Commission on Radiation Units and Measurements (ICRU). 1993. Quantities and Units in Radiation Protection Dosimetry. ICRU Report 51, Bethesda, Maryland.

International Commission on Radiation Units and Measurements (ICRU). 1998. Conversion Coefficients for use in Radiological Protection Against External Radiation. ICRU Report 57, Bethesda, Maryland.

International Commission on Radiological Protection (ICRP). 1973. Data for Protection against Ionizing Radiation from External Sources: Supplement to ICRP Publication 15. ICRP Publication 21. Pergamon Press, New York.

International Commission on Radiological Protection (ICRP). 1974. Report of the Task Group on Reference Man. ICRP Publication 23. Pergamon Press, New York.

International Commission on Radiological Protection (ICRP). 1977. Recommendations of the ICRP. ICRP Publication 26, New York, New York.

International Commission on Radiological Protection (ICRP). 1987. Data for Use in Protection Against External Radiation. ICRP Publication 51, New York, New York.

International Commission on Radiological Protection (ICRP). 1991. 1990 Recommendations of the International Commission on Radiological Protection. ICRP Publication 60, New York, New York.

International Commission on Radiological Protection (ICRP). 1996. Conversion Coefficients Use in Radiological Protection Against External Radiation. ICRP Publication 74, New York, New York.

International Organization for Standardization (ISO). 1989. ISO 8529-1989 Neutron reference radiations for calibrating neutron-measuring devices used for radiation protection purposes and for determining their response as a function of neutron energy. ISO 8529-1989, Geneva Switzerland.

International Organization for Standardization (ISO). 1998. ISO 8529-1998 Reference Neutron Radiations - Part 3: Calibration of area and personal dosimeters and determination of their response as a function of neutron energy and angle of incidence. ISO 8529-1998, Geneva Switzerland. 
Johnson, T. L. , and R. B. Luersen. 1980. "Fading of Unannealed LiF (TLD 600) for Thermal Neutrons and Gamma Rays” Health Physics Vol 38 pp 853-856.

Kocher, D. C. and K. F. Eckerman. 1987. "Electron Dose-Rate Conversion Factors for External Exposure of the Skin from Uniformly Deposited Activity on the Body Surface.” Health Physics 53 (2) 135-141.

McDonald, J. C., J. E. Tanner, R. D. Stewart, R. Michel, M. K. Murphy and R. J. Traub. 1995. "Effect of Phantom Size and Composition on Neutron Dosimeter Reading," Radiation Protection Dosimetry Vol 59, pp 263268 (1995)

McKeever, S. W. S., M. Moscovitch and P. D. Townsend. 1995. Thermoluminescence Dosimetry Materials: Properties and Uses, Nuclear Technology Publishing

McKinlay, A. F. 1981. Thermoluminescence Dosimetry. Adam Hilger, Ltd., Bristol, England.

Mettler, F. A. and G. Voelz 2001. "Evaluation of Neutron Exposure” in Medical Management of Radiation Accidents $2^{\text {nd }}$ Edition, (I. Gusev, A. Guskova, and F. Mettler, editors) CRC Press LLC. 2001.

Moscovitch, M. 1993. “Dose Algorithms for Personal Thermoluminescence Dosimetry” Radiation Protection Dosimetry Vol 47 pp 373-380 (1993)

Moscovitch, M. and J. E. Rotunda. 1996. Multi-Element Dosimetry System Using Neural Network. U.S. Patent Number 5,572,028, U.S. Patent Office

Moscovitch, M. 1999. "Personnel Dosimetry Using LiF:Mg,Cu,P”, Radiation Protection Dosimetry, Vol. 85, pp. 49-56.

National Council on Radiation Protection and Measurements (NCRP). 1971. Protection Against Neutron Radiation. NCRP Report No. 38, Bethesda, Maryland.

National Council on Radiation Protection and Measurements (NCRP). 1978 Instrumentation and Monitoring Methods for Radiation Protection, NCRP Report No. 57, Bethesda, Maryland

National Council on Radiation Protection and Measurements (NCRP). 1987 Ionizing Radiation Exposure of the Population of the United States, NCRP Report No. 93, Bethesda, Maryland

National Council on Radiation Protection and Measurements (NCRP). 1989. Limit for Exposure to "Hot Particles" on the Skin. NCRP Report No.106, Bethesda, Maryland.

National Council on Radiation Protection and Measurements (NCRP). 1992. Maintaining Radiation Protection Records. NCRP Report No. 114, Bethesda, Maryland.

National Council on Radiation Protection and Measurements (NCRP). 1993. Limitation of Exposure to Ionizing Radiation. NCRP Report No.116, Bethesda, Maryland.

National Council on Radiation Protection and Measurements (NCRP). 1995. Use of Personal Monitors to Estimate Effective Dose Equivalent and Effective Dose To Workers for External Exposure to Low-LET Radiation. NCRP Report No. 122, Bethesda, Maryland.

National Council on Radiation Protection and Measurements (NCRP). 1999. Biological Effects and Exposure 
Limits for “Hot Particles”. NCRP Report No. 130, Bethesda, Maryland.

Oberhofer M. and A. Scharmann (editors). 1979. Applied Thermoluminescent Dosimetry. p 208, Adam Hilger Ltd, Bristol, England.

Plato, P., and Miklos, J., 1985. "Production of Element Correction Factors for Thermoluminescent Dosimeters" Health Physics Vol 49, No. 5 (November), pp 873-881, 1985

Piesch, E. Burgkhardt, B. and Venkataraman, G. 1982 Study of the Phantom Distance Effect of Albedo Neutron Dosimeters, Radiation Protection Dosimetry Vol. 3 No. 1/2 1982 pp. 39-45

Piesch, E. and Burgkhardt, B. 1985 Albedo Neutron Dosimetry, Radiation Protection Dosimetry Vol. 10 No. 1-4 pp. 175-188 (1985)

Rathbone, B. A., A. W. Endres, E. J. Antonio. 1994. "Evaluation of New and Conventional Thermoluminescent Phosphors for Environmental Monitoring Using Automated Thermoluminescent Dosimeter Readers,”

Proceedings of Fourth Conference on Radiation Protection and Dosimetry, ORNL/TM-12817.

Rathbone, B. A., J. C. McDonald, and R. J. Traub. 2002. "Current Challenges in Personnel Dosimetry at the US DOE Hanford Site” Radiation Protection Dosimetry 101(1-4):153-166. (2002)

Reginatto, M. and P. Goldhagen. 1998. MAXED, A Computer Code for the Deconvolution of Multisphere Neutron Spectrometer Data Using the Maximum Entropy Method. EML-595.

Environmental Measurements Laboratory, New York, NY.

Roberson P. L. and R. D. Carlson. 1992. Determining the Lower Limit of Detection for Personnel Dosimetry Systems, Health Physics, Vol 49, No. 1 (January), pp 2-9, 1992

Robertson, M.E.A., and W.B. Gilboy. 1971. Studies of the Thermoluminescence of Lithium Fluoride Doped with Various Activators, Proc. Third Int. Conf. Luminescence Dosimetry, Rïso-Rep, 249, Vol. 1, Danish AEC, Rïso, Roskilde, Denmark.

Rohloff, F. and Heinzelmann, M. 1986. "Calculation of Dose Rates for Skin Contamination by Beta Radiation.” Radiation Protection Dosimetry 14(4): 279-287.

Scherpelz, R. I., J. J. Fix, and B. A. Rathbone, 2000. Validation of Hanford Personnel and Extremity Dosimeters in Plutonium Environments, PNNL-13136, Pacific Northwest National Laboratory, Richland, Washington

Scherpelz, R. I., and M. M. Conrady. 2008. Upgrade of the PNNL TEPC and Multisphere Spectrometer . PNNL-17809, Pacific Northwest National Laboratory, Richland, WA.

Schwartz, R. B., and C. M. Eisenhauer. 1982. Procedures for Calibrating Neutron Personnel Dosimeters, NBS Special Publication 633, U.S. Department of Commerce/National Bureau of Standards, Washington, DC.

Seagraves, D. T., B. R. Madrid, C. D. Roybal, R. L. Markham, and T. D. McLean, 2008 Characterization of the Hanford 8816 personnel dosimeter at the Los Alamos National Laboratory Central Health Physics Calibration Facility (TA-36-214), LA-UR-08-06504, Los Alamos National Laboratory, Los Alamos, NM. September 2008.

Sherbini, S, J Decicco, A Turner-Gray, and R Struckmeyer. 2008. "Verification of the VARSKIN Beta Skin Dose Calculation Computer Code” Health Physics, Volume 94, Number 6, June 2008. 
Storm, E., P. L. Buslee, A. W. Blackstock, G. J. Littlejohn, J. R. Cortez, R. V. Fultyn, and J. N. P. Lawrence. 1981. “The Los Alamos Thermoluminescence Dosimeter Badge.” Radiation Protection Dosimetry 1 (3): 209-219.

Takahashi, F., A. Endo and Y. Yamaguchi. 2003. "Dose Assessment from Activated Sodium Within a Body in Criticality Accidents." Radiation Protection Dosimetry 106(3):197-206.

Tawil, R.A. 1996. “Thermoluminescent Dosimetry” Applications of New Technology: External Dosimetry, Health Physics Society, 1996

Tawil, R. A., K. J. Velbeck, J. E. Rotunda, C. Flory, and M. Moscovitch. 1993. "A System for Environmental Monitoring." Radiation Protection Dosimetry 47(1/4):317-321.

U.S. Department of Energy (DOE). 1986a. Department of Energy Standard for the Performance Testing of Personnel Dosimetry Systems. DOE/EH-0027, Washington D.C.

U.S. Department of Energy (DOE). 1986b. Handbook for the Department of Energy Accreditation Program for Personnel Dosimetry Systems. DOE/EH-0026, Washington D.C.

U.S. Department of Energy (DOE). 1993. Occupational Radiation Protection.. Federal Register. Title 10 Part 835. U.S., Code of Federal Regulations, Vol. 58, No. 238, 65458-65512.

U.S. Department of Energy (DOE) 1995. Department of Energy Laboratory Accreditation Program for Personnel Dosimetry Systems. DOE-STD-1095-95

U.S. Department of Energy (DOE) 1998a. The Department of Energy Laboratory Accreditation Program Administration. DOE-STD-1111-98

U.S. Department of Energy (DOE) 1998b. Guide of Good Practices for Occupational Radiological Protection in Plutonium Facilities. DOE-STD-1128-98

U.S. Department of Energy (DOE). 1998c. Occupational Radiation Protection.. Federal Register. Title 10 Part 835. U.S., Code of Federal Regulations, Vol. 63, No. 213, 59662-59689.

U.S. Department of Energy (DOE). 1999. DOE Standard - Radiological Control. DOE-STD-1098-99, Washington, D.C.

U.S. Department of Energy (DOE) — Richland Operations Office. 2001 Hanford Radiological Health and Safety Document. DOE/RL-2002-12, Richland, Washington.

U.S. Department of Energy (DOE), 2003. Quality Assurance Requirements. U.S. Code of Federal Regulations, Title 10 Part 830 - Subpart A, .

U.S. Department of Energy (DOE), 2004 DOE O 231.1A, Change 1, Environment, Safety and Health Reporting, U.S. Department of Energy. Washington, D.C. 2004

U.S. Department of Energy (DOE). 2005a. Quality Assurance. DOE O 414.1C, DOE Orders and Directives.

U.S. Department of Energy (DOE). 2005b. DOELAP Information Advisory - Configuration of DOELAP Dosimeters, Office of Environment Safety and Health, U.S. Department of Energy, December 5, 2005. 
U.S. Department of Energy (DOE). 2006. DOELAP Information Notice - Configuration of DOELAP Dosimeters, Office of Environment Safety and Health, U.S. Department of Energy, July 13, 2006.

U.S. Department of Energy (DOE). 2007a. Occupational Radiation Protection Federal Register, June 8, 2007. Title 10 Part 835. U.S., Code of Federal Regulations, Vol. 72, No. 110, 31904-31941.

U.S. Department of Energy (DOE). 2007b. DOE M 231.1-1A, Change 2, 6-12-2007, Environment, Safety and Health Reporting Manual, U.S. Department of Energy. Washington, D.C.

U.S. Department of Energy (DOE). 2008a. Radiation Protection Programs Guide. DOE G 441.1-1C, May 19, 2008, Washington, D.C.

U.S. Department of Energy (DOE). 2008b. Radiological Control DOE-STD-1098-2008, October, 2008, Washington, D.C.

U.S. Department of Energy (DOE). 2009. Technical Position Clarifying Dose Assessment and Recording. RCTP 2009-01, DOE Office of Worker Safety and Health Policy, March 6, 2009, Washington, D.C.

U.S. Nuclear Regulatory Commission (NRC). 1977. Performance, Testing, and Procedural Specifications for Thermoluminescence Dosimetry: Environmental Applications. Regulatory Guide 4.13, Washington, D.C.

Vasilik, D. G., and R. W. Martin. 1981a. The Los Alamos Personnel and Area Criticality Dosimeter Systems. LA-8848-MS, Los Alamos Scientific Laboratory, Los Alamos, New Mexico.

Vasilik, D. G., and R. W. Martin. 1981b. Nuclear Accident Dosimetry: Los Alamos Measurements at the Seventeenth Nuclear Accident Dosimetry Intercomparison Study at the Oak ridge National Laboratory DOSAR Facility, August 1980. LA-8799-MS, Los Alamos Scientific Laboratory, Los Alamos, New Mexico.

Wilson, R. H., J. J. Fix, W. V. Baumgartner, and L. L. Nichols. 1990. Description and Evaluation of the Hanford Personnel Dosimeter Program From 1944 Through 1989. PNL-7447, Pacific Northwest Laboratory, Richland, Washington.

Wilson, R. H. 1962. A Method for Immediate Detection of High Level Neutron Exposure by Measurement of Sodium-24 in Humans. HW-73891 Rev. General Electric, Richland, Washington.

Wilson, R. H. 1987. Historical Review of Personnel Dosimetry Development and its Use in Radiation Protection Programs at Hanford. PNL-6125, Pacific Northwest Laboratory, Richland, Washington.

Xu, X. G. 2005. "Effective Dose Equivalent and Effective Dose for Hot Particles on the Skin” Health Physics, Volume 89, Number 1, pp 53-70, July 2005.

Xu, X. G., H. Su, and S. Bushart 2006. “The EPRI EDE Calculator - A Software Package for Assessing Effective Dose Equivalent from Hot Particles on the Skin” Health Physics, Volume 91, Number 4, pp 373-378, October 2006. 